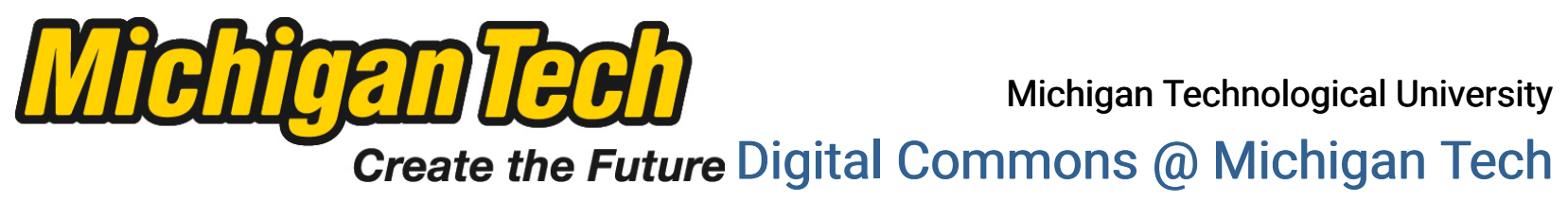

Dissertations, Master's Theses and Master's Reports - Open

2015

\title{
MODELING AND SIMULATION OF MICROSTRUCTURES, MECHANISMS, AND DIFFRACTION EFFECTS IN ENERGY MATERIALS: FERROELECTRICS AND LITHIUM ION BATTERY CATHODE MATERIALS
}

Jie Zhou

Michigan Technological University

Follow this and additional works at: https://digitalcommons.mtu.edu/etds

Part of the Materials Science and Engineering Commons, and the Physics Commons Copyright 2015 Jie Zhou

Recommended Citation

Zhou, Jie, "MODELING AND SIMULATION OF MICROSTRUCTURES, MECHANISMS, AND DIFFRACTION EFFECTS IN ENERGY MATERIALS: FERROELECTRICS AND LITHIUM ION BATTERY CATHODE MATERIALS", Dissertation, Michigan Technological University, 2015.

https://doi.org/10.37099/mtu.dc.etds/983

Follow this and additional works at: https://digitalcommons.mtu.edu/etds

Part of the Materials Science and Engineering Commons, and the Physics Commons 


\title{
MODELING AND SIMULATION OF MICROSTRUCTURES, MECHANISMS, AND DIFFRACTION EFFECTS IN ENERGY MATERIALS: FERROELECTRICS AND LITHIUM ION BATTERY CATHODE MATERIALS
}

\author{
By \\ Jie Zhou \\ A DISSERTATION \\ Submitted in partial fulfillment of the requirements for the degree of \\ DOCTOR OF PHILOSOPHY \\ In Materials Science and Engineering \\ MICHIGAN TECHNOLOGICAL UNIVERSITY \\ 2015 \\ (C) 2015 Jie Zhou
}


This dissertation has been approved in partial fulfillment of the requirements for the Degree of DOCTOR OF PHILOSOPHY in Materials Science and Engineering.

Department of Materials Science and Engineering.

Dissertation Advisor: $\quad Y u U$. Wang

Committee Member: Jaroslaw W. Drelich

Committee Member: $\quad$ Stephen A. Hackney

Committee Member: Yang Ren

Committee Member: $\quad$ Yongmei M. Jin

Department Chair: $\quad$ Stephen L. Kampe 


\section{Contents}

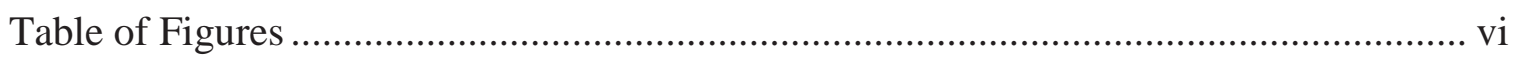

Preface

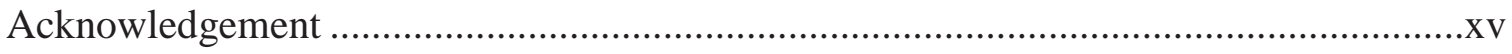

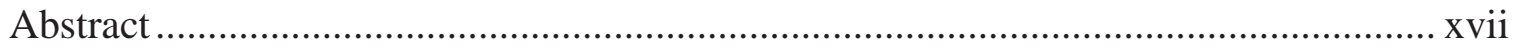

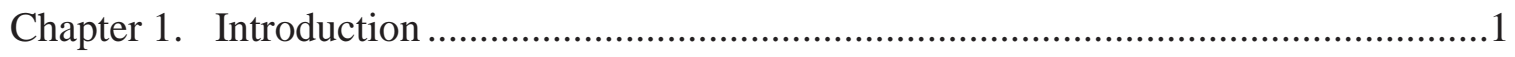

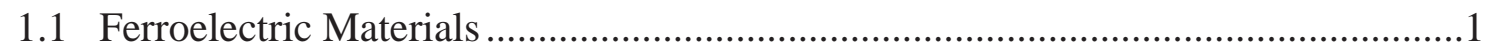

1.1.1 Domain Mechanisms for Dielectric and Piezoelectric Behaviors in

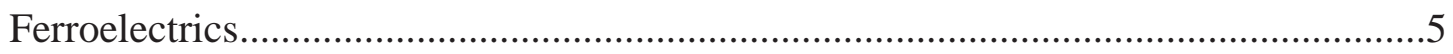

1.1.2 Domain-Level Modeling and Simulation Method: Phase Field Model.............10

1.1.3 Texture Development and Computational Diffraction .......................................13

1.2 Lithium Ion Battery Cathode Materials ……………………….............................14

1.2.1 Capacity Fade Phenomenon of the Cathode Materials .......................................16

1.2.2 Nanoscale Structural Defects: Stacking Fault and Ordering .............................18

1.2.3 Nanoscale Computational Diffraction Method: DIFFaX ………………….......21

Chapter 2. Computational Methodology ..................................................................25

2.1 Phase Field Model of Ferroelectric Materials........................................................25

2.2 Phase Field Model of Templated Grain Growth....................................................27

2.3 Phase Field Model of Two-Phase Composites ………...............................................30

2.4 Computational Diffraction of Textured Polycrystals.............................................31

2.5 Computational Diffraction of Nanoscale Intergrowth of Layered Structures with

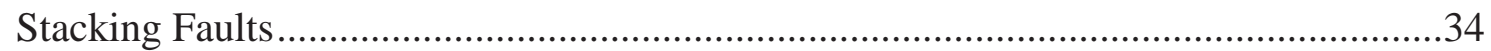

Chapter 3. Domain Engineering for Ferroelectric Single Crystal.....................................36

3.1 Landau-Type Bulk Free Energy Landscape …………….......................................39

3.2 Phase Field Simulation of Electrical Field Induced Domain Structure Design ......40

3.3 Phase Field Simulation of Domain Size Control ...................................................45

3.4 Theoretical Study and Numerical Simulation for Correlated Nucleation................49

3.4.1 The Limitations of Classical Isolated Nucleation Theory in Ferroelectric

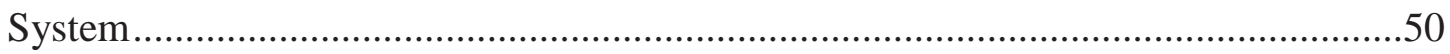


3.4.2 Correlated Nucleation Process and Domain Formation .60

3.4.3 Induced Heterogeneous Nucleation to Design Stripe Domain ........................72

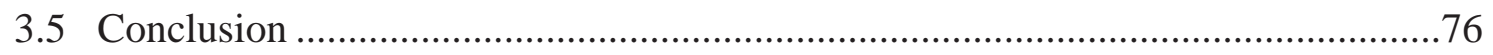

Chapter 4. Grain Texturing by Templated Grain Growth.............................................77

4.1 Phase Field Simulation of Templated Grain Growth........................................79

4.2 X-ray Diffraction Calculation to Characterize Texture Development during Sintering..... .82

4.3 Simulation Validation by Comparison with Experiment....................................8

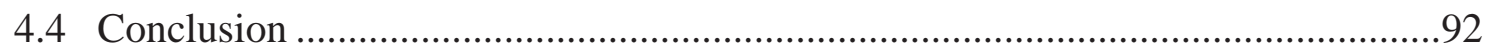

Chapter 5. Effects of Crystallographic Texture and Grain Shape Anisotropy on Piezoelectric Properties of Ferroelectric Polycrystalline Ceramics ..................................94

5.1 Grain Shape Effect on Ferroelectric Properties ..................................................96

5.2 Grain Texture Effect on Ferroelectric Properties...............................................104

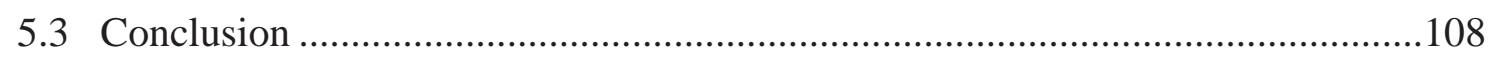

Chapter 6. Phase Field Modeling of Textured Ferroelectric Polycrystals: Dielectric and Piezoelectric Properties of Template-Matrix Composites ............................................110

6.1 Texture Effects on Ferroelectric Properties in Two-Phase Composites ...............113

6.2 Competing Composites Effects on Dielectric and Piezoelectric Properties .........120

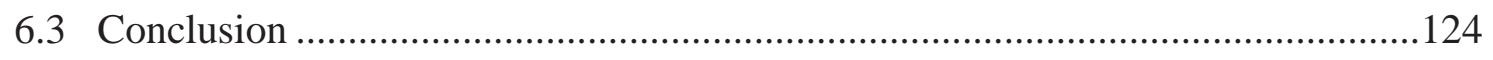

Chapter 7. Diffraction Analysis of Lithium Ion Battery Cathode Materials ................126

7.1 Analysis of $x \mathrm{LiCoO}_{2}-(1-x) \mathrm{Li}_{2} \mathrm{MnO}_{3}$ Lithium Ion Battery Cathode Materials: A

Hierarchical Structural Refinement Approach......................................................... 127

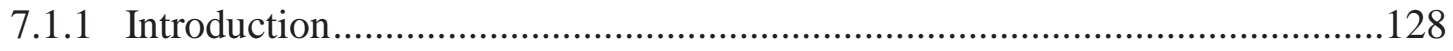

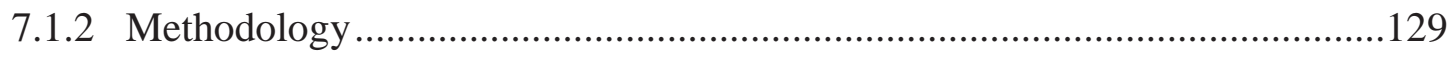

7.1.3 Distributions of Stacking Faults as Function of Composition .......................134

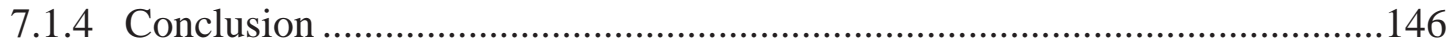

7.2 Analysis of Toda HE5050 Lithium Ion Battery Cathode Materials: Solid Solution,

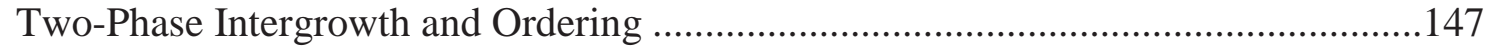

7.2.1 Structural Nature of Solid Solution versus Two-Phase Intergrowth ..............150

7.2.2 Hierarchical Structural Refinement Approach Applied to HE5050 ................159

7.3 Faulting in Oxygen Framework and Vacancies ...............................................161 


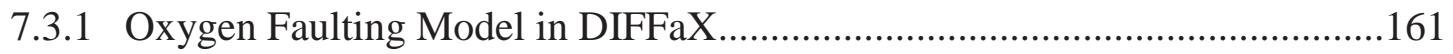

7.3.2 Extraordinary X-ray Diffraction Phenomenon due to Oxygen Faulting ........166

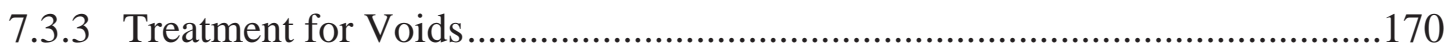

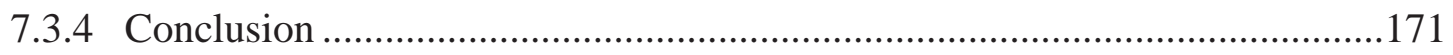

Chapter 8. Summary and Prospective Research .....................................................173

8.1 Summary of Results from Computational Studies............................................173

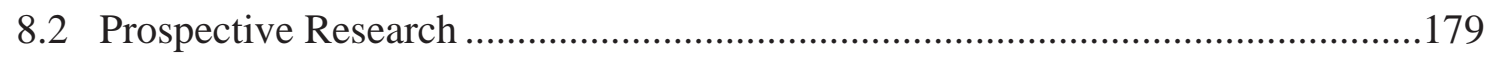

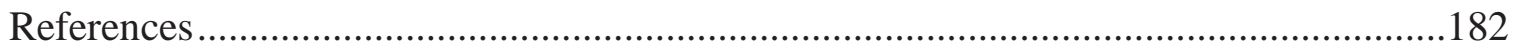

Appendix A: Energy Barrier to Ferroelectric Nucleation Due to Electrostatic and

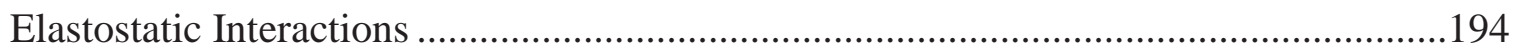

Appendix B: DIFFaX Resolution and Broadening Parameters ..................................200

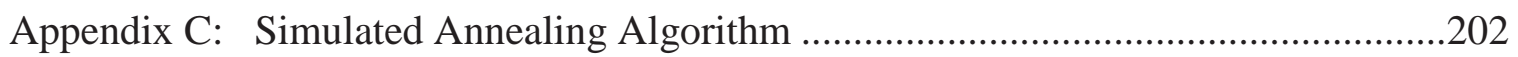

Appendix D: Copyright Permission of Texts and Figures in Chapter 3 and Copyright

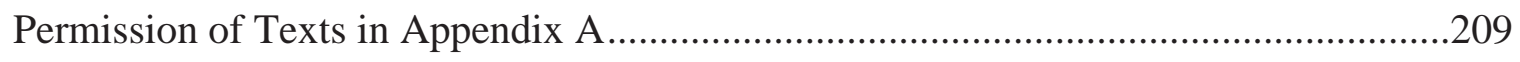

Appendix E: Copyright Permission of Texts and Figures in Chapter 4 ......................213

Appendix F: Copyright Permission of Figures in Chapter 1 ...................................215

Appendix G: Permission for Use of Figure 4.10 in Chapter 4 ...................................217 


\section{Table of Figures}

Figure 1.1 Phase diagram for (a) PZT (b) PMN-PT (c) PZN-PT ...................................3

Figure 1.2 Simulated Grain Structures for (a) equiaxed polycrystal (b) polycrystal with template seed (c) polycrystal with elongated grain shape. Stereographic projections of (010) plane grain orientation distributions for (d-1) untextured $\left(90^{\circ}\right)(\mathrm{d}-2)$ intermediately

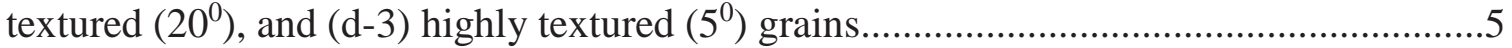

Figure 1.3 Schematic lattice geometries for common ferroelectric phases and

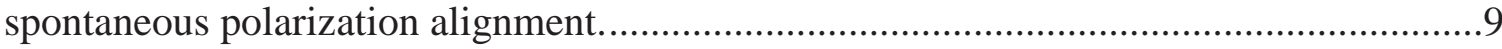

Figure 1.4 Demonstration for the working principle in lithium ion battery.................16 Figure 1.5 (a) Visualized $\mathrm{Li}_{2} \mathrm{MnO}_{3}$ layered structure. (b) Visualized $\mathrm{LiMn}_{2} \mathrm{O}_{4}$ spinel layered structure. .18

Figure 1.6 Stacking faults for layered $\mathrm{Li}_{2} \mathrm{MnO}_{3}$ structure with translation of stacking vectors.

Figure 2.1 Layered structures (a) perfect stacking (b) stacking faults. .35

Figure 3.1 Topologies of bulk free energy iso-surface for different phases in PZT. (a) Cubic phase. (b) Tetragonal phase. (c) Rhombohedral phase.

Figure 3.2 Computer simulation of domain evolution and twin microstructure formation in single crystals under external electric field applied along non-polar axes during ferroelectric phase transition.

Figure 3.3 (a) Simulated herringbone microstructure under [100] electric field. (b) Simulation of correlated ferroelectric nucleation.

Figure 3.4 Supplementary possible final domain structures when applied with [100] electric field in rhombohedral phase.

Figure 3.5 Computer simulation of dependence of domain wall density in engineered domain configuration on non-polar electric field magnitude. .48

Figure 3.6 Computer simulation of polarization evolution and pattern formation during early stage of ferroelectric phase transformation. An important feature of early-stage 
transformation is that vortex structures remain isotropic during coarsening and polarization growth.

Figure 3.7 Enlarged (10 $\times$ ) portion of Fig. 3.6(a) to show the details of polarization vortex structures formed by correlated nucleation during early-stage ferroelectric phase transformation.

Figure 3.8 Computer simulation of polarization evolution and pattern formation during intermediate stage of ferroelectric phase transformation. An important feature of intermediate-stage transformation is that isotropic vortex structures gradually transform into charge-accommodating bridging domain structures with preferred polarization orientations .66

Figure 3.9 Computer simulation of polarization evolution and pattern formation during late stage of ferroelectric phase transformation. An important feature of late-stage transformation is that charge-accommodating bridging domain structures develop into fully self-accommodating twinned domain structures with head-to-tail polarization patterns that simultaneous minimize both electrostatic and elastostatic energies

Figure 3.10 Computer simulation of wavelength (wavenumber) dependence of polarization evolution during early stage of ferroelectric phase transformation. Spectral distributions are calculated by fast Fourier transform of the polarization fields in Fig. 3.6(a)-(d).

Figure 3.11 Computer simulation of polarization evolution and pattern formation during early stage of ferroelectric phase transformation via local field-induced heterogeneous nucleation.

Figure 3.12 Computer simulation of polarization evolution and pattern formation subsequent to local field-induced heterogeneous nucleation. .75

Figure 4.1 Simulated process of grain growth without template seed. .80

Figure 4.2 Simulated process of templated grain growth with one template seed per simulation box (volume fraction $0.63 \%$ ).

Figure 4.3 Simulated process of templated grain growth with three template seeds per simulation box (volume fraction $2.37 \%$ ). 
Figure 4.4 Calculated integrated peak intensities of untextured $\mathrm{SrTiO}_{3}$ polycrystals and comparison with Powder Diffraction File (PDF).

Figure 4.5 Calculated evolutions of X-ray diffraction peak intensities during grain growth processes corresponding to the evolving grain structures in Figs. 4.1, 4.2 and 4.3, respectively. Template volume fraction: (a) $0 \%$, (b) $0.63 \%$, and (c) $2.37 \%$. .84

Figure 4.6 Evolution of Lotgering factor during grain growth processes corresponding to the evolving grain structures in Figs. 4.1, 4.2 and 4.3, respectively, as calculated from the X-ray diffraction peak intensities in Fig. 4.5. .85

Figure 4.7 Effects of template volume fraction and template seed number on Lotgering factor in fully sintered polycrystals.

Figure 4.8 Effects of template volume fraction and template seed number on Lotgering factor in intermediately sintered polycrystals.

Figure 4.9 Simulated evolution of Lotgering factor during template grain growth processes of same template volume fraction $(2.1 \%)$ but different template seed numbers (thus different template seed sizes). .88

Figure 4.10 SEM images of fractured cross-sections of PMN-32.5\%PT polycrystalline ceramics with $3 \%$ volume fraction of BT templates sintered at $900^{\circ} \mathrm{C}$ for different time $t$ : (a) $1 \mathrm{~min}$, (b) $3 \mathrm{~min}$, (c) $5 \mathrm{~min}$, (d) $10 \mathrm{~min}$, (e) $20 \mathrm{~min}$, and (f) $40 \mathrm{~min}$. (Supplied by Y. Yan and S. Priya at Virginia Tech.). .90

Figure 4.11 Experimental X-ray diffraction patterns of PMN-32.5\%PT polycrystalline ceramics with $3 \%$ volume fraction of BT templates sintered at $900^{\circ} \mathrm{C}$ for different time. (Supplied by Y. Yan and S. Priya at Virginia Tech.) .91

Figure 4.12 Comparison of experimental and computational evolutions of Lotgering factor with sintering time.

Figure 5.1 Polycrystalline grain structures of different grain shape anisotropies, namely, (a) equiaxed grains of average shape aspect ratio 1:1, and elongated grains of average shape aspect ratio (b) $2: 1$ and (c) 4:1.

Figure 5.2 Stereographic projections of (010) plane orientation distributions for (a) untextured $\left(\theta=90^{\circ}\right)$, (b) intermediately textured $\left(\theta=20^{\circ}\right)$, and (c) highly textured $\left(\theta=5^{\circ}\right)$ grains. 
Figure 5.3 (a) Equiaxed (1:1) grain structure shown in Fig. 5.1. (a') Corresponding to mirror operation about the diagonal of (a) itself. (b) Elongated (2:1) grain structure shown in Fig. 5.1. (b') Corresponding to mirror operation about the diagonal of (b) itself. (c) Elongated (4:1) grain structure shown in Fig. 5.1. (c') Corresponding to mirror operation about the diagonal of (c) itself.

Figure 5.4 Simulated $P-E$ hysteresis loop measured along [100] based on the (a) Equiaxed grain structure and its mirror. (b) Elongated (2:1) grain structure and its mirror. (c) Elongated (4:1) grain structure and its mirror. Simulated $\varepsilon$-E hysteresis loop measured along [100] based on the grain structure (d) Equiaxed grain structure and its mirror. (e) Elongated (2:1) grain structure and its mirror. (f) Elongated (4:1) grain structure and its mirror. (g), (h) and (i) are obtained from (d), (e) and (f) respectively by shifting the two $\varepsilon$ $E$ hysteresis loops on each graph to a common reference strain position. 100

Figure 5.5 Selected typical domain patterns for grain ratio (4:1) (a) After poling $(\mathrm{E}=0 \mathrm{kV} / \mathrm{cm})$ for normal grain structure. (b) After poling $(\mathrm{E}=0 \mathrm{kV} / \mathrm{cm})$ for mirror grain structure. (c) Around switching point for normal grain structure. (d) Around switching point for mirror grain structure. .101

Figure 5.6 Arrangement of hypothetical grains with infinite shape aspect ratio and related $E-P$ loops and $\varepsilon-E$ loops. .102

Figure 5.7 Simulated $P-E$ hysteresis loop for untextured polycrystals of different grain shape aspect ratios $(1: 1,2: 1,4: 1)$ as shown in Fig. 5.1, measured along (a) [100] (horizontal) and (b) [010] (vertical) directions. .103

Figure 5.8 (a) Simulated $P-E$ hysteresis loops for different values of texture for polycrystals of grain shape aspect ratios (1:1). (b) Simulated $\varepsilon$-E hysteresis loops for different values of texture for polycrystals of grain shape aspect ratios $(1: 1) \ldots \ldots \ldots \ldots \ldots . . .106$ Figure 5.9 Extracted values from Fig. 5.8 for ferroelectric properties (a) Saturation polarization $\left(P_{S}\right)($ b) Coercivity (c) Dielectric constant (d) Piezoelectric coefficient vary with distribution angle.

Figure 5.10 Phase field calculated piezoelectric coefficient $d_{33}$ with distribution angle, which is the same data set as shown in Fig. 5.9(d). Blue curve and points are obtained from 
Eq. (5.1) with $\bar{Q}_{11}=Q_{11}$ which determines upper bound for intrinsic values of $\mathrm{d}_{33}$. Green curve and points are obtained from Eq. (5.1) with $\bar{Q}_{11}=<Q_{11}>$ which determines lower bound for intrinsic values of $\mathrm{d}_{33}$ .108

Figure 6.1 (a) Grain structure and (b) phase morphology of polycrystalline ferroelectric composite textured by templated grain growth. .113

Figure 6.2 Simulated domain patterns quenching through Curie temperature: (a) two phase composite polycrystalline domains. (b) Zoom in portion of (a) close to template surface. (c) Pure PZT phase counterpart with the same grain structure and orientation as two phase composites. (d) Zoom in portion of (c) close to template surface. Solid black lines represent grain structure similar to Fig. 6.1 (a). .114

Figure $\quad 6.3$ (a) E-P Loop (b) $\varepsilon$-E Loop at different reduced simulation time step. .......116 Figure 6.4 (a) Remnance (b) Coercivity (c) Dielectric Constant ( $\left.\varepsilon_{33}\right)$ (d) Piezoelectric Coefficient $\left(\mathrm{d}_{33}\right)$ with time evolution based on grain structure at bottom of Fig. 6.3 ....117 Figure 6.5 E-P curves and snapshots of domain patterns close to template seed. (a)-(f) on the right side are selected area of domain patterns adjacent to phase boundary under different electric field strength. The rightmost column represents domain patterns for pure PZT with the same grain structure and at the same loading stage as composites for comparison purpose. 118

Figure 6.6 (a) E-P curves of pure PZT, pure BTO, and two phase composites with template volume fraction $0.63 \%$. (b) E-P curves of pure PZT, pure BTO, and two phase composites with template volume fraction $2.37 \%$. The insets on the corner of (a) and (b) show grain structures based on which E-P curves are calculated.

Figure 6.7 (a) Remnance (b) Coercivity (c) Dielectric Constant ( $\left.\varepsilon_{33}\right)$ (d) Piezoelectric Coefficient $\left(\mathrm{d}_{33}\right)$ via seed number.

Figure 6.8 (a) Lotgering Factor (b) Remnance (c) Coercivity (d) Dielectric Constant ( $\left.\varepsilon_{33}\right)$ (e) Piezoelectric Coefficient $\left(\mathrm{d}_{33}\right)$ via seed volume fraction.

Figure 6.9 Piezoelectric Coefficient $\left(\mathrm{d}_{33}\right)$ via seed volume fraction (red curve). The upper bound (blue curve) obtained from Eq. (5.1) with $\bar{Q}_{11}=Q_{11}$. The lower bound (green curve) obtained from Eq. (5.1) with $\bar{Q}_{11}=<Q_{11}>$ 
Figure 7.1 Schematic illustration of a hierarchical refinement strategy for structural analysis of layered two-phase intergrowth materials with stacking faults.

Figure 7.2 Illustration of two-phase intergrowth model of $\mathrm{LiCoO}_{2}-\mathrm{Li}_{2} \mathrm{MnO}_{3}$ with stacking faults, as visualized using VESTA.

Figure 7.3 Comparison of fitting residual error $R_{P}$ among solid solution model, twophase intergrowth model with homogeneous and spatially heterogeneous distributions of stacking faults.

Figure 7.4 Comparison of two-phase intergrowth model with stacking faults and solid solution model for pure $\mathrm{Li}_{2} \mathrm{MnO}_{3}(x=0)$. (a) Powder diffraction intensity profiles of experiment, solid solution model, and two-phase intergrowth model with heterogeneous distributions of stacking faults. (b) Fitting residual error $R_{P}$ of solid solution model and two-phase intergrowth model with different homogeneous stacking fault probabilities. Best fitted spatially heterogeneous distributions of stacking faults are also shown in (b). ....136 Figure 7.5 Comparison of two-phase intergrowth model with stacking faults and solid solution model for $10 \% \mathrm{LiCoO}_{2}-90 \% \mathrm{Li}_{2} \mathrm{MnO}_{3}(x=0.1)$.

Figure 7.6 Comparison of two-phase intergrowth model with stacking faults and solid solution model for $30 \% \mathrm{LiCoO}_{2}-70 \% \mathrm{Li}_{2} \mathrm{MnO}_{3}(x=0.3)$.

Figure 7.7 Comparison of two-phase intergrowth model with stacking faults and solid solution model for $50 \% \mathrm{LiCoO}_{2}-50 \% \mathrm{Li}_{2} \mathrm{MnO}_{3}(x=0.5)$.

Figure 7.8 Comparison of two-phase intergrowth model with stacking faults and solid solution model for $70 \% \mathrm{LiCoO}_{2}-30 \% \mathrm{Li}_{2} \mathrm{MnO}_{3}(x=0.7)$.

Figure 7.9 Comparison of two-phase intergrowth model with stacking faults and solid solution model for $90 \% \mathrm{LiCoO}_{2}-10 \% \mathrm{Li}_{2} \mathrm{MnO}_{3}(x=0.9)$.

Figure 7.10 Powder diffraction intensity profiles of experiment, solid solution model, and two-phase intergrowth model for pure $\mathrm{LiCoO}_{2}(x=1)$.

Figure 7.11 Illustration of different structures of $\mathrm{Li}_{2} \mathrm{MnO}_{3}$ produced by (a) V1 stacking, (b) V2 stacking, and (c) V2V3 stacking, as visualized using VESTA. .144 
Figure 7.12 Refinement of two-phase intergrowth model with stacking faults and different structures of $\mathrm{Li}_{2} \mathrm{MnO}_{3}$ phase produced respectively by $\mathrm{V} 2$ stacking and $\mathrm{V} 2 \mathrm{~V} 3$ stacking for $50 \% \mathrm{LiCoO}_{2}-50 \% \mathrm{Li}_{2} \mathrm{MnO}_{3}(x=0.5)$

Figure 7.13 DIFFaX implementation of unit cells, stacking sequence, stacking fault, and two-phase intergrowth, as visualized using VESTA.

Figure 7.14 DIFFaX simulation of X-ray powder diffraction of disordered solid solution $\mathrm{Li}_{1.2} \mathrm{Ni}_{0.15} \mathrm{Co}_{0.1} \mathrm{Mn}_{0.55} \mathrm{O}_{2}$ with different percentages of stacking faults. .152

Figure 7.15 DIFFaX simulation of X-ray powder diffraction of ordered solid solution $\mathrm{Li}_{1.2} \mathrm{Ni}_{0.15} \mathrm{Co}_{0.1} \mathrm{Mn}_{0.55} \mathrm{O}_{2}$ with different percentages of stacking faults.

Figure 7.16 DIFFaX simulation of X-ray powder diffraction of two-phase intergrowth of $0.5 \mathrm{Li}_{2} \mathrm{MnO}_{3}-0.5 \mathrm{Li}\left(\mathrm{Ni}_{3 / 8} \mathrm{Co}_{1 / 4} \mathrm{Mn}_{3 / 8}\right) \mathrm{O}_{2}$ with different percentages of stacking faults...153 Figure 7.17 Distinction of different structure models (disordered solid solution, ordered solid solution, two-phase intergrowth) and determination of composition $x$ of $x \mathrm{Li}_{2} \mathrm{MnO}_{3}$ $(1-x) \mathrm{Li}\left(\mathrm{Ni}_{3 / 8} \mathrm{Co}_{1 / 4} \mathrm{Mn}_{3 / 8}\right) \mathrm{O}_{2}$ via quantitative comparison of $\mathrm{DIFFaX}$ simulations with experimental data. .155

Figure 7.18 DIFFaX simulation of X-ray powder diffraction of $x \mathrm{Li}\left(\mathrm{Li}_{1 / 3} \mathrm{Mn}_{2 / 3}\right) \mathrm{O}_{2}-(1-$ $x) \mathrm{Li}\left(\mathrm{Ni}_{3 / 8} \mathrm{Co}_{1 / 4} \mathrm{Mn}_{3 / 8}\right) \mathrm{O}_{2}$ two-phase intergrowth with $20 \%$ stacking faults and different composition $x$. 156

Figure 7.19 Determination of stacking fault percentage of $0.5 \mathrm{Li}_{2} \mathrm{MnO}_{3}$ $0.5 \mathrm{Li}\left(\mathrm{Ni}_{3 / 8} \mathrm{Co}_{1 / 4} \mathrm{Mn}_{3 / 8}\right) \mathrm{O}_{2}$ (Toda HE5050) via quantitative comparison of DIFFaX simulations with experimental data. 156

Figure 7.20 DIFFaX simulation of X-ray powder diffraction of two-phase intergrowth of $0.5 \mathrm{Li}_{2} \mathrm{MnO}_{3}-0.5 \mathrm{Li}\left(\mathrm{Ni}_{3 / 8} \mathrm{Co}_{1 / 4} \mathrm{Mn}_{3 / 8}\right) \mathrm{O}_{2}$ with $20 \%$ and $25 \%$ stacking faults. .158

Figure 7.21 DIFFaX simulation of X-ray powder diffraction based on three different structure models (two-phase intergrowth, ordered solid solution, disordered solid solution) of $\mathrm{Li}_{1.2} \mathrm{Ni}_{0.15} \mathrm{Co}_{0.1} \mathrm{Mn}_{0.55} \mathrm{O}_{2}$ with $20 \%$ stacking faults

Figure 7.22 Comparison of two-phase intergrowth model with stacking faults and solid solution model for $50 \% \mathrm{Li}_{2} \mathrm{MnO}_{3}-50 \% \mathrm{Li}\left(\mathrm{Ni}_{3 / 8} \mathrm{Co}_{1 / 4} \mathrm{Mn}_{3 / 8}\right) \mathrm{O}_{2}$. (a) Powder diffraction intensity profiles of experiment, solid solution model, and two-phase intergrowth model with heterogeneous distributions of stacking faults. (b) Fitting residual error $R_{P}$ of solid xii 
solution model and two-phase intergrowth model with different homogeneous stacking fault probabilities. .161

Figure 7.23 ABC non-faulting stacking for oxygen layers.

Figure 7.24 Possible stacking vectors are designated with colorful dotted arrows corresponding to different stacking translations in monoclinic notation, which keep oxygen framework (ABC stacking) unchanged.

Figure 7.25 Oxygen layer faulting. 165

Figure 7.26 Projective views along monoclinic b axis (a) unit cell for perfect stacking with $[001]_{\text {mon }}$ stacking vector. (b) unit cell constructed for revealing oxygen layer faulting structure with possible stacking vectors $[1 / 3,0,1]_{\mathrm{mon}},[-1 / 6,-1 / 6,1]_{\mathrm{mon}}$, and $[-1 / 6,1 / 6$, 1] mon. (c) possible stacking sequence with unit cell (a) and (b). .165

Figure 7.27 Projective views along monoclinic b axis (a) unit cell for perfect stacking with $[001]_{\text {mon }}$ stacking vector. (b) unit cell constructed for revealing another oxygen layer faulting structure with c' stacking vector. (c) possible stacking sequence with unit cell (a) and (b). .166

Figure 7.28 Total 12 calculated X-ray intensity curves with different oxygen layer faulting probabilities (ranging from 0\% 100\%).

Figure 7.29 Total 12 calculated X-ray intensity curves for (a) Oxygen layer fault (b) Metal layer faulting with different stacking probabilities (ranging from 0\% 100\%)....168 Figure 7.30 Total 12 calculated X-ray intensity curves with different "anti-phase" oxygen layer faulting probabilities (ranging from 0\% 100\%). .170

Figure C1.1 Comparison between different residual error forms $R_{w}$ and $R_{p}$ defined in (C1). .203 


\section{Preface}

My PhD work was supervised by Prof. Y.U. Wang in the Department of Materials Science and Engineering, and part of this work has been published in 2 peer reviewed papers and 1 conference proceeding:

Section 3.2 and 3.3 in Chapter 3 are reprinted from the paper [W.F. Rao, K.W. Xiao, T.L. Cheng, J.E. Zhou, Y.U. Wang, Applied Physics Letters, 97 (2010) 162901].

In this paper, W.F. Rao and K.W. Xiao performed most simulations; T.L. Cheng participated in discussion and manuscript revision; I did simulations and participated in data analysis and manuscript revision; Y.U. Wang participated in data interpretation and did critical manuscript revision.

Section 2.1 in Chapter 2, section 3.4 in Chapter 3 and Appendix A are reprinted from the paper [J.E. Zhou, T.L. Cheng, Y.U. Wang, Journal of Applied Physics, 111 (2012) 024105].

In this paper, I performed simulations, participated in theoretical analysis and manuscript drafting; T.L. Cheng contributed to useful discussions and manuscript revision; Y.U. Wang did theoretical analysis and critical manuscript revision.

Section 2.2 and 2.4 in Chapter 2 and part of contents in Chapter 4 are reprinted from the conference proceeding [J.E. Zhou, Y.U. Wang, EPD Congress 2014, John Wiley \& Sons, Inc., 2014, pp. 299-307]. Not included in the proceeding are comparison experiments (SEM and X-ray diffraction) performed by Y. Yan and S. Priya in Virginia Tech., which are summarized in section 4.3 in Chapter 4.

In this proceeding, I performed simulations and participated manuscript drafting; Y.U. Wang participated in data interpolation and critical manuscript revision.

Collections of Chapter 5, 6 and 7 will be submitted for publication in:

J.E. Zhou, Y.U. Wang, "Effects of Crystallographic Texture and Grain Shape Anisotropy on Piezoelectric Properties of Ferroelectric Polycrystalline Ceramics,"

J.E. Zhou, Y.U. Wang, "Phase Field Modeling of Textured Ferroelectric Polycrystals. Part II: Dielectric and Piezoelectric Properties of Template-Matrix Composites,"

J.E. Zhou, Yang Ren, Yongmei M. Jin, Y.U. Wang, "Analysis of $x \mathrm{LiCoO}_{2}-(1-x) \mathrm{Li}_{2} \mathrm{MnO}_{3}$ Lithium Ion Battery Cathode Materials: A Hierarchical Structural Refinement Approach."

In these draft papers, I perform simulations, data analysis and article drafting; Y.U. Wang participated in data analysis and critically revised manuscripts; Dr. Ren and Dr. Jin participated in group discussions, contributed to useful suggestions and manuscript revision.

Jie Zhou

July 2015 


\section{Acknowledgement}

My sincere acknowledgement should first be given to my advisor, Professor Yu U. Wang, who serves not only as supervisor for my $\mathrm{PhD}$ thesis, but also as a mentor for my future career. During the valuable time I spent with Prof. Wang in the past 5 years, he enlightened me to the interesting and mystical world of computational material science, taught me essential knowledge and tools which will also play an important part in my future work, and most importantly, encouraged me for independent and critical thinking. Impacted by his persistence and inspiration, instead of easily giving up, I learnt to insist on finding solutions when I encountered challenging problems. Such a great experience is invaluable for my life. An old Chinese proverb best summarizes the achievement I obtained from my advisor: "Give a man a fish and you feed him for a day. Teach him how to fish and you feed him for a lifetime."

I would like to give my deep gratitude to Prof. Yongmei M. Jin, who spared no pains to assist me with complex code development and selflessly share every single detail of her experience on simulations. Apart from the technique supports and fruitful discussions offered by Prof. Jin, encouragement from her really acted as healing walks through my hard times and made me optimistic when feeling depressed.

I would like to thank Prof. Jaroslaw W. Drelich, Prof. Stephen A. Hackney, Dr. Yang Ren (Argonne National Lab) and Prof. Yongmei M. Jin for serving as my committee members and for helpful suggestions on my research. Especial thanks are given to Dr. Yang Ren for providing the funding to support my battery research and long distance travel for my defense. I really appreciate Prof. Mark R. Plichta, Prof. Peter D. Moran, Prof. Paul G. 
Sanders and Prof. Douglas J. Swenson of their good advice and help for my research progress. I want to thank Prof. Stephen L. Kampe for his kind and impressive help. Thanks to Prof. Jiann-yang Hwang, Dr. Bowen Li and Dr. Zhiwei Peng for the help and useful information on my career. Also, I would like to thank Dr. Edward A Laitila, Owen P Mills, Ruth I. Kramer, Paul Fraley for their experimental supports and thank Beth R Sickler and Margaret P Rothenberger for managing miscellaneous paper works for me.

Happy times with all my labmates and friends in the beautiful town Houghton is always memorable. They are Dr. Tianle Cheng, Dr. Fengde Ma, Liwei Geng, Weilue He, Dr. Jingtuo Zhang, Dr. Hui Wang, Fei Li, Yu Wang and others not fully listed here. Special thanks to my former schoolmate and friend Shanshan Xu now in Stanford University, for our "academia debates" and his timely encouragement which benefit me a lot.

Most importantly, I would like to express my deepest gratitude to my parents Mr. Zhongmin Zhou and Mrs. Yanli Liu for their love, support and understanding. I know they did lots of things for me more than I could imagine, and such strong family bond is my motivation to move on. I hope they will be gratified for the accomplishment of this thesis.

Finally, funding supports are acknowledged: the study of ferroelectric materials was supported by DOE under Award No. DE-FG02-09ER46674; the parallel computing was performed on XSEDE supercomputers under Grant No. TG-DMR100033; the diffraction analysis of lithium ion battery cathode materials was supported by Argonne National Lab under Contract No. 3F-31541 and 4F-30001; and use of Advanced Photon Source, an Office of Science User Facility operated for U.S. DOE Office of Science by Argonne National Lab, was supported by DOE under Contract No. DE-AC02-06CH11357. 


\section{Abstract}

Ferroelectric materials, as a large family exploited for the application of sensors, transducers and random access memories, open up a remarkable ground both for fundamental science and industry. Dielectric and piezoelectric properties are of the most interest in ferroelectric materials, which motivate research to enhance ferroelectric properties based on various application purposes. Among the multitudinous candidates in ferroelectric family, pseudo binary solid solutions with $\mathrm{ABO}_{3}$ lattice structure attract special attention in virtue of their large strain response when applying external loading. Furthermore, existence of morphological phase boundary (MPB) on their phase diagrams shed light on tuning material compositions to improve ferroelectric properties as well. Essentially, polarization domain properties play the key role in ferroelectric property enhancement, which need further investigations.

This thesis primarily focuses on the computational study of microstructure-property relation in representative ferroelectric material: $\mathrm{Pb}\left(\mathrm{Zr}_{1-\mathrm{x}} \mathrm{Ti}_{\mathrm{x}}\right) \mathrm{O}_{3}$ (PZT). For its single crystal, domain engineering and ferroelectric nucleation mechanisms are studied. And for its polycrystal, grain texture development during sintering, grain shape effect, and twophase composites are investigated to exploit their effects on domain evolution and further property enhancement.

Phase field modeling is developed to simulate domain evolution in PZT single crystal and polycrystal. Extensive simulations show that wisely selecting electric field could design domain patterns, while purposely choosing operation temperature and electric field magnitude could realize domain size control. In addition, theoretical study shows that 
ferroelectric nucleation presents a correlated manner which is different from traditional isolated nucleation. For polycrystal studies, the model for templated grain growth (TGG) to generate texture is developed. To validate the model, the simulations are compared to the experiment with good agreement. As accompanied issues, grain shape anisotropy, texture-property relation and second-phase effects are studied with phase field modeling. It is revealed that grain shape plays the minor effect on ferroelectric properties as compared with grain texture, and competition between texture and second-phase results in an optimal volume fraction for template seeds involvement.

Further incorporation of domain microstructures potentially contributes to extraordinary X-ray diffraction peaks, especially when domain sizes shrink to nano-scale. As the candidate for the material who possesses the similar structural feature, layeredstructure lithium ion battery materials present nano-scale structural domain variants with stacking faults. Investigations on the structure of lithium ion battery not only facilitate the understanding of X-ray diffractions related to nano-domain, but also supply the novel methodology to quantitatively study microstructure-property relation. DIFFaX modeling is adopted to reveal its atomic level stacking structure information. And a new hierarchical algorithm is developed to quantitatively obtain the distribution of stacking fault probability which serves as potential evidence to explain that the best performance of battery cathode happens at certain composition. Additionally, instead of focusing on transition layer only, models to deal with possible oxygen faulting and voids are proposed as well to extend the capability of studies for more scientific problems concerning with particular microstructures. 


\section{Chapter 1. Introduction}

\subsection{Ferroelectric Materials}

Ferroelectricity defines the properties that materials have spontaneous electric polarizations, domains and hysteresis loops when applying external electric field [1], which is analogy to its counterpart "ferromagnetism" [2]. Ferroelectric materials will present ferroelectricity when cooling down below Curie temperature. Due to the existence of spontaneous polarization, ferroelectric materials possess dielectric property which describes the polarization response to external applied electric field. Also, due to the effect of electrostriction which couples local polarization with lattice distortion, well aligned polarizations through electric poling present macroscopic strain in ferroelectric materials. Such conversion ability between external electric force and strain response is also called piezoelectricity. There are lots of applications based on dielectric property and piezoelectric property in ferroelectric materials. Based on ferroelectric dielectric property, ferroelectric materials are used as ferroelectric random access memories (FeRAM) []] utilizing the switching ability of polarization states in PZT-based materials, microwave tunable devices [4] and capacitors. Based on the revealed high piezoelectricity, ferroelectric materials are well applied to stabilized piezoelectric resonator [5] , nonresonant transducers used on electrical reproducers and microphones [ $[\underline{6}]$ and the up-todate high technique devices such as microelectromechanical systems (MEMS) [7]], relaxor-

based ultrasound [] $]$, MEMS-tilt sensors [9], actuators [10, 11] and types of motors $[\underline{12}, \underline{13}]$. Motivated by the required performance enhancement and further industrial 
applications, fruitful works were documented to study ferroelectric materials both theoretically and experimentally $[\underline{1}, \underline{14}, \underline{15}]$. Early families of ferroelectric materials present several deficiencies such as chemical instability, structural complexity and very low Curie temperature which prohibits usual applications [16, 17]. Later discovery of ferroelectric materials with perovskite structure (general formula $\mathrm{ABO}_{3}$ ) overcomes these disadvantages with chemical stability, very simple structure and high operating temperature (room temperature). As one of the representatives, $\mathrm{BaTiO}_{3}[\underline{18}]$ is successfully used in the $\mathrm{BaTiO}_{3}$-based piezoelectric transducer working devices functioning with electrical poling. Although $\mathrm{BaTiO}_{3}$ crystals are adopted in many field applications, shortcomings along with depoling and accompanied low coercivity are the facing challenges [19] and virtually limited to only mono-functional usage. Later, the systems of $\mathrm{Pb}\left(\mathrm{Zr}_{1-\mathrm{x}} \mathrm{Ti}_{\mathrm{x}}\right) \mathrm{O}_{3} \quad(\mathrm{PZT}) \quad[\underline{15}], \quad \mathrm{Pb}\left[\left(\mathrm{Zn}_{1 / 3} \mathrm{Nb}_{2 / 3}\right)_{1-\mathrm{x}} \mathrm{Ti}_{\mathrm{x}}\right] \mathrm{O}_{3} \quad(\mathrm{PZN}-\mathrm{PT}) \quad[\underline{20}] \quad$ and $\mathrm{Pb}\left[\left(\mathrm{Mn}_{1 / 3} \mathrm{Nb}_{2 / 3}\right)_{1-\mathrm{x}} \mathrm{Ti}_{\mathrm{x}}\right] \mathrm{O}_{3}(\mathrm{PMN}-\mathrm{PT})$ [21] stand out as the representatives of perovskite type solid solutions. Phase diagrams of such three pseudo-binary systems shown in Fig. 1.1 present the peculiar phase boundary called morphotropic phase boundary (MPB) separating regions of tetragonal phase and rhombohedral phase by a nearly temperature independent vertical line. The accompanied inspiring phenomenon that composition in the vicinity of MPB presents much better piezoelectric properties is discovered [모 $\underline{21}, \underline{22}]$. New monoclinic phase [ $\underline{23}]$, nanodomain [24], and two phase coexistence [25] are the three primary attempts to explain such enhancement around MPB in the purpose to give impetus to better understanding of intrinsic effect in single crystals. Thus, the ability to tailor 
piezoelectric property by tuning composition causes both the industrial interest and motivation to study the underlying mechanisms.

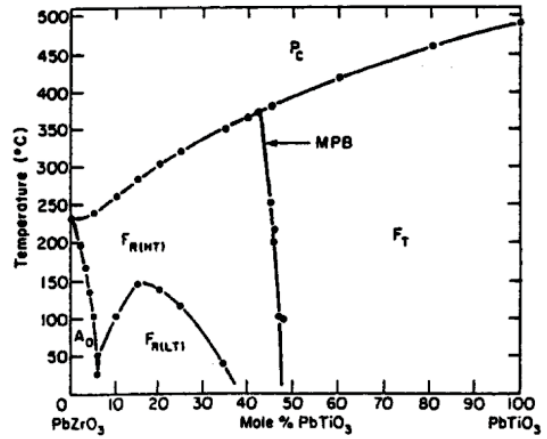

(a) PZT

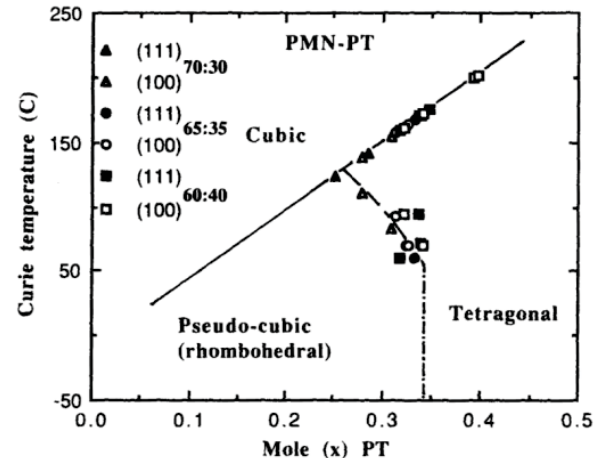

(b) PMN-PT

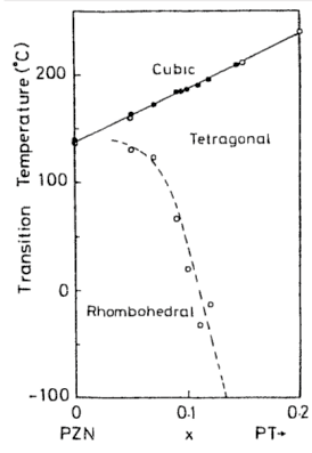

(c) PZN-PT

Figure 1.1 Phase diagram for (a) PZT [14]. (b) PMN-PT [22]. (c) PZN-PT [20]. For copyright permissions, refer to Appendix F.

Although single crystals offer ideal prototypical model to exploit the anisotropy properties of best performance, their practical uses are limited to several difficulties: (i) for some system such as PZT, to obtain single crystal is hard due to incongruent melting point of $\mathrm{ZrO}_{2}$ [14]; (ii) for systems as PMN-PT and PZN-PT, single crystals are available however costly. Recently, instead of exploiting structural homogeneous single crystal, more and more works now focus on materials with heterostructures such as polycrystalline ferroelectrics [26], different phase/composition multilayers [27], multiferroics composites [28, 29], nanoscale physics [30] and PZT thin films [31] on account of developing new novel materials by involving coupling effect among different participant phases. Many established techniques such as tape casting/doctor-blading, sol-gel process [32], ionic dopants [플 pave the way for manufacturing the required multicomponent heterostructures in ferroelectric ceramic science and engineering [34]. No matter what kind of 
heterostructures in materials, ceramic form (polycrystal) is of the most common morphology. Fig. 1.2(a) shows the grain morphology of equiaxed polycrystal obtained from computer simulation for illustration purpose. If grains are with randomly orientated crystallographic axes, materials will exhibit quite poor performances, fortunately however, when crystallites assembly prefer to align along certain direction (uniaxial texture as defined with preferred grain orientations as shown in Figs. 1.2(d-1) to (d-3)), the properties are enhanced compared with the case of randomly oriented grains $[\underline{35}, \underline{36}]$. With the stimulation to generate high textured polycrystal samples, especial those of high piezoelectric coefficients when in single crystal form, fabrication routine named templated grain growth (TGG) [37] is developed to realize such goal and successfully applied to many ferroelectric systems [38] to obtain quite promising characteristics. Fig. 1.2(b) shows templated grain structure during TGG process. In addition, grain shape would be elongated as shown in Fig. 1.2(c) during TGG. Therefore, texture, template seeds and grain shape all play a role in materials property, leading to an even more complex manner. Either the enhancement origin coming from intrinsic properties in single crystal form or additionally from the external coupling issues in polycrystal requires deep investigations. In the following computational studies, PZT is primarily adopted in our model due to its well documented experimental data and representativeness. 


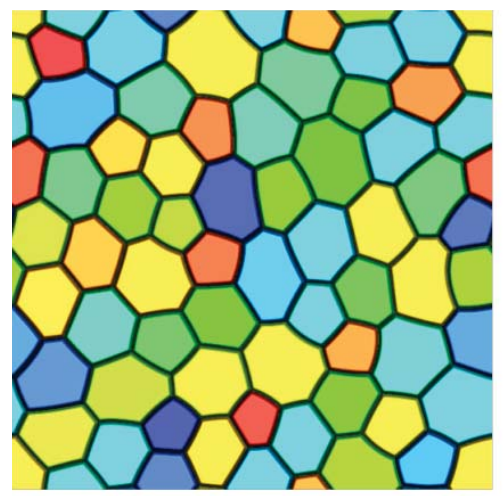

(a)

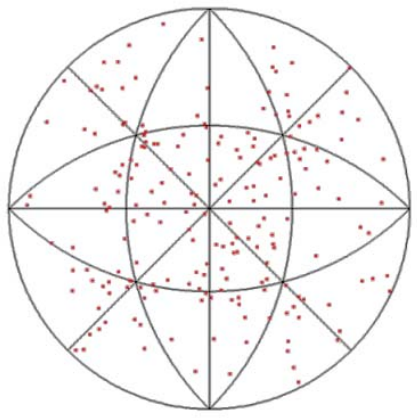

(d-1) Untextured $\left(90^{\circ}\right)$

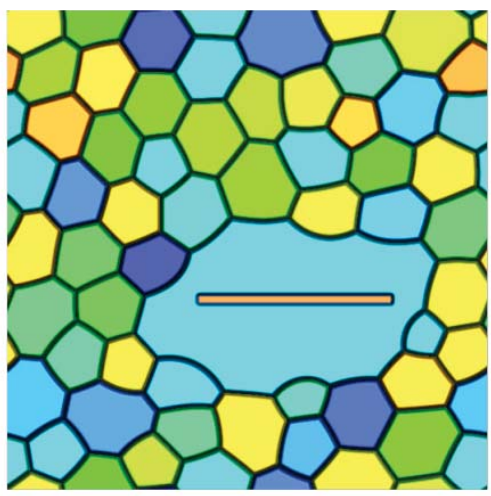

(b)

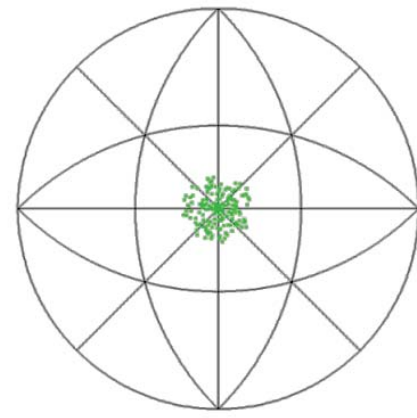

$(d-2)$ Textured $\left(20^{\circ}\right)$

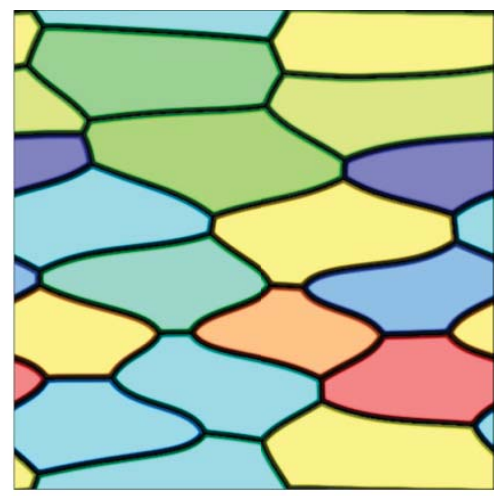

(c)

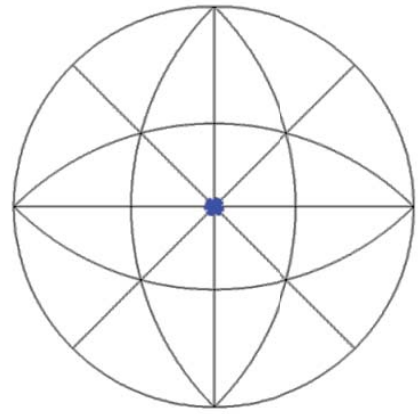

(d-3) Textured $\left(5^{\circ}\right)$

Figure 1.2 Simulated Grain Structures for (a) equiaxed polycrystal (b) polycrystal with template seed (c) polycrystal with elongated grain shape. Stereographic projections of (010) plane grain orientation distributions for $(\mathrm{d}-1)$ untextured $\left(90^{\circ}\right)(\mathrm{d}-2)$ intermediately textured $\left(20^{0}\right)$, and (d-3) highly textured $\left(5^{0}\right)$ grains.

\subsubsection{Domain Mechanisms for Dielectric and Piezoelectric Behaviors in Ferroelectrics}

As mentioned previously, ferroelectricity will present spontaneous polarization when cooling down cross Curie temperature. In the ideal infinite single crystals, spontaneous polarization could be reasonably assumed uniformly aligned. Such antetype model is favorable only for theoretical deduction, however, in real crystals, due to existence of surfaces and defects, spatial heterogeneous distribution of polarization is more realistic instead of uniform polarization assumption, except for the rare application in 
semiconducting ferroelectrics with single domain state [39]. The region within which the polarizations are of the same magnitude and orientation is defined as one single domain. So, multiple-domain coexistence due to polarization heterogeneity in real ferroelectric materials is the common fact that will automatically take complexity into material behaviors related with polarization distributions. Polarization heterogeneity (domain structure) either on surface or in the bulk introduces depolarization field which could prevent the macroscopic net polarization resulting in very poor piezoelectricity [1]. Realizing the importance of domain structure, the opinion of "better to see" leads to many experimental techniques $[\underline{1}, \underline{40}]$ being developed for the visualization of ferroelectric domain structures. For bulk characterization, optical birefringence, second-harmonic generation [41], electron microscopy [녀] etc. are commonly used, in addition, for surface or superficial layers characterization, chemical related methods [43-45], liquid crystal method [46], X-ray diffraction [47], and photoemission are usually adopted.

The origin of ferroelectricity is the spontaneous displacements of B-site atoms and oxygen atoms in the unit cell of perovskite crystals. Fig. 1.3 schematically shows the typical different ferroelectric phases [48] transformed from non-ferroelectric cubic phase with exaggerated accompanied lattice distortions. For each possible phase, there are several crystallographic equivalent polarization directions (e.g. 6 for tetragonal) leading to diverse equilibrium domain patterns. As intrinsic property in ferroelectrics, domain structure is essential characteristic that significantly affects ferroelectric properties such as dielectric constant and piezoelectricity. Such two important properties are highly linked with local polarization distributions through intrinsic relations [49]. 
As stated before, multi-domain structure is commonly formed to minimize the depolarization field, electric and elastic energies due to the existence of surfaces. The boundary to separate different domains apart is called domain wall [드 whose properties are another important issue. With the help of intersection angle between polarizations of two adjacent domains, domain wall type could be defined by such angle, for example, $90^{\circ}$ and $180^{\circ}$ domain walls existing in tetragonal phase. To obtain dielectric property using small signal measurements [51], and piezoelectric property using standard resonance techniques [52], both the techniques require external electric/stress signals which could be a driving force for domain wall motion when coming up with large magnitude of measuring input. As an example in polycrystalline study, the ferroelectric properties obtained from implicit method by statistically averaging of intrinsic single crystal data $[\underline{53}, \underline{54}]$ deviate from explicit measurements because of the ignorance of domain wall motion. As also pointed out in early literatures $[\underline{55}, \underline{56}]$, dielectric, piezoelectric and their aging effects are affected by domain wall motion. So, the extra contribution from non- $180^{\circ}$ domain wall motion (e.g. $90^{\circ}$ walls) needs to be purposely addressed as extrinsic effect [49] in addition to intrinsic effect where no domain wall motion is involved.

Possible domain structures in single crystals are well studied by symmetry changes during phase transformation $[\underline{57}, \underline{58}]$ with the motivation that ferroelectric properties as hysteresis loops, remanence and coercivity are highly depend on domain structure [59-61]. Recent finding on high piezoelectric response along non-polar direction in single crystal with engineered domain near MPB [ㄷ] once again stimulates the investigation on its origin. The tailored microstructure of domains could be obtained from domain engineering 
techniques [63, 64]. According to previously mentioned interpolation by averaging intrinsic single domain properties, piezoelectric anisotropy with other engineered domain structures [65-67] could be partially explained [68-70] by converting material constants tensor to required non-polar directions. However, neither the mismatch enhancement between experiment and calculation model nor the domain size effect $[\underline{69}, \underline{71}]$ could be well explained stimulating other explanation attempts such as domain wall broadening mechanisms [72] based on reversible interphase transformation happened on the specific static domain wall. No matter what the advanced explanations would be, the domain configuration in single crystal is always their standpoint. Domain structure in polycrystalline is quite different from single crystal because each individual grain is imposed with internal stress through contacted boundaries in order to preserve macroscopic shape of grains. Domain structure tries to accommodate itself with a collaborative way by forming electric and elastic twinning to further eliminate free energy. Based on the systematical studies $[\underline{73}, \underline{74]}$, in fine-grained polycrystals [두], simple lamellar domain structure is preferred, while, in coarse-grained systems, a more complex banded twin structure [76] is favored. Both of the two cases impose the influence of domain structure and domain wall motion on dielectric and piezoelectric properties. Investigation [77] on $\mathrm{BaTiO}_{3}$ and PZT systems demonstrates that nearly $60 \% \sim 70 \%$ of piezoelectricity originated from domain wall motion. The $P$-E and $\varepsilon$-E hysteresis loops as the characteristic curves for the ferroelectric performance are the direct reflection of domain wall motion effect for large signal measurements. Electric treatment before use for ferroelectric ceramics is an equivalent way to do domain engineering and could obtain significant enhancement with 
roughly factor of two because numerous non- $180^{\circ}$ domain walls are generate [78, $\underline{79}$ ]. More importantly, the mobility of domain wall in different phases (e.g. tetragonal and rhombohedral) and even its mechanical feature causing materials shape change [으 are diverse so that the poled materials experienced a selection of primary domains to acquire large dielectric and piezoelectric response $[\underline{49}, \underline{81}]$. Additionally, theoretical study [르 $]$ on PZT close to MPB shows that grain size also plays a role for selection of tetragonal and rhombohedral domains. As several more examples from industrial cases, many actuators highly rely on the changes of domain structure with external electric field [49] and multilayer ceramic capacitors (MLCCs) [83] are strongly affected by domain wall motion. Therefore, domain structure and domain wall motion contribute significantly to electromechanical behavior of ferroelectric materials in every aspect, and at the same time, involve in more complexities and nonlinearities to such problem, which actively requires deep studies.
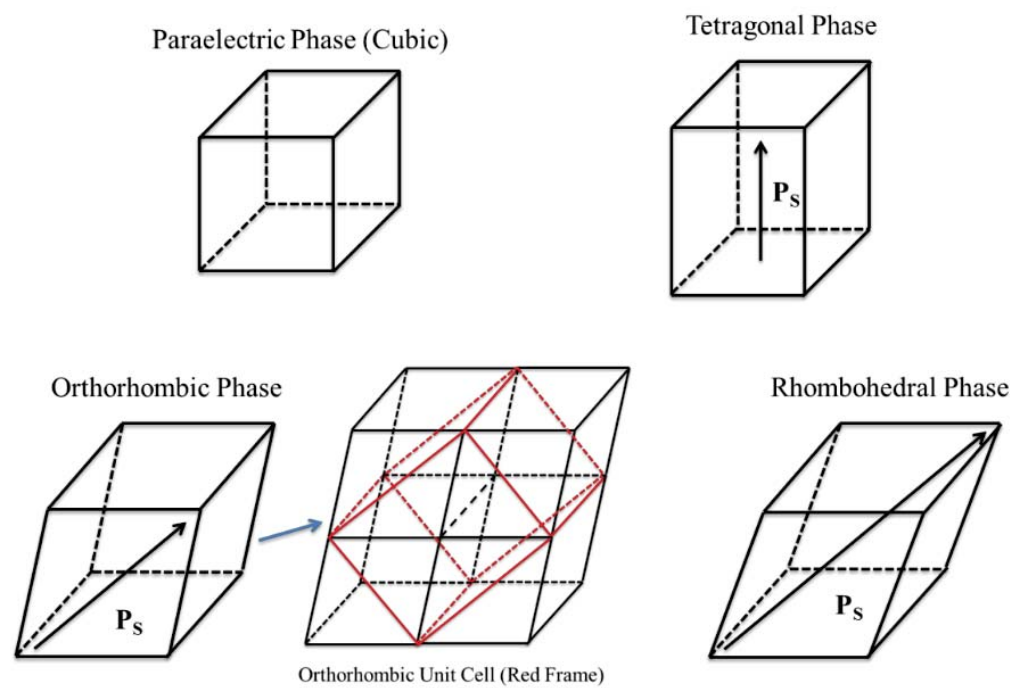

Figure 1.3 Schematic lattice geometries for common ferroelectric phases and spontaneous polarization alignment. 


\subsubsection{Domain-Level Modeling and Simulation Method: Phase Field Model}

Early attempts based on the domain-level modeling used to predict electromechanical properties are on the assumption of fixed domain wall and free of other defects. By simply assuming grain shape to be spherical and domain in the grain to be single, polycrystalline properties are calculated by averaging on these single crystals with additionally boundary condition considerations such as stress between internal grain and surroundings [뇨]. Further improvement with more complex structure in single crystallite (e.g. existence of laminar $90^{\circ}$ and $180^{\circ}$ domain walls) instead of single domain configuration [ $[\underline{85}]$ is implemented. More precise models $[\underline{73}, \underline{74}, \underline{86}, \underline{87}]$ concerning with crystalline orientations, domain size and configuration are developed by equivalently averaging dynamic property (domain wall motion) into single crystallite.

Compared with previous models, the so called micromechanical model based on the ad hoc criteria [88] plays a part on the improvement for more "dynamic" character like polarization switching under external load. Its improvements using Reuss approximation $[\underline{89}, \underline{90]}$ and extension for more complex domain configurations [91] are validated [92] by accurately reproducing hysteresis loops. By adapting effective medium approximation (EMA), a self-consistent treatment $[\underline{91}, \underline{93}]$ is exploited to take account of the coupling among grains with Eshelby inclusion method. Other treatments [뇨, $\underline{66}, \underline{94}, \underline{95}]$ emphasizing on domain compatibility conditions based on continuous assumption across domain wall are alternative ways to predict possible compatible domain structures $[\underline{76}, \underline{96}, \underline{97}]$ thus leading to different dynamic properties. All these models are based on 
constitutive laws with the requirement of premeditate domain structure and evolution, which facilitates the implement in finite element computations [98-100]. Furthermore, there is a typically continuous treatment called sharp-interface model [101] to describe the microstructure and its evolution with interfaces between different phases. Physics laws are equivalently considered assembly to be a driving force to move interface forward with certain rate, thus, it turns out to be a problem of tracking of moving boundaries.

Nevertheless, all these early static or quasi-dynamic models have their own drawbacks as the experiment results are usually significantly underestimated or the realtime dynamic of domain wall motion could not be captured respectively. Before evolved into well-defined domain structure during phase transformations, ferroelectric material quenching from high temperature undergoes correlated nucleation stage $[102, \underline{103}]$ which serves as embryo for further developed configuration of domains. A more "neural" model to integrate dynamics linking nucleation and self-accommodating domain evolution is highly called for. Although sharp-interface scheme could track the interface boundary, domain structure might develop into quite complex structure with topology even changed, resulting in tedious front tracking algorithm. Phase field model emerge as the times require as an efficient approach for evolution of microstructures. The fundamental energy functional is formed based on classic works by predecessors [104-106]. Such model depicts ferroelectric domains by a set of order parameters [107] taking discriminated values in different domain regions. Mechanisms like boundaries evolution are resolved by representing different energy terms with order parameters including bulk energy, domain wall energy, electrostatic energy and elastic energy and minimizing the constituent free 
energy. Instead of sharp-interface description for domain wall, a diffused interface is adopted within the transition region by continuously but rapidly varying order parameters. Unlike treating the different phase separately in the sharp-interface model, diffused phase field model integrates all the phase contributions together in a coherent way leading to elegant formulas which provide us with a more simple description of realistic physics and free interface tracking that reducing the effort of the numerical implementation to much lower extent and extending the studies of microstructures to even more complex ones. Aiming at the ferroelectric materials, primary order parameters are naturally chosen to be spontaneous polarization and suitable formulism is developed accordingly [108]. Difference among phase field models is mainly upon the treatment of energy terms. For example, for the elastic energy term, so called phase field microelasticity (PFM) theory are developed possessing the ability to conveniently treat elastic and structural inhomogeneous systems using Fourier transform $[\underline{109}, \underline{110]}$. Moreover, to simulate domain evolution, time-dependent Ginzburg-Landau (TDGL) equations $[111, \underline{112}]$ are solved using Fourierspectral method [113] with high efficiency. By adding extra Langevin noise terms [114] into TDGL equations could further investigate the thermal fluctuations and then model nucleation phenomenon without any pre-assumptions on the feature configuration of nuclei [115]. Diffused interface phase field models are powerful not only because of supplying us with all the critical requirements for modeling ferroelectric domain evolution, but also applying to many meaningful realistic physical processes as solidification [116], solid state phase transformations [117], grain growth [118], crack propagation and voids $[119, \underline{120}]$, domain evolution in thin films [121], dislocation dynamics [122] and many other 
applications [107]. Also such richly endowed tools pave a way to find new lead-free environmental-friendly materials [123] by the prophecy [124] that new ferroelectric materials will be uncovered by predictive theory and modeling.

\subsubsection{Texture Development and Computational Diffraction}

As stated previously, ferroelectric polycrystals act as the potential substitutes for single crystals which are usually not available due to intrinsic fabrication difficulties. In order to obtain the anisotropy properties for polycrystal materials, grain texture needs to be introduced. Templated grain growth (TGG) supplies such feasible way for grain texture development during sintering. For some ferroelectric materials such as system of PMN-PT matrix with $\mathrm{BaTiO}_{3}$ templates, $\mathrm{X}$-ray diffraction technique is adopted at different sintering stages [125] to gain the grain structural information: texture development. Continuously tracing the sintering process gives better understanding and control on microstructural evolutions. In-situ X-ray diffraction is the most competent technique, for example to study the growth of crystallites [126]. However, such facility requires complex set up and advanced software supports which make it unavailable in common laboratories. Furthermore, the application of experimental XRD has its own limitation such as the mismatch of penetration depth on certain constituent structure [127]. The fact motivates us to perform computational X-ray diffraction based on statistical consideration of assembly of grain orientations. Grain structures simulated by phase field modeling supply the evolution of grain morphology with time during sintering, at the same time, X-ray diffraction calculation is conducted by considering feature orientations for textured grains through diffraction theory for powders. Development of Lotgering factor is calculated with 
time to characterize texture evolution. Thinking of Lotgering factor as the measure of volume fraction of "new textured phase" (new textured grains) with time, similar curve as Johnson-Mehl-Avrami plot is constructed to show Lotgering factor development for sintering process. The bottom-up calculation supplies a feasible way to simulate in-situ Xray diffraction to study texture development, thus, paves the way to investigate materials for which experimental in-situ X-ray facility might not be available.

Further incorporation of structural distortions in addition to grain structures potentially contributes to extraordinary diffraction peaks, especially when domain sizes shrink to nano-scale [128]. The possible peculiar phenomena is usually beyond the scope of X-ray detection resolution, therefore, calculation for adaptive X-ray diffraction phenomenon [24] makes up for such deficiency and increases the simulation accuracy for

novel materials. As the candidate for the material who possesses such adaptive X-ray diffraction phenomenon, layered-structure lithium ion battery materials present nano-scale structural domain variants with stacking faults. Investigations on the structure of lithium ion battery not only facilitate the understanding of X-ray diffractions related to nanodomain, but also supply the novel methodology to quantitatively study microstructureproperty relation. In the following section 1.2 , we will focus on lithium ion battery cathode materials.

\subsection{Lithium Ion Battery Cathode Materials}

Lithium ion battery brings the great impact on our daily life spanning from efficient transportation like hybrid engines [129] to many electronic developments and applications 
[130]. As another functional material with structural heterogeneities, lithium ion battery cathode acting as important constituent part of the battery itself causes enormous interests and innovation for the whole branch of lithium ion battery industry [131] which is accompanied with the improvements on electrolyte and anode materials as well. As shown in Fig. 1.4, the working principle for charge and discharge of lithium ion battery is illustrated. In a simplistic way, such two reverse processes are corresponding to the extraction and insertion of lithium ions respectively from cathode which is typically comprised of oxides of transition metals with variable valence state [132], while for anode, carbon-based compounds are usually adopted. Candidacies such as $\mathrm{LiCoO}_{2}[\underline{133}], \mathrm{LiNiO}_{2}$ [134], $\mathrm{LiMn}_{2} \mathrm{O}_{4}\left[\underline{135}\right.$ ] vanadium oxide $\mathrm{LiV}_{3} \mathrm{O}_{8}$ [136] and phosphates $\mathrm{LiMPO}_{4}$ [137] with various substitution element $\mathrm{M}$ (Fe [138], Mn [139], Co [140]) are the most common materials used as cathode. Charging voltage and capacity are the two main benchmarks to judge the battery performance. In order to either balance such two factors or unilaterally enhance one, additional transition metals are inserted into materials resulting in changing of lithium composition, for example, $\mathrm{Li}\left(\mathrm{Ni}_{1 / 3} \mathrm{Mn}_{1 / 3} \mathrm{Co}_{1 / 3}\right) \mathrm{O}_{2}[\underline{141}], \mathrm{Li}\left(\mathrm{Mn}_{1.5} \mathrm{Ni}_{0.5}\right) \mathrm{O}_{4}$ [142] and $\mathrm{Li}(\mathrm{Mn}, \mathrm{Fe}, \mathrm{Co}) \mathrm{PO}_{4}[\underline{143}]$. Further developed applications with doping [131, 144] are the alternative ways to tailor the compositions to obtain improved performances. As stated previously, lithium ions are extracted from cathode during charging, which could lead to lattice parameter change [145] accompanied with generation of internal stress and cracks [146]. Moreover, performance of battery highly depends on the interface properties between electrode and electrolyte, thus, to promote intercalation on the interface [147] and to fabricate the stabilized layered structure by involving second phase to form 
"composites" help to improve capacity retention. Typical composites are obtained through combining $\mathrm{LiMO}_{2}$ cathode materials with monoclinic $\mathrm{Li}_{2} \mathrm{MnO}_{3}(C 2 / \mathrm{m})[\underline{148}, \underline{149}]$ or with spinel structured $\mathrm{LiMn}_{2} \mathrm{O}_{4}[\underline{150}, \underline{151]}$ as the stabilized geometry. To improve the performance of lithium ion battery, factors as in plane ordering of transition metals, intercalation during cycling and stability are all falling into one junction: microstructures of cathode materials which require further investigations.

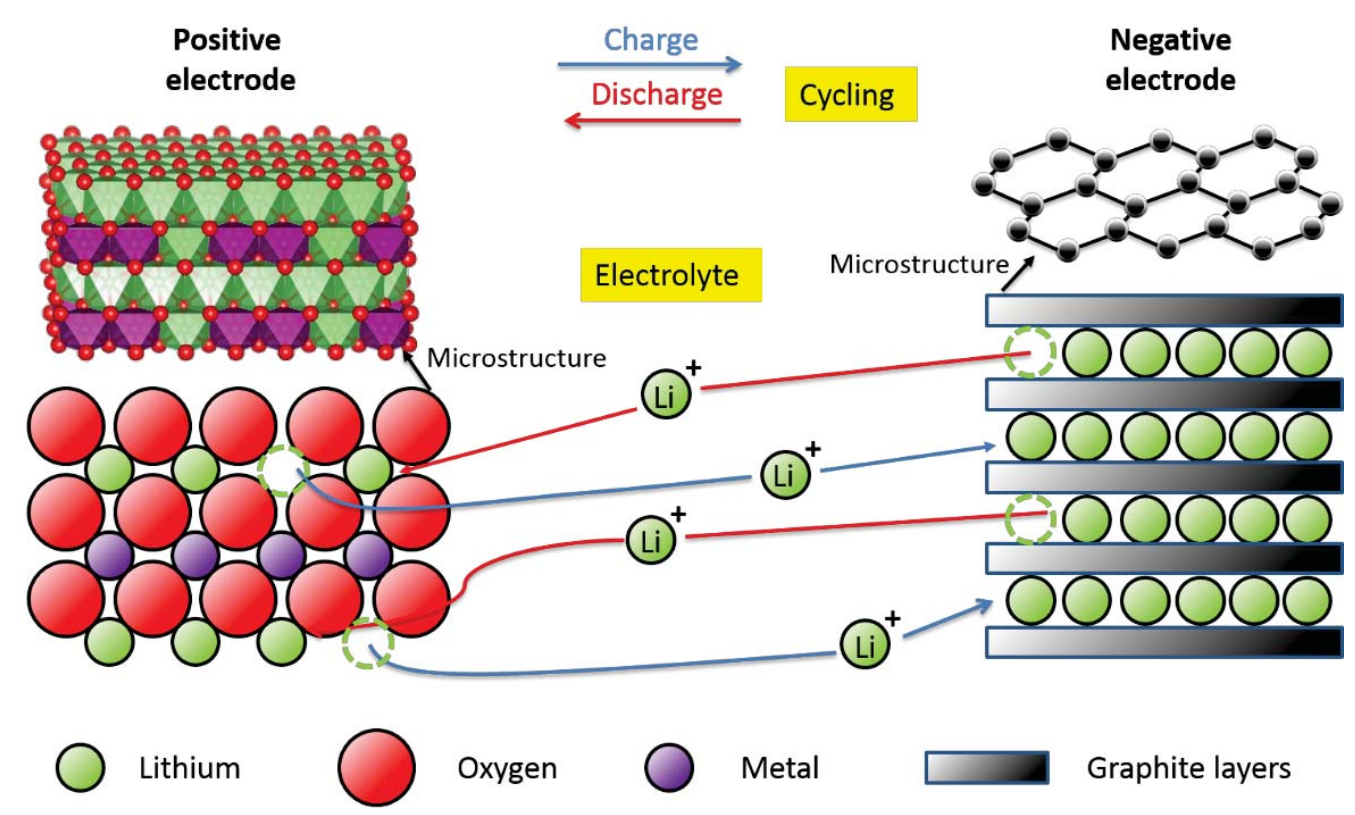

Figure 1.4 Adapted from ref. [152] to demonstrate the working principle in lithium ion battery.

\subsubsection{Capacity Fade Phenomenon of the Cathode Materials}

Capacity fade refers to the capacity attenuation during battery cycling, usually accompanied with increasing impedance as well. Commercial lithium ion batteries suffer the fade up to $40 \%$ during several hundreds of cycling [153]. Due to attendant side reactions, the cause of fade attributes to many mechanisms such as electrode mass 
unbalancing [154], overcharge states for electrodes with deposition and decomposition processes $[154,155]$, electrolyte decomposition reactions on lithium metal surfaces [156], film formation at interface [157], electrode (positive) dissolution [158]. What is more, during insertion/distraction of lithium, lattice expansion or phase change (e.g. structural degradation in spinel structure causing the fade problem [159]) on electrodes could happen. In particular, overdischarged state on positive electrode turns on Jahn-Teller distortion effect [160] which induces large cell expansion to prevent intergrowth structure in single crystal from maintaining stable. The relevant phenomenon also happens on carbon electrodes during cycling causing interlayer spacing change along $\mathrm{c}$ axis [161] and stacking structure change [162]. Many early mathematical models developed for lithium-ion batteries aim at better understanding of their behaviors, however, capacity fade processes are absent in these incomplete descriptions stimulating more sophisticate modellings [163]. Involving capacity fade mechanisms into investigation is the key point to improve our understanding on cycling of lithium battery, thus, to better understand capacity fade phenomenon, microstructures of cathode materials deserves well studies. Based on such motivation, electron diffraction [164], high resolution synchrotron X-ray diffraction combined with high resolution S/TEM facilities [ $\underline{165}]$, neutron diffraction [166] are widely adopted to exploit the underlying structures of lithium battery materials. Compared with intercalation compound $\mathrm{LiCoO}_{2}$, lithium manganese oxides as good candidates are cheaper and safer which trigger the potential replacement for $\mathrm{LiCoO}_{2}$. In such manganese oxides compounds, the oxidation of manganese plays the key role in extraction of lithium process in order to keep the charge neutrality in cathode. As a contrast to the traditional idea that 
Mn could not be oxidized beyond +4 valence state [167], new finding reported further oxidation of $\mathrm{Mn}^{4+}$ in $\mathrm{Li}_{2} \mathrm{MnO}_{3}$ system [168] which enhances electrode potentials in real battery application and also supplies the good prototype for clarifying fundamental argument that it is rather due to Mn's redox process than simply to availability of $\mathrm{Li}^{+}$ions that performance of battery cycling is affected [169]. Although as a common problem, $\mathrm{Li}_{2} \mathrm{MnO}_{3}$ itself has relatively poor cycleability [170], novel intergrowth of $\mathrm{Li}_{2} \mathrm{MnO}_{3}$ and $\mathrm{LiMO}_{2}\left(\mathrm{M}\right.$ represents transition metals) better improves the performance with $\mathrm{Li}_{2} \mathrm{MnO}_{3}$ acting as the role of stabilizing framework of electrode structure during cycling [148]. Structural information on $\mathrm{Li}_{2} \mathrm{MnO}_{3}$ and its intergrowth composites is worth of deeper studies based on well-documented scientific works.

\subsubsection{Nanoscale Structural Defects: Stacking Fault and Ordering}

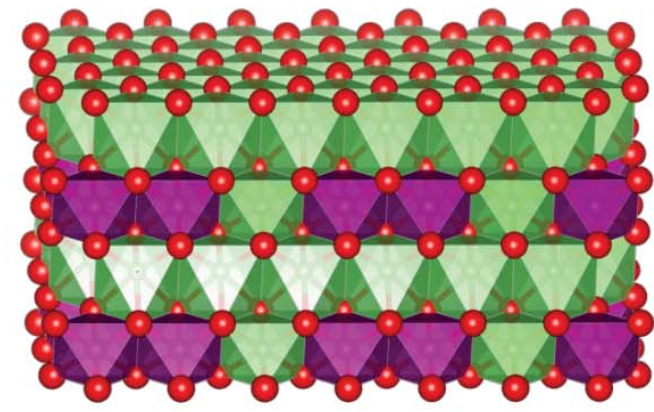

(a)

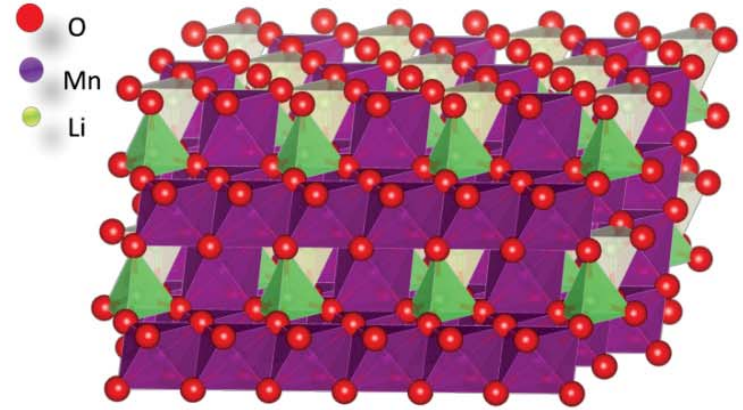

(b)

Figure 1.5 (a) Visualized $\mathrm{Li}_{2} \mathrm{MnO}_{3}$ layered structure. (b) Visualized $\mathrm{LiMn}_{2} \mathrm{O}_{4}$ spinel layered structure.

Fig. 1.5 shows the typical layered structures for monoclinic $\mathrm{Li}_{2} \mathrm{MnO}_{3}$ with space group $C 2 / \mathrm{m}$ and spinel $\mathrm{LiMn}_{2} \mathrm{O}_{4}$ with space group $\mathrm{Fd} 3 \mathrm{~m}$, both of which are already well established by many works [171-174]. O layers are piled up with the framework of FCC 
close packing ( $\mathrm{ABC}$ sequence), and $\mathrm{Mn}$ occupies octahedral sites designated with purple polygons in Fig. 1.5, while designated with green polygons, Li occupies octahedral sites in $\mathrm{Li}_{2} \mathrm{MnO}_{3}$ and tetrahedral sites in $\mathrm{LiMn}_{2} \mathrm{O}_{4}$ depending on the excess of composition $\mathrm{Li}$. As classical thermodynamics shows us that certain involvement of disorder is expected to obtain equilibrium, the existence of imperfect stacking faults similar to point defects and dislocations is not surprising in 2-D planar structures as in layered structures. Some battery materials have larger amount of stacking faults generated with random or non-random way [175] especially in layered $\mathrm{Li}_{2} \mathrm{MnO}_{3}$ system. Fig. 1.6 illustrates the possible stacking faults in layered $\mathrm{Li}_{2} \mathrm{MnO}_{3}$. It is worth to note that the feasible stacking vectors satisfy the requirement of not changing $\mathrm{O}$ framework resulting in the alternative shift of Mn layers by different stacking vectors (all the possible stacking vectors are labeled in Fig. 1.6). Known as Warren fall [176], "Saw-tooth" like X-ray diffraction peaks related to faulting effect are investigated with variant amount of stacking faults [177] resulting in different peak shape features in certain $2 \theta$ range. As stated before, novel intergrowth of $x \mathrm{Li}_{2} \mathrm{MnO}_{3} \cdot(1-x) \mathrm{LiMO}_{2}$ materials are fabricated to improve battery performance later with an unexpected finding that certain composition $\boldsymbol{x}$ results in the best electrochemical properties [178]. By involving more composition of $\mathrm{Li}_{2} \mathrm{MnO}_{3}$, on the one hand, it helps to improve the layered feature to prohibit phase change of components during cycling, on the other hand, capacity loss is increasing [179]. So, composition of $\mathrm{Li}_{2} \mathrm{MnO}_{3}$ is critical for battery performance. Stacking fault is highly related with composition in the way that tailoring $x$ might affect the stacking fault distribution; therefore, information of stacking fault should be purposely addressed. 
The first successfully synthesized two phase layered $\mathrm{Li}_{2} \mathrm{MnO}_{3}-\mathrm{LiCoO}_{2}$ battery materials in 1997 are proposed as solid solution [180, 181], including later fabrication of $\mathrm{Li}_{2} \mathrm{MnO}_{3}-\mathrm{LiFeO}_{2}$ system [182]. Instead of solid solution notation, a new opinion related with the transition metal layer ordering is proposed [148]. As an example, novel complex compound $x \mathrm{Li}_{2} \mathrm{MnO}_{3} \cdot(1-x) \mathrm{LiMn}_{1 / 3} \mathrm{Ni}_{1 / 3} \mathrm{Co}_{1 / 3} \mathrm{O}_{2}$ presents transition metal layer flowerlike ordering with discernable differences on prescribed X-ray diffraction peak shape. To these two point of views, solid solution notation focuses on the mechanism of exchanging elements belonging to different layers, leading to the treatment of assigning occupancy probability, while, in-plane ordering notation bases on the nanoscale intergrowth between the two constituent phases with exact element occupancy. Actually, stacking fault without changing $\mathrm{O}$ framework as discussed before could be seen as an alternative way of ordering because stacking fault vectors only vary the relative positions of transition metal layer to other layers. It is also worth to mention that as traditional viewpoint, total disordered structure should demonstrate chemical inertness instead of showing unexpected well electrochemical property [179]. Ordering feature seems more intrinsic when solid solution notation faces more and more challenges among the debate between the two opinions. To clarify which notation is right, characterization information such as X-ray diffraction and transition-metal-sensitive neutron scattering technique is required in order to capture the evidence of the actual structure. It deserves further studies on such structural variants in the purpose to better understand battery materials behavior. 


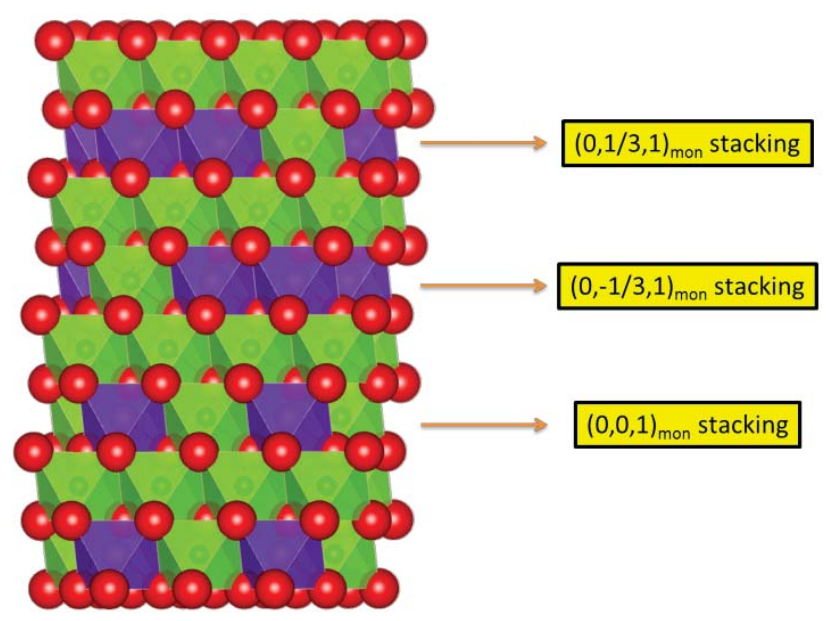

Figure 1.6 Stacking faults for layered $\mathrm{Li}_{2} \mathrm{MnO}_{3}$ structure with translation of stacking vectors.

\subsubsection{Nanoscale Computational Diffraction Method: DIFFaX}

To quantitatively obtain the structural information related with stacking fault or ordering, several computational methods are exploited with their own advantages. Firstprinciples calculations focus on the dynamic structural changes during cycling reaction by comparing energy differences between hypothetical states $[\underline{165}, \underline{183}, \underline{184]}$. It is powerful in the feasible chemical reaction, defect dynamics and optimization of atomic occupancy in the unit cell. However, first-principles are bottom level algorithms which have both computational limitations on the faulting structure and physical complexity with the treatment of atomic pseudopotentials. The requirement for up level treatment to link X-ray diffraction with structural information such as faulting and ordering stimulates the generation of the theoretical formulations [185-187]. To facilitate the calculation, software is developed based on theoretical deductions. As one of the software candidates, although traditional rietveld refinements could refine the structure based on the prescribed 
hypothetical unit cell model, the lack/inefficient way of considering stacking fault leads to the relatively poor fitting. Based on the involvement of transition probabilities among different layers, both DIFFaX [188] and FAULTS [189] well estimate the percentage of faulting information as quantitative method. Additionally, FAULTS could refine the parameters encountered in rietveld refinements and interlayer transition probabilities as well. To some extent, FAULTS shows more quantitative feature, however, the main drawback for FAULTS is much more computational resources and time consuming than other two. Furthermore, in spite of that DIFFaX could only do the good job in qualitative way, we develop a new strategy based on DIFFaX (presented in Chapter 7) to quantitatively study the stacking fault distribution, supplying a potentially more efficient algorithm. So, $\mathrm{DIFFaX}$ is adopted to investigate stacking fault problems in our objective systems.

\subsection{Objectives and Thesis Arrangements}

The objectives of this thesis aim at developing phase field models applied to ferroelectric materials of single crystal, polycrystal, and two phase composites forms. Microstructural features as domain evolution, grain texture development, grain shape anisotropy and two phase composite arrangement are systematically investigated in order to exploit microstructure-property relations to obtain enhanced ferroelectric properties. As another notable material with astonishing microstructures such as stacking fault and ordering, lithium ion battery cathode also presents microstructure-performance relations during cycling. It is worth to mention that frontier research unfolders an innovative idea that nanotwin supperlattice structure exists in ferroelectric materials near MPB and such 
nanoscale domains cause adaptive X-ray diffraction phenomenon [24]. Both MPB-based ferroelectric materials and layered lithium ion battery materials possess nanoscale domain level microstructure, therefore, an intrinsic analogy between them imperatively enlighten us to focus on structural change and domain evolutions, naturally leading to the common characterization method: high resolution X-ray diffraction. In spite of different methods to do investigation on such two quite different systems, the intrinsic similarity and common objective to engineer domain structure adequately prompt us to investigate them in this thesis.

The details of phase field models, relative computational X-ray diffraction theory and recursive algorithm for stacking fault are presented in Chapter 2. Easy directions of polarization for different phases are of significance to investigate the topology of domain patterns. With the help of free energy landscapes belonging to different phases in single crystal, domain mechanisms are simulated and more importantly, as precursor for domain evolution, correlated nucleation in prototypical ferroelectric single crystal is theoretically studied and simulated as well in Chapter 3. Motivated by meaningfully involving textured polycrystals, templated grain growth process and related grain texture development are simulated in Chapter 4. Inevitably, templated grain growth might induce shape anisotropy and different extent of textures. Thus, ferroelectric properties against grain texture and shape are studied in Chapter 5. Usually, to obtain highly textured polycrystals through templated grain growth, secondary inferior phases are chosen as template texture controllers, which at the same time produce composite effect. The domain mechanisms to uncover the competition between texture and inferior volume fraction are discussed in 
Chapter 6 aiming at optimization of controlling volume fraction of secondary phases. In Chapter 7, new strategy based on DIFFaX is developed and applied to $x \mathrm{Li}_{2} \mathrm{MnO}_{3} \cdot(1-$ $\boldsymbol{x}) \mathrm{LiCoO}_{2}$ systems to quantitatively obtain the distribution information of stacking faults. With the same computational model, microstructural characterization related with in-plane order/disorder in lithium ion battery cathode is investigated as well. All these structural studies on battery cathode are summarized. Finally, Chapter 8 presents the summary for the thesis and my further research. 


\section{Chapter 2. Computational Methodology*}

In this chapter, phase field model for single-phase ferroelectric materials will be first presented for the study of domain evolution in ferroelectric single- and poly-crystals. Phase field models for templated grain growth and two-phase ferroelectric composites are then developed to investigate the microstructure-property relations. Finally, X-ray diffraction calculation models corresponding to different length scales, namely, coarse grains and atomic layer intergrowth, are introduced to study the texture development in polycrystals during templated grain growth (TGG) process and stacking faults phenomenon in layered structures.

\subsection{Phase Field Model of Ferroelectric Materials}

Phase field model for ferroelectric materials provides a tool to investigate domain evolution in such systems and response to external field (i.e. electric field and stress).

Homogeneous ferroelectric system is well described by using Landau-GinzburgDevonshire theory $[\underline{105}, \underline{190}]$. Function of polynomial form $f(\mathbf{P})$ characterizes the nonequilibrium local bulk free energy density of a stress-free ferroelectric state in terms of position-independent polarization vector $\mathbf{P}$ as $[\underline{105}, \underline{190]}$ :

\footnotetext{
* Part of texts in section 2.1 is from J. Appl. Phys., 111, 024105-1-13, 2012. Refer Appendix D for granted permission to be republished. Part of texts in sections 2.2 and 2.4 is from EPD Congress 2014, John Wiley \& Sons, Inc., pages 299-307. Refer Appendix E for granted permission to be republished.
} 


$$
\begin{aligned}
f(\mathbf{P}) & =\alpha_{1}\left(P_{1}^{2}+P_{2}^{2}+P_{3}^{2}\right)+\alpha_{11}\left(P_{1}^{4}+P_{2}^{4}+P_{3}^{4}\right)+\alpha_{12}\left(P_{1}^{2} P_{2}^{2}+P_{2}^{2} P_{3}^{2}+P_{3}^{2} P_{1}^{2}\right) \\
& +\alpha_{111}\left(P_{1}^{6}+P_{2}^{6}+P_{3}^{6}\right)+\alpha_{112}\left[P_{1}^{4}\left(P_{2}^{2}+P_{3}^{2}\right)+P_{2}^{4}\left(P_{3}^{2}+P_{1}^{2}\right)+P_{3}^{4}\left(P_{1}^{2}+P_{2}^{2}\right)\right], \\
& +\alpha_{123} P_{1}^{2} P_{2}^{2} P_{3}^{2}
\end{aligned}
$$

where the expansion coefficients $\alpha_{i}, \alpha_{i j}$, and $\alpha_{i j k}$ are determined by fitting theory to experimental data. In order to study ferroelectric phase transformation which involves inhomogeneous states, position-dependent polarization vector field $\mathbf{P}(\mathbf{r})$ needs to be used. Except for bulk free energy, to describe the behavior of inhomogeneous systems, other energy density terms, such as electrostatic energy, elastostatic energy and polarization gradient energy should be taken into considerations. By summing up all the energy contributions, the total system free energy of an inhomogeneous system can be written as a functional of the field variable $\mathbf{P}(\mathbf{r})[\underline{104}, \underline{108}, \underline{191-193]}$ :

$$
F=\int d^{3} r\left[f\left(R_{i j} P_{j}\right)+\frac{1}{2} \beta_{i j k l} \frac{\partial P_{i}}{\partial r_{j}} \frac{\partial P_{k}}{\partial r_{l}}-P_{m} E_{m}^{e x}\right]+\frac{1}{2} \int \frac{d^{3} k}{(2 \pi)^{3}}\left[\frac{n_{i} n_{j}}{\varepsilon_{0}} \tilde{P}_{i} \tilde{P}_{j}^{*}+K_{i j k l} \tilde{\varepsilon}_{i j} \tilde{\varepsilon}_{k l}^{*}\right]
$$

where summation convention over repeated indices is implied. Texture effect attributed to each grain is considered through grain rotation matrix $R_{i j}$ which describes the geometry (size, shape, location) and crystallographic orientation of individual grains in the polycrystal. The gradient term in Eq. (2.2) represents the polarization change across domain walls, where $\beta_{i j k l}$ is the gradient coefficient tensor. $\mathbf{E}^{\mathbf{e x}}$ is the external applied electric field variable. The last two k-space integrals give the electrostatic energy of polarization distribution $\mathbf{P}(\mathbf{r})$ and the elastostatic energy of electrostrictive stain distribution $\boldsymbol{\varepsilon}(\mathbf{r})$ under homogeneous elastic modulus assumption [109], where $\mathbf{n}=\mathbf{k} / k$ is a unit vector in k-space, $K_{i j k l}=C_{i j k l}-n_{p} C_{i j p q} \Omega_{q r} C_{k l r s} n_{s}, C_{i j k l}$ is the elastic modulus tensor, and 
$\Omega_{i k}=\left(C_{i j k l} n_{j} n_{l}\right)^{-1}$ is the Green function tensor of elasticity. The superscript asterisk * indicates complex conjugate. The spontaneous stain $\varepsilon$ in ferroelectrics acts as secondary order parameter which is obtained by electrostriction coefficient tensor $Q_{i j k l}$ :

$\varepsilon_{i j}=Q_{i j k l} P_{k} P_{l}$,

Time-dependent Ginzburg-Landau equation [104] in Eq. (2.4) is employed to describe polarization evolution and domain microstructure formation

$\frac{\partial P_{i}(\mathbf{r}, t)}{\partial t}=-L_{i j} \frac{\delta F}{\delta P_{j}(\mathbf{r}, t)}+\xi_{i}(\mathbf{r}, t)$,

where $L_{i j}$ is kinetic coefficient tensor, and $\xi_{i}(\mathbf{r}, t)$ is Gaussian-distributed Langevin noise term to account for the effect of thermal fluctuation. Fast Fourier transform algorithm is used to solve time-dependent Ginzburg-Landau equation.

\subsection{Phase Field Model of Templated Grain Growth}

To simulate TGG process (including grain growth process) in the later chapter, we employ the phase field model of grain growth [118] by reformulating a new Landau-type polynomial potential function and taking into account the special features of TGG as compared with conventional grain growth. In the phase field model, the grain structure in a polycrystal is described by a set of long-range order parameter field variables, $\left\{\eta_{\alpha}(\mathbf{r})\right\}$, whose total number is equal to the number of grains in the polycrystal before grain coarsening. The total system free energy is a functional of $\left\{\eta_{\alpha}\right\}[118]$ : 
$F=\int d^{3} r\left[f\left(\left\{\eta_{\alpha}\right\}\right)+\sum_{\alpha} \frac{1}{2} \beta\left|\nabla \eta_{\alpha}\right|^{2}\right]$,

where $f\left(\left\{\eta_{\alpha}\right\}\right)$ is a Landau-type nonequilibrium local bulk free energy density function that describes the thermodynamic state of multiple grains in a polycrystal. In this work, we use the following multi-well polynomial energy function:

$f\left(\left\{\eta_{\alpha}\right\}\right)=A\left[\sum_{\alpha}\left(3 \eta_{\alpha}^{4}-4 \eta_{\alpha}^{3}\right)+\sum_{\alpha \neq \alpha^{\prime}} 6 \eta_{\alpha}^{2} \eta_{\alpha^{\prime}}^{2}\right]$

whose minima are degenerated at $\left\{\eta_{\alpha}\right\}=\{1,0, \ldots, 0\}$ corresponding to the energetically equivalent orientations of individual grains, where $A$ is an energy scaling parameter. The gradient terms in Eq. (2.5) characterize the energy contributions from grain boundaries, where $\beta$ is gradient coefficient. The grain growth process is described by the spatialtemporal evolution of $\left\{\eta_{\alpha}(\mathbf{r}, t)\right\}$, which is governed by the time-dependent GinzburgLandau equation [118]:

$\frac{\partial \eta_{\alpha}(\mathbf{r}, t)}{\partial t}=-L \frac{\delta F}{\delta \eta_{\alpha}(\mathbf{r}, t)}$

where $L$ is kinetic coefficient. To simulate TGG process by using above-formulated phase field model of grain growth, we consider some special features of TGG associated with the templates, as compared with conventional grain growth. Templates are well-oriented single-crystalline seeds purposely introduced to reorient other matrix grains thus induce texture development through grain growth. To facilitate the control of template seed orientations, the templates should meet certain shape and size requirements. In particular, to achieve [001] texture, plate-shaped templates of large aspect ratio are used, whose plate 
surfaces are parallel to (001) crystallographic plane with lateral dimensions much greater than the plate thickness and particle sizes of the matrix materials; such platelet templates are readily oriented during doctor blade tape casting process, with their [001] axes well aligned along the tape normal direction. In order to reorient the neighboring matrix grains, the crystal lattices of the templates and matrix phase must allow them to epitaxially grow onto each other to achieve common orientation. Since the lateral sizes of plate-like templates are much greater than the more equiaxed matrix grain sizes, individual templates are surrounded by multiple matrix grains, and it is the neighboring matrix grains that nucleate and epitaxially grow onto the templates, while the templates maintain their original orientations. As a result of such TGG process, matrix grains are reoriented to achieve the templates' well-controlled orientation, leading to texture development. While templates of the same phase as the matrix grains are an ideal choice, single-crystalline templates of required shapes and sizes are not always available or cannot be economically fabricated, thus templates of different materials are usually used, as long as heteroepitaxial growth takes place between the templates and matrix grains, and interdiffusion between template and matrix phases does not occur or is negligible during sintering in order to maintain the matrix grains' composition and properties. For example, barium titanate $\mathrm{BaTiO}_{3}$ (BT) can serve as templates to synthesize textured PMN-PT by TGG. Therefore, the isolated "inert" templates do not take active part in grain growth process; instead, they induce heterogeneous nucleation and heteroepitaxial growth of neighboring matrix grains onto them that rapidly merge into each other to form big grains of the same orientation as the templates. Such big grains will subsequently grow at the expense of other smaller 
matrix grains via coarsening process, which converts more and more matrix volume into the well-controlled template orientation, increasing the degree of texture in the polycrystals. Taking into account the above discussed TGG features in the phase field model of grain growth, Eq. (2.7) is solved only for matrix grains to describe their growth and coarsening processes, while the long-range order parameter fields for template seeds are not evolved, which characterize the "inert" template grains during TGG. With this minor modification, the above formulated phase field model of grain growth is employed to simulate the grain structure evolutions in polycrystals during TGG process.

\subsection{Phase Field Model of Two-Phase Composites}

Since two-phase composites form via TGG, phase field model has been developed for ferroelectric composite materials to account for such multi-phase coexistence. In this thesis, we develop a phase field model of polycrystalline ferroelectric. Consisting of matrix grains and template seeds, the state of a ferroelectric polycrystal is described by a polarization field $\mathbf{P}(\mathbf{r})$, whose total system free energy under externally applied electric field $\mathbf{E}^{\text {ex }}$ is already defined by Eq. (2.2). A phase field variable $\eta(\mathbf{r})$ is introduced to describe the phase distribution, which is equal to 1 in the matrix phase and 0 in the template phase. The LGD coefficients are conveniently expressed as $\alpha(\mathbf{r})=\eta(\mathbf{r}) \alpha_{\mathrm{M}}+[1-\eta(\mathbf{r})] \alpha_{\mathrm{T}}$, where $\alpha_{\mathrm{M}}$ and $\alpha_{\mathrm{T}}$ are the coefficients for the matrix and template phases, respectively. Since the elastostatic energy is evaluated by using Khachaturyan's microelasticity theory that assumes homogeneous elastic modulus, the same isotropic elastic constants are used for both matrix and template phases, which makes 
the polycrystalline composites elastically homogeneous (such an assumption is reasonable for PZT matrix and BT template). The spontaneous lattice misfit strain is coupled to

polarization through electrostriction coefficient tensor $Q_{i j k l}, \varepsilon_{i j}^{0}=Q_{i j k l} P_{k} P_{l}$. As the LGD coefficients $\alpha$, the electrostriction coefficient tensor is also r-dependent, $\mathbf{Q}(\mathbf{r})=\eta(\mathbf{r}) \mathbf{Q}_{\mathrm{M}}+[1-\eta(\mathbf{r})] \mathbf{Q}_{\mathrm{T}}$, where $\mathbf{Q}_{\mathrm{M}}$ and $\mathbf{Q}_{\mathrm{T}}$ are the coefficients for the matrix and template phases, respectively. The evolution of polarization and domain microstructure is also characterized by the time-dependent Ginzburg-Landau equation (2.4). We will consider PZT polycrystalline ceramics (composition $x=0.6$ at room temperature) textured by $\mathrm{BT}$ templates, whose material parameters have been experimentally determined $[194,195]$.

\subsection{Computational Diffraction of Textured Polycrystals}

To characterize the grain texture development in polycrystals during the simulated TGG process, the X-ray diffraction peak intensities are computed for the evolving grain structures as simulated by above described phase field modeling, based on which Lotgering factor [196] is further calculated as a quantitative measurement of uniaxial texture development. Since the grain sizes are larger than the coherence length of probing X-ray radiation, coherent scattering and interference effects do not play a role which are significant for nanodomains [24], thus the X-ray diffraction theory for coarse-grained crystalline materials is employed. The relative integrated intensities of X-ray diffraction peaks from untextured (randomly oriented) grains of a polycrystal is [197]: 
$I_{h k l} \propto\left(1+\cos ^{2} 2 \theta_{\mathrm{B}}\right)\left|F_{h k l}\right|^{2} P\left(\cos \theta_{\mathrm{B}} \sin ^{-2} 2 \theta_{\mathrm{B}}\right)$,

where $\theta_{\mathrm{B}}$ is the Bragg angle of the $(h k l)$ peak, $F_{h k l}$ is structure factor, and $P$ is multiplicity factor for powder diffraction. The term in the first parenthesis in Eq. (2.8) is the polarization factor associated with the unpolarized incident X-ray beam, and the term in the second parenthesis is the Lorentz factor. It is worth noting that Eq. (2.8) cannot be directly applied to calculate the peak intensities of the textured grain structures. In order to compute the diffraction peak intensities of the simulated grain structures as obtained by phase field modeling, the factors in Eq. (2.8) are rearranged into two groups:

$I_{h k l} \propto\left(\frac{1+\cos ^{2} 2 \theta_{\mathrm{B}}}{\sin 2 \theta_{\mathrm{B}}}\left|F_{h k l}\right|^{2}\right)\left(P \frac{\cos \theta_{\mathrm{B}}}{\sin 2 \theta_{\mathrm{B}}}\right)$,

where the term in the first parenthesis is treated analytically as it is (including a trigonometric factor $\sin ^{-1} 2 \theta_{\mathrm{B}}$ out of the Lorentz factor, intrinsically associated with the finite peak maximum and breadth), while the term in the second parenthesis is treated separately through a numerical procedure, which explicitly considers the orientation distributions of individual grains and their volume fractions (in the case of randomly oriented grains this effect is reduced to a geometric factor $P \cos \theta_{\mathrm{B}}$ ) as well as the measurement of diffracted beam intensities by a detector with a finite slit opening (for randomly oriented grains this effect is reduced to another geometric factor $\sin ^{-1} 2 \theta_{\mathrm{B}}$ ), as in a real diffractometer setup. In particular, at the Bragg angle $\theta_{\mathrm{B}}$ corresponding to the $(h k l)$ planes, the $\alpha$ th grain is examined to see if it diffracts X-ray beam into the detector; if it does, set $n_{\alpha}=1$, otherwise $n_{\alpha}=0$; and the contribution from the $\alpha$ th grain to the integrated 
peak intensity $I_{h k l}$ is proportional to $n_{\alpha} V_{\alpha}$, where $V_{\alpha}$ is the volume of the $\alpha$ th grain. Upon examining all grains, the relative integrated peak intensity $I_{h k l}$ is evaluated as:

$$
I_{h k l} \propto\left(\frac{1+\cos ^{2} 2 \theta_{\mathrm{B}}}{\sin 2 \theta_{\mathrm{B}}}\left|F_{h k l}\right|^{2}\right) N_{h k l},
$$

where $N_{h k l}=\sum_{\alpha} n_{\alpha} V_{\alpha}$, which is proportional to the total volume of the grains whose $\{h k l\}$ planes are oriented to diffract X-ray beam into the detector at the Bragg angle $\theta_{\mathrm{B}}$. By repeating this numerical procedure for other Bragg angles, the relative intensities of other peaks are computed.

Based on the computed relative integrated peak intensities $I_{h k l}$, the degree of [001] texture can be quantitatively measured by using the Lotgering factor defined as [196]:

$$
f_{00 l}=\frac{P_{00 l}-P_{0}}{1-P_{0}}
$$

where $P_{00 l}=\sum_{l} I_{00 l} / \sum_{h k l} I_{h k l}, P_{0}=\sum_{l} I_{00 l}^{0} / \sum_{h k l} I_{h k l}^{0}, I_{h k l}$ and $I_{h k l}^{0}$ are the $(h k l)$ peak intensities for the textured and untextured polycrystals, respectively. As seen from its definition, the Lotgering factor measures the volume fraction of the polycrystal grains whose $\{001\}$ planes are aligned in a common orientation (in TGG, $\{001\}$ planes are parallel to the tape casting plane) and is detected by X-ray diffractometer as $(00 l)$ peaks. 


\subsection{Computational Diffraction of Nanoscale Intergrowth of Layered Structures with Stacking Faults}

$\mathrm{DIFFaX}[\underline{198]}$ is an open software based on the recursion algorithm [188] to solve powder diffraction of layered structure with stacking faults. Diffraction patterns are calculated by coherently summing up the interference wavefunctions scattered from planar layers. Fig. 2.1(a) shows the perfect stacking structure layer by layer. For each layer, atomic types, positions and occupations need to be defined in DIFFaX. Stacking vector between two adjacent layers is also required to describe their translation relation. Perfect stacking means that the stacking vectors for all the layers are the same, leading to a perfect crystal structure. While as shown in Fig. 2.1(b), different stacking vectors are introduced into layered structure, resulting in "arrangement disorder" called stacking faults. In most of cases, stacking faults present some statistically features, in other words, stacking faults distributions. In order to capture such information, transition probabilities between two adjacent layers are quantitatively defined. Based on specific consideration, transition probability might vary between different types of layers depending on detailed structural features. So, assigning different sets of transition probabilities to layers is the good way to quantitatively study the stacking faults distributions. Computational algorithm used in DIFFaX treats transition probabilities among layers and scattered wavefunctions with uniform matrix representation, facilitating the efficient implementation. Optimized input architecture in DIFFaX not only well organizes different parameters such as lattice parameters, peak shape functions and other required values, but also supplies a clean way to represent multi-layer transition without tedious duplication. Furthermore, DIFFaX is 
designated to efficiently solve the problems related with 2-D layers (a-b plane) having stacking faults along the third direction (c axis) and supplies a potential way for further consideration of voids. Thus, DIFFaX is very suitable tool to study battery cathode materials which have all the structural concerns mentioned above.

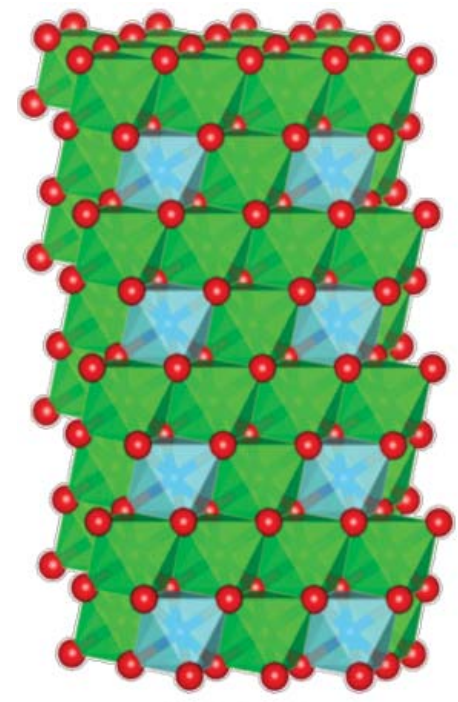

(a) Perfect Stacking

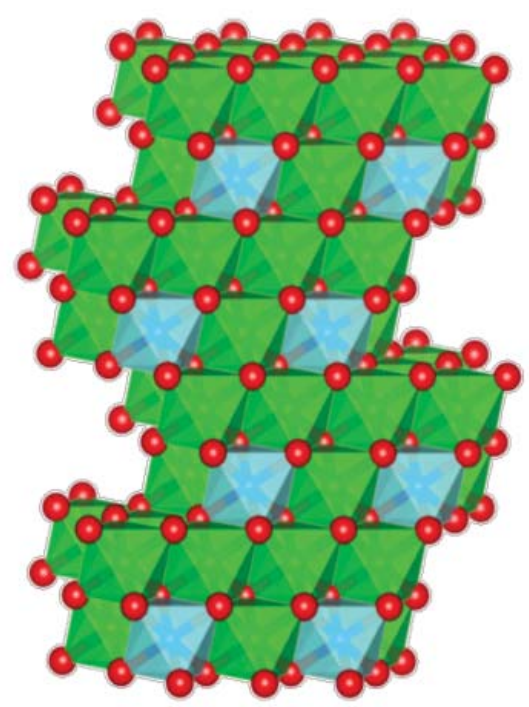

(b) Stacking Faults

Figure 2.1 Layered structures (a) perfect stacking (b) stacking faults visualized by VESTA [199]. 


\section{Chapter 3. Domain Engineering for Ferroelectric Single Crystal ${ }^{\dagger}$}

Piezoelectric response is a material property relating induced internal polarization (or strain) to applied external stress (or electric field) [200]. Such functionalities find wide technological applications as sensors, actuators and transducers [201]. In order to enhance piezoelectric response, single-crystalline materials are often desired [62] because of the piezoelectric anisotropy, where the best property can be obtained along specific crystalline axis. Actually, it is domain structure and domain wall motion in single crystal that significantly affect the properties along specific direction. By wise control of external conditions as called domain engineering, optimized domain configurations could be obtained. Recent experiments show that ferroelectric single crystals with engineered domain configurations exhibit drastically enhanced piezoelectric responses along nonpolar axes $[62, \underline{68]}$. Subsequent experiments further show a domain size effect in the crystallographically engineered ferroelectric crystals [71] where the piezoelectric properties are found to significantly increase with decreasing domain sizes. Our recent computer modeling and simulation reveals a domain wall broadening mechanism [72] that explains the domain size effect in domain engineered ferroelectrics: under electric field applied along non-polar axis of single crystal, domain walls broaden rather than move and serve as embryos of field-induced new ferroelectric phase, producing large, reversible and

\footnotetext{
${ }^{\dagger}$ The contents in this chapter were previously published in Appl. Phys. Lett., 97, 1629011-3, 2010 and J. Appl. Phys., 111, 024105-1-13, 2012. Refer Appendix D for granted permission to be republished.
} 
anhysteretic strain by exploiting the advantageous field-induced inter-ferroelectric phase transformation [108]. The crystallographic domain engineering technique and domain size effect promise to further improve the electromechanical properties by engineering submicron and nanometer-sized domains and develop high-performance, environmentfriendly, lead-free piezoelectrics for sensor, actuator and transducer applications. In order to fully exploit this technique, it is important to understand the mechanisms that control the domain configurations and sizes and find optimal processing conditions for domain engineering. The domain sizes in crystallographically engineered ferroelectric crystals are very sensitive to the experimental conditions during sample treatment. Sophisticated combination of thermal and electrical processing with precise temperature and voltage control has been employed in experiments to engineer the domain configurations and sizes [71], which, however, is still an empirical technique with the underlying mechanisms yet to be fully understood. We employ computer modeling and simulation to investigate the mechanisms for controlling domain configurations and sizes, with a focus on the effects of external electric field applied along the non-polar axis of single crystals during cooling across Curie temperature. The simulations reveal that minimal domain sizes and highest domain wall densities are obtained with intermediate electric field strength, and temperature also plays an important role in domain size control. Investigation on engineered domain points us a way to further enhance material properties.

Domain engineering is usually accompanied with quenching which helps the system for nucleation, then, the whole system experiences phase transformation. So, nucleation stage has its own importance because nucleation could affect the kinetics to 
final domain patterns which requires careful investigations. As one typical solid-state phase transformation, ferroelectric phase transformation exhibits some distinct features from other ferroic phase transformations, such as ferroelastic and ferromagnetic transformations. Unlike ferroelastic transformation that involves only spontaneous strain and elastostatic interaction, ferroelectric transformation involves both spontaneous polarization and spontaneous strain due to electrostriction. Electrostatic interaction plays a dominant role in ferroelectric phase transformation. Such a strong electrostatic interaction imparts some interesting behaviors to ferroelectric phase transformation. As will be shown later, nucleation of ferroelectric phase in the context of classical nucleation theory is impossible, because electrostatic interaction generates an insurmountable energy barrier to isolated nucleus; in order to circumvent such an energy barrier, nucleation exhibits strong spatial correlation and self-organization behaviors from the very beginning, and ferroelectric phase transformation proceeds via spatial and temporal evolution of self-accommodating domains that provide a low-energy kinetic pathway throughout the entire phase transformation process. Such study will give us a better understanding for special nucleation mechanisms in ferroelectric materials. Based on our study on the polarization behaviors of nucleation stage for ferroelectric single crystal, the designed final domain structure, such as stripe domain structure, could be realized by purposely evolving particular external loading environment. 


\subsection{Landau-Type Bulk Free Energy Landscape}

Bulk free energy of ferroelectric materials could be expressed as Landau polynomials as shown in equation (2.1). The landscapes of bulk free energy at different temperatures determine the orientation and magnitude of equilibrium polarizations. Fig. 3.1 shows iso-surfaces of bulk free energy for different phases of PZT. Local regions with stable and metastable energies are designated with yellow labels. At high temperature above Curie point, materials present paraelectric phase with minimum energy located at $\overrightarrow{\boldsymbol{P}}=0$ showing nearly isotropic iso-surface of free energy as shown in Fig. 3.1(a). In paraelectric phase, ferroelectric materials do not present spontaneous polarizations only with randomly distributed small polarizations due to thermal fluctuation without breaking the cubic symmetry. Based on the phase diagram of PZT shown in Fig. 1.1(a), both temperature and composition together determine the equilibrium phase. Fig. 3.1(b) and (c) show the energy iso-surfaces for tetragonal phase and rhombohedral phase respectively by choosing proper combination of temperature and composition. Stable polarizations along the normal of $\{100\}$ family planes and $\{111\}$ family planes obsess the degenerated minimum energies corresponding to tetragonal phase and rhombohedral phase respectively. In the Landau-Ginzburg-Devonshire framework [190], features of different phases could be characterized by experimentally determined temperature dependent coefficient $\alpha_{1}=\alpha_{0}\left(T-T_{0}\right)$ and other coefficients as listed in Table 3.1, which at the same time, also supplies parameters for our later simulations. Topology of bulk free energy is critical for phase identification, domain wall orientation properties and domain structures. 
To intuitively obtain the knowledge of bulk free energy landscape at the first place is very important for us to better understand the kinetic mechanisms during domain evolution.

Table 3.1 Landau coefficients calculated from [195] at room temperature $25^{0} \mathrm{C}$ for PZT. $\mathrm{T}$ and $\mathrm{R}$ represent tetragonal phase and rhombohedral phase respectively.

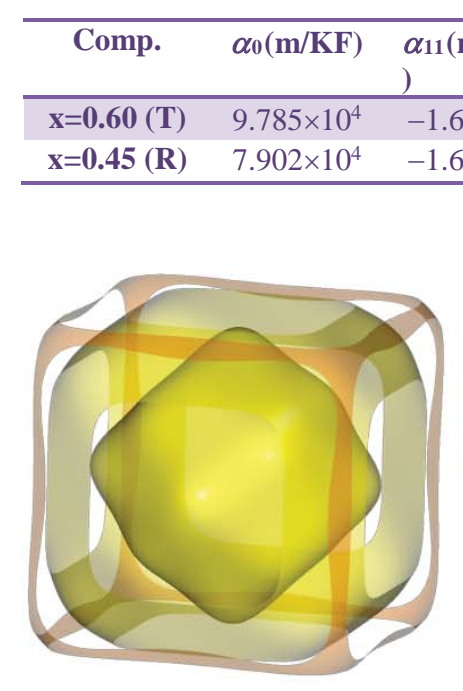

(a)

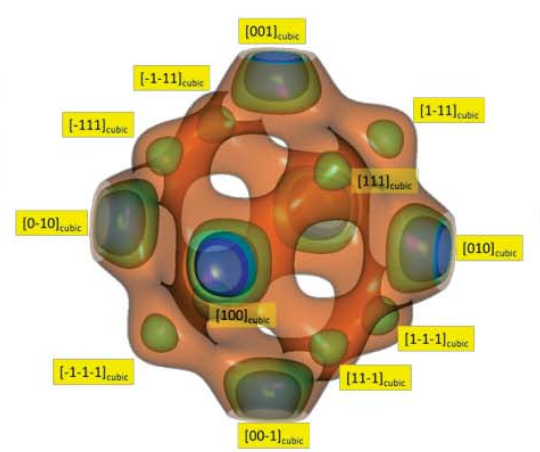

(b)

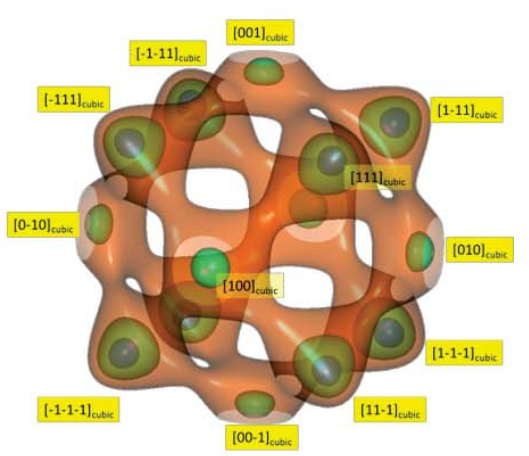

(c)

Figure 3.1 Topologies of bulk free energy iso-surface for different phases in PZT. (a) Cubic phase. (b) Tetragonal phase. (c) Rhombohedral phase. Yellow labels designate different orientations.

\subsection{Phase Field Simulation of Electrical Field Induced Domain Structure Design}

To simulate objective problem, the multi-domain state of a ferroelectric single crystal is described by polarization field $\mathbf{P}(\mathbf{r})$, whose total system free energy under external electric field $\mathbf{E}^{\text {ex }}$ is described by equation (2.2). The experimentally determined Landau-Ginzburg-Devonshire coefficients for $\mathrm{Pb}\left(\mathrm{Zr}_{1-x} \mathrm{Ti}_{x}\right) \mathrm{O}_{3}$ of tetragonal and rhombohedral ferroelectric phase compositions are adopted from Table 3.1. 
The computer simulation shows that application of electric field along non-polar axes is an effective way to control the domain evolution and twin microstructure formation in ferroelectric single crystals, as shown in Fig. 3.2. We consider ferroelectric phase transition (i.e., cooling across Curie point to low temperature) in the presence of external electric field. Fig. 3.2(a) shows the simulated domain formation process in single crystal of tetragonal ferroelectric phase $(<100\rangle$ as polar axes) under electric field applied along [111] direction, which is a non-polar axis of tetragonal crystal. It is found that (110) twins composed of [100] and [010] polar domains (equally favored by [111] electric field) of stable tetragonal ferroelectric phase are formed as the fully-developed engineered domain configuration, which minimizes local bulk free energy through stable tetragonal phase (green), eliminates charge accumulation through head-to-tail polarization patterns, and self-accommodates electrostrictive strain through structural twins. Nevertheless, coexistence of metastable rhombohedral domains (red, blue) are observed along the kinetic pathway of domain evolution, especially at early stage and diminishing with time, as shown in the sequence of Fig. 3.2(a), which is caused by incomplete domain self-accommodation at early stage of domain formation. As shown in previous study [202], phase coexistence through bridging domain mechanism helps reduce the total system free energy of such complex domain microstructures, and provides low-energy kinetic pathways to final engineered domain configurations with complete self-accommodation. Similar domain processes are observed in single crystals of rhombohedral ferroelectric phase $(<111>$ as polar axes) under electric field applied respectively along [100] and [110] directions, which are non-polar axes of rhombohedral crystal. As shown in Fig. 3.2(b,c), (100) twins 
composed of $[1 \overline{1} 1]$ and $[11 \overline{1}]$ polar domains (equally favored by [100] electric field) and (110) twins composed of $[111]$ and $[11 \overline{1}]$ polar domains (equally favored by [110] electric field) of stable rhombohedral ferroelectric phase are respectively formed as the fullydeveloped engineered domain configurations, as also evolved along phase-coexisting lowenergy kinetic pathways. It is worth noting that periodic boundary conditions (both mechanical and electrical) have been used in the simulations, which represent the situations in non-clamped bulk single crystals with surface electrodes to eliminate macroscopic depolarization field. In such cases, it is the kinetics rather than thermodynamics that mainly controls the domain formation and evolution (since global energy minimum would be reached only at a single-domain state). This situation is different from domain pattern formation in thin films that are mechanically clamped by substrates and/or with macroscopic depolarization field, where energy minimization plays a most significant role. It is also noteworthy that the metastable engineered domain configurations need to be stabilized for practical applications. Recent simulations show that short-range ordering of charged point defects developed during ferroelectric aging provides a promising technique for domain stabilization [203].

It is worth noting that above engineered domain microstructures, namely, (100) and (110) rhombohedral twins and (110) tetragonal twins, exhibit average symmetries of monoclinic A, B and C types, respectively [24, 204-206]. With nanoscale domain sizes, these nanodomain microstructures would appear as monoclinic phases in diffraction due to coherent scattering and interference effects $[\underline{24}, \underline{205}, \underline{206]}$. 


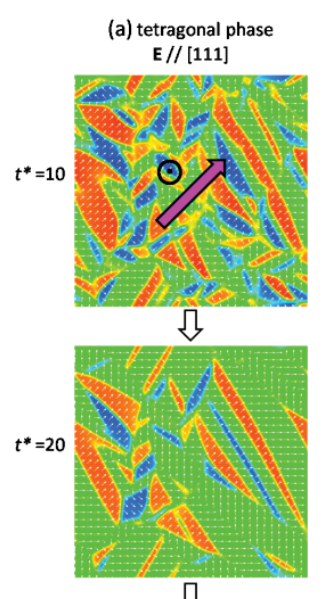

ת
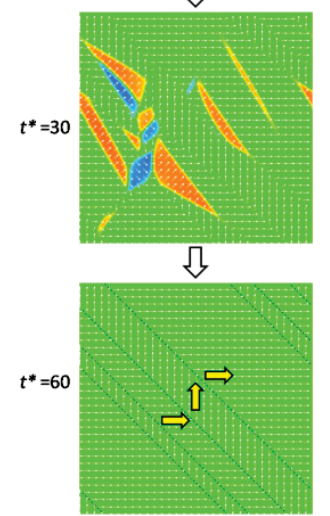

(110) tetragonal twins

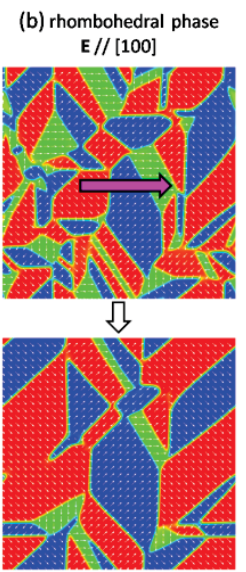

$\sqrt{3}$

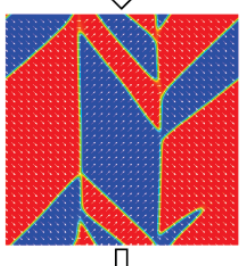

ת

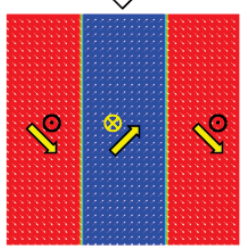

(100) rhombohedral twins

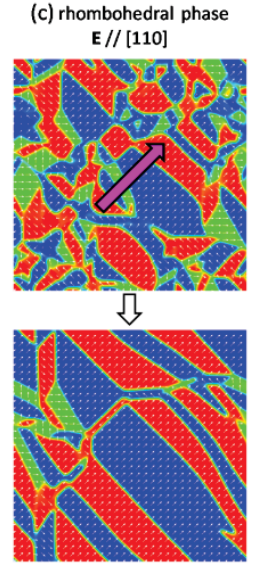

\

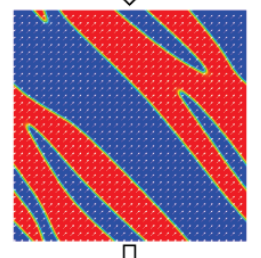

ᄂ

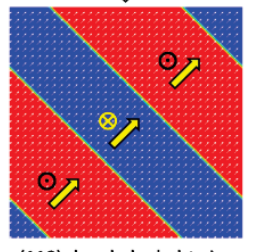

(110) rhombohedral twins

Figure 3.2 Computer simulation of domain evolution and twin microstructure formation in single crystals under external electric field (with field directions highlighted in top row) applied along non-polar axes during ferroelectric phase transition. (a) Tetragonal ferroelectric phase under electric field applied along non-polar [111] direction. (b,c) Rhombohedral ferroelectric phase under electric field applied along non-polar [100] and [110] directions, respectively. Bottom row shows fully-developed engineered domain configurations consisting of structural twins (twin boundaries in tetragonal phase are highlighted by dashed black lines for clarity) with head-to-tail polarization patterns (with polarization directions highlighted in individual domains). 

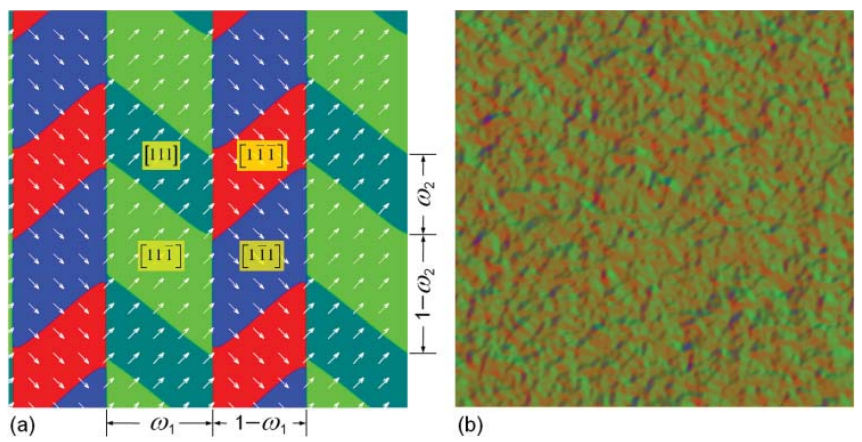

Figure 3.3 (a) Simulated herringbone microstructure under [100] electric field. (b) Simulation of correlated ferroelectric nucleation. Note that polarization length in (b) is less than $0.1 \%$ of equilibrium value in (a).

It is also noteworthy that for rhombohedral crystal under [100] field, herringbone domain microstructures composed of four polar variants $[111],[1 \overline{1} 1],[11 \overline{1}],[1 \overline{1} \overline{1}]$ (equally favored by [100] electric field) are often formed, as shown in Fig. 3.3(a), whose average symmetry is triclinic in general, i.e., average polarization $\langle\mathbf{P}\rangle=P_{R} / \sqrt{3}\left(1,2 \omega_{1}-1,1-2 \omega_{1}-2 \omega_{2}+4 \omega_{1} \omega_{2}\right)$, where $\omega_{1}$ and $\omega_{2}$ are volume fractions of polar domain variants. Diffraction analysis of nanoscale herringbone domains is underway. As a complementary simulation, actually, if applying [100] electric field for rhombohedral phase, there are still several possible final domain configurations. Different resultant cases with size 256 by 256 grids are summarized in Fig. 3.4. All the possible final domain structures come from different energy favorable arrangement of domain variants by [100] electric field. 
electric field along [100] direction with rhombohedral phase
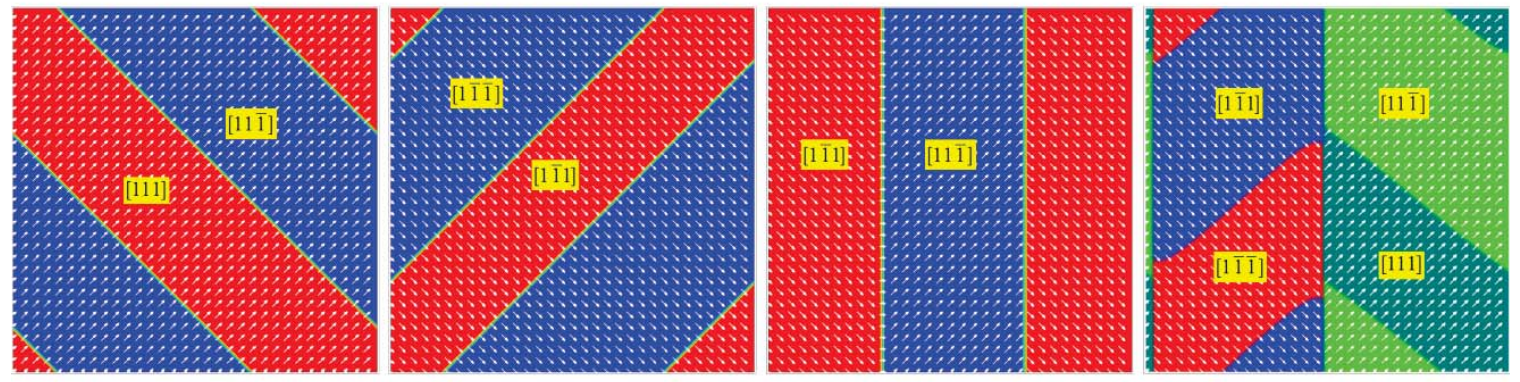

Figure 3.4 Supplementary possible final domain structures when applied with [100] electric field in rhombohedral phase. White arrows represent in plane polarization vector. RGB colors are adopted to show different domains.

\subsection{Phase Field Simulation of Domain Size Control}

The computer simulation further shows a systematic dependence of the domain wall density (or average domain size) in the engineered domain configurations on the magnitude of electric field applied along non-polar axes of ferroelectric single crystals, as shown in Fig. 3.5. We simulate the formation processes of twin-related lamellar domains of stable tetragonal ferroelectric phase under [111] electric field, as in the case of Fig. 3.2(a). The single crystal is quenched across Curie temperature down to $25^{\circ} \mathrm{C}$ in [111] electric field of different magnitudes. For each field magnitude, 15 simulation runs are performed, each with statistically independent thermal noises during domain nucleation. The domain wall densities obtained from the simulations are plotted in Fig. 3.5, with average values highlighted by red filled symbols and solid line. It is found that minimal domain size and highest domain wall density are obtained at intermediate electric field magnitude, while lower and higher fields produce coarser domains thus lower domain wall densities. The simulation shows that selection of polar domain variants by external electric field during 
nucleation stage of ferroelectric phase transition significantly affects the subsequent domain growth and evolution kinetics, thus controlling the formation and sizes of lamellar domains. It is worth noting that the strong electrostatic interaction involved in ferroelectric system generates an insurmountable energy barrier to isolated nuclei, thus nucleation of ferroelectric phase in the context of classical nucleation theory is impossible. In particular, electrostatic energy of a spherical nucleus effectively modifies the quadratic term coefficient of Landau-Ginzburg-Devonshire theory, i.e., $\alpha_{1}^{\prime}=\alpha_{1}+\left(6 \varepsilon_{0}\right)^{-1}$, where $\left(6 \varepsilon_{0}\right)^{-1} \sim 10^{10} \mathrm{~m} / \mathrm{F}$ is orders of magnitude greater than $\left|\alpha_{1}\right|$, prohibiting independent nucleation at any temperature. To circumvent such an energy barrier, ferroelectric nucleation exhibits strong spatial correlation and self-organization behaviors from the very beginning, as shown in Fig. 3.3(b), and ferroelectric phase transformation proceeds via spatial and temporal evolution of self-accommodating domains that provide a low-energy kinetic pathway throughout the entire phase transformation process, as shown in Fig. 3.2. Due to the smallness of $\mathbf{P}$ during correlated nucleation, as shown in Fig. 3.3(b), the free energy difference between the nuclei of stable tetragonal and metastable rhombohedral phases are small; moreover, a small $\mathbf{E}^{\mathrm{ex}}$ does not significantly affect the nucleation because the effect of external field on polar nuclei is proportional to $\mathbf{P} \cdot \mathbf{E}^{\mathrm{ex}}$. Thus, six polar variants of $\langle 100\rangle$ tetragonal domains and eight polar variants of $<111\rangle$ rhombohedral domains nucleate with roughly equal probabilities. As a result, under low electric field, for a given set of lamellar domains, say, composed of [100] and [010] domains forming (110) twins shown in Fig. 3.2(a), the number of [100] and [010] nuclei together is approximately N/7 ( $N$ is the total number of nuclei). To form a (110) domain wall, a pair of [100] and [010] 
nuclei grow into neighboring domains as driven by [111] electric field that equally favors [100] and [010] polar domains, while other nuclei between them are consumed during the domain development process because of either unfavored polar direction or phase metastability $\left(\mathbf{E}^{\mathrm{ex}}\right.$ plays more significant role in domain evolution process due to large $P$ in well-developed domains). Thus, the number of (110) domain walls is estimated to be N/14. With increasing $\mathbf{E}^{\text {ex }}$, the probabilities of different polar nuclei are no longer equal, and more [100] and [010] variants are nucleated, leading to formation of a higher density of (110) domain walls. However, with further increased $\mathbf{E}^{\mathrm{ex}}$, more nuclei of [111] rhombohedral variant are selected, which tend to percolate and form coarse metastable [111] rhombohedral domains that are eventually consumed by stable [100] and [010] tetragonal domains, resulting in coarse (110) twins and low (110) domain wall density. It is the competition between nucleation of [100], [010] and [111] variants under [111] electric field that determines the average domain size and (110) domain wall density, thus maximal domain wall density is obtained at intermediate electric field magnitude, as shown in Fig. 3.5. Such engineered domain configurations with controlled domain sizes provide optimal initial domain microstructures for field-induced inter-ferroelectric phase transformations to exploit desired piezoelectric behaviors.

The computer simulation also shows that temperature is another important factor in domain size control, as shown by blue symbols and line in Fig. 3.5. The crystal is quenched to $250^{\circ} \mathrm{C}$, and the same dependence of domain wall density on field magnitude is observed, except for higher maximal domain wall density at lower electric field strength as compared with the case of $25^{\circ} \mathrm{C}$. 


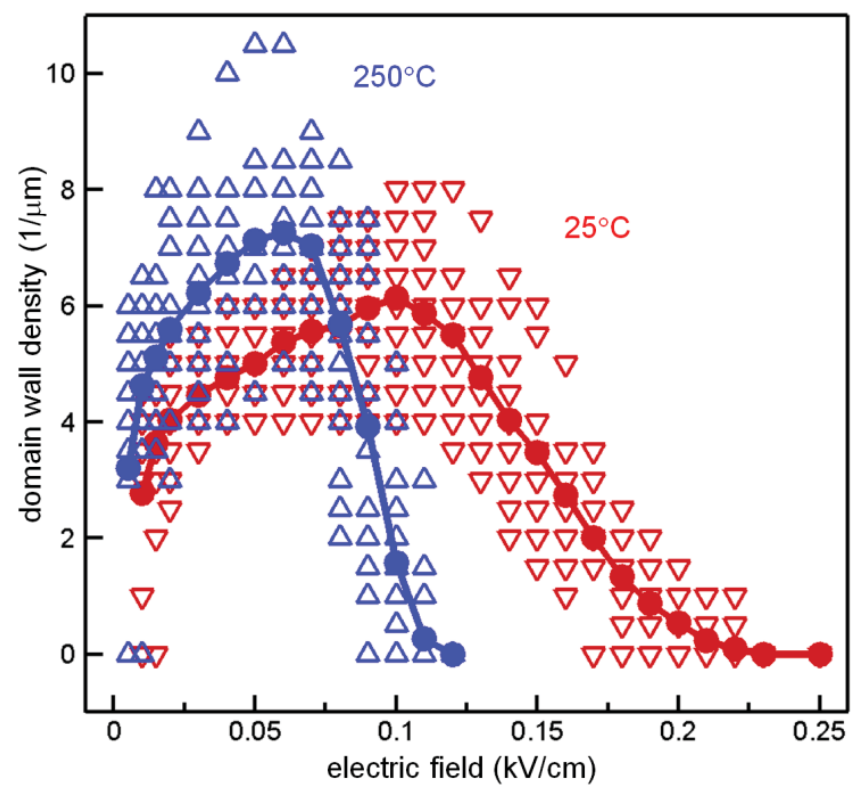

Figure 3.5 Computer simulation of dependence of domain wall density in engineered domain configuration on non-polar electric field magnitude. Open symbols represent individual simulation results. Filled symbols represent average results. Red and blue symbols correspond to quenching to $25^{\circ} \mathrm{C}$ and $250^{\circ} \mathrm{C}$, respectively.

This quantitative difference is due to greater $\left|\alpha_{1}\right|$ at lower temperature. It is worth noting that paraelectric phase loses metastability (i.e., absolutely instable) below CurieWeiss temperature $T_{0}$, thus correlated nucleation of quenched ferroelectrics exhibits isothermal growth and coarsening kinetics similar to spinodal decomposition. Because the role of interfacial energy is played by polarization gradient energy as expressed in Eq. (2.2), which is smaller at higher temperature, the domain coarsening kinetics is slower at $250^{\circ} \mathrm{C}$, resulting in higher domain wall density in final domain configurations. The smaller $\left|\alpha_{1}\right|$ at $250^{\circ} \mathrm{C}$ also flattens the free energy landscape, making easier selection of polar nuclei by external electric field thus shifting the curve in Fig. 3.5 to lower field. Except this 
quantitative difference, the same mechanism controls domain configurations and sizes as discussed above.

\subsection{Theoretical Study and Numerical Simulation for Correlated Nucleation}

Mechanisms of nucleation and growth of domains during ferroelectric phase transformation are investigated by using theoretical and computational approaches. It is shown that ferroelectric phase transformation exhibits some interesting behaviors due to strong long-range dipole-dipole-like interactions involved in the system. Incorporation of electrostatic and elastostatic energies into the classical Landau-Ginzburg-Devonshire theory effectively modifies the coefficients of the polynomial free energy function and introduces extra energy barrier for ferroelectric phase transformation. It is found that independent nucleation of ferroelectric phase in the context of classical nucleation theory is impossible, because electrostatic interaction generates an insurmountable energy barrier to isolated nucleus. Phase field modeling and computer simulation reveals that, in order to circumvent such an energy barrier, ferroelectric nucleation exhibits strong spatial correlation and self-organization behaviors from the very beginning, and ferroelectric phase transformation proceeds via spatial and temporal evolution of self-accommodating domains that provide a low-energy kinetic pathway throughout the entire phase transformation process. Theoretical analysis of the critical wavelength of correlated nucleation agrees with the computer simulation. Heterogeneous nucleation as induced by externally applied local electric field and subsequent polarization evolution process is also simulated to further demonstrate such self-organized pattern formation behaviors. 


\subsubsection{The Limitations of Classical Isolated Nucleation Theory in Ferroelectric System}

\section{Background and Classical Ferroelectric Theory Overview}

As one typical solid-state phase transformation, ferroelectric phase transformation exhibits some distinct features from other ferroic phase transformations, such as ferroelastic and ferromagnetic transformations. Unlike ferroelastic transformation that involves only spontaneous strain and elastostatic interaction, ferroelectric transformation involves both spontaneous polarization and spontaneous strain due to electrostriction. Since the typical electrostatic interaction energy density ( $e^{\text {electr }} \sim \varepsilon_{0}^{-1} P^{2}$, where $\varepsilon_{0}$ is the permittivity of free space and $P$ spontaneous polarization) is $3 \sim 4$ orders of magnitude greater than the typical elastostatic interaction energy density $\left(e^{\text {elast }} \sim E \varepsilon^{2}\right.$, where $E$ is elastic modulus and $\varepsilon$ spontaneous strain) in ferroelectrics (Using $\varepsilon_{0}=8.854 \times 10^{-12}$ $\mathrm{C}^{2} /\left(\mathrm{N} \cdot \mathrm{m}^{2}\right)$ and the values $P=0.5 \mathrm{C} / \mathrm{m}^{2}, E=50 \mathrm{GPa}$ and $\varepsilon=0.01$ typical of lead zirconate titanate, a leading piezoelectric material, it is estimated that $e^{\text {electr }} \sim 3 \times 10^{10} \mathrm{~J} / \mathrm{m}^{3}$ and $e^{\text {elast }} \sim 5 \times 10^{6} \mathrm{~J} / \mathrm{m}^{3}$ ), electrostatic interaction plays a dominant role in ferroelectric phase transformation. Similarly, ferromagnetic transformation involves both spontaneous magnetization and spontaneous strain due to magnetostriction, but the typical energy densities of magnetostatic interaction ( $e^{\text {magnet }} \sim \mu_{0} M^{2}$, where $\mu_{0}$ is the permeability of free space and $M$ spontaneous magnetization) and elastostatic interaction are usually 5 and at least 2 orders of magnitude smaller in ferromagnetics than their respective energy counterparts in ferroelectrics (Using $\mu_{0}=4 \pi \times 10^{-7} \mathrm{~N} / \mathrm{A}^{2}$ and the values $M=8 \times 10^{5} \mathrm{~A} / \mathrm{m}, E=30$ 
GPa and $\varepsilon=0.001$ typical of Terfenol-D, a leading magnetostrictive material, it is estimated that $e^{\text {magnet }} \sim 8 \times 10^{5} \mathrm{~J} / \mathrm{m}^{3}$ and $\left.e^{\text {elast }} \sim 3 \times 10^{4} \mathrm{~J} / \mathrm{m}^{3}\right)$. In fact, the electrostatic interaction in ferroelectrics is even stronger than the elastostatic interaction in shape memory alloys (Using the values $E=70 \mathrm{GPa}$ and $\varepsilon=0.1$ typical of Nitinol, a leading shape memory alloy, it is estimated that $\left.e^{\text {elast }} \sim 7 \times 10^{8} \mathrm{~J} / \mathrm{m}^{3}\right)$. Such a strong electrostatic interaction imparts some interesting behaviors to ferroelectric phase transformation. As will be shown in this section, nucleation of ferroelectric phase in the context of classical nucleation theory is impossible, because electrostatic interaction generates an insurmountable energy barrier to isolated nucleus; in order to circumvent such an energy barrier, nucleation exhibits strong spatial correlation and self-organization behaviors from the very beginning, and ferroelectric phase transformation proceeds via spatial and temporal evolution of self-accommodating domains that provide a low-energy kinetic pathway throughout the entire phase transformation process.

The free energy density of a ferroelectric system (including its paraelectric state as well) is expressed as a function of polarization vector $\mathbf{P}$, which is the thermodynamic state variable used to characterize ferroelectric phase transformation. In its conventional polynomial form, the free energy density function (2.1) is re-presented here for the referring convenience as equation (3.1):

$$
\begin{aligned}
f(\mathbf{P}) & =\alpha_{1}\left(P_{1}^{2}+P_{2}^{2}+P_{3}^{2}\right)+\alpha_{11}\left(P_{1}^{4}+P_{2}^{4}+P_{3}^{4}\right)+\alpha_{12}\left(P_{1}^{2} P_{2}^{2}+P_{2}^{2} P_{3}^{2}+P_{3}^{2} P_{1}^{2}\right) \\
& +\alpha_{111}\left(P_{1}^{6}+P_{2}^{6}+P_{3}^{6}\right)+\alpha_{112}\left[P_{1}^{4}\left(P_{2}^{2}+P_{3}^{2}\right)+P_{2}^{4}\left(P_{3}^{2}+P_{1}^{2}\right)+P_{3}^{4}\left(P_{1}^{2}+P_{2}^{2}\right)\right], \\
& +\alpha_{123} P_{1}^{2} P_{2}^{2} P_{3}^{2}
\end{aligned}
$$


where $\alpha_{i}, \alpha_{i j}$, and $\alpha_{i j k}$ are expansion coefficients that can be determined for specific ferroelectric systems by fitting the theoretical predictions to experimental data. In particular, to reproduce the Curie-Weiss law [190] of dielectric susceptibility in the paraelectric state, the coefficient of quadratic term assumes the following form: $\alpha_{1}=\frac{T-T_{0}}{2 \varepsilon_{0} C}$

where $C$ is Curie constant and $T_{0}$ Curie-Weiss temperature across which $\alpha_{1}$ changes sign from positive to negative upon cooling. The coefficients of higher-order terms are usually assumed to be temperature-independent. Depending on the signs of fourth-order term coefficients, Eq. (3.1) is able to describe both first-order and second-order transitions. For example, $\alpha_{11}>0$ and $\left(\alpha_{11}+\alpha_{12}\right) / 3>0$ correspond to second-order transition to tetragonal and rhombohedral ferroelectric phases, respectively. In second-order transition, $T_{\mathrm{C}}=T_{0}$, where $T_{\mathrm{C}}$ is Curie temperature. In first-order transition, say to tetragonal ferroelectric phase, $T_{\mathrm{C}}=T_{0}+\varepsilon_{0} C \alpha_{11}^{2} /\left(2 \alpha_{111}\right)$, which is above $T_{0}\left(\alpha_{11}<0\right.$ in first-order transition, and $\alpha_{111}>0$ to maintain ferroelectric stability, i.e., preventing $f \rightarrow-\infty$ as $P \rightarrow \infty)$. There is another characteristic temperature in first-order transition, $T_{1}=T_{0}+2 \varepsilon_{0} C \alpha_{11}^{2} /\left(3 \alpha_{111}\right)=T_{\mathrm{C}}+\varepsilon_{0} C \alpha_{11}^{2} /\left(6 \alpha_{111}\right)$, which is above $T_{\mathrm{C}}$. The three characteristic temperatures, namely, $T_{0}, T_{\mathrm{C}}$ and $T_{1}$, define four temperature ranges of different phase stabilities as summarized in Table 3.2: (i) $T>T_{1}$, stable paraelectric phase and instable ferroelectric phase; (ii) $T_{\mathrm{C}}<T<T_{1}$, stable paraelectric phase and metastable ferroelectric phase; (iii) $T_{0}<T<T_{\mathrm{C}}$, stable ferroelectric phase and metastable paraelectric phase; and (iv) $T<T_{0}$, stable ferroelectric phase and instable paraelectric phase. In 
particular, $T_{1}$ and $T_{0}$ respectively characterize the theoretical upper and lower limits of thermal hysteresis of the first-order transition. In order to thermally induce ferroelectric phase transformation upon cooling, the temperature must be lower than $T_{\mathrm{C}}$, or equivalently:

$\alpha_{1}<\alpha_{11}^{2} /\left(4 \alpha_{111}\right)$,

which is a necessary condition for ferroelectric phase transformation and will be further examined. Above is a brief discussion of ferroelectric phase transition in the context of Landau-Ginzburg-Devonshire theory in relevance to the following sections, while more comprehensive discussion can be found in classical texts such as Ref. [1].

Table 3.2 Summary of stability of different phases at various temperature ranges.

\begin{tabular}{ccc}
\hline Temperature Range & Paraelectric & Ferroelectric Phase \\
\hline$T>T_{\mathbf{1}}$ & Phase & Unstable \\
$T_{\mathrm{C}}<\boldsymbol{T}<\boldsymbol{T}_{\mathbf{1}}$ & Stable & Metastable \\
$T_{\mathbf{0}}<\boldsymbol{T}<\boldsymbol{T}_{\mathrm{C}}$ & Metastable & Stable \\
$T<T_{\mathbf{0}}$ & Unstable & Stable \\
\hline
\end{tabular}

Landau-Ginzburg-Devonshire theory, as discussed above, addresses ferroelectric phase transformation in terms of a single variable $\mathbf{P}$, i.e., dealing with a homogeneous system. The function $f(\mathbf{P})$ characterizes the non-equilibrium local bulk free energy density of a stress-free homogeneous ferroelectric state as described by $\mathbf{P}$. In reality, ferroelectric phase transformation always involves inhomogeneous states, such as nucleation and domain formation. To describe an arbitrary inhomogeneous state, position-dependent polarization vector $\mathbf{P}(\mathbf{r})$, i.e., field variable, needs to be used. The total system free energy of an inhomogeneous system is a functional of the field variable $\mathbf{P}(\mathbf{r})$ similar to equation (2.2): 
$F=\int d^{3} r\left[f(\mathbf{P})+\frac{1}{2} \beta_{i j k l} \frac{\partial P_{i}}{\partial r_{j}} \frac{\partial P_{k}}{\partial r_{l}}\right]+\frac{1}{2} \int \frac{d^{3} k}{(2 \pi)^{3}}\left[\frac{n_{i} n_{j}}{\varepsilon_{0}} \tilde{P}_{i} \tilde{P}_{j}^{*}+K_{i j k l} \tilde{\varepsilon}_{i j} \tilde{\varepsilon}_{k l}^{*}\right]$

where summation convention over repeated indices is implied. The gradient term in Eq. (3.4) characterizes the energy contribution associated with polarization change (e.g., across domain walls), where $\beta_{i j k l}$ is the gradient coefficient tensor. The k-space integral in Eq. (3.4) gives the electrostatic energy of polarization distribution $\mathbf{P}(\mathbf{r})$ and the elastostatic energy of electrostrictive strain distribution $\boldsymbol{\varepsilon}(\mathbf{r})$ (under homogeneous elastic modulus assumption) [109], where $\mathbf{n}=\mathbf{k} / k$ is a unit vector in $\mathbf{k}$-space, $K_{i j k l}=C_{i j k l}-n_{p} C_{i j p q} \Omega_{q r} C_{k l r s} n_{s}$, $C_{i j k l}$ is the elastic modulus tensor, and $\Omega_{i k}=\left(C_{i j k l} n_{j} n_{l}\right)^{-1}$ is the Green function tensor of elasticity. The functions $\tilde{\mathbf{P}}(\mathbf{k})$ and $\tilde{\boldsymbol{\varepsilon}}(\mathbf{k})$ are the Fourier transform of the fields $\mathbf{P}(\mathbf{r})$ and $\varepsilon(\mathbf{r})$, respectively, and the superscript asterisk * indicates complex conjugate. Resulting from electrostriction, the spontaneous strain $\varepsilon$ in ferroelectrics is a secondary order parameter and is coupled to the primary order parameter $\mathbf{P}$ through electrostriction coefficient tensor $Q_{i j k l}$ :

$\varepsilon_{i j}=Q_{i j k l} P_{k} P_{l}$,

Incorporation of electrostatic and elastostatic energies into the classical Landau-GinzburgDevonshire theory through Eq. (3.4) introduces extra energy barrier for ferroelectric phase transformation. In particular, electrostatic and elastostatic interactions effectively modify the coefficients of the polynomial free energy function given in Eq. (3.1). As a result, ferroelectric phase transformation behavior is significantly affected and exhibits some 
important features. This scenario in ferroelectrics is similar to the coherent structural transformations in metals and alloys [109].

\section{Theoretical Analysis of Independent Ferroelectric Nucleation: Insurmountable Energy Barrier}

In the picture of classical nucleation theory based on Gibbs' thermodynamic description of heterogeneous system [207], phase transformation proceeds via formation of nuclei of new stable phase. The free energy of the heterogeneous system is the sum of the bulk contributions of the nuclei and the untransformed metastable phase supplemented by interfacial contribution. Taking the free energy of untransformed system as reference, the energy of nucleus formation (i.e., change in system free energy during nucleation) is:

$\Delta F=V \Delta f+A \gamma$,

where $V$ is the nucleus volume, $A$ is the interfacial area, $\Delta f$ is the change of free energy density in nucleus with respect to metastable phase (thus $\Delta f<0$ ), and $\gamma$ is the interfacial free energy density (surface tension). While, in principle, the properties of the nuclei (e.g., atomic arrangement, $\Delta f$ and $\gamma$ ) are in general different from that of macroscopic stable phase, in theoretical treatment it is typically assumed that the bulk and interface properties are approximately the same in nuclei as in macrophase. For simplicity, a spherical nucleus is often considered to illustrate the essential thermodynamic feature of nucleation: bulk energy decreases with $R^{3}$ while interface energy increases with $R^{2}$, leading to a critical nucleus radius $R_{\mathrm{c}}$ :

$R_{\mathrm{c}}=-\frac{2 \gamma}{\Delta f}$ 
The system can lower its free energy by growth of supercritical nuclei (i.e., nuclei of size greater than $R_{\mathrm{c}}$ ).

Nucleation requires $\Delta f<0$. This requirement, in the case of ferroelectric phase transformation, is equivalent to the condition in Eq. (3.3) for first-order transition or $\alpha_{1}<0$ for second-order transition. However, it is worth noting that Eq. (3.6) does not consider the contributions of electrostatic and elastostatic interactions to the energy of nucleus formation. To take such interaction energies into account, the free energy functional in Eq. (3.4) must be used. In this section, a spherical polar nucleus is considered for mathematical tractability. While uniform distribution of polarization inside the nucleus is assumed, the properties of nucleus are not necessarily the same as in stable ferroelectric phase; that is, the nucleus may assume any value of $\mathbf{P}$ rather than the equilibrium spontaneous polarization of the macroscopic ferroelectric phase. Eq. (3.4) will be analytically evaluated to study the energy barrier to ferroelectric nucleation due to electrostatic and elastostatic interactions. It is noteworthy that spherical nucleus is one of a limited number of nucleus shapes that allow closed-form solutions. To fully explore all possible nucleus geometries and the energetics of nucleation, constraints on nucleus shapes have to be lifted, which makes analytical analysis mathematically intractable, thus necessitating computational approach.

For a spherical particle (nucleus) of radius $R$ and polarization $\mathbf{P}$, the first term of the k-space integral in Eq. (3.4) gives the electrostatic energy of the nucleus, as derived in Eq. (A11) in Appendix A: 
$E^{\text {electr }}=\frac{P^{2}}{6 \varepsilon_{0}} V$

or, equivalently, the electrostatic energy density of the nucleus is, as given in Eq. (A12):

$e^{\text {electr }}=\frac{P^{2}}{6 \varepsilon_{0}}$

The second term of the k-space integral in Eq. (3.4) gives the elastostatic energy of the nucleus, as derived in Eq. (A25) in Appendix. For polarization along [100] axis, such as in a nucleus of tetragonal ferroelectric phase, the elastostatic energy density of the nucleus is, as given in Eq. (A28):

$e^{\text {elast }}=\frac{E P^{4}}{15\left(1-v^{2}\right)}\left[4 Q_{11}^{2}+(9+5 v) Q_{12}^{2}+2(1+5 v) Q_{11} Q_{12}\right]$

where $E$ and $v$ are Young's modulus and Poisson's ratio, respectively, and $Q_{11}=Q_{1111}$ and $Q_{12}=Q_{1122}$ are the electrostrictive constants (i.e., the tensor components in Voigt matrix notation). For polarization along [111] axis, such as in a nucleus of rhombohedral ferroelectric phase, the elastostatic energy density of the nucleus is, as given in Eq. (A32):

$$
e^{\text {elast }}=\frac{E P^{4}}{180\left(1-v^{2}\right)}\left[20(1+v) Q_{11}^{2}+80(1+v) Q_{12}^{2}+(7-5 v) Q_{44}^{2}+80(1+v) Q_{11} Q_{12}\right]
$$

where $Q_{44}=4 Q_{1212}$. The results in Eqs. (3.8)-(3.10) are identical to the classical solutions of electrostatic and elastostatic inclusion problems [109, 208, 209].

Equations (3.8)-(3.10) show that the electrostatic and elastostatic energies can be treated as bulk energy densities of the nucleus, thus incorporation of such long-range dipole-dipole-like interactions into the Landau-Ginzburg-Devonshire theory effectively 
modifies the coefficients of the polynomial free energy function given in Eq. (3.1). For convenience, Eq. (3.1) can be rewritten in the following equivalent form:

$$
\begin{aligned}
f(\mathbf{P}) & =\alpha_{1} P^{2}+\alpha_{11} P^{4}+\left(\alpha_{12}-2 \alpha_{11}\right)\left(P_{1}^{2} P_{2}^{2}+P_{2}^{2} P_{3}^{2}+P_{3}^{2} P_{1}^{2}\right) \\
& +\alpha_{111} P^{6}+\left(\alpha_{112}-3 \alpha_{111}\right)\left[P_{1}^{4}\left(P_{2}^{2}+P_{3}^{2}\right)+P_{2}^{4}\left(P_{3}^{2}+P_{1}^{2}\right)+P_{3}^{4}\left(P_{1}^{2}+P_{2}^{2}\right)\right], \\
& +\left(\alpha_{123}-6 \alpha_{111}\right) P_{1}^{2} P_{2}^{2} P_{3}^{2}
\end{aligned}
$$

where $P=\sqrt{P_{1}^{2}+P_{2}^{2}+P_{3}^{2}}$. The effective bulk free energy density of a spherical ferroelectric nucleus is a sum of the energy contributions given in Eqs. (3.8), (3.9) [or (3.10)], and (3.11):

$$
\begin{aligned}
f_{\text {coherent }}(\mathbf{P}) & =\alpha_{1}^{\prime} P^{2}+\alpha_{11}^{\prime} P^{4}+\left(\alpha_{12}-2 \alpha_{11}\right)\left(P_{1}^{2} P_{2}^{2}+P_{2}^{2} P_{3}^{2}+P_{3}^{2} P_{1}^{2}\right) \\
& +\alpha_{111} P^{6}+\left(\alpha_{112}-3 \alpha_{111}\right)\left[P_{1}^{4}\left(P_{2}^{2}+P_{3}^{2}\right)+P_{2}^{4}\left(P_{3}^{2}+P_{1}^{2}\right)+P_{3}^{4}\left(P_{1}^{2}+P_{2}^{2}\right)\right], \\
& +\left(\alpha_{123}-6 \alpha_{111}\right) P_{1}^{2} P_{2}^{2} P_{3}^{2}
\end{aligned}
$$

where the modified coefficients are:

$$
\begin{aligned}
& \alpha_{1}^{\prime}=\alpha_{1}+\frac{1}{6 \varepsilon_{0}} \\
& \alpha_{11}^{\prime}=\alpha_{11}+\frac{E}{15\left(1-v^{2}\right)}\left[4 Q_{11}^{2}+(9+5 v) Q_{12}^{2}+2(1+5 v) Q_{11} Q_{12}\right]
\end{aligned}
$$

To be specific, elastostatic energy of a tetragonal ferroelectric nucleus, i.e., Eq. (3.9), is used in Eq. (3.13), which does not sacrifice the generality of the discussion since the most significant modification is made on $\alpha_{1}$ from electrostatic energy given in Eq. (3.8) that does not depend on the polarization direction of ferroelectric phase.

Consider the transformation from cubic paraelectric phase to tetragonal ferroelectric phase in barium titanate $\left(\mathrm{BaTiO}_{3}\right)$, a typical ferroelectric perovskite. The 
relevant coefficients of the Landau-Ginzburg-Devonshire free energy polynomial are [194]:

$\alpha_{1}=3.34 \times 10^{5}(T-381)\left(\mathrm{VmC}^{-1}\right)$

$\alpha_{11}=4.69 \times 10^{6}(T-393)-2.02 \times 10^{8} \quad\left(\mathrm{Vm}^{5} \mathrm{C}^{-3}\right)$,

$\alpha_{111}=-5.52 \times 10^{7}(T-393)+2.76 \times 10^{9} \quad\left(\mathrm{Vm}^{9} \mathrm{C}^{-5}\right)$

which gives a first-order transition at Curie temperature $T_{\mathrm{C}}=393 \mathrm{~K}$, with Curie-Weiss temperature $T_{0}=381 \mathrm{~K}$. Using the electrostrictive constants [194] $Q_{11}=0.11 \mathrm{~m}^{4} \mathrm{C}^{-2}$, $Q_{12}=-0.045 \mathrm{~m}^{4} \mathrm{C}^{-2}, Q_{44}=0.029 \mathrm{~m}^{4} \mathrm{C}^{-2}$, elastic constants $E=50 \mathrm{GPa}, v=0.3$, and the permittivity of free space $\varepsilon_{0}=8.854 \times 10^{-12} \mathrm{C}^{2} /\left(\mathrm{N} \cdot \mathrm{m}^{2}\right)$, the modified coefficients are:

$\alpha_{1}^{\prime}=3.34 \times 10^{5}(T-381)+1.88 \times 10^{10} \quad\left(\mathrm{VmC}^{-1}\right)$,

$\alpha_{11}^{\prime}=4.69 \times 10^{6}(T-393)-0.37 \times 10^{8} \quad\left(\mathrm{Vm}^{5} \mathrm{C}^{-3}\right)$.

It is observed from Eqs. (3.15a), (3.15b) and (3.14c) that, after incorporation of electrostatic and elastostatic energies, the necessary condition for ferroelectric phase transformation, as given in Eq. (3.3), cannot be satisfied within the applicability range of the theory, i.e., $\alpha_{111}>0$ (or equivalently $T<443 \mathrm{~K}$ ); that is,

$\alpha_{1}^{\prime}>\alpha_{11}^{\prime 2} /\left(4 \alpha_{111}\right)>0$

thus transition to ferroelectric tetragonal phase could not occur. The most significant modification comes from the electrostatic interaction energy that makes always $\alpha_{1}^{\prime}>0$, implying Curie-Weiss temperature would not exist. 
Above conclusion based on Eqs. (3.15) and (3.16) obviously contradicts experimental facts of ferroelectric phase transition phenomena. In fact, it just shows that electrostatic and elastostatic interactions introduce extra energy barrier for ferroelectric phase transformation, and, as a result, independent nucleation of ferroelectric phase in the context of classical nucleation theory is impossible (it is worth noting that the surface energy has even not been taken into account, which adds another energy barrier for nucleation). In particular, the electrostatic interaction generates an insurmountable energy barrier to isolated ferroelectric nucleus. In order to circumvent such an energy barrier, ferroelectric nucleation exhibits strong spatial correlation and self-organization behaviors from the very beginning of the transformation, as revealed by the computer simulations presented in next section. It is worth noting that above discussion generally holds also for other ferroelectric systems, such as lead zirconate titanate $\left[\mathrm{Pb}\left(\mathrm{Zr}_{1-x} \mathrm{Ti}_{x}\right) \mathrm{O}_{3}(\mathrm{PZT})\right][195]$. As a matter of fact, the electrostatic interaction energy, which is orders of magnitude greater than $\alpha_{1}$, makes condition similar to Eq. (3.16) always hold for transition to any ferroelectric phase (tetragonal, rhombohedral, or orthorhombic) irrespective of the order of the transition (first or second).

\subsubsection{Correlated Nucleation Process and Domain Formation}

\section{Computer Simulation of Correlated Nucleation: Self-Organization Behavior}

In the phase field modeling, the thermodynamics (i.e., total free energy) of a ferroelectric system is formulated in Eq. (3.4), and the kinetics (i.e., polarization evolution) 
is characterized by the time-dependent Ginzburg-Landau equation adopted from equation (2.4):

$\frac{\partial \mathbf{P}(\mathbf{r}, t)}{\partial t}=-L \frac{\delta F}{\delta \mathbf{P}(\mathbf{r}, t)}+\xi(\mathbf{r}, t)$,

where $L$ is the kinetic coefficient, and $\xi(\mathbf{r}, t)$ is Gaussian-distributed Langevin noise term that accounts for the effect of thermal fluctuation. Note that, as a secondary order parameter coupled to the primary order parameter $\mathbf{P}(\mathbf{r}, t)$ through Eq. (3.5), electrostrictive strain $\boldsymbol{\varepsilon}(\mathbf{r}, t)$ is not an independently evolving field variable. In computer simulations, the experimentally determined material parameters specific to $\mathrm{Pb}\left(\mathrm{Zr}_{0.4} \mathrm{Ti}_{0.6}\right) \mathrm{O}_{3}$ are used, which describe a first-order transition to ferroelectric tetragonal phase. An isotropic gradient coefficient tensor $\beta_{i j k l}=\beta \delta_{i k} \delta_{j l}$ with $\beta=\beta^{*}\left|\alpha_{1}\right| l^{2}$ is used, where $\beta^{*}=5$ is reduced dimensionless gradient coefficient and $l=2 \mathrm{~nm}$ is computational mesh size. Periodic boundary condition is employed to evaluate the Fourier transform $\tilde{\mathbf{P}}(\mathbf{k})$ and $\tilde{\boldsymbol{\varepsilon}}(\mathbf{k})$ appearing in Eq. (3.4) by using parallel fast Fourier transform algorithm.

In the computer simulation, $\mathrm{Pb}\left(\mathrm{Zr}_{0.4} \mathrm{Ti}_{0.6}\right) \mathrm{O}_{3}$ is quenched from paraelectric state to room temperature, and Eq. (3.17) is numerically solved to capture the detailed evolution process of polarization field $\mathbf{P}(\mathbf{r}, t)$. Figs. 3.6-3.9 show the polarization evolution and pattern formation during the entire ferroelectric phase transformation process from nucleation to fully developed domains. 


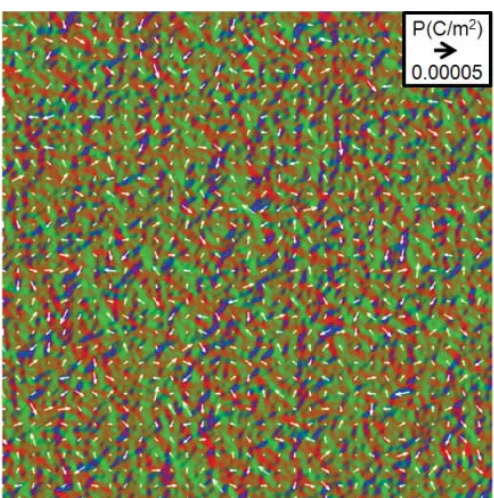

(a) $t^{\star}=1$

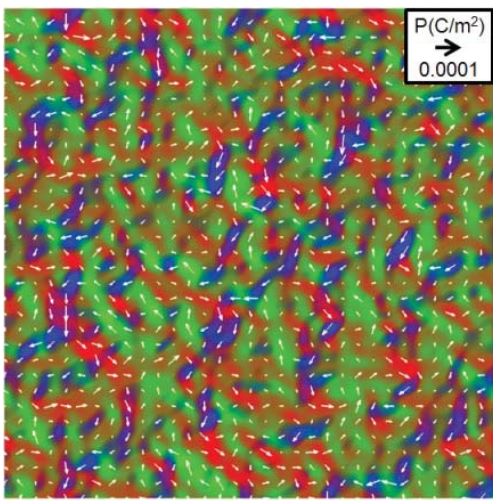

(c) $t^{*}=10$

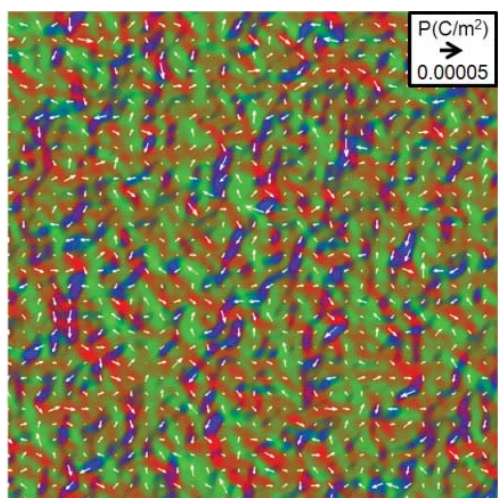

(b) $t^{*}=5$

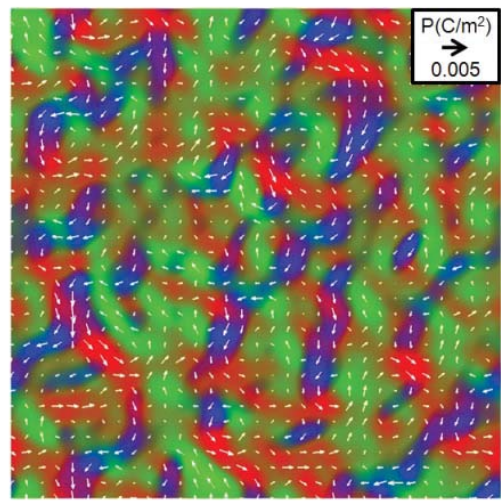

(d) $t^{*}=32$

Figure 3.6 Computer simulation of polarization evolution and pattern formation during early stage of ferroelectric phase transformation. Patterns are presented in colors with red, green, blue (RGB) components corresponding to polarization components thus representing polarization directions. White arrows represent the in-plane components of polarization vector. Black arrow in legend shows polarization magnitude. An important feature of early-stage transformation is that vortex structures remain isotropic during coarsening and polarization growth.

Based on distinct features of self-organization behaviors, the transformation process can be discussed in three stages, namely, early, intermediate and late stages as shown in Figs. 3.6, 3.8 and 3.9, respectively. The early stage of ferroelectric phase transformation is shown in Fig. 3.6, where the magnitude of transient polarization is very small as compared to the equilibrium spontaneous polarization in fully developed domains 
$\left(0.6 \mathrm{C} / \mathrm{m}^{2}\right.$ at $\left.25^{\circ} \mathrm{C}\right)$ shown in Fig. 3.9(d). Due to the smallness of polarization, elastostatic interaction is insignificant in the early stage, since $e^{\text {elast }} \propto \varepsilon^{2} \propto P^{4}$. Nevertheless, electrostatic interaction plays a significant role, as shown in Eqs. (3.15a) and (3.16) and discussed previously, which prevents independent nucleation events. Instead, polarization vectors exhibit strong spatial correlations and form vortex structures from the very beginning of transformation as shown in Fig. 3.6(a). An enlarged portion of Fig. 3.6(a) is presented in Fig. 3.7 to show the details of the polarization vortex structure, which effectively eliminates local volume charges, i.e., $\rho=-\nabla \cdot \mathbf{P}(\mathbf{r})=0$ where $\rho$ is volume charge density, thus helping circumvent the electrostatic energy barriers to nucleation. For small polarization magnitude during early stage of transformation, the isotropic quadratic term of the Landau-Ginzburg-Devonshire polynomial energy function in Eq. (3.1) dominates over anisotropic higher-order terms, which leads to isotropic vortex structures as shown in Fig. 3.6, where polarization exhibits no preferred orientation. Such selforganization behaviors persist during the gradual growth of polarization magnitude and coarsening of vortex structures, as shown in Figs. 3.6(b)-(d). An important feature of the early-stage transformation is that the vortex structures remain isotropic during coarsening and polarization growth. The detailed kinetics of polarization growth and pattern coarsening can be understood from wavelength analysis as presented later. 


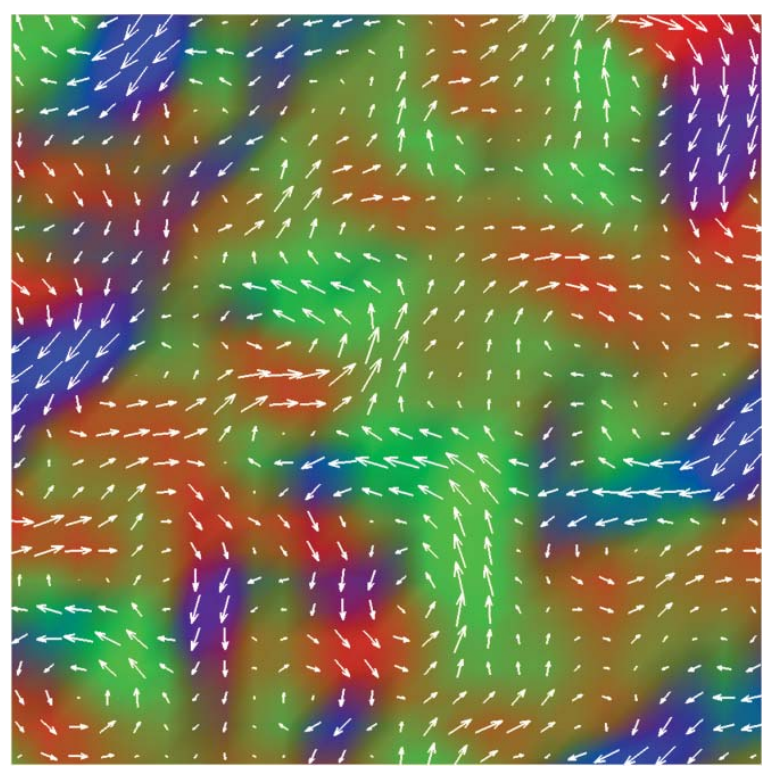

Figure 3.7 Enlarged (10×) portion of Fig. 3.6(a) to show the details of polarization vortex structures formed by correlated nucleation during early-stage ferroelectric phase transformation.

Fig. 3.8 shows the intermediate stage of ferroelectric phase transformation. Polarization magnitude continues to grow, and vortex structures continue to coarsen, as shown in Figs. 3.8(a)-(c). During the same time, due to the bigger polarization magnitude, the anisotropic higher-order terms of the Landau-Ginzburg-Devonshire polynomial energy function in Eq. (3.1) start to play a role, leading to preferred orientations of polarization along $\langle 100\rangle$ and $<111\rangle$ axes, which are the respective polar axes of stable tetragonal and metastable rhombohedral ferroelectric phases, as shown in Fig. 3.8(c). As a result of the alignment of local polarization along these polar axes, self-accommodating head-to-tail domain structures start to form, as shown in Fig. 3.8(d), where charge-free domain walls are formed along specific orientations such that $\sigma=\Delta \mathbf{P} \cdot \mathbf{n}=0$ where $\sigma$ is interface charge density, $\Delta \mathbf{P}$ is polarization change across the interface, and $\mathbf{n}$ is the interface normal vector. 
It is worth noting that such charge-accommodating domain structures minimize electrostatic interaction energy, but do not eliminate electrostatic interaction energy that is caused by electrostriction. Due to the increased polarization magnitude, electrostrictive strain also increases and starts to play a role. As a result, twin boundaries are formed between domains of the same phase (tetragonal or rhombohedral) to reduce electrostatic energy; nevertheless, stable tetragonal and metastable rhombohedral domains coexist to form bridging domain structures, where the inter-phase domain boundaries are not twin boundaries thus cannot accommodate misfit strain. As has been shown in previous study [202], such a bridging domain mechanism reduces the sum of electrostatic, elastostatic and polarization gradient energies in complex domain structures, which arise from the frustrations caused by the crystal lattice misfit among multiple structural orientation variants and the polarization distribution among multiple polar axes, thus providing a lowenergy kinetic pathway for ferroelectric phase transformation to fully self-accommodating domain structures achieved at late stage of the transformation. An important feature of the intermediate-stage transformation is that the isotropic vortex structures gradually transform into charge-accommodating bridging domain structures with preferred polarization orientations defined by the electrocrystalline anisotropy. 


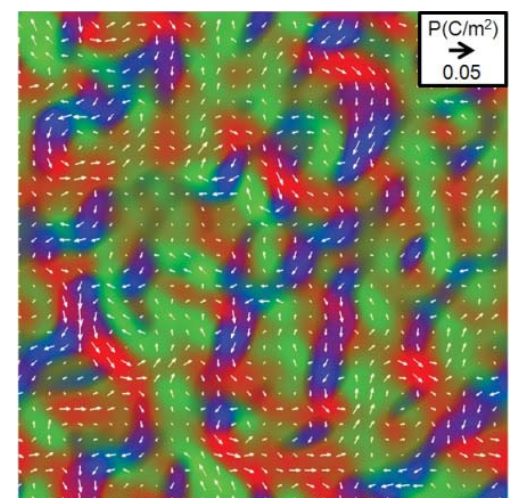

(a) $t^{*}=45$

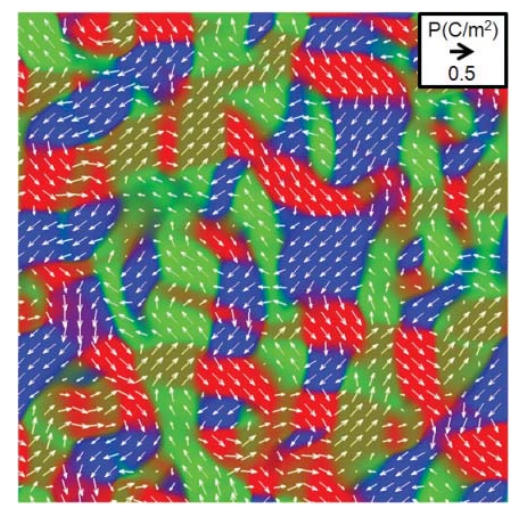

(c) $t^{*}=70$

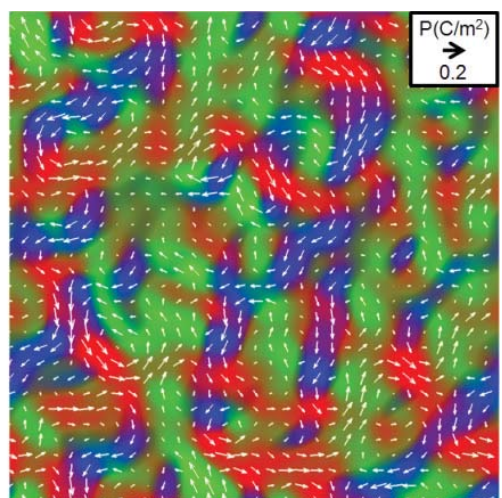

(b) $t^{*}=55$

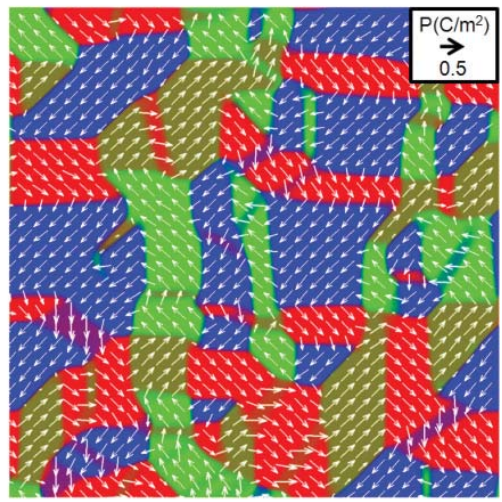

(d) $t^{*}=160$

Figure 3.8 Computer simulation of polarization evolution and pattern formation during intermediate stage of ferroelectric phase transformation. Patterns are presented in colors with red, green, blue (RGB) components corresponding to polarization components thus representing polarization directions. White arrows represent the in-plane components of polarization vector. Black arrow in legend shows polarization magnitude. An important feature of intermediate-stage transformation is that isotropic vortex structures gradually transform into charge-accommodating bridging domain structures with preferred polarization orientations. 


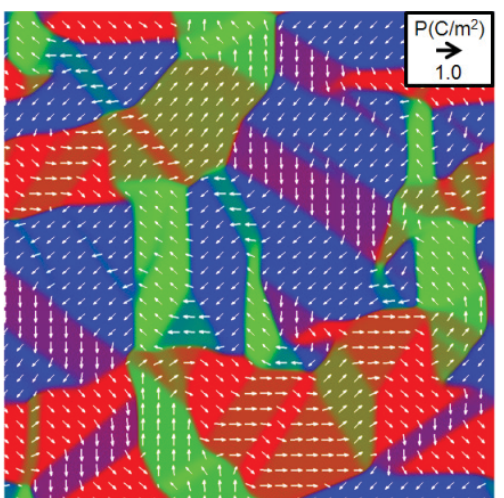

(a) $t^{*}=350$

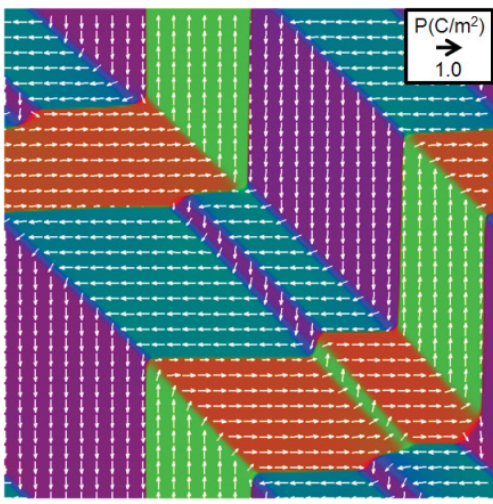

(c) $t^{*}=600$

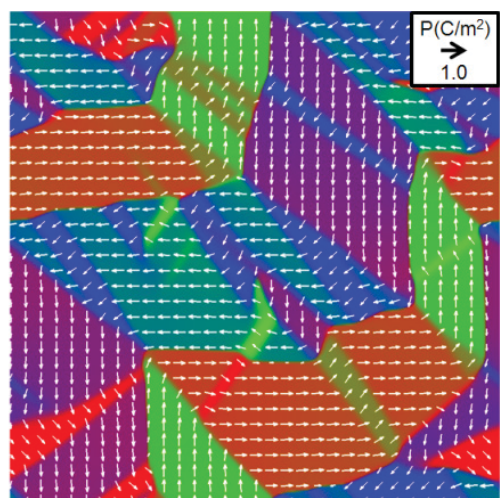

(b) $t^{*}=450$

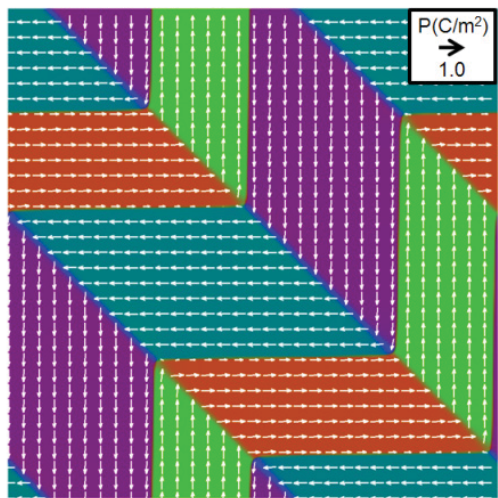

(d) $t^{*}=1200$

Figure 3.9 Computer simulation of polarization evolution and pattern formation during late stage of ferroelectric phase transformation. Patterns are presented in colors with red, green, blue (RGB) components corresponding to polarization components thus representing polarization directions. White arrows represent the in-plane components of polarization vector. Black arrow in legend shows polarization magnitude. An important feature of latestage transformation is that charge-accommodating bridging domain structures develop into fully self-accommodating twinned domain structures with head-to-tail polarization patterns that simultaneous minimize both electrostatic and elastostatic energies.

Fig. 3.9 shows the late stage of ferroelectric phase transformation. Elastostatic interaction dominates the domain evolution at this stage. In particular, the elastostatic energy associated with the inter-phase domain boundaries is reduced by gradual shrinking and final disappearance of bridging domains of metastable rhombohedral phase, as shown 
in Figs. 3.9(a)-(c). The fully developed domain structure, as shown in Fig. 3.9(d), consists of stable tetragonal phase in twin-related multi-domain state with head-to-tail polarization patterns that simultaneous minimize both electrostatic and elastostatic energies: all domain walls $\left(180^{\circ}\right.$ and $\left.90^{\circ}\right)$ are free of charge, and $90^{\circ}$ domain walls are also twin boundaries to accommodate electrostrictive strain. An important feature of the late-stage transformation is that the charge-accommodating bridging domain structures gradually develop into fully self-accommodating twinned domain structures with head-to-tail polarization patterns.

\section{Critical Wavelength of Correlated Nucleation}

The kinetics of correlated nucleation and subsequent growth and coarsening during the early stage of ferroelectric phase transformation can be understood from wavelength analysis. For very small polarization magnitude as shown in Fig. 3.6, only the first term of

the Landau-Ginzburg-Devonshire polynomial energy function in Eq. (3.1) plays a significant role while higher-order terms are negligible, reducing Eq. (3.1) to a quadratic form:

$f(\mathbf{P}) \sim \frac{T-T_{0}}{2 \varepsilon_{0} C} P^{2}$,

where Eq. (3.2) has been used. During correlated nucleation, the electrostatic interaction energy is eliminated through vortex formation, and the elastostatic interaction energy is negligibly small due to the smallness of polarization, therefore the total system free energy in Eq. (3.4) becomes effectively:

$$
F \sim \int d^{3} r\left[\frac{T-T_{0}}{2 \varepsilon_{0} C} P^{2}+\frac{1}{2} \beta \frac{\partial P_{i}}{\partial r_{j}} \frac{\partial P_{i}}{\partial r_{j}}\right],
$$


where isotropic gradient coefficient tensor is used. The kinetic equation (3.17) can be written explicitly as:

$\frac{\partial P_{i}(\mathbf{r}, t)}{\partial t}=-L\left[\frac{T-T_{0}}{\varepsilon_{0} C} P_{i}(\mathbf{r}, t)-\beta \nabla^{2} P_{i}(\mathbf{r}, t)\right]$,

which, upon Fourier transform, becomes:

$\frac{\partial \tilde{P}_{i}(\mathbf{k}, t)}{\partial t}=L\left[\frac{T_{0}-T}{\varepsilon_{0} C}-\beta k^{2}\right] \tilde{P}_{i}(\mathbf{k}, t)$,

whose solution is of the following form:

$\tilde{\mathbf{P}}(\mathbf{k}, t)=\tilde{\mathbf{P}}(\mathbf{k}, 0) e^{\kappa t}$,

where $\tilde{\mathbf{P}}(\mathbf{k}, 0)$ is initial value, and

$\kappa=L\left(\frac{T_{0}-T}{\varepsilon_{0} C}-\beta k^{2}\right)$

is exponential growth/decay constant depending on its sign. In order to nucleate, a positive (growth) exponential constant is required, i.e., $\kappa>0$, yielding (the kinetic coefficient $L>0$ )

$\frac{T_{0}-T}{\varepsilon_{0} C}>\beta k^{2}$,

which gives

$k<k_{c}=\sqrt{\frac{T_{0}-T}{\beta \varepsilon_{0} C}}$,

where $k_{c}$ is the critical wavenumber. Using $k=2 \pi / \lambda$, Eq. (3.25) gives

$\lambda>\lambda_{c}=2 \pi \sqrt{\frac{\beta \varepsilon_{0} C}{T_{0}-T}}$, 
where $\lambda_{c}$ is the critical wavelength. It shows that correlated nucleation must exhibit spatial patterns of wavelengths longer than the critical wavelength $\lambda_{c}$ in order to grow in polarization magnitude. It is worth noting that the results of critical wavelength analysis in Eqs. (3.25) and (3.26) are valid only for the early-stage transformation where polarization magnitude is small. With increased polarization magnitude, higher-order terms in the Landau-Ginzburg-Devonshire polynomial energy function and electrostatic and elastostatic interaction energies must be taken into account, making analytical treatment intractable thus necessitating numerical solutions as in above computer simulations.

Fig. 3.10 shows the wavelength-dependent polarization evolution during the earlystage ferroelectric phase transformation at various simulation times corresponding to the polarization patterns in Fig. 3.6(a)-(d). The spectral distributions are calculated by fast Fourier transform of the polarization fields shown in Fig. 3.6. The simulation starts from a random initial field of polarization vectors, which contains all wavelength components of roughly equal magnitudes as shown by the black curve $\left(t^{*}=0\right)$ in Fig. 3.10. Because the initial random polarization distribution does not form vortex structures characteristic of correlated nucleation, electrostatic interaction is significant, resulting in an initial decay of polarization at all wavelength, as shown in the purple curve $\left(t^{*}=1\right)$ in Fig. 3.10; nevertheless, polarization rapidly forms vortex structures to circumvent electrostatic interaction energy barriers, as shown in Figs. 3.6(a) and 3.7. Upon correlated nucleation, polarization subsequently evolves according to the exponential law in Eq. (3.22). In particular, the spectral components of wavelength $\lambda<\lambda_{c}$ decay, and the components of longer wavelength $\lambda>\lambda_{c}$ grow, as shown by the blue $\left(t^{*}=5\right)$, green $\left(t^{*}=10\right)$ and red $\left(t^{*}=32\right)$ 
curves in Fig. 3.10. To compare with the analytical result in Eq. (3.25), the critical wavenumber $k_{c}$ is plotted as dashed lines in Fig. 3.10, highlighting the growth zone in wavenumber (wavelength). Substituting $\beta=\beta^{*}\left|\alpha_{1}\right| l^{2}$ and $\beta^{*}=5$ into Eq. (3.25) yields $k_{c}=0.1 \times 2 \pi / l$, in agreement with the computer simulation.

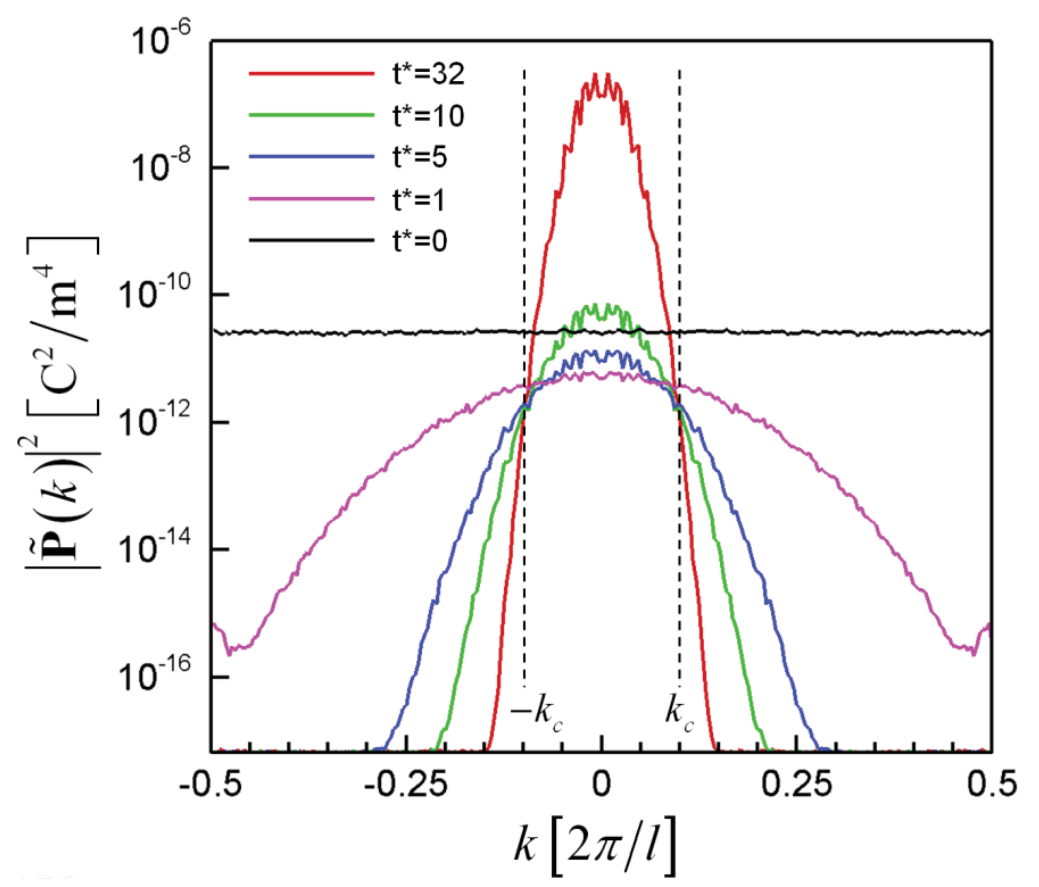

Figure 3.10 Computer simulation of wavelength (wavenumber) dependence of polarization evolution during early stage of ferroelectric phase transformation. Spectral distributions are calculated by fast Fourier transform of the polarization fields in Fig. 3.6(a)-(d). Dashed lines indicate growth zone $k<k_{c}$. 


\subsubsection{Induced Heterogeneous Nucleation to Design Stripe Domain}

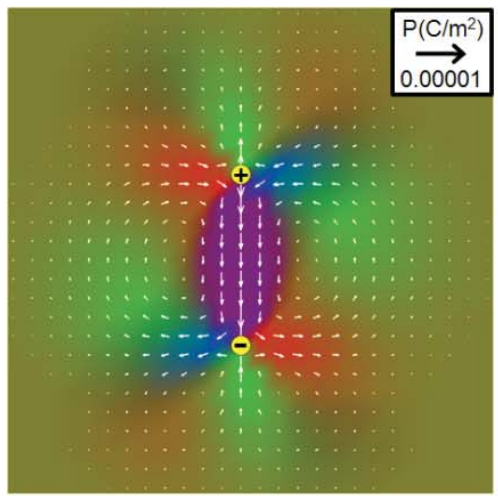

(a) $t^{*}=15[20 \times]$

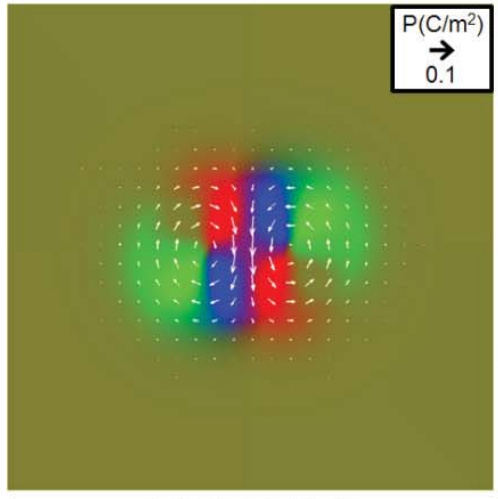

(d) $t^{*}=90[5 \times]$

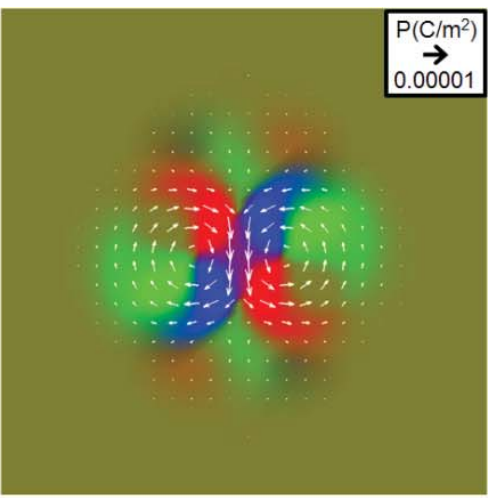

(b) $t^{*}=30[10 \times]$

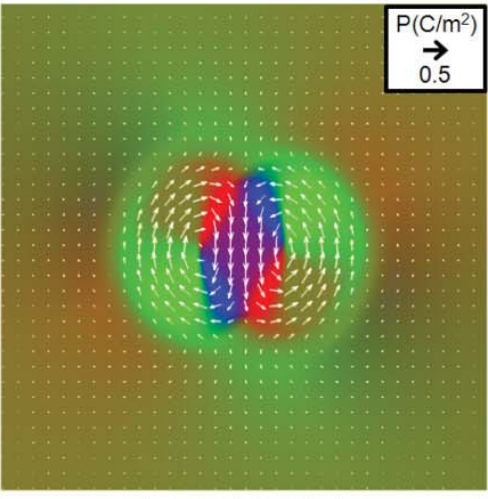

(e) $t^{*}=110[4 \times]$

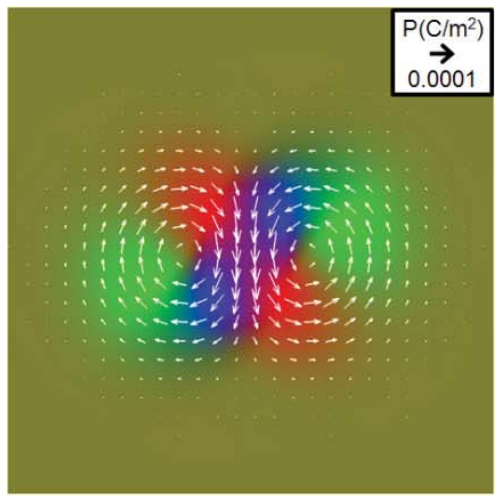

(c) $t^{*}=50[10 \times]$

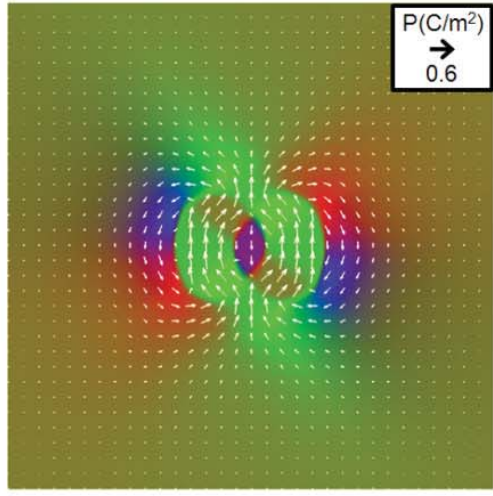

(f) $t^{*}=125[2 \times]$

Figure 3.11 Computer simulation of polarization evolution and pattern formation during early stage of ferroelectric phase transformation via local field-induced heterogeneous nucleation. Patterns are presented in colors with red, green, blue (RGB) components corresponding to polarization components thus representing polarization directions. White arrows represent the in-plane components of polarization vector. Black arrow in legend shows polarization magnitude. Enlarged portion of the simulation system is shown to reveal the details of polarization evolution, with magnification indicated below each panel. The external charge dipole generating local electric field is illustrated in (a).

In previous section, homogeneous nucleation is considered. In this section, heterogeneous nucleation as induced by externally applied local electric field is considered. The local electric field is generated by a pair of positive and negative point charges 
separated by a distance $9 l$, as illustrated in Fig. 3.11(a), which mimics the effect of the tips of external electrodes placed in the immediate vicinity of sample surface. A weak local electric field is used to induce heterogeneous nucleation, with a magnitude of $3 \mathrm{~V} / \mathrm{cm}$ at the midpoint between the two point charges. The system is quenched to room temperature with noise term in Eq. (3.17) switched off to reveal the sole effect of local field. Fig. 3.11 shows the polarization evolution during locally induced heterogeneous nucleation and early stage of the subsequent pattern formation process. As shown in Fig. 3.11(a), the region between the point charges is polarized in $-y$ direction (i.e., from positive charge to negative charge), and the regions above the positive charge and below the negative charge are polarized in $+y$ direction, as expected from the local electric field directions of an electric dipole. However, the polarization vectors in other regions around the charge dipole do not follow the local electric field directions of an electric dipole. In particular, the dipole-generated electric field diverges from the positive charge and converges into the negative charge, and is in $-y$ direction in the regions parallel to the dipole (i.e., to the left and right); in contrast to such expectations, polarization vectors are just in opposite directions to the dipole local field except in the regions between, above and below the charge dipole, as shown in Fig. 3.11(a). This behavior is a direct reflection of correlated nucleation and self-organization through electrostatic interactions: once non-uniformly polarized, the polarization field generates local charge distribution $\rho=-\nabla \cdot \mathbf{P}(\mathbf{r})$ in addition to the external point charges, and it is the total electric field that determines the polarization evolution during heterogeneous nucleation. As a result, polarization forms vortex structures around the charge dipole, as in the case of homogeneous nucleation 
shown in Figs. 3.6(a) and 3.7. Because of the smallness of the point charges, polarization of very small magnitude in Fig. 3.11(a) is able to generate local charge sufficient to compete with the external charge, thus revealing the self-organization behaviors (otherwise, large amount of external charges will dominate nucleation and growth process, as in the case of field-cooling). With growing polarization magnitude, the effect of small amount of external charges become increasingly insignificant, and the polarization vortex structures grow round (i.e., becoming isotropic) due to the still-small polarization, as shown in Figs. 3.11(b) and (c) and discussed for the early stage of homogeneous nucleation shown in Fig. 3.6. With further growing polarization magnitude, electrostatic interaction becomes stronger, and vortex structures grow in size and local polarization vectors start to align along preferred directions as a result of electrocrystalline anisotropy, as shown in Figs. 3.11(d) and (e). In particular, the strong electrostatic interaction polarizes the outer regions to the opposite directions to form layers surrounding the original heterogeneous nucleus core region, as shown in Fig. 3.11(f), leading to more complex domain evolution process as shown in Fig. 3.12.

Fig. 3.12 shows the polarization evolution and domain pattern formation subsequent to local field-induced heterogeneous nucleation. With strong electrostatic and elastostatic interactions, bridging domain microstructures of coexisting tetragonal and rhombohedral phases are formed throughout the entire system, which provide low-energy kinetic pathways to the final stripe domain structure of stable tetragonal phase. It is interesting to notice that the original core region of heterogeneous nucleation undergoes multiple polarization switching processes, from original $-y$ direction shown in Fig. 3.11, 
to $+y$ direction shown in Figs. 3.12(a)-(c), to $\pm x$ directions shown in Figs. 3.12(d) and (e), and finally to $-y$ direction again shown in Fig. 3.12(f). Such complex domain evolution processes are results of the self-organization behaviors to simultaneously minimize the microstructure-dependent electrostatic and elastostatic interaction energies.

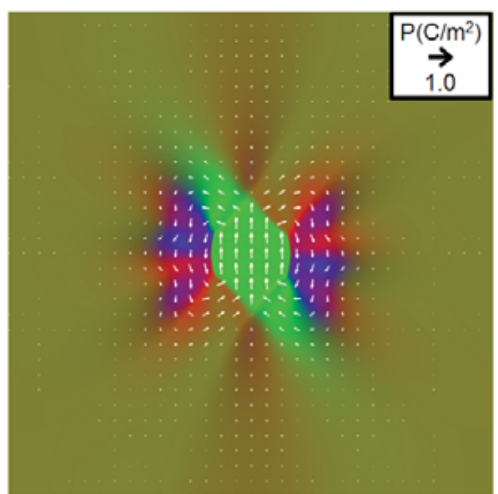

(a) $t^{*}=140$

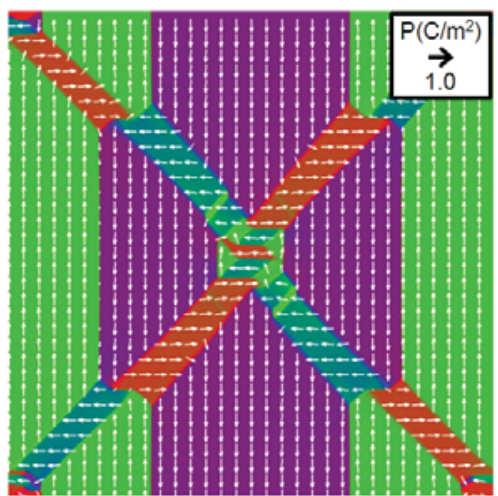

(d) $t^{*}=320$

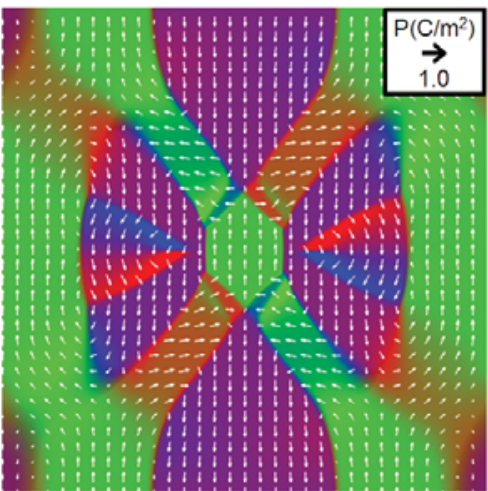

(b) $t^{*}=160$

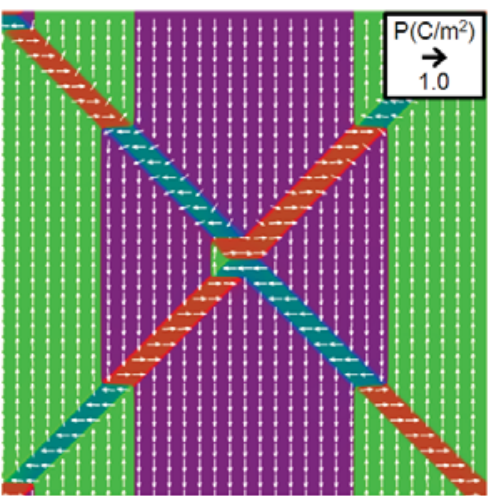

(e) $t^{\star}=800$

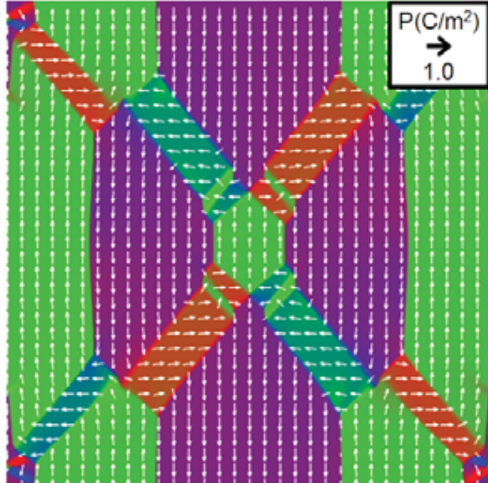

(c) $t^{*}=200$

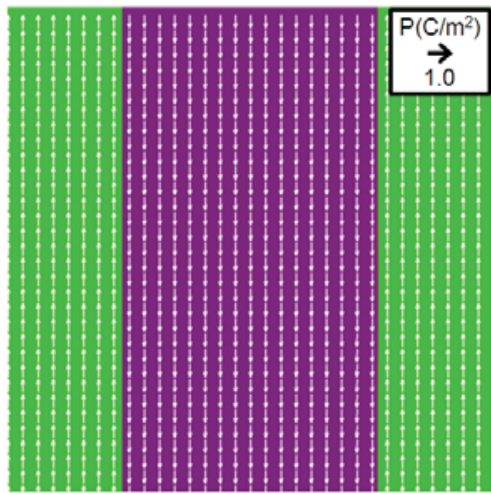

(f) $t^{\star}=2600$

Figure 3.12 Computer simulation of polarization evolution and pattern formation subsequent to local field-induced heterogeneous nucleation. Patterns are presented in colors with red, green, blue (RGB) components corresponding to polarization components thus representing polarization directions. White arrows represent the in-plane components of polarization vector. Black arrow in legend shows polarization magnitude. 


\subsection{Conclusion}

Computer simulation shows that application of electric field along non-polar axes is an effective way to control domain microstructures and wall densities in ferroelectric single crystals. It is found that selection of polar variant nuclei by non-polar electric field significantly affects subsequent domain growth and evolution kinetics and is the mechanism controlling final domain configurations and sizes, indicating that appropriate combination of electric field magnitude, direction and temperature can provide optimal processing conditions for crystallographic domain engineering.

Ferroelectric phase transformation is shown to exhibit strong correlation and selforganization behaviors during the entire transformation process from nucleation to final domain formation. Electrostatic interaction plays a significant role from the very early stage of transformation, while elastostatic interaction becomes increasingly important with growing polarization magnitude during later stage. In either homogeneous or heterogeneous nucleation, ferroelectric phase transformation starts with correlated nucleation with polarization forming vortex structures to circumvent electrostatic energy barrier. Such vortex structures are isotropic at small polarization and become anisotropic at increased polarization due to electrocrystalline anisotropy, resulting in bridging domain structures of coexisting stable and metastable ferroelectric phase, which provide lowenergy kinetic pathways to the final domain structures of stable phase. Better understanding of ferroelectric nucleation mechanisms supplies a complementary way to conduct domain pattern design. 


\section{Chapter 4. Grain Texturing by Templated Grain Growth}

While the properties of individual grains in polycrystals are generally anisotropic, untextured polycrystalline materials with random grain orientations exhibit isotropic macroscopic properties that are independent of directions. To exploit the intrinsic anisotropic properties, single crystals discussed previously are desired, which, however, are usually costly or impossible to fabricate. In order to obtain anisotropic properties in polycrystalline materials, crystallographic texturing of grains is required [210]. Texture refers to the collective distribution of grain orientations towards preferred orientation in a polycrystal. To achieve a preferred (non-random) distribution of the crystallographic orientations of grains (i.e., texture), special processing routes are needed. For example, uniaxial (or fiber) texture can be obtained through templated grain growth (TGG) in tape casted green forms [38]. Such processing technique and resultant uniaxial texture are particularly important for achieving enhanced electromechanical properties of piezoelectric ceramics. Single crystals of perovskite-type pseudo-binary ferroelectric solid solutions with compositions near morphotropic phase boundaries, such as $\mathrm{Pb}\left[\left(\mathrm{Mg}_{1 / 3}\right.\right.$ $\left.\left.\mathrm{Nb}_{2 / 3}\right)_{1-\mathrm{x}} \mathrm{Ti}_{\mathrm{x}}\right] \mathrm{O}_{3}$ (PMN-PT) and $\mathrm{Pb}\left[\left(\mathrm{Zn}_{1 / 3} \mathrm{Nb}_{2 / 3}\right)_{1-\mathrm{x}} \mathrm{Ti}_{\mathrm{x}}\right] \mathrm{O}_{3}$ (PZN-PT), exhibit optimum piezoelectric properties along [001] axis [62]. Due to the high cost of single crystal growth, [001]-textured polycrystalline ceramics of PMN-PT and PZN-PT offer better cost-

$¥$ Part of contents in this chapter was previously published in EPD Congress 2014, John Wiley \& Sons, Inc., pages 299-307. Refer Appendix E for granted permission to be republished. 
performance ratio, potentially achieving piezoelectric properties close to that of single crystals but at much lower cost [125]. Such textured ceramics are even more critical for lead zirconate titanate, $\mathrm{Pb}\left(\mathrm{Zr}_{1-\mathrm{x}} \mathrm{Ti}_{\mathrm{x}}\right) \mathrm{O}_{3}(\mathrm{PZT})$, which is currently the most widely used piezoelectric material but is intractable for single crystal growth [14]. Therefore, development of uniaxial texture in ferroelectric polycrystalline ceramics through advanced processing, such as TGG, is of technological importance for applications of piezoelectric ceramics in sensors, actuators and transducers.

In this Chapter, TGG process and grain texture development in ferroelectric polycrystalline ceramics are studied by phase field simulation and complementary computational X-ray diffraction. Phase field model is employed to simulate the polycrystalline grain structure evolution during TGG. X-ray diffraction peak intensities of the simulated polycrystals are computed during grain structure evolution. Texture development is characterized by the evolution of Lotgering factor. In particular, the effects of template seed volume fraction and sizes on the final grain structure and texture are investigated. The quantitative details of grain structure evolution and texture development gained from the computations help better understand the TGG technique and improve material processing to achieve better piezoelectric properties in textured ceramics. In order to validate our model, relative experiments are performed by our collaborators at Virginia Tech. They conducted experimental measurements to characterize the grain structures and textures at different sintering stages to make comparison with our simulations. 


\subsection{Phase Field Simulation of Templated Grain Growth}

To simulate TGG process, we employ the phase field model of grain growth presented in section 2.2 by implementing Eqs. (2.5)-(2.7). Taking into consideration of some special features of TGG associated with the templates as already stated in section 2.2, Eq. (2.7) is solved only for matrix grains to describe their growth and coarsening processes, while the long-range order parameter fields for template seeds are not evolved, which characterize the "inert" template grains during TGG. With this minor modification, the new formulated phase field model of grain growth is employed to simulate the grain structure evolutions in polycrystals during TGG process.

Figs. 4.1, 4.2 and 4.3 show the simulated processes of evolving grain structures during grain growth in polycrystals with $0 \%, 0.63 \%$ and $2.37 \%$ volume fractions of templates, respectively. Grain structures are presented as function of reduced simulation time $t^{*}$. In particular, with periodic boundary condition for the simulation box, none, one and three template seeds are respectively used to control the template volume fraction. It is observed that the big grains with the same orientation of the templates grow at the expense of other smaller matrix grains via coarsening process, gradually converting the matrix volume into the well-controlled template orientation thus increasing the degree of texture in the polycrystals. It is also observed that the reoriented big grains grow rapidly to impinge onto each other, while the randomly oriented smaller matrix grains grow much slower and are retained in the gaps between oriented big grains. It is worth noting that the heterogeneous nucleation of matrix phase on the template surface is a phenomenon of different time and length scales, which is not treated in the mesoscale phase field model of 
grain growth; instead, the simulation starts from the big grains formed from the neighboring matrix grains in direct contact with the template seeds due to their heterogeneous nucleation and epitaxial growth on the templates to achieve common orientation, as experimentally observed $[\underline{38}, \underline{125}]$ and, in particular, shown in Fig. 4.10(a)(c) in Section 4.3.

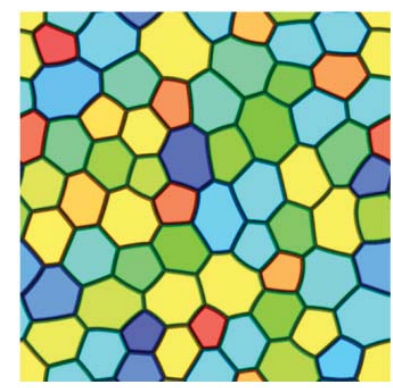

(a) $t^{\star}=25$

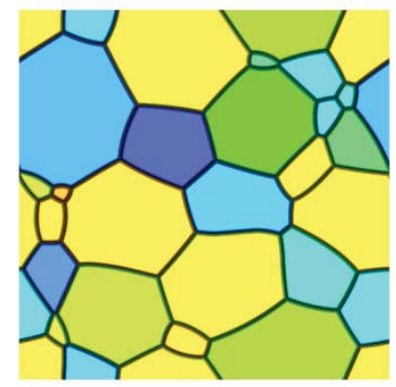

(d) $t^{*}=325$

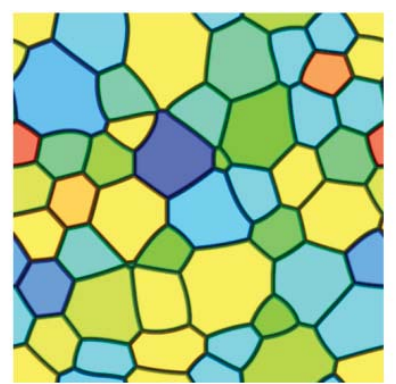

(b) $t^{\star}=125$

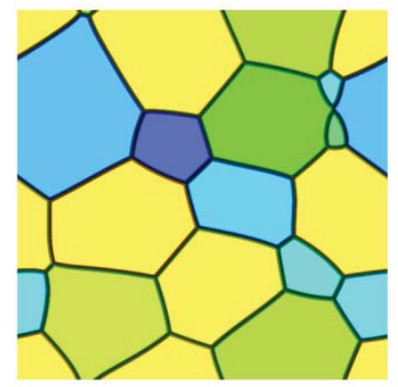

(e) $t^{*}=425$

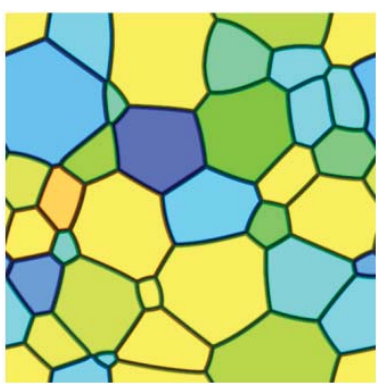

(c) $t^{*}=225$

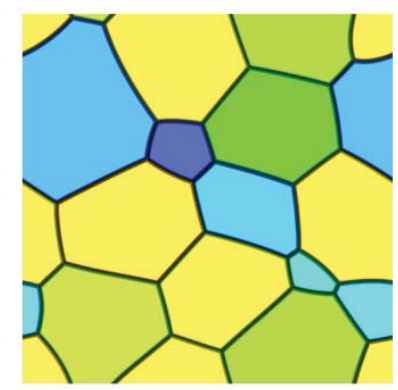

(f) $t^{*}=500$

Figure 4.1 Simulated process of grain growth without template seed [211]. (Refer Appendix $\mathrm{E}$ for permission to republish the figure) 


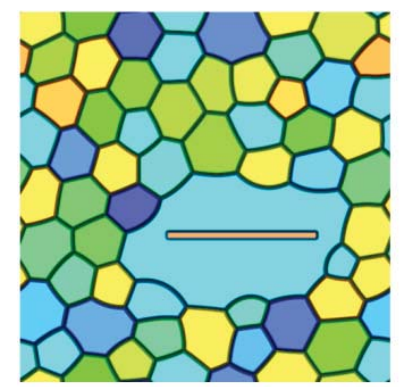

(a) $t^{*}=25$

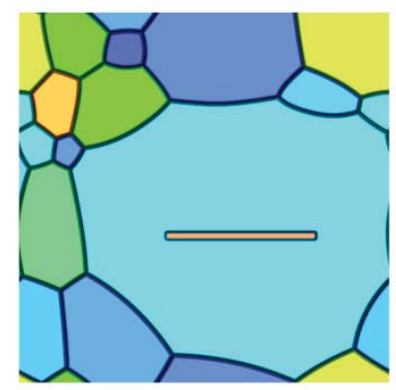

(d) $t^{*}=325$

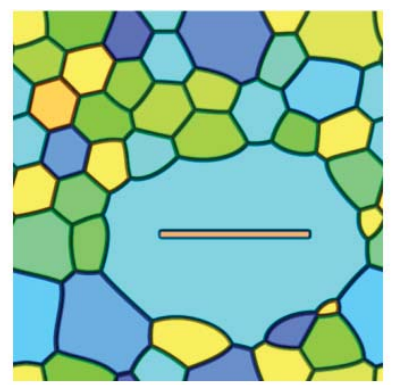

(b) $t^{*}=125$

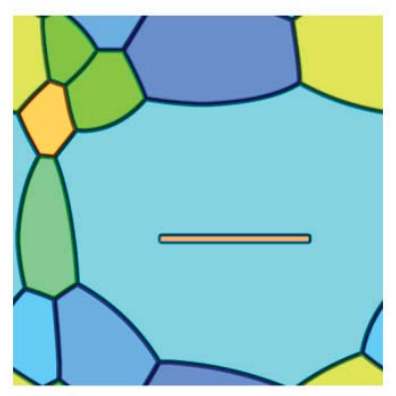

(e) $t^{*}=425$

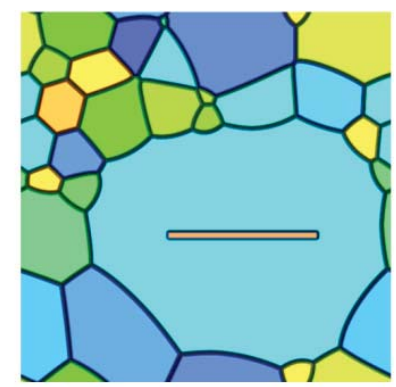

(c) $t^{*}=225$

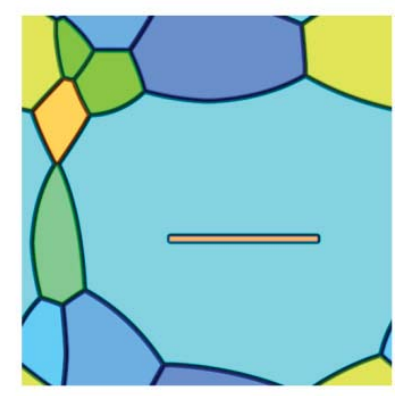

(f) $t^{*}=500$

Figure 4.2 Simulated process of templated grain growth with one template seed per simulation box (volume fraction 0.63\%) [211]. (Refer Appendix E for permission to republish the figure)

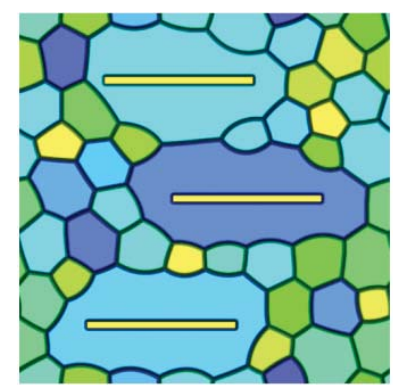

(a) $t^{*}=25$

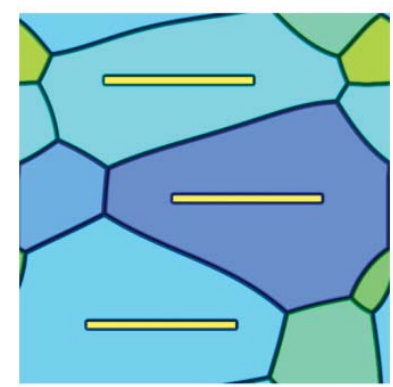

(d) $t^{*}=325$

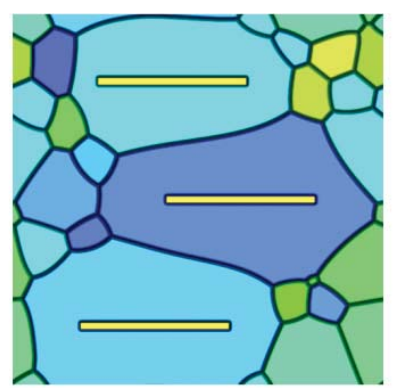

(b) $t^{*}=125$

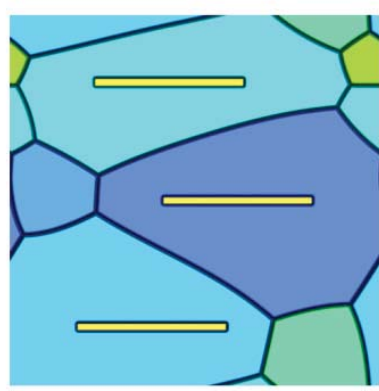

(e) $t^{*}=425$

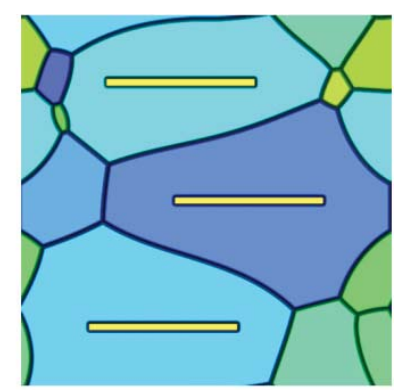

(c) $t^{*}=225$

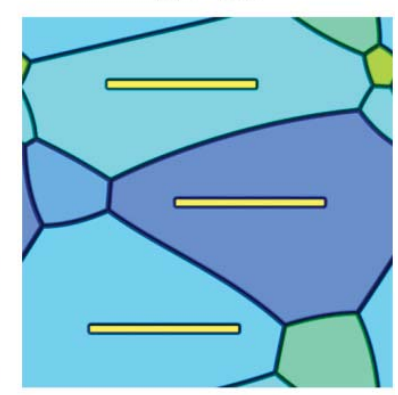

(f) $t^{*}=500$

Figure 4.3 Simulated process of templated grain growth with three template seeds per simulation box (volume fraction $2.37 \%$ ). 


\subsection{X-ray Diffraction Calculation to Characterize Texture Development during Sintering}

In order to characterize the grain texture development in polycrystals during the simulated TGG process, the X-ray diffraction peak intensities are computed for the evolving grain structures as simulated by above described phase field modeling, based on Eqs. (2.8)-(2.11) developed in section 2.3. The described numerical procedure for X-ray diffraction peak intensity calculation provides a convenient means to correlate computational results with experimental measurements. While the simulated polycrystalline grain structures can be directly compared with the experimental observations by imaging techniques such as scanning electron microscopy (SEM) or optical microscopy, microscopy experiments normally require more efforts than X-ray diffraction, thus diffraction is usually the first choice for material characterization. With the peak intensities and Lotgering factor calculated for the evolving grain structures during simulated TGG process, the detailed processes of both grain growth and texture development can be quantitatively investigated by computer simulations and directly compared with X-ray diffraction experiments.

Firstly, to validate the numerical procedure for X-ray diffraction peak intensity calculation as formulated in Eq. (2.10), we consider untextured polycrystals of $\mathrm{ABO}_{3}$ perovskite prototypical of ferroelectrics and compare the calculated peak intensities with powder diffraction database. The structure factor of $\mathrm{ABO}_{3}$ perovskite is [197]:

$$
F_{h k l}=f_{\mathrm{A}}+f_{\mathrm{B}} e^{i \pi(h+k+l)}+f_{\mathrm{O}}\left[e^{i \pi(h+k)}+e^{i \pi(k+l)}+e^{i \pi(h+l)}\right],
$$


where $f_{\mathrm{A}}, f_{\mathrm{B}}$ and $f_{\mathrm{O}}$ are the respective atomic scattering factors of A-site atom, B-site atom and oxygen atom. Fig. 4.4 shows the calculated integrated intensities for the first 6 peaks of untextured polycrystals of strontium titanate $\mathrm{SrTiO}_{3}$ (lattice parameter $a=3.905 \AA$, Cu $K \alpha$ wavelength $\lambda=1.541 \AA$ ) and comparison with Powder Diffraction File, namely, PDF\#84-0444 (calculated) and PDF\#35-0734 (measured), which shows good agreement.

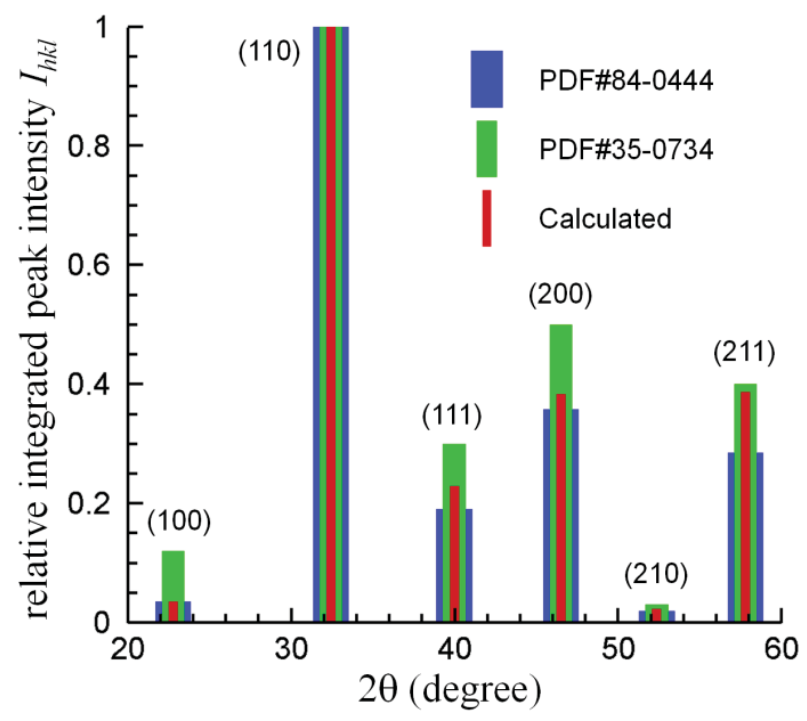

Figure 4.4 Calculated integrated peak intensities (represented by bar height) of untextured $\mathrm{SrTiO}_{3}$ polycrystals and comparison with Powder Diffraction File (PDF) [211]. (Refer Appendix E for permission to republish the figure)

To characterize the texture developments in polycrystals during the grain growth processes shown in Figs. 4.1-4.3, the X-ray diffraction peak intensities are calculated for the evolving grain structures. In relevance to the experiments, we consider $\mathrm{Pb}\left[\left(\mathrm{Mg}_{1 / 3} \mathrm{Nb}_{2 / 3}\right)_{1-x} \mathrm{Ti}_{x}\right] \mathrm{O}_{3}$ (PMN-xPT, $\left.x=32.5 \%\right)$ polycrystals with $\mathrm{BaTiO}_{3}$ template seeds. The effective atomic scattering factor of B-site atom in the perovskite solid solution PMN$x \mathrm{PT}$ is taken as: 


$$
f_{\mathrm{B}}=(1-x)\left(\frac{1}{3} f_{\mathrm{Mg}}+\frac{2}{3} f_{\mathrm{Nb}}\right)+x f_{\mathrm{Ti}} .
$$
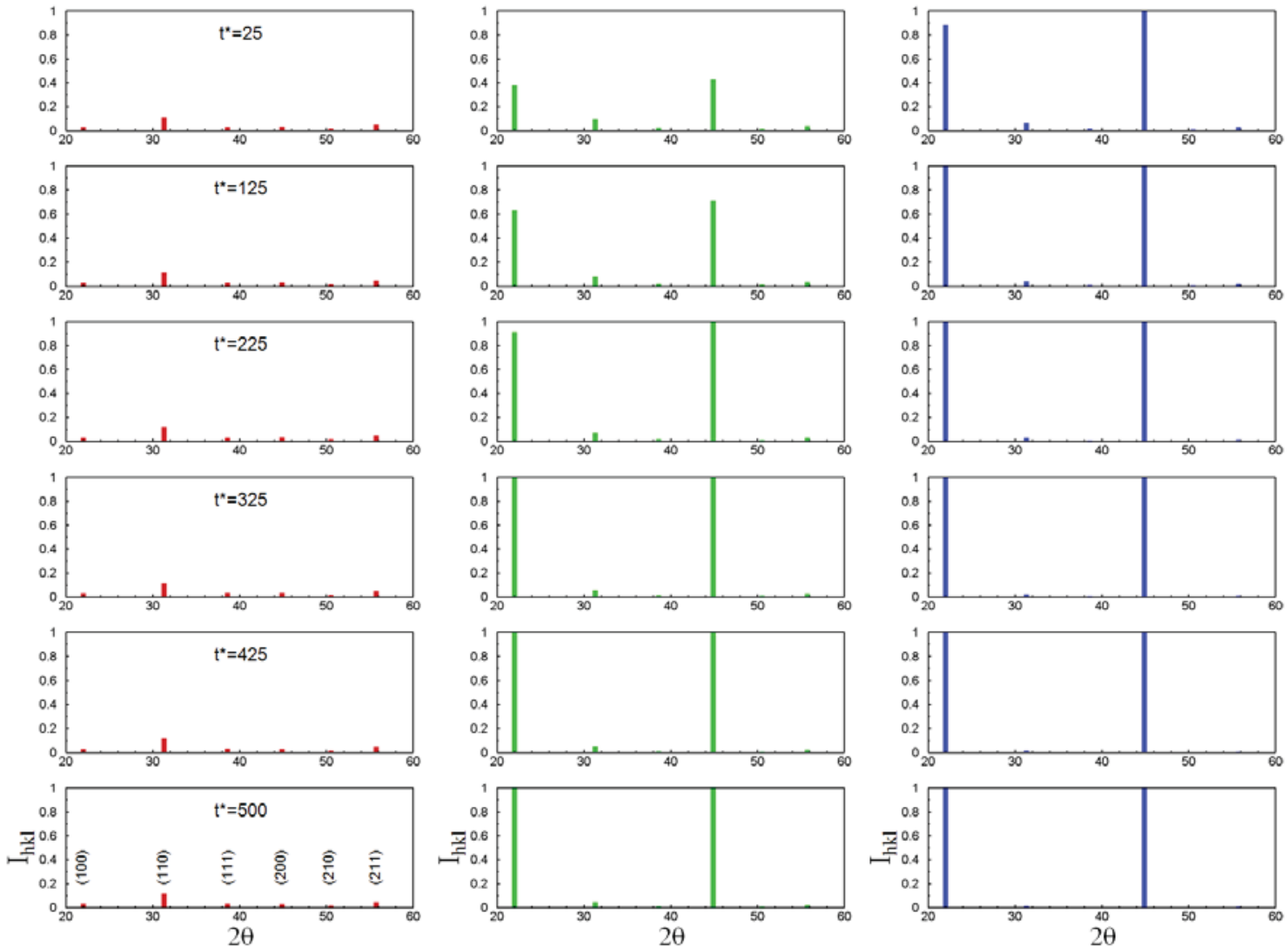

(a) $0 \%$

(b) $0.63 \%$

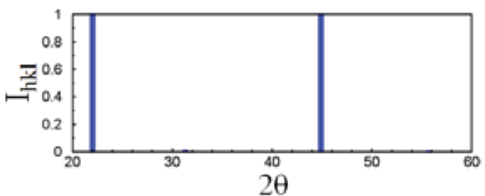

(c) $2.37 \%$

Figure 4.5 Calculated evolutions of X-ray diffraction peak intensities during grain growth processes corresponding to the evolving grain structures in Figs. 4.1, 4.2 and 4.3, respectively. Template volume fraction: (a) $0 \%$, (b) $0.63 \%$, and (c) $2.37 \%$.

Fig. 4.5 shows the evolutions of the calculated X-ray diffraction peak intensities during grain growth processes corresponding to the evolving grain structures shown in Figs. 4.1, 4.2 and 4.3, respectively. Based on the peak intensities, the corresponding values of the Lotgering factor are calculated and plotted in Fig. 4.6, which reveal the gradual development of uniaxial texture in the polycrystals during TGG process. As expected, the 
conventional grain growth without template seed does not produce texture, and the higher the template volume fraction, the faster the texture development, and the higher the degree of final texture.

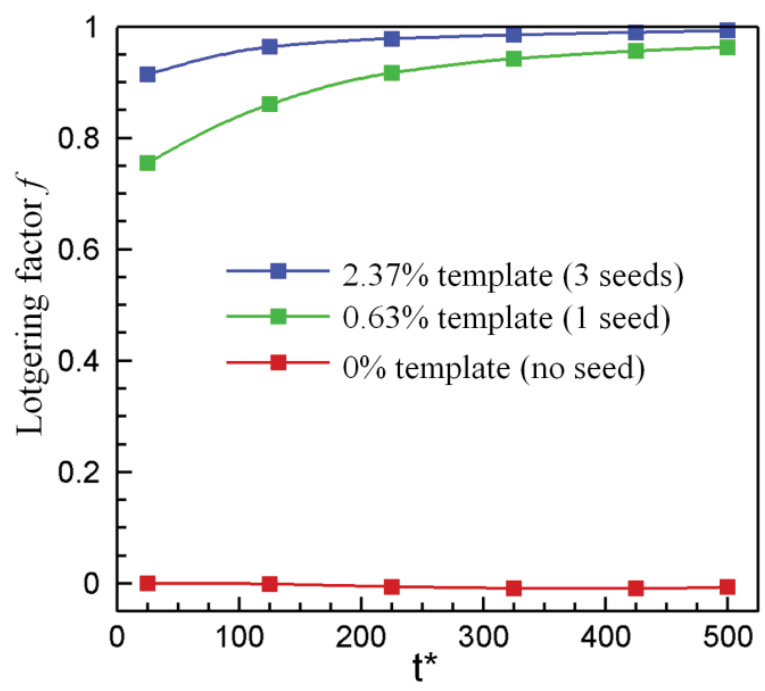

Figure 4.6 Evolution of Lotgering factor during grain growth processes corresponding to the evolving grain structures in Figs. 4.1, 4.2 and 4.3, respectively, as calculated from the X-ray diffraction peak intensities in Fig. 4.5.

To investigate the relation between texture and template volume fraction, a series of simulations are systematically carried out with different template volume fractions and template seed sizes. Fig. 4.7 presents the values of Lotgering factor versus template volume fraction in fully sintered polycrystals (at simulation time $t^{*}=500$ ). It is shown that Lotgering factor rapidly increases with increasing template volume fraction and approaches saturation after $1 \%$ template $(f \rightarrow 1$ implying perfect uniaxial texture). More importantly, it reveals that it is the average distance between template seeds, rather than the template volume fraction, that play a crucial role in texture development. As shown in Fig. 4.7, with the same number of template seeds, the template volume fraction (template 
seed size) does not significantly affect the Lotgering factor; in contrast, at the same template volume fraction, the number of template seeds produces a significant effect on the Lotgering factor. This behavior is understandable in light of the average seed distance: with more template seeds present (thus higher seed number density), the average distance between seed decreases, and the reoriented grains need to grow a shorter distance before impinging onto each other, thus texturing more polycrystal volume in shorter time. Such an effect is more prominent in intermediately sintered polycrystals (at simulation time $t^{*}=225$ ), as shown in Fig. 4.8. Fig. 4.9 shows the simulated evolution of Lotgering factor in polycrystals during TGG processes with the same $2.1 \%$ template volume fraction but different template seed numbers (thus different template seed sizes). It is observed that higher seed number density thus shorter average seed distance leads to faster texture development. This finding has important implication for optimizing TGG processing to achieve better piezoelectric properties in textured ceramics. Since the template phase usually possesses material properties inferior to the matrix phase, a higher volume fraction of templates tends to decrease the properties of the textured ceramics due to composite effect, eroding the advantage of texturing [125]. Therefore, a high degree of texturing achieved at low template volume fraction is of critical importance in practice. Above computational studies show that reducing the template size and shortening seed distance is an effective way to achieve higher texture at lower template volume fraction, enabling further improvement in piezoelectric properties of textured ceramics. 


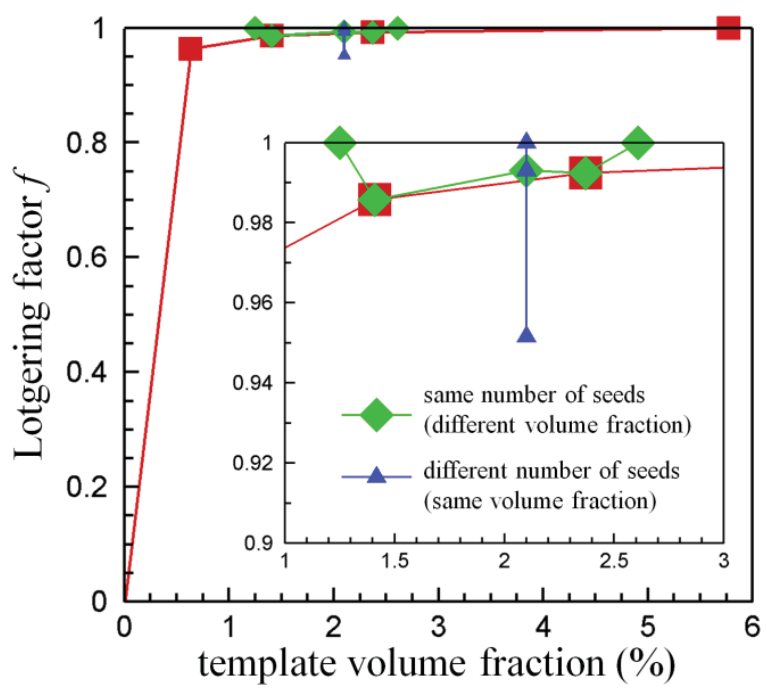

Figure 4.7 Effects of template volume fraction and template seed number on Lotgering factor in fully sintered $\left(t^{*}=500\right)$ polycrystals, based on simulated templated grain growth.

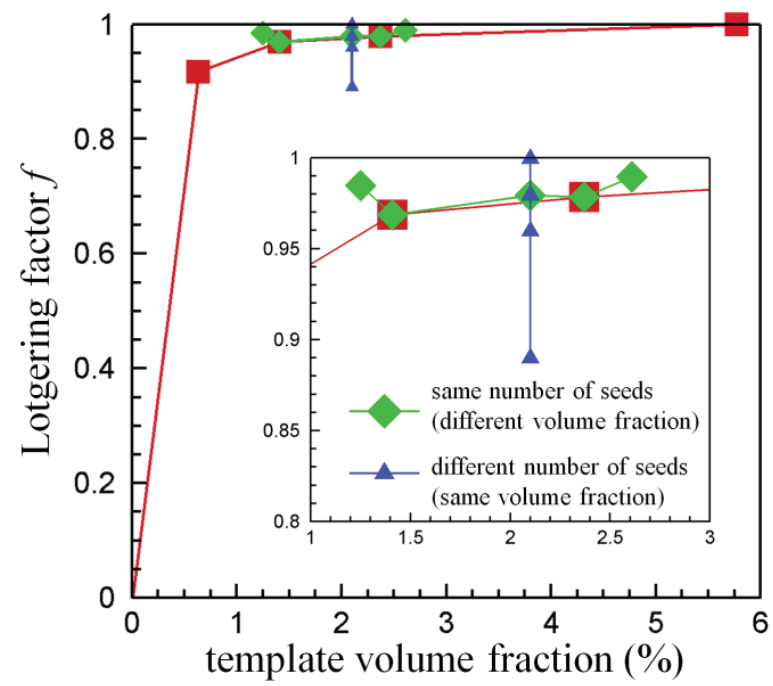

Figure 4.8 Effects of template volume fraction and template seed number on Lotgering factor in intermediately sintered $\left(t^{*}=225\right)$ polycrystals, based on simulated templated grain growth. 


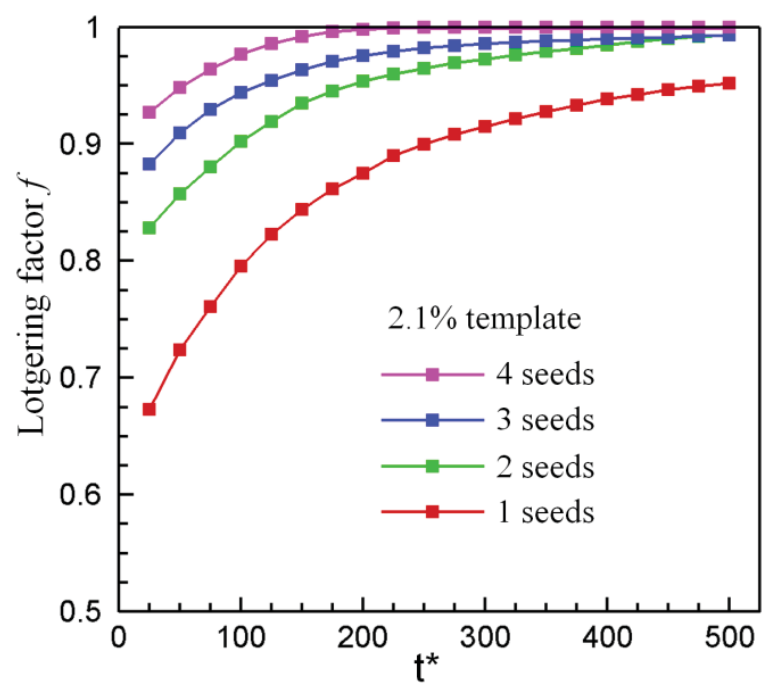

Figure 4.9 Simulated evolution of Lotgering factor during template grain growth processes of same template volume fraction $(2.1 \%)$ but different template seed numbers (thus different template seed sizes).

\subsection{Simulation Validation by Comparison with Experiment}

In order to complement the computational study presented above and further validate our model, experiments are performed by our collaborators (Y. Yan and S. Priya at Virginia Tech.) to characterize the grain structures and textures at different sintering stages. PMN-32.5\%PT ceramics are textured by TGG using 3\% volume fraction of BT templates. Since grain growth is sensitive to sintering temperature, to enhance the experimental sensitivity to sintering time, fast sintering is employed, where the sample is directly put into and taken out of sintering temperature to minimize the effects of heating and cooling. Moreover, a relatively low sintering temperature of $900^{\circ} \mathrm{C}$ is chosen to increase the microstructure difference at different sintering stages. The grain structures are observed on the fractured cross-sections of the ceramic samples at different sintering time by using SEM (FEI Quanta 600 FEG). Fig. 4.10 shows the evolution of grain structures 
during grain growth as function of sintering time. Fig. 4.10(a)-(c) shows the heterogeneous nucleation and epitaxial growth of the neighboring matrix grains on the template surfaces, merging to form reoriented big grains of the same orientation of the templates. This is the stage that is not treated in the mesoscale phase field modeling of grain growth; instead, this experimental observation serves as the basis to create initial states for computer simulations, as presented in Figs. 4.2 and 4.3. Fig. 4.10(d)-(f) shows the subsequent growth of these big grains at the expense of other smaller matrix grains, in agreement with the computer simulations shown in Figs. 4.2 and 4.3. In both simulations and experiments, the reoriented big grains grow rapidly to impinge onto each other, while the randomly oriented smaller matrix grains grow much slower and are retained in the gaps between oriented big grains. It is worth noting that the relative template size with respect to the matrix grain size is greater in the experiments than in the computer simulations; in order to simulate bigger template seeds, a bigger computational box needs to be used, which would drastically increase the requirement for computing resources (memory and time). Nevertheless, despite the smaller templates in the simulations, the computational study captures the essential features of grain structure evolution in TGG process. 

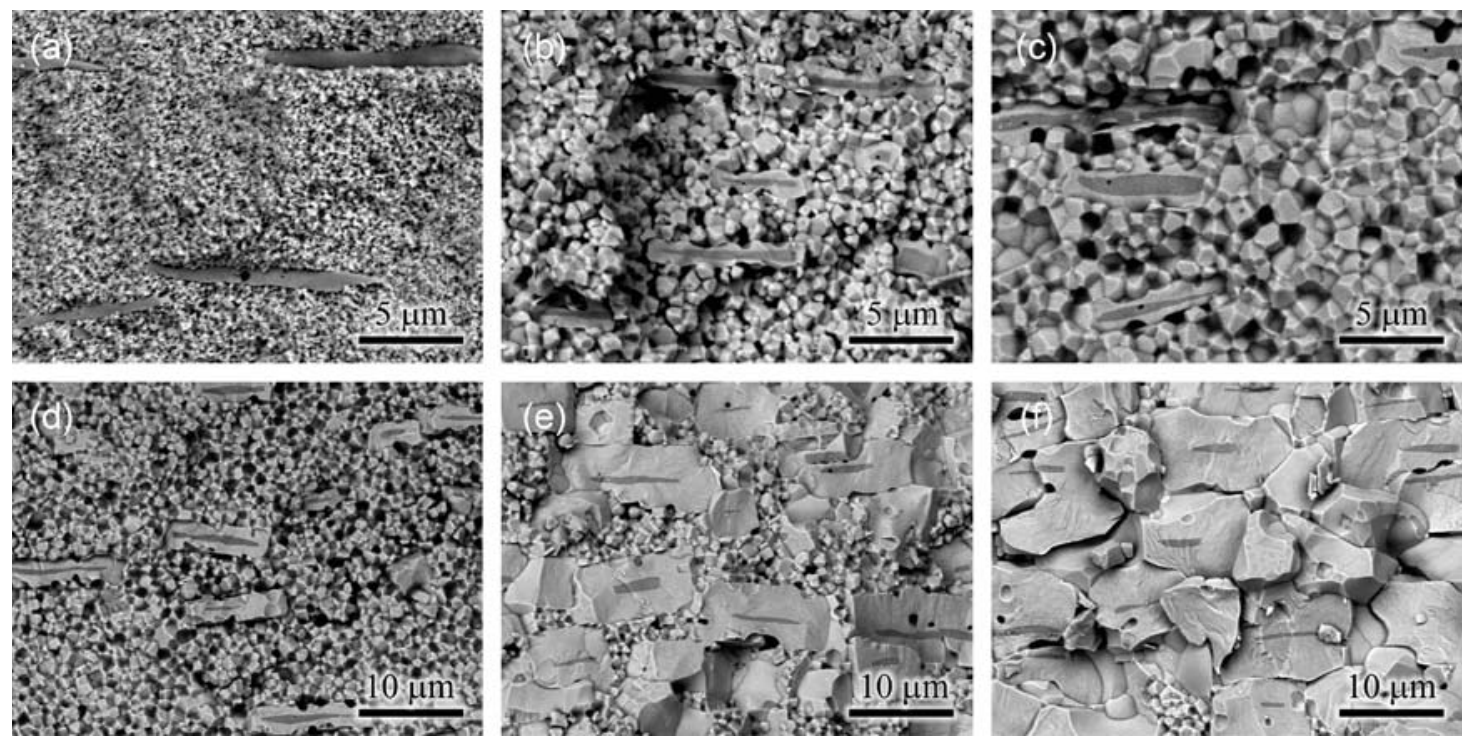

Figure 4.10 SEM images of fractured cross-sections of PMN-32.5\%PT polycrystalline ceramics with $3 \%$ volume fraction of BT templates sintered at $900^{\circ} \mathrm{C}$ for different time $t$ : (a) $1 \mathrm{~min}$, (b) $3 \mathrm{~min}$, (c) $5 \mathrm{~min}$, (d) $10 \mathrm{~min}$, (e) $20 \mathrm{~min}$, and (f) $40 \mathrm{~min}$. (Supplied by Y. Yan and S. Priya at Virginia Tech. Refer Appendix G for the permission to use)

To characterize the texture development in above samples sintered at $900^{\circ} \mathrm{C}$ for different time, X-ray diffraction patterns are measured (XRD, PANalytical X'Pert) by our collaborators on the samples corresponding to the grain structure evolution sequences shown in Fig. 4.10. Fig. 4.11 plots the experimental X-ray diffraction patterns (also from our collaborators) as function of sintering time. To better reveal the pattern features, each curve is normalized with respect to the maximum intensity of its own full intensity profile. Such normalization does not affect the calculation of Lotgering factor. Fig. 4.12 plots the evolution of Lotgering factor with sintering time as determined from the experimental diffraction patterns in Fig. 4.11. 


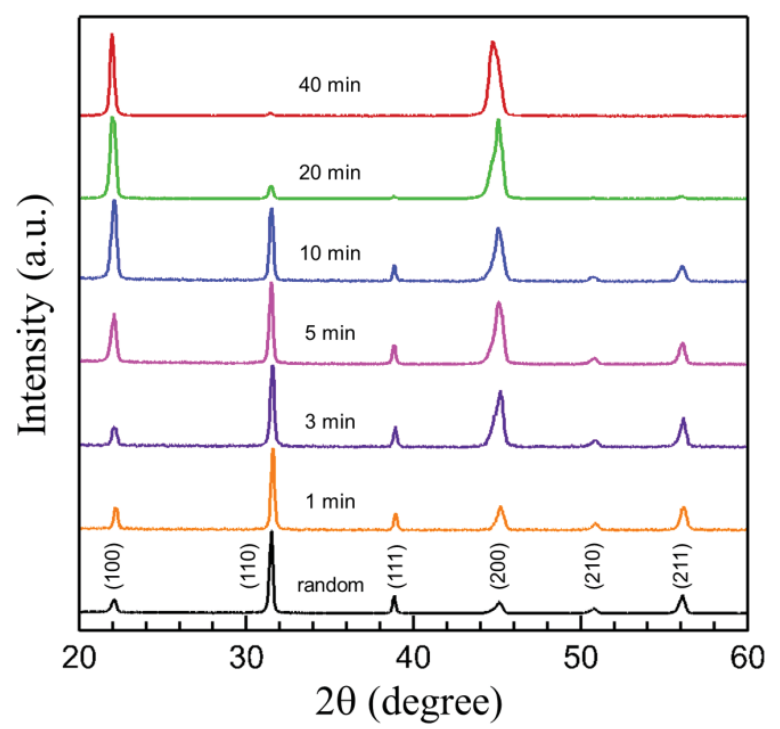

Figure 4.11 Experimental X-ray diffraction patterns of PMN-32.5\%PT polycrystalline ceramics with $3 \%$ volume fraction of BT templates sintered at $900^{\circ} \mathrm{C}$ for different time. (Supplied by Y. Yan and S. Priya at Virginia Tech.)

To quantitatively compare the experimental and computational results, the simulated evolution of Lotgering factor is also plotted in Fig. 4.12. In particular, the red curve in Fig. 4.9 is considered, whose template volume fraction and seed number density are comparable to the experimental samples. To compare with the experimental curve, the reduced simulation time $t^{*}$ is rescaled to fit with the experimental time $t, t=\kappa t^{*}+t_{0}$, where $\kappa$ and $t_{0}$ are fitting parameters. The shifting parameter $t_{0}$ is associated with the fact that, as discussed previously, the simulation characterizes the grain structure evolution during grain growth process and the texture development, while the heterogeneous nucleation of matrix phase on the template surface is a phenomenon of different time and length scales thus is not treated in the mesoscale phase field modeling. As seen from Fig. 4.12, except for an outlying data point at 10 min sintering time, good agreement between computation and experiment is obtained. Such agreement not only validates the phase field modeling, 
but also provides a means to calibrate the time scale of the computer simulation for TGG process. In this work, the computational result obtained at reduced simulation time $t^{*}=500$ corresponds to the experimental sample sintered for about $30 \mathrm{~min}$.

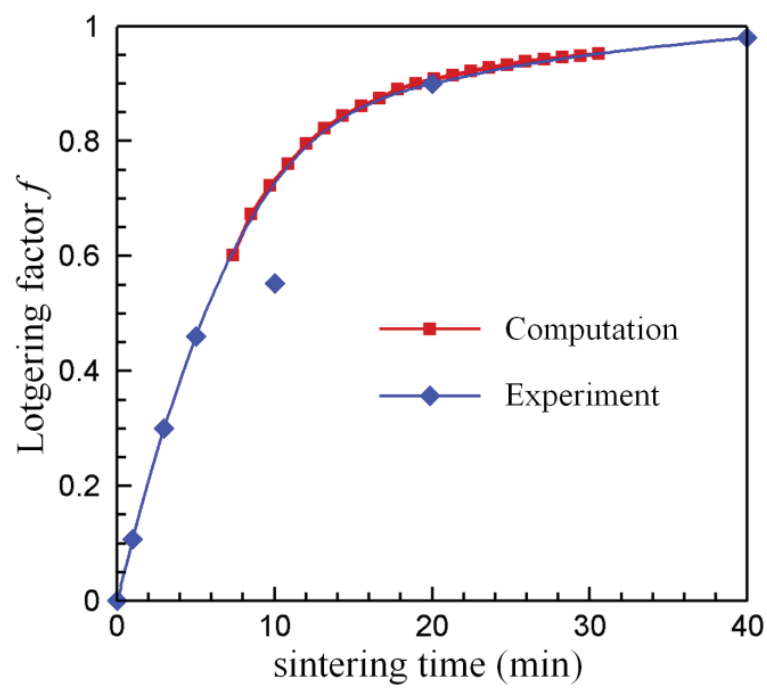

Figure 4.12 Comparison of experimental and computational evolutions of Lotgering factor with sintering time [211]. (Refer Appendix E for permission to republish the figure)

\subsection{Conclusion}

Computer simulations are performed to study templated grain growth process and texture development in ferroelectric polycrystalline ceramics, with good agreements obtained between the results of these complementary computational methods and the experiments performed by our collaborators at Virginia Tech. The effects of template volume fraction and seed sizes on the final grain structure and texture are investigated. It is found that, while the degree of crystallographic texture generally increases with increasing template volume fraction, it is the average template seed distance that plays a direct role, thus reducing the template size and shortening seed distance provides an 
effective means to achieve higher texture at lower template volume fraction, enabling further improvement in piezoelectric properties of textured ceramics. The good agreements between computations and experiments also validate the phase field modeling and computer simulation as an efficient tool to systematically investigate the templated grain growth technique, which offers quantitative details that are difficult and/or costly for experimental methods to obtain. 


\section{Chapter 5. Effects of Crystallographic Texture and Grain Shape Anisotropy on Piezoelectric Properties of Ferroelectric Polycrystalline Ceramics ${ }^{\S}$}

As discussed in Chapter 4, templated grain growth (TGG) is used to generate grain textures for polycrystals in order to exploit their anisotropy properties, which are economic for materials $\mathrm{Pb}\left[\left(\mathrm{Mg}_{1 / 3} \mathrm{Nb}_{2 / 3}\right)_{1-x} \mathrm{Ti}_{x}\right] \mathrm{O}_{3}$ and $\mathrm{Pb}\left[\left(\mathrm{Zn}_{1 / 3} \mathrm{Nb}_{2 / 3}\right)_{1-x} \mathrm{Ti}_{x}\right] \mathrm{O}_{3}$ whose single crystals are costly, and even more critical for $\mathrm{Pb}\left(\mathrm{Zr}_{1-x} \mathrm{Ti}_{x}\right) \mathrm{O}_{3}$ (PZT) whose single crystal is not available. Texturing is particularly important for improving piezoelectric properties of ferroelectric polycrystals and developing lead-free piezoelectrics. In this Chapter, phase field modeling and computer simulation is employed to study the effects of crystallographic textures and grain shapes, which usually accompany texturing, on the piezoelectric properties of ferroelectric polycrystals. Domain evolutions and polarizationelectric field $(P-E)$ and strain-electric field $(\varepsilon-E)$ hysteresis loops are simulated for polycrystals of different grain textures and shape aspect ratios. The coercivity, remanence, dielectric permittivity and piezoelectric coefficient are studied as functions of grain texture and shape aspect ratio. It is found that crystallographic texture significantly affects polycrystal properties, while grain shape does not play a significant role. The critical degree of texturing is determined for achieving single crystal-like properties. The obtained

\footnotetext{
$\S$ The material contained in Chapter 5 will be submitted in Effects of Crystallographic Texture and Grain Shape Anisotropy on Piezoelectric Properties of Ferroelectric Polycrystalline Ceramics, by Jie E. Zhou, Yu U. Wang.
} 
quantitative information helps optimize the design and processing of textured polycrystalline ceramics.

\section{Model Preparation:}

The state of ferroelectric polycrystal is described by a polarization field $\mathbf{P}(\mathbf{r})$, whose total system free energy under externally applied electric field $\mathbf{E}^{\text {ex }}$ is adopted from Eq. (2.2). The spatial-temporal evolution of polarization $\mathbf{P}(\mathbf{r}, t)$ is characterized by the timedependent Ginzburg-Landau equation from Eq. (2.4). In this Chapter, we consider PZT of composition $x=0.6$ at room temperature, whose material parameters have been experimentally determined [195].

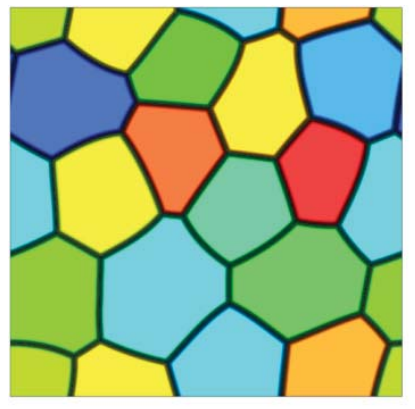

(a) Equiaxed (1:1)

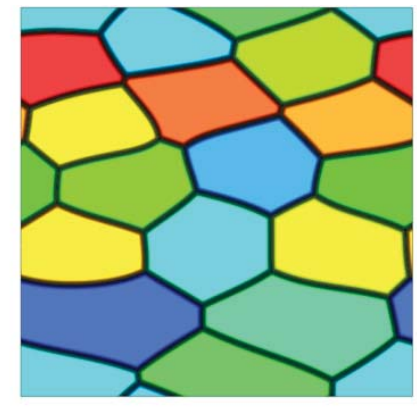

(b) Elongated (2:1)

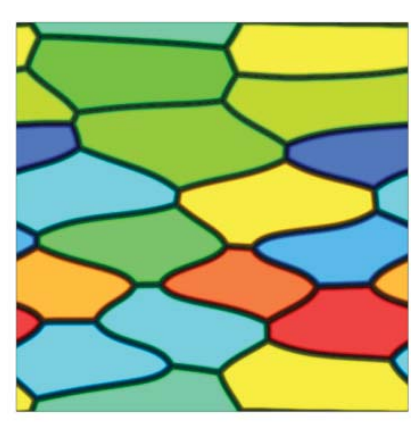

(c) Elongated (4:1)

Figure 5.1 Polycrystalline grain structures of different grain shape anisotropies, namely, (a) equiaxed grains of average shape aspect ratio 1:1, and elongated grains of average shape aspect ratio (b) $2: 1$ and (c) 4:1.

To investigate the effects of grain shape and texture, 16 grains of different shape anisotropies and orientation distributions are simulated under periodic boundary conditions. Fig. 5.1 shows the polycrystalline grain structures of different grain shape aspect ratios (i.e., 1:1, 2:1, 4:1) used in the computer simulations. For each grain structure, 
different degrees of grain texture are considered. In this Chapter, uniaxial texture is considered, which is important for piezoelectric ceramics and can be achieved by templated grain growth technique. To quantify the degree of texturing, the grain orientations are randomly generated with their [010] axes distributed within the solid angle of a cone with half apex angle $\theta$, which measures the degree of uniaxial texture used in the computer simulations (while the [100] and [001] axes are randomly rotated around the [010] axis). Fig. 5.2 plots the stereographic projections of the (010) plane orientation distributions for representative examples of untextured $\left(\theta=90^{\circ}\right)$, intermediately textured $\left(\theta=20^{\circ}\right)$ and highly textured $\left(\theta=5^{\circ}\right)$ grains.

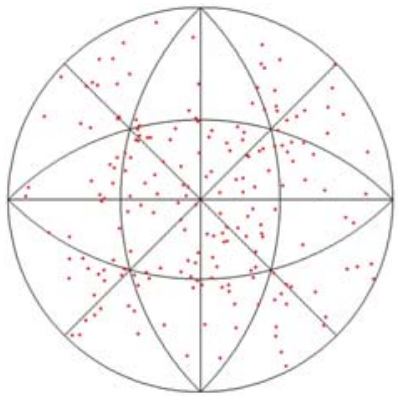

(a) Untextured $\left(90^{\circ}\right)$

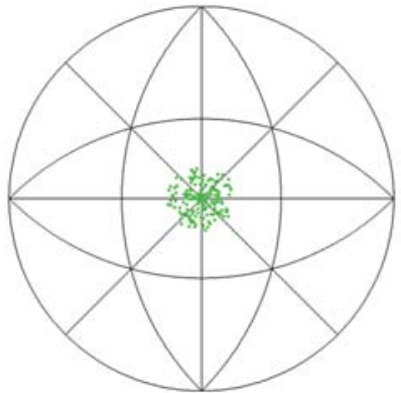

(b) Textured $\left(20^{\circ}\right)$

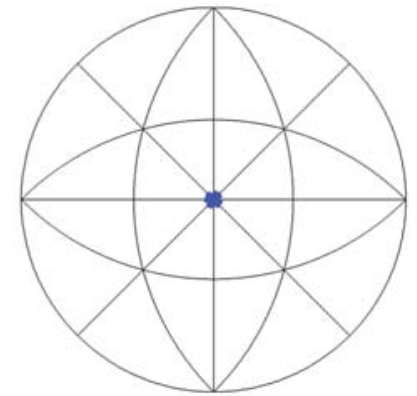

(c) Textured $\left(5^{\circ}\right)$

Figure 5.2 Stereographic projections of (010) plane orientation distributions for (a) untextured $\left(\theta=90^{\circ}\right)$, (b) intermediately textured $\left(\theta=20^{\circ}\right)$, and (c) highly textured $\left(\theta=5^{\circ}\right)$ grains.

\subsection{Grain Shape Effect on Ferroelectric Properties}

The effect of grain shape anisotropy is first investigated. Inevitably, the grain shape effect is usually coupled with grain orientation effect (texture). In order to derive the effect of grain shape out of the coupled effects, special geometry setting up for calculation is 
adopted as illustrated in Fig. 5.3. The upper row (a-c) of Fig. 5.3 is the same grain structures shown in Fig. 5.1. The lower row $\left(\mathrm{a}^{\prime}-\mathrm{c}^{\prime}\right)$ represents the "mirror" structures of upper row (ac) respectively. We call structure ' $\mathrm{B}$ ' the mirror of structure ' $\mathrm{A}$ ' if ' $\mathrm{B}$ ' is generated from 'A' by a mirror operation about diagonal of ' $A$ ' itself. The crystallography axis assigned previously for each grain stay the same for all the grain structures shown in Fig. 5.3 such that when measuring material properties along certain direction, for example [010], the only variable among all the grain structures in Fig. 5.3 is the grain shape along that direction without texture effect involved. Also, as will be shown below, to use identical texture for all grain structures helps us to further identify the shape effect with different extent of grain shape anisotropy.

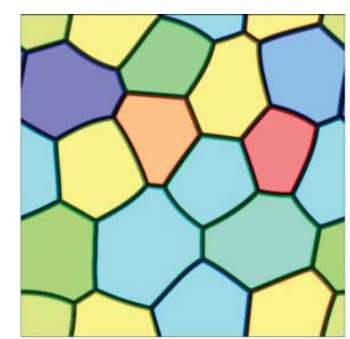

(a) Ratio (1:1)

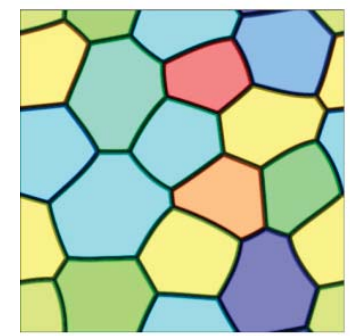

(a') Mirror of (a)

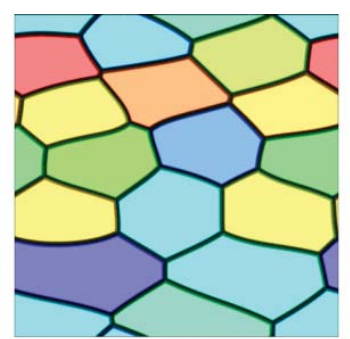

(b) Ratio (2:1)

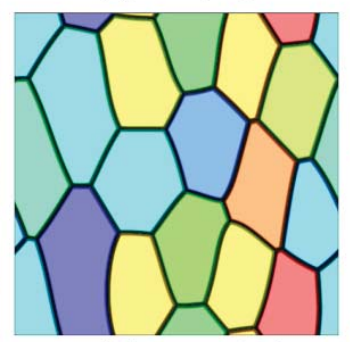

(b') Mirror of (b)

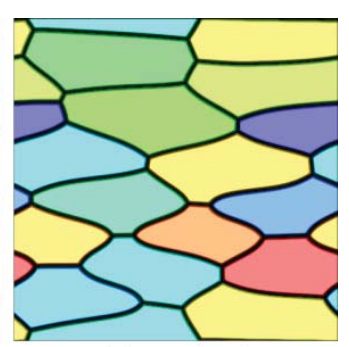

(c) Ratio (4:1)

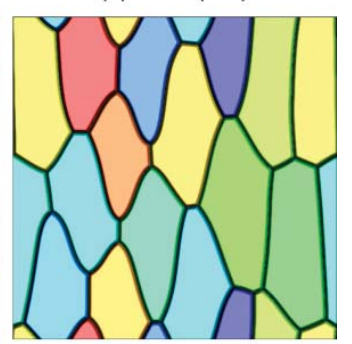

(c') Mirror of (c)

Figure 5.3 (a) Equiaxed (1:1) grain structure shown in Fig. 5.1. (a') Corresponding to mirror operation about the diagonal of (a) itself. (b) Elongated (2:1) grain structure shown in Fig. 5.1. (b') Corresponding to mirror operation about the diagonal of (b) itself. (c) Elongated (4:1) grain structure shown in Fig. 5.1. (c') Corresponding to mirror operation about the diagonal of (c) itself. 
Simulated $P-E$ and $\varepsilon-E$ hysteresis loops are presented in Fig. 5.4 based on grain structures of Fig. 5.3. Green curves represent normal grain structure while red ones represent their mirror. Fig. 5.4(a) with the grain structure obtained through voronoi tessellation algorithm shows no grain shape anisotropy effect from the fact that $P$-E hysteresis loops for equiaxed grain structure and its mirror are nearly coincident with each other. Material properties, such as coercivity field and remnance polarization measured along [100] direction, are higher with mirror grain structure compared to normal grain structure from Figs. 5.4(b) and (c). Similarly, Figs. 5.4(e) and (f) show that strain response with mirror grain structure along [100] direction is also higher compared with its normal grain structure counterpart. It is worth to mention that the "mismatch" between the two butterfly strain curves on Fig. 5.4(d) for equiaxed grain structure is due to the incomplete or imperfect grain shape statistic for equiaxed grain structure system. Such "mismatch" is acceptable because it does not prevent us from qualitatively explaining grain shape effect. In the real application, the absolute value of strain is of less importance compared with the relative change of strain (response) when applying external field. Figs. 5.4(g), (h) and (i) are for the purpose to better show the strain response with different grain shapes by a curve shifting operation on Figs. 5.4(d), (e) and (f) respectively. The results show that the normal grain structure possesses higher responses compared with its mirror, while the mirror grain structure has the higher absolute value of the measured properties. To facilitate our analysis, grain shape effect could be alternatively redefined to be a proposition of the effect with different density of grain boundaries along the measured direction such as [100]. The conclusion could be drawn that the higher the density of grain boundaries along [100], the 
larger the absolute values of coercivity field, remnance polarization and strain would be as we can see from Fig. 5.4, however, the ferroelectric response properties such as dielectric and piezoelectric constants show the opposite trend. As already known, the dielectric and piezoelectric properties in ferroelectric materials highly depend on extrinsic factor (domain structure and movement). Density of grain boundaries could affect the morphology of domain distribution, thus materials property as sequence. In order to better demonstrate the domain structure with varying grain boundary densities, ratio (4:1) grain structure and its mirror are selected as representative to show their domain pattern difference during loading. Fig. 5.5 shows selected snapshots of typical domain patterns to explain the grain shape effect related with extrinsic domain structure. Mirror grain structure always has fewer domain variants compared with normal grain structure when poling along [100] direction, where mirror grain structure possesses higher grain boundary density. Along [100] direction, normal grain structure is elongated, thus, domain patterns could be fully relaxed with more optional domain variants to further eliminate electric and elastic energy by forming twin configuration. However, for mirror grain structure, such freedom for more variants is limited due to smaller grain dimension constrained by grain boundaries along [100] direction. Sandwiched by two relatively close grain boundaries, single domain variant inclines to form due to the potential energy cost if more domain interfaces emerge, leading to a smaller quantity of domain variants in mirror grain structure. More optional domain variants lead to more kinetic pathways for polarization switching [202] resulting in the higher ferroelectric responses, but lower coercivity and remnance, as well as the component of strain along [100]. 


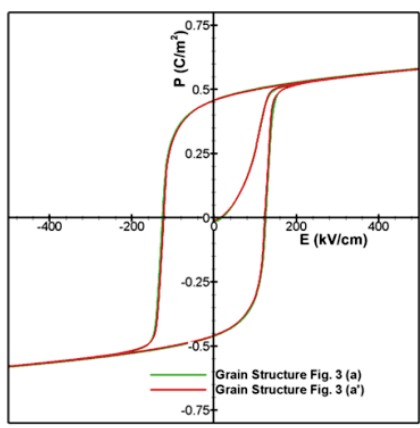

(a)

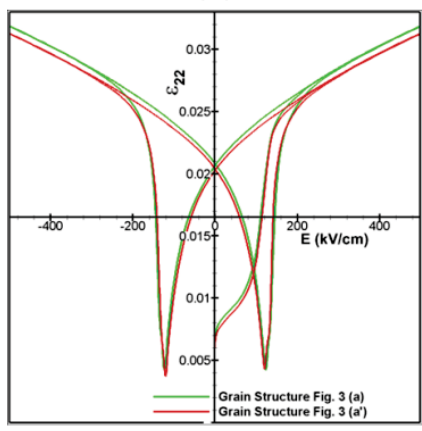

(d)

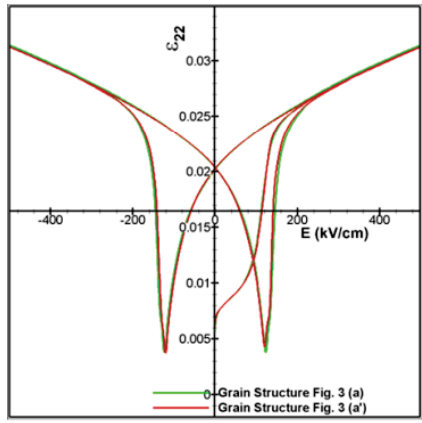

(g)

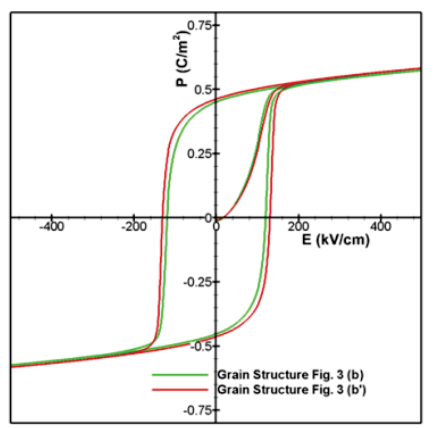

(b)

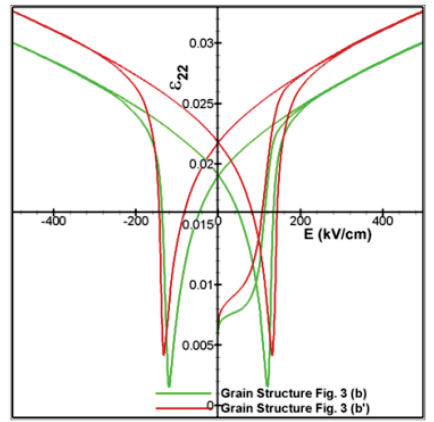

(e)

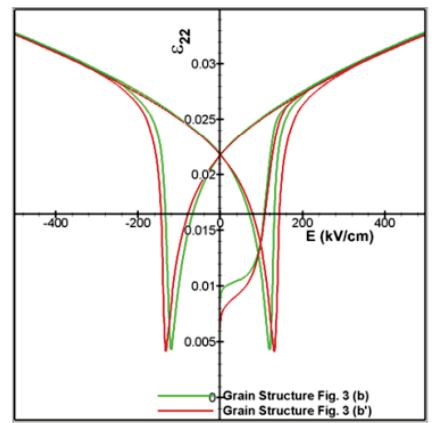

(h)

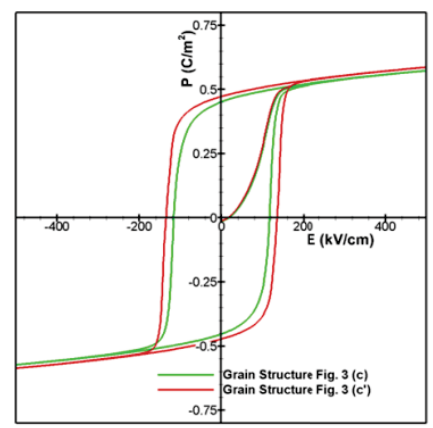

(c)

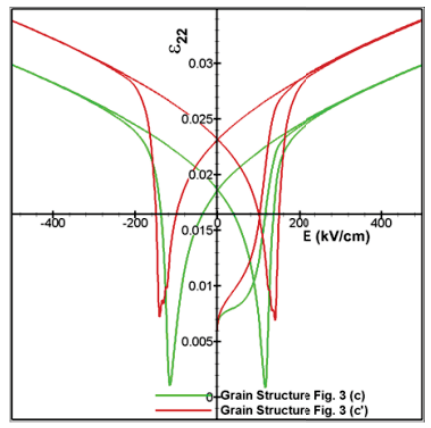

(f)

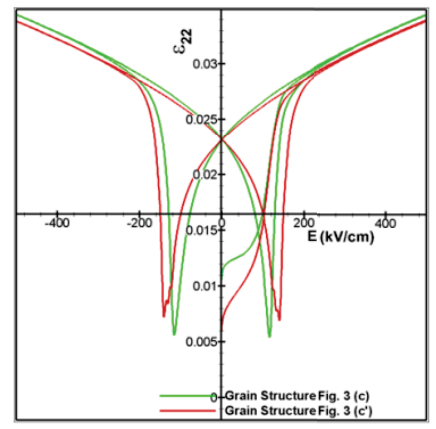

(i)

Figure 5.4 Simulated $P-E$ hysteresis loop measured along [100] based on the (a) Equiaxed grain structure and its mirror. (b) Elongated (2:1) grain structure and its mirror. (c) Elongated (4:1) grain structure and its mirror. Simulated $\varepsilon-E$ hysteresis loop measured along [100] based on the grain structure (d) Equiaxed grain structure and its mirror. (e) Elongated (2:1) grain structure and its mirror. (f) Elongated (4:1) grain structure and its mirror. (g), (h) and (i) are obtained from (d), (e) and (f) respectively by shifting the two $\varepsilon^{-}$ $E$ hysteresis loops on each graph to a common reference strain position. 


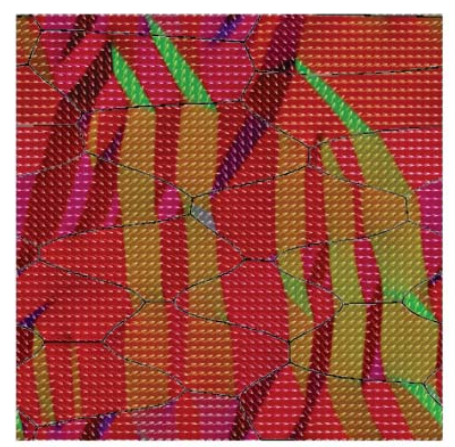

(a) After poling $(\mathrm{E}=0 \mathrm{kV} / \mathrm{cm})$

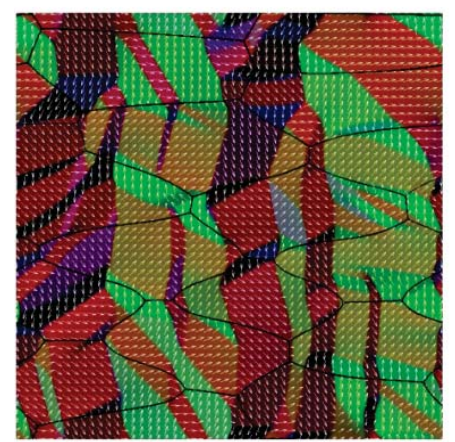

(c) Around switching point

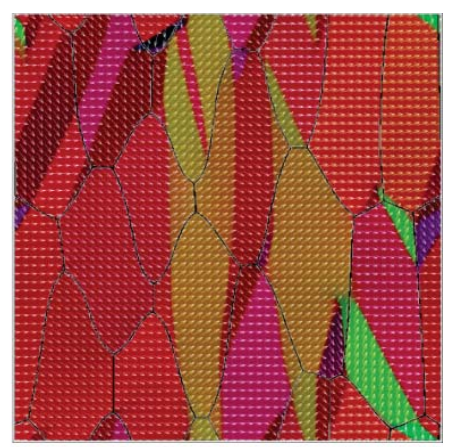

(b) After poling $(\mathrm{E}=0 \mathrm{kV} / \mathrm{cm})$

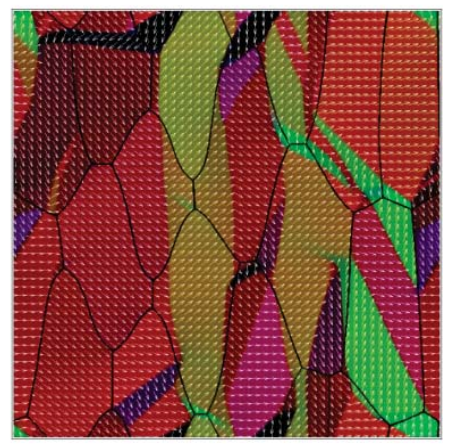

(d) Around switching point

Figure 5.5 Selected typical domain patterns for grain ratio (4:1) (a) After poling $(\mathrm{E}=0 \mathrm{kV} / \mathrm{cm})$ for normal grain structure. (b) After poling $(\mathrm{E}=0 \mathrm{kV} / \mathrm{cm})$ for mirror grain structure. (c) Around switching point for normal grain structure. (d) Around switching point for mirror grain structure. White arrows denote in-plane components of polarization.

Fig. 5.5(a) compared with Fig. 5.5(b) shows the relatively larger amount of domain variants along the direction with lower grain boundary density at the $E$ - $P$ Loop stage after poling $(\mathrm{E}=0 \mathrm{kV} / \mathrm{cm})$. Fig. 5.5(c) compared with Fig. 5.5(d) further demonstrates the relatively larger amount of domain variants along the direction with lower grain boundary density near polarization switching point. 


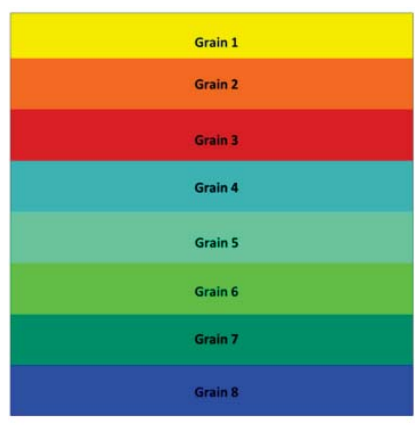

(a)

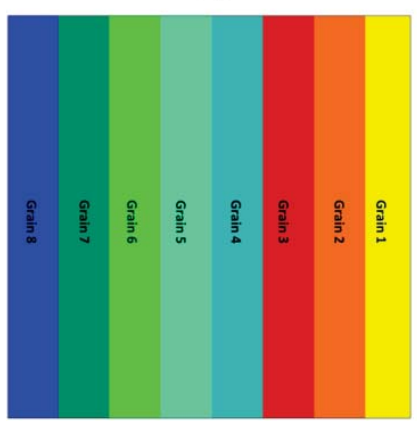

(c)

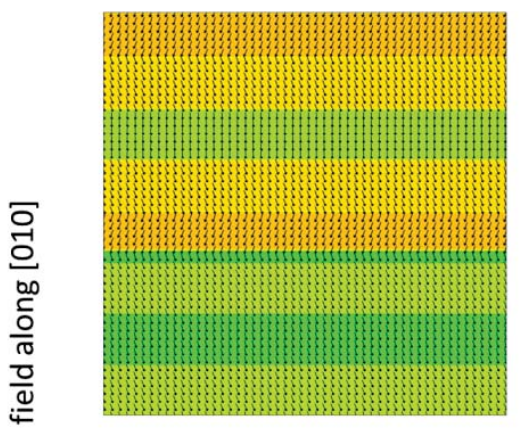

(b)

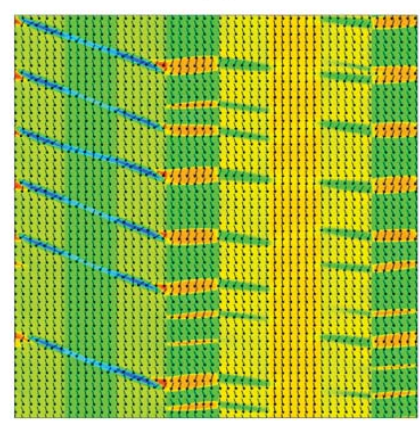

(d)

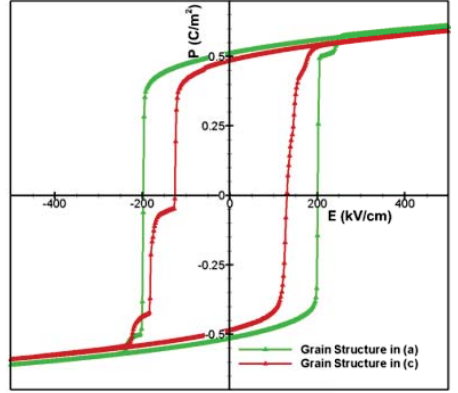

(e)

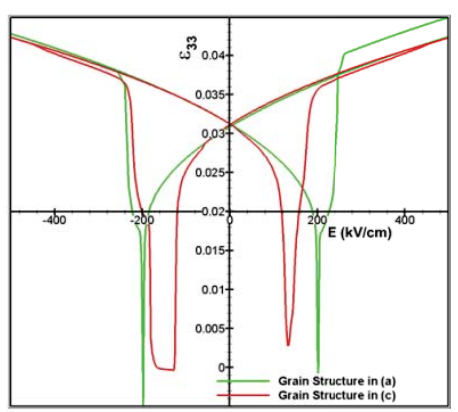

(f)

Figure 5.6 Arrangement of hypothetical grains with infinite shape aspect ratio. Total 8 grains are present and their structural arrangements are designated as (a) horizontal alignment (c) vertical alignment. Corresponding to (a) and (c) respectively, (b) and (d) are domain structures close to switching point of $E-P$ loop measured along [010]. Different grains are labeled with different colors. Domain structure is visualized with $\mathrm{P}_{3}$ as out of plane component, while black arrows represent in-plane polarizations. Rightmost graphs (e) and (f) show $E-P$ hysteresis loops and shifted $\varepsilon-E$ hysteresis loops respectively.

Additionally, as a special case shown in Fig. 5.6 to further confirm the domain configuration features affected by grain shape anisotropy, 8 grains with infinite aspect ratio are chosen to do the calculation. Fig. 5.6(a) and (c) are two ways for grain alignments. Loading direction for both grain structures is along [010]. Grain structure of Fig. 5.6(a) possesses higher grain boundary density along loading direction, while lower in Fig. 5.6(c). Based on previous conclusion that the grain setting up with low grain boundary density 
would contribute to more domain variants than the grain arrangement with high grain boundary density, domain structure in Fig. 5.6(b) thus presents less domain variants along [010] than in Fig. 5.6(d) as expected. Also, for such extreme case, $E-P$ Loops and $\varepsilon-E$ Loops are constructed respectively in Fig. 5.6(e) and (f) to quantitatively confirm the grain boundary density effect on the ferroelectric properties.

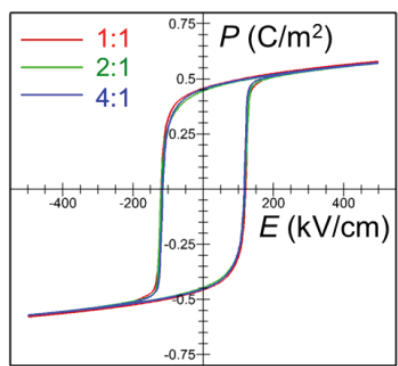

(a) $P$-E along [100]

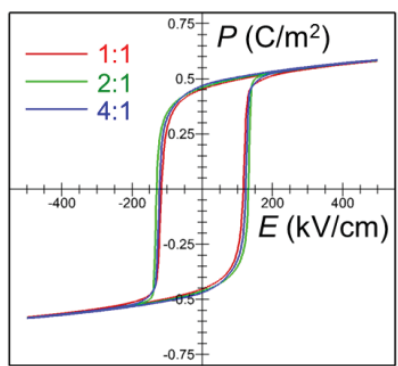

(b) $P$-E along [010]

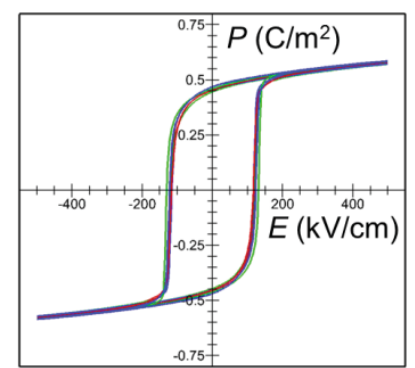

(c) P-E along [100] \& [010]

Figure 5.7 Simulated $P-E$ hysteresis loop for untextured polycrystals of different grain shape aspect ratios $(1: 1,2: 1,4: 1)$ as shown in Fig. 5.1, measured along (a) [100] (horizontal) and (b) [010] (vertical) directions, and plotted all together in (c) for comparison.

Grain shape does contribute to materials properties through affecting the evolution of domain structure, but such effect is minor compared with the texture effect we will discuss in the following section. Untextured $\left(\theta=90^{\circ}\right)$ polycrystals of different grain shape aspect ratios as shown in Fig. 5.1 are simulated to justify the minor shape effect for $P-E$ loop. Figs. 5.7(a) and (b) plot the simulated $P-E$ loop as measured along horizontal [100] and vertical [010] directions, respectively. For comparison, all curves shown in Fig. 5.7(a) and (b) are plotted together in Fig. 5.7(c). There is no prominence difference among the curves belonging to different aspect ratio. The strain curves for different aspect ratio are 
not showing here for simplicity. The strain disparity caused by shape anisotropy is several times lower than that caused by texture which will be discussed in the following section.

\subsection{Grain Texture Effect on Ferroelectric Properties}

In this section, grain texture effect will be discussed. Grain shape aspect ratio (1:1) is selected as our grain structure since texture effect should be mono-factor under investigation. Uniaxial texture type and apex angle $\theta$ (hereafter, we also call $\theta$ the distribution angle) are illustrated in Fig. 5.2. To qualitatively study the effect of uniaxial texture, different values of distribution angle from $0^{0}$ (highly textured) to $90^{\circ}$ (random orientated) are selected. Fig. 5.8 shows $P$ - $E$ hysteresis loops and $\varepsilon$ - $E$ hysteresis loops measure along [010] direction with different values of distribution angle. Both $P-E$ and $\varepsilon^{-}$ $E$ hysteresis loops show the strong relations with texture change, presenting the trend of higher texture (lower distribution angle) accompanied with lower remnance, coercivity and strain. An interesting phenomenon could be seen from Figs. 5.8(a) and (b) where loops have an abrupt change when distribution angle changing from $15^{0}$ to $20^{\circ}$. Useful ferroelectric information could be extracted from Figs. 5.8(a) and (b) such as remanence, coercivity, dielectric constant and piezoelectric coefficient as shown in Figs. 5.9(a)-(d) respectively. Remanence and coercivity present the same trend as decreasing with the increasing of distribution angle. Increment of distribution angle will facilitate generation

of more domain variants, thus, reduce the polarization component along measured direction and supply more feasible kinetic pathways for switching behavior, leading to the decrement for both remanence and coercivity. Similarly, for dielectric constant, as a role to measure 
the polarization response to external electric field, it increases with distribution angle. It is worth to note that response curves shown in Figs. 5.9(a)-(c) for remanence, coercivity and dielectric constant respectively present saturation behavior after distribution angle $60^{\circ}$ which indicates that distribution angle greater than $60^{\circ}$ dose not significantly change quantities of ferroelectric property, leading to the approximate results as total random orientation case (distribution angle $90^{\circ}$ ). Fig. 5.9(d) shows the variation of piezoelectric coefficient with distribution angle. Piezoelectric coefficient decreases with the increase of distribution angle, where it obtains its minimum value at distribution angle $90^{\circ}$. Such trend could be explained by the behavior of piezoelectric coefficient coupled with texture development in TGG. In TGG process, $\mathrm{d}_{33}$ increases with the development of highly orientated grains of preferred uniaxial alignments. When more grains are reoriented to preferred uniaxial direction (development of texture), $\mathrm{d}_{33}$ is enhanced along such direction. In this section, high distribution angle represents high orientation randomness, thus, lower chance for grains in the matrix to possess the close alignment. In other words, high distribution angle is analogous to the non-fully developed early stage in TGG with lower amount of highly orientated grains along specific direction, while $0^{0}$ distribution angle designates fully developed TGG with all grains obtaining uniaxial preferred orientations, resulting in the monotonic $\mathrm{d}_{33}$ decrement behavior in Fig. 5.9(d). In addition, to quantitatively investigate the piezoelectric behavior, the intrinsic relation among remnance $\left(\mathrm{P}_{\text {rem }}\right)$, dielectric constant $\left(\varepsilon_{33}\right)$ and piezoelectric constant $\left(\mathrm{d}_{33}\right)$ is expressed as:

$d_{33}=2 \bar{Q}_{11} P_{r e m} \varepsilon_{33}$, 


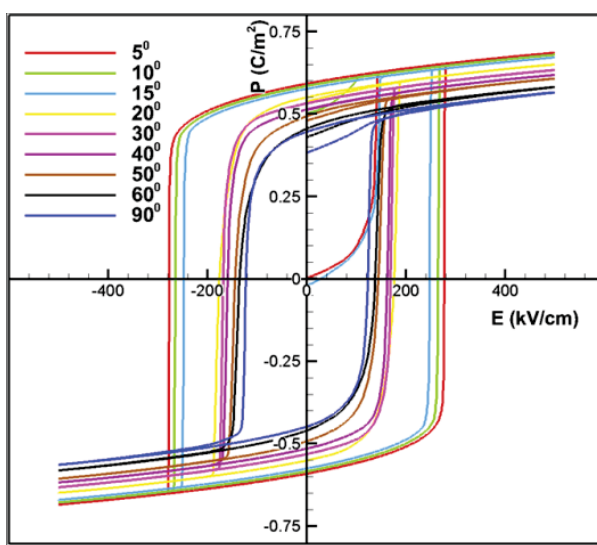

(a)

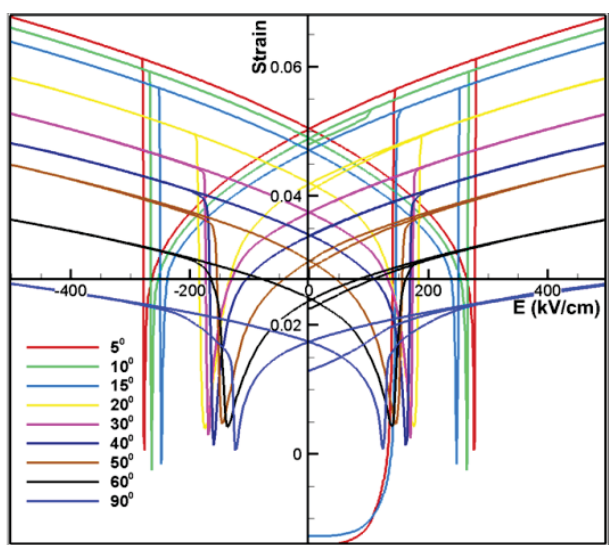

(b)

Figure 5.8 (a) Simulated $P-E$ hysteresis loops for different values of texture for polycrystals of grain shape aspect ratios (1:1). (b) Simulated $\varepsilon-E$ hysteresis loops for different values of texture for polycrystals of grain shape aspect ratios (1:1).

where $\bar{Q}_{11}$ is effective electrostriction for textured polycrystals. For the polycrystal with distribution angle $0^{0}, \bar{Q}_{11}$ has the form $\bar{Q}_{11}=Q_{11}$ representing highly textured case. For the polycrystal with distribution angle $90^{\circ}, \bar{Q}_{11}$ has the form $\bar{Q}_{11}=<Q_{11}>=$ $\left(3 Q_{11}+2 Q_{12}+Q_{44}\right) / 5$ representing highly random case. These two extreme cases for different choice of $\bar{Q}_{11}$ will determine upper and lower bound respectively for $\mathrm{d}_{33}$. For our calculation, $Q_{11}=0.081161 \mathrm{~m}^{4} / \mathrm{C}^{2}, Q_{12}=-0.029498 \mathrm{~m}^{4} / \mathrm{C}^{2}$ and $Q_{44}=0.067103 \mathrm{~m}^{4} / \mathrm{C}^{2}$ are adopted. $\left\langle Q_{11}>\right.$ is then calculated to be $0.050318 \mathrm{~m}^{4} / \mathrm{C}^{2}$ for lower bound. Based on the intrinsic relation shown in Eq. (5.1), the $d_{33}$ values from phase field simulation should be sandwiched by the upper bound curve and lower bound curve shown in Fig. 5.10. However, the $d_{33}$ values from simulation (red curve in Fig. 5.10) are higher than upper bound (blue curve in Fig. 5.10) when distribution angle is smaller than $60^{\circ}$. The reason for such deviation is that $\mathrm{d}_{33}$ values from phase field simulation automatically take domain 
wall motion as consideration, while Eq. (5.1) only considers intrinsic property without involving kinetic effect. The result further confirms the conclusion that domain wall motion significantly contributes to ferroelectric responses.

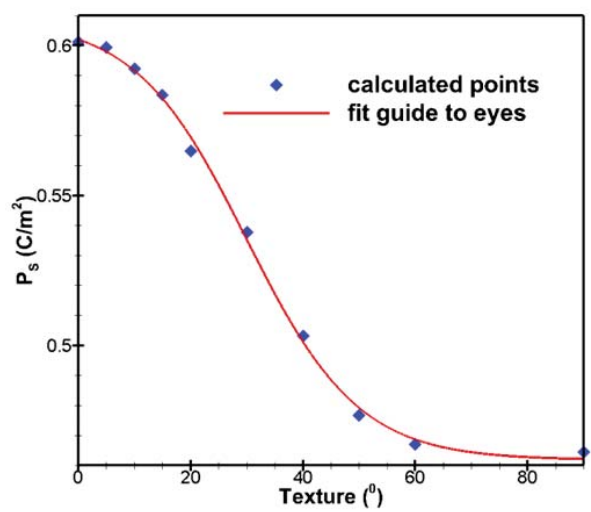

(a)

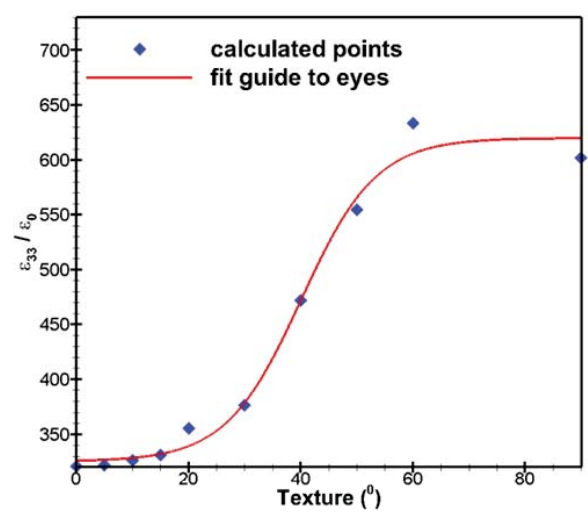

(c)

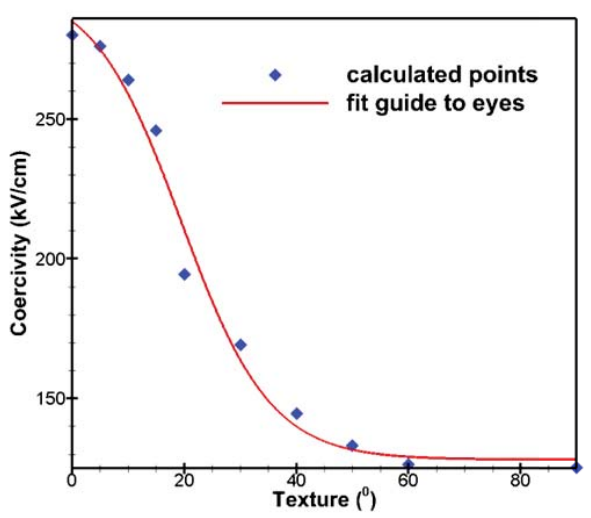

(b)

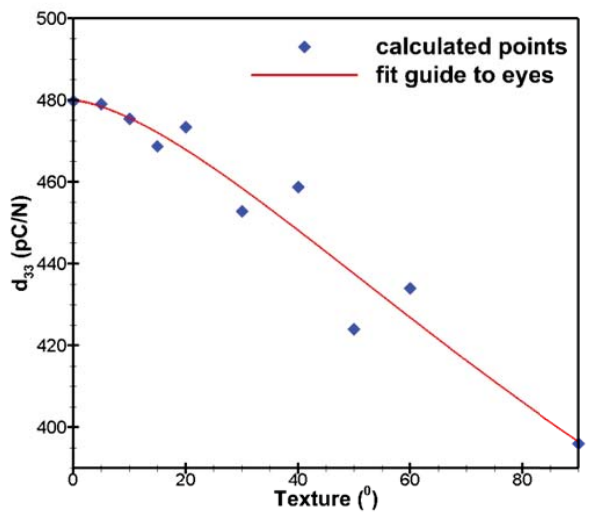

(d)

Figure 5.9 Extracted values from Fig. 5.8 for ferroelectric properties (a) Saturation polarization $\left(P_{S}\right)($ b) Coercivity (c) Dielectric constant (d) Piezoelectric coefficient vary with distribution angle. 


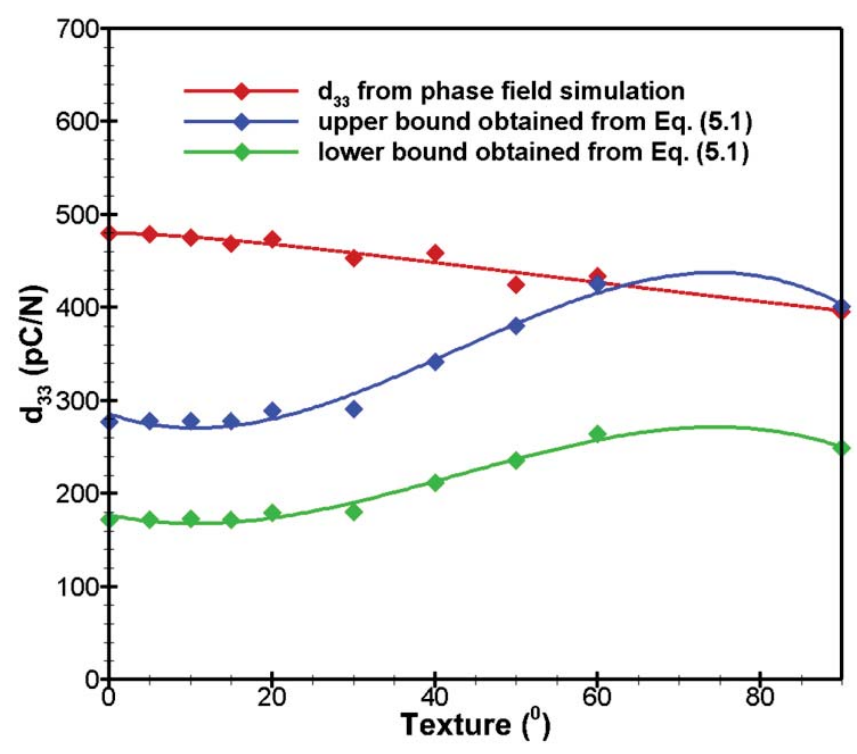

Figure 5.10 Red curve and points represent the phase field calculated piezoelectric coefficient $d_{33}$ with distribution angle, which is the same data set as shown in Fig. 5.9(d). Blue curve and points are obtained from Eq. (5.1) with $\bar{Q}_{11}=Q_{11}$ which determines upper bound for intrinsic values of $\mathrm{d}_{33}$. Green curve and points are obtained from Eq. (5.1) with $\bar{Q}_{11}=<Q_{11}>$ which determines lower bound for intrinsic values of $\mathrm{d}_{33}$.

\subsection{Conclusion}

In this work, shape and texture effects of ferroelectric polycrystals are studied through computer modeling and simulations. The results first show that grain shape effect contributes to ferroelectric properties via domain topology tailored by grain boundary density. The measured direction along lower grain boundary density better tolerates domain configuration with more domain variants, thus, grain shape of different aspect ratio takes effect on ferroelectric properties along the specific direction with different domain features due to the variant grain boundary density. Such shape effect is minor compared with grain texture. The investigation on texture effect is conducted through quantitatively 
constructing ferroelectric response loops with different distribution angle using phase field method. The resultant calculated curves show that remenance, coercivity, dielectric constant and piezoelectric coefficient all present non-linear monotonic behavior with distribution angle. Curves for remenance, coercivity, and dielectric constant show flattening feature above distribution angle $60^{\circ}$, while piezoelectric coefficient monotonically decreases with distribution angle without presenting such feature, which has been explained through the analogy to texture-property relation we studied before in TGG. Also, our calculation further confirms that the domain wall motion effect would significantly contribute to ferroelectric response. Fabrication of ferroelectric polycrystals to obtain the desired properties is crucial. Domain-level shape effect and grain orientation effect both supply the additional variants for tailoring materials performance, thus facilitate the improvements for ferroelectric properties. Phase field simulation provides a feasible way to quantitatively obtain microstructure-property relations in order to further guide manufacture process for desired materials property. 


\section{Chapter 6. Phase Field Modeling of Textured Ferroelectric Polycrystals: Dielectric and Piezoelectric Properties of Template-Matrix Composites ${ }^{* *}$}

As mentioned in previous Chapters, untextured polycrystalline materials with random grain orientations exhibit isotropic macroscopic properties, even though the properties of individual grains are generally anisotropic. To exploit anisotropic properties in polycrystalline materials, crystallographic texturing of grains is required [210]. Textured polycrystalline materials are economically attractive, which can offer properties comparable to single crystals but at much lower cost. To achieve texturing, special processing route is needed. In particular, uniaxial texture is obtained via templated grain growth (TGG) technique [38]. In textured ferroelectric polycrystals synthesized by TGG technique, well-oriented single-crystalline platelet seeds are purposely introduced in the green tape, which serve as templates for matrix grains to heterogeneously nucleate and epitaxially grow on their surfaces, consequently reorienting the matrix grains and inducing texture development. While templates of the same phase as the matrix grains are an ideal choice, single-crystalline templates of required shapes and sizes are not always available or cannot be economically fabricated, thus templates of different materials are usually used.

** The material contained in Chapter 6 will be submitted in Phase Field Modeling of Textured Ferroelectric Polycrystals. Part II: Dielectric and Piezoelectric Properties of Template-Matrix Composites, by Jie E. Zhou, Yu U. Wang. 
For example, barium titanate $\mathrm{BaTiO}_{3}$ (BTO) is used as templates to synthesize textured PMN-PT by TGG process $[\underline{125}, \underline{212}]$. In Chapter 4 , phase field model and computational diffraction method are employed to study grain structure evolution and texture development during TGG process; it is found that, while the degree of crystallographic texture increases with increasing template volume fraction, the average template seed distance also plays an important role, and reducing the template size and shortening the seed distance is an effective way to achieve higher texture at lower template volume fraction. In this Chapter, we develop a phase field model of polycrystalline ferroelectric composites to seamlessly incorporate the simulated textured grain structures and carry out computer simulation study of the relationships between grain structures, crystallographic textures, template seed distributions, domain processes and electromechanical properties in such textured ferroelectric polycrystals, with a particular focus on the competing effects of crystallographic texture and template seed volume fraction on the dielectric and piezoelectric properties. While increasing template volume fraction generally increases the degree of crystallographic texture, a higher volume fraction of templates is expected to cancel the advantage of texturing due to composite effect, because the template phase usually possesses material properties inferior to the matrix phase.

In this Chapter, phase field model of ferroelectric composites consisting of $\mathrm{BaTiO}_{3}$ (BTO) template seeds embedded in matrix grains of $\mathrm{Pb}\left(\mathrm{Zr}_{1-\mathrm{x}} \mathrm{Ti}_{\mathrm{x}}\right) \mathrm{O}_{3}(\mathrm{PZT})$ is developed to simulate domain evolution, polarization-electric field $(P-E)$ and strain-electric field $(\varepsilon-E)$ hysteresis loops for textured ferroelectric polycrystals. The coercivity, remanence, dielectric permittivity and piezoelectric coefficient are studied as functions of grain texture 
and template seed volume fraction. The composite effects of mechanical clamping and charge accumulation at inter-phase interfaces between matrix and template inclusions are analyzed.

\section{Model Preparation:}

Phase field models have been developed for composite materials in section 2.3 by combining our previous phase field models of ferroelectric polycrystals [108] and dielectric composites [213]. The model seamlessly incorporates the grain structures and textures as simulated by phase field modeling of TGG processes. The textured ferroelectric polycrystals consist of template-phase single-crystalline platelet seeds embedded in matrix-phase grains, as illustrated in Fig. 6.1. The grain structure of such a ferroelectric composite is characterized by a grain rotation matrix field $\mathbf{R}(\mathbf{r})$ that describes the geometry (size, shape, location) and crystallographic orientation of individual grains, as illustrated in Fig. 6.1(a). The two-phase morphology of the composite is characterized by a phase field variable $\eta(\mathbf{r})$ that distinguishes template seeds $(\eta=0)$ and matrix grains $(\eta=1)$, as illustrated in Fig. 6.1(b), where red and blue colors represent template and matrix phases, respectively. The state of such a ferroelectric polycrystal is described by a polarization field $\mathbf{P}(\mathbf{r})$, whose total system free energy under externally applied electric field $\mathbf{E}^{\mathrm{ex}}$ is from Eq. (2.2). Expansion coefficients and materials constants for two-phase system are conveniently considered via $\eta(\mathbf{r})$ in order to distinguish between two different phases (refer section 2.3 for detail). The evolutions of polarization and domain microstructure are characterized by the time-dependent Ginzburg-Landau equation from Eq. (2.4). In this 
Chapter, we consider PZT polycrystalline ceramics (composition $x=0.6$ at room temperature) textured by BT templates, whose material parameters have been experimentally determined [194, 195].

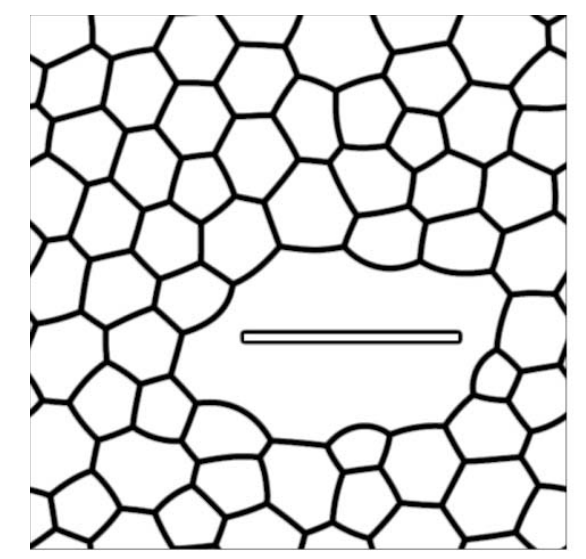

(a)

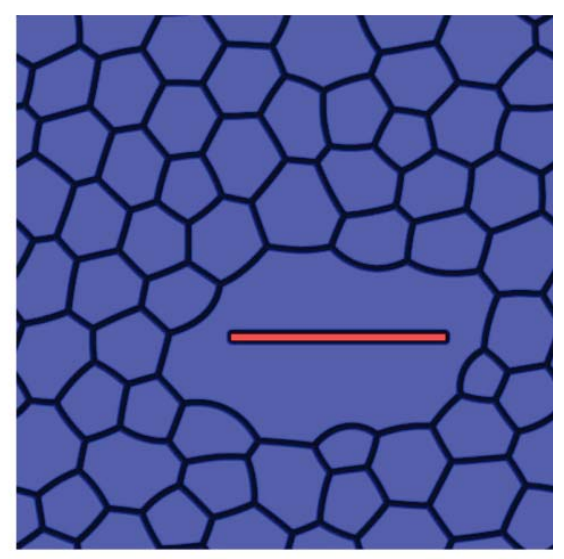

(b)

Figure 6.1 (a) Grain structure and (b) phase morphology of polycrystalline ferroelectric composite textured by templated grain growth.

\subsection{Texture Effects on Ferroelectric Properties in Two-Phase Composites}

Multi-domain configuration is common and important factor to exploit extrinsic effect for dielectric and piezoelectric properties in polycrystal materials. Multi-phase is an additional variant to further affect domain structure leading to more complex or entangled manner. Special behaviors of polarization acting on PZT and BTO phase boundaries should be purposely addressed. Fig. 6.2(a) shows the domain relaxation after quenching from above Curie temperature. Within each grain region, multi-domain structure commonly shows up due to the different grain orientations at boundary and long range electric-elastic interactions to further minimize total free energy. 


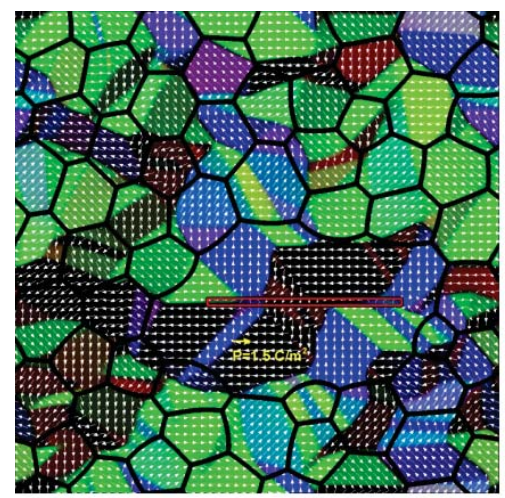

(a)

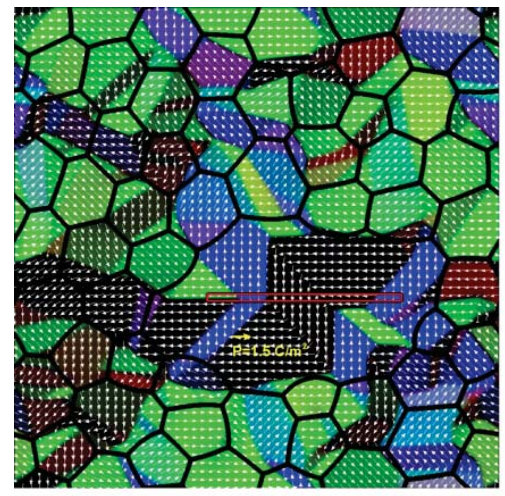

(c)

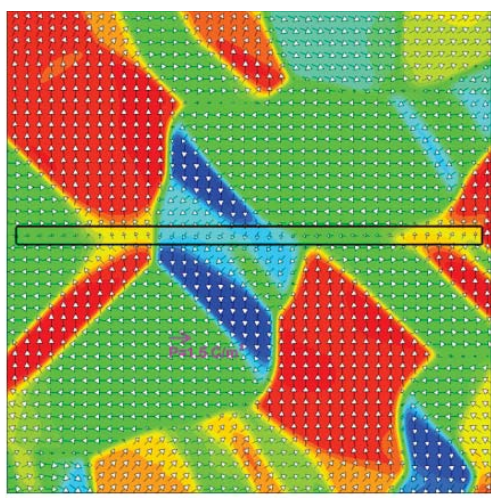

(b)

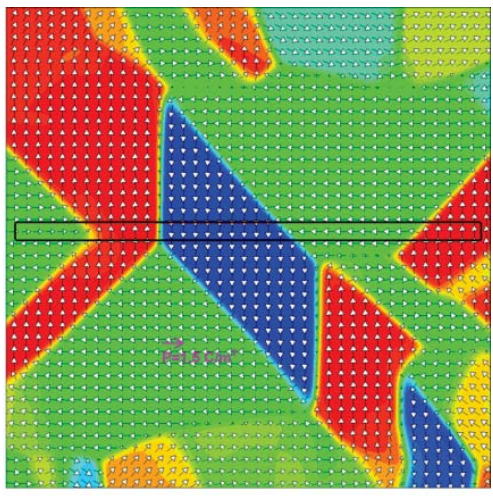

(d)

Figure 6.2 Simulated domain patterns quenching through Curie temperature: (a) two phase composite polycrystalline domains. (b) Zoom in portion of (a) close to template surface. (c) Pure PZT phase counterpart with the same grain structure and orientation as two phase composites. (d) Zoom in portion of (c) close to template surface. Solid black lines represent grain structure similar to Fig. 6.1 (a). White arrows designate in-plane polarization, while the reference polarization length is labeled with yellow and purple arrows and their magnitude. Red rectangular box is PZT-BTO phase boundary in (a) but is PZT-PZT phase boundary in (b). Alternatively contour map is visualized by $\mathrm{P}_{3}$ component out of plane in (b) and (d) instead of RGB in (a) and (c).

Usually, to eliminate electrostatic energy and elastostatic energy, twinning is the main feature for domain structure in polycrystals due to the existence of grain boundaries. In this section, we will focus on the domain structure occurring on the phase boundary to 
study its special configuration which potentially affects polarization switching properties. A detail portion for polarizations on the phase boundary is shown in Fig. 6.2(b).

Fig. 6.2(b) is the zoomed-in portion of Fig. 6.2(a) close to BTO minor phase boundary. Due to the existence of phase boundary, saturation properties for polarization on both sides are different. During domain relaxation, electrostatic energy always plays an important role [103], which means surface/bulk charges should be eliminated all the time. The configuration of domain inside template is tailored by domain structure adjacent to its surface in order to reduce electrostatic energy. Template itself acts as a transient zone to bridge the domains adjacent to upper and lower surfaces by keeping components of polarization continuous across the phase boundary to reduce surface charges. Fig. 6.2(b) also demonstrates that polarizations close to the template incline to align themselves along the larger dimensional surface because zero polarization component of surface normal satisfies the charge-free condition across the phase boundary, while other alignments more or less generate surface charges due to difference of saturation polarization between PZT and BTO. The second phase inclusions not only reinforce the forming of domain with polarization orientation along larger dimensional surface but also perform as selfaccommodating transient bridging zone to reduce electrostatic energy at the phase boundary during domain evolution process. For the comparison purpose, BTO template is substituted to PZT template with the same grain structure and orientation as shown in Fig. 6.2(c). Fig. 6.2(c) and (d) show the counterparts for Fig. 6.2(a) and (b) respectively by removing the effect of phase boundary circled by red box. Domain patterns in Fig. 6.2(c) and (d) demonstrate that polarization across the surface of red box is not affected by the 
invisible artificial boundary, where polarization "feels" no difference around. Therefore, two-phase boundary really affects the domain evolutions and further ferroelectric properties.

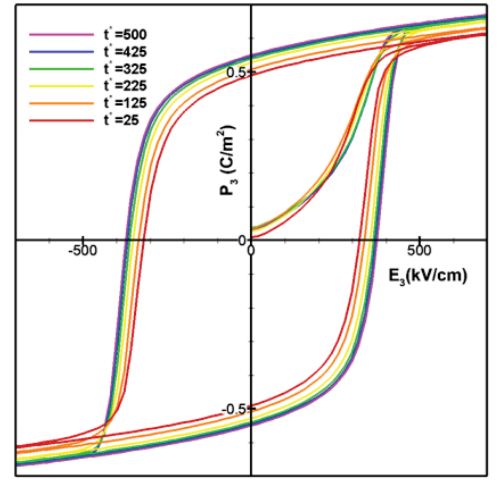

(a)

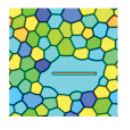

$t^{*}=25$

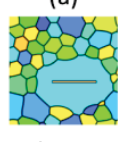

$\mathrm{t}^{*}=125$

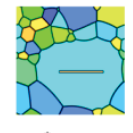

$\mathrm{t}^{*}=225$

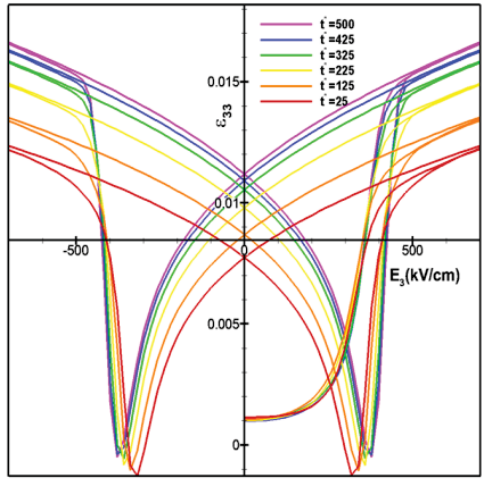

(b)

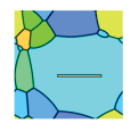

$t^{*}=325$
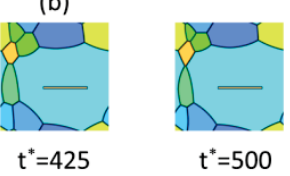

Figure 6.3 (a) E-P Loop (b) $\varepsilon$-E Loop at different reduced simulation time step $t^{*}=25,125$, $225,325,425,500$. The corresponding grain structures are shown at the bottom.

Fig. 6.3(a) and (b) summarize the time-dependent E-P Loop and $\varepsilon$-E Loop based on two-phase grain structures shown at the bottom. Different reduced simulation time step represents variant sintering time. Uniform electric field is applied along crystallographic axis [001] direction of template to obtain the system polarization and strain component also from the same direction. With time step increasing, both coercivity and remnance increase, so does $\varepsilon_{33}$ as the same manner. Such phenomenon attributes to the texture development when templated grains with well-defined uniaxial texture along [001] direction dominates more volume fraction of matrix. The assembly behavior approaches single crystal along [001] direction. Important ferroelectric properties such as remnance, coercivity, dielectric 
constant and piezoelectric coefficient with time evolution could be interpolated from Fig. 6.3 as shown in Fig. 6.4. Accompanied with the development of texture, all the properties start to show a monotonic trend towards the corresponding values of single crystal along [001] direction. It is worth noting that the slopes of Fig. 6.4(a)-(d) during the time evolution become smaller due to the slowing down of grain growth process close to the final stage.

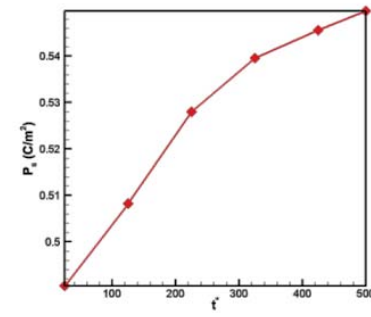

(a)

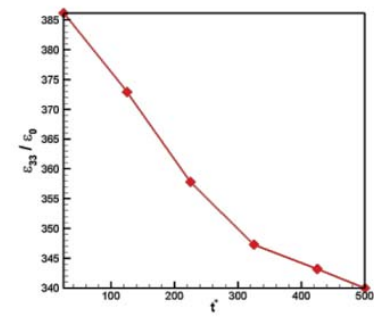

(c)

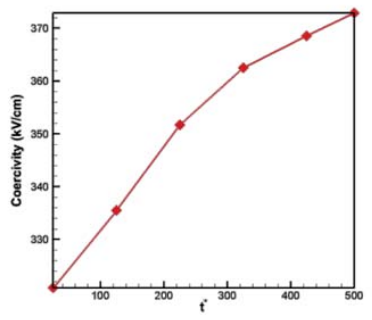

(b)

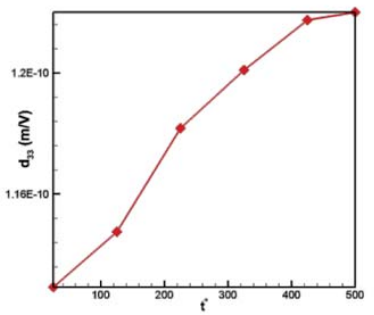

(d)

Figure 6.4 (a) Remnance (b) Coercivity (c) Dielectric Constant ( $\varepsilon_{33}$ ) (d) Piezoelectric Coefficient $\left(\mathrm{d}_{33}\right)$ with time evolution based on grain structure at bottom of Fig. 6.3.

To better understand the feature mechanisms of two phase composites polarization switching, a detail domain evolution snapshots near phase boundary are required as shown in Fig. 6.5. Domain pattern for different stage is labelled on the E-P Loop (leftmost graph in Fig. 6.5) for illustration purpose. Domain patterns of different stages for both two-phase composites (BTO template + PZT matrix $)$ and one-phase polycrystal (PZT template + PZT matrix) are shown together in the middle and rightmost columns of Fig. 6.5 respectively 
for comparison purpose. Fig. 6.5(a) (middle column) represents for domain pattern of the polarization saturation at the full strength of the applied electric field along [001] direction. Fig. 6.5(b) (middle column) shows remnant polarization state when external field strength reduces to zero.
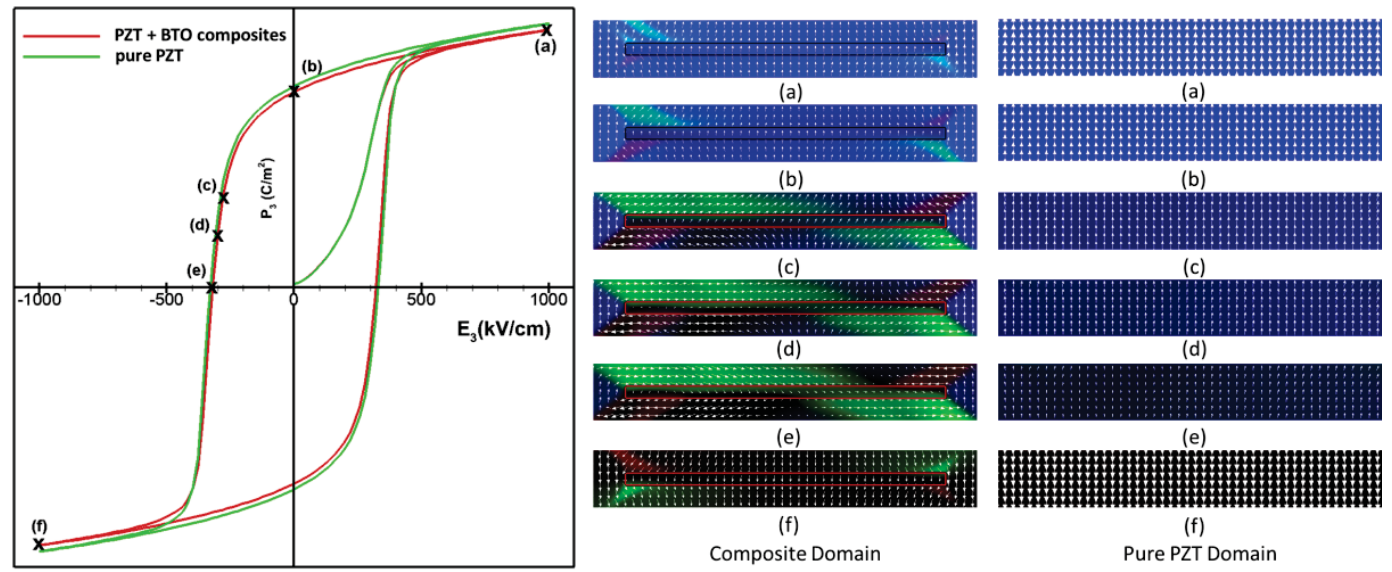

Figure 6.5 E-P curves (left) and snapshots of domain patterns close to template seed (right). (a)-(f) on the right side are selected area of domain patterns adjacent to phase boundary under different electric field strength and also designated on E-P curve (left) with bold symbol "X". The rightmost column represents domain patterns for pure PZT with the same grain structure and at the same loading stage as composites for comparison purpose. The E-P curve for pure PZT is also shown on the left with green color.

Compared with Fig. 6.5(a) (middle column), the length of polarization in Fig. 6.5(b) (middle column) is shorter and the direction of polarization rotates away from [001] by small angle because bulk free energy and grain orientations re-dominate domain structure. When the external field reverses to increase along the opposite direction [001], as shown in Fig. 6.5(c)-(e) (middle column), polarizations inside the BTO phase gradually rotate themselves towards the direction parallel to the surface, acting as an extra driving force to 
drag down the polarization in adjacent matrix of PZT in order to avoid generation of charges on the phase boundary. That coercivity of BTO is intrinsically smaller than PZT, supplies an embryo of thin layer for unstable polarization who wants to rotate ahead of time. In the purpose to decrease the surface charge, matrix polarizations are forced to rotate accordingly resulting in the tendency to switch early. In comparison, Fig. 6.5(a)-(e) (rightmost column) show normal domain switching behaviors of pure PZT phase without phase boundary effect.

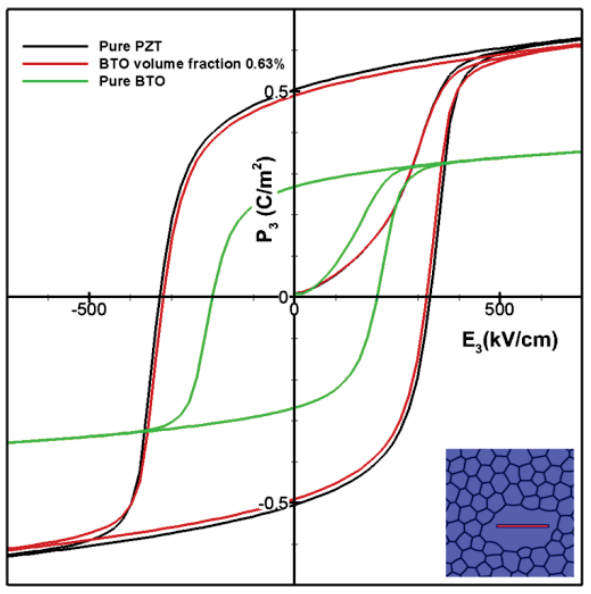

(a)

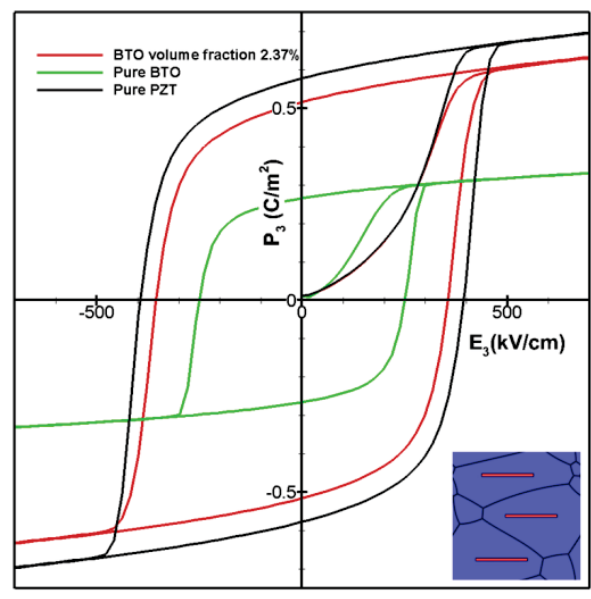

(b)

Figure 6.6 (a) E-P curves of pure PZT, pure BTO, and two phase composites with template volume fraction $0.63 \%$. (b) E-P curves of pure PZT, pure BTO, and two phase composites with template volume fraction $2.37 \%$. The insets on the corner of (a) and (b) show grain structures based on which E-P curves are calculated.

Such intrinsic interior property (lower coercivity) of BTO as an inclusion could affect the process of domain evolution acting as "early polarization reverse" habitants. In order to confirm such deduction, E-P curves of pure PZT and pure BTO systems with the same grain structure and texture as the two phase composites are calculated to make a 
comparison. Fig. 6.6(a) and (b) corresponding to different cases of BTO template volume fraction respectively further confirm such "composite effect" that the coercivity of two phase composites lays between pure PZT (high value) and pure BTO (low value) polycrystals. Volume fraction of templates plays a role to leverage the coercivity of composites as more BTO templates embedded in the matrix leads to the more sites of earlyreverse embryo, thus lower coercivity. Fig. 6.6(b) demonstrates such increasing softening of coercivity with higher volume fraction of BTO. So, the conclusion could be made that more volume fraction of templates will increase the composites effect, while as shown in Fig. 6.6(a), relatively lower template volume fraction, texture effect dominates.

\subsection{Competing Composites Effects on Dielectric and Piezoelectric Properties}

Effect of the average distance between seeds (seed number) plays an important role for texture development, therefore, results in various properties. Fig. 6.7 shows us the ferroelectric properties with different seed number at $t^{*}=300$ with fixed volume fraction to be $2.1 \%$. When seed number (average distance between seeds) increases (decreases), the resultant texture increase due to the higher chance for templated grains to contact each other by consuming adjacent smaller non-templated grains. In our model, template seeds are separated apart with relatively low second phase volume fraction (2.1\%), the effective ferroelectric properties due to second phase composites effect could be treated as fixed for different seed number cases by ignoring the factor from variant dispersion distribution based on the assumption of Maxwell model [214]. So, Fig. 6.7 presents the monotonic trend of different ferroelectric properties via texture development as the result of different 
seed number. Relative small distance between seeds results in higher texture, thus, better performance along textured direction.

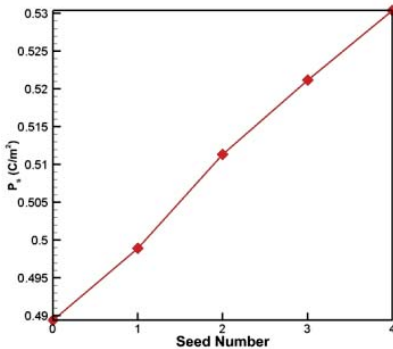

(a)

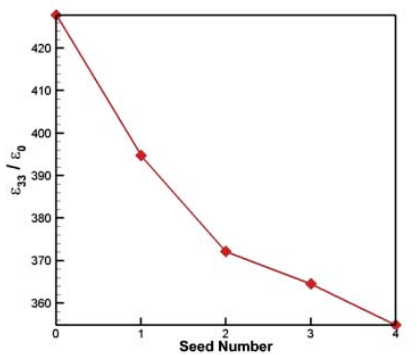

(c)

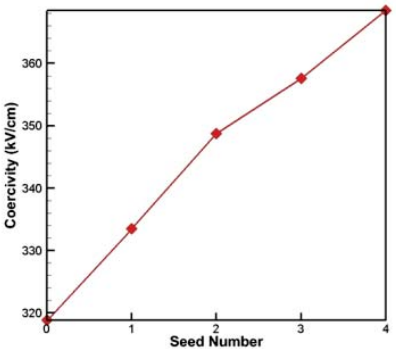

(b)

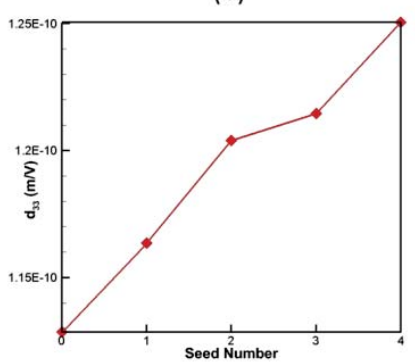

(d)
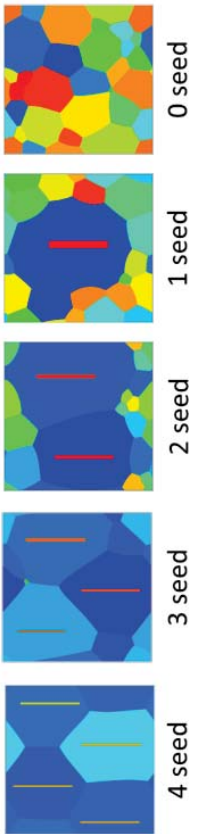

Figure 6.7 (a) Remnance (b) Coercivity (c) Dielectric Constant ( $\varepsilon_{33}$ ) (d) Piezoelectric Coefficient $\left(d_{33}\right)$ via seed number. The corresponding grain structures selected at $t^{*}=300$ with fixed seed volume fraction of $2.1 \%$ are shown on the right column.

Due to the industrial fabrication and requirement, each template seed usually has designated volume fraction and shape. More volume fraction commonly indicates more template seeds involved. Fig. 6.8(a) shows Lotgering factor development with different template volume fraction. The higher template volume fraction will facilitate Lotgering factor to approach to 1 faster. When template volume fraction exceeds $1 \%$, Lotgering factor presents the feature of saturation behavior without much improvement on texture by adding more template volume fraction as shown in Fig. 6.8(a). Figs. 6.8(b), (c) and (e) show the properties "drop" when "composites effect" takes lead, while texture already approaches 
its saturation. Remnance, coercivity and piezoelectric constant show the similar trend via template volume fraction where a predictable maximum appears around $1 \%$ volume fraction. It is shown that a competing mechanisms exist between texture and minor inferior second phase. On the one hand, more volume fractions of second phase could improve texture leading to more enhanced anisotropic properties, on the other hand, however, more volume of inferior second phase could also depress ferroelectric performance. When volume fraction approaches to certain extent, texture will not be improved anymore, resulting in worse ferroelectric response if more templates participate. The existence of critical point further demonstrates the competing mechanisms. Fig. 6.8(d) shows the opposite trend in value of dielectric constant $\varepsilon_{33}$ which takes the minimum value around $1 \%$ volume fraction, while, inserting more templates only increases $\varepsilon_{33}$ slightly. Based on Eq. (5.1) we discussed in Chapter 5, upper bound and lower bound for $\mathrm{d}_{33}$ with seed volume fraction are also calculated from such intrinsic relation. For the calculation, $Q_{11}=0.03864$ $\mathrm{m}^{4} / \mathrm{C}^{2}, Q_{12}=-0.0184 \mathrm{~m}^{4} / \mathrm{C}^{2}$ and $Q_{44}=0.0819 \mathrm{~m}^{4} / \mathrm{C}^{2}$ are adopted. $<Q_{11}>$ is then calculated to be $0.032204 \mathrm{~m}^{4} / \mathrm{C}^{2}$ for lower bound. Fig. 6.9 shows the upper bound, lower bound and calculated $d_{33}$ from phase field simulation all together. We could recall that the calculated $d_{33}$ curve from phase field shown in Fig. 5.1 in previous chapter is not sandwiched by upper and lower bounds due to the domain wall motion effect. However, as shown in Fig. 6.9, calculated $d_{33}$ curve with seed volume fraction is generally sandwiched by the two bound curves. This phenomenon could be explained by the relatively strong domain wall pinning effect in the two-phase composites, which reduces the domain wall motion effect resulting in the matching on $d_{33}$ magnitude predicted by bound curves. It is worth to mention that 
although the magnitude predicted from intrinsic relation is reasonable, the trend could not be correctly reproduced merely by Eq. (5.1) because some kinetic features are ignored by such simplified intrinsic relation equation. Except for domain wall motion effect, twophase interfaces and arrangements may additionally contribute to the mismatch between predicted values by Eq. (5.1) and phase field simulation, which necessitates the numerical simulations with phase field.

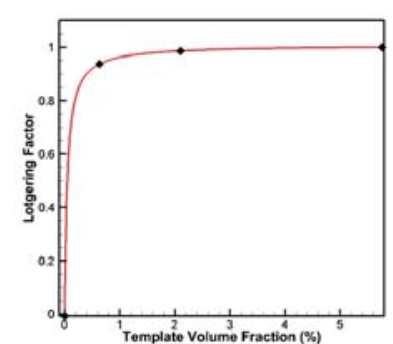

(a)

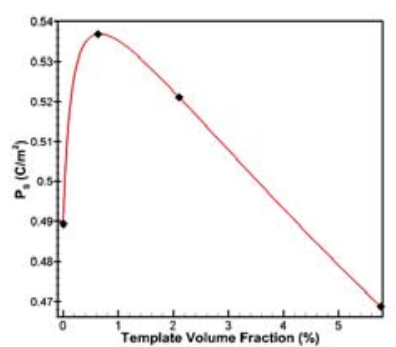

(b)

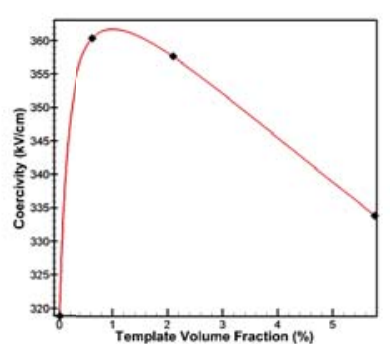

(c)

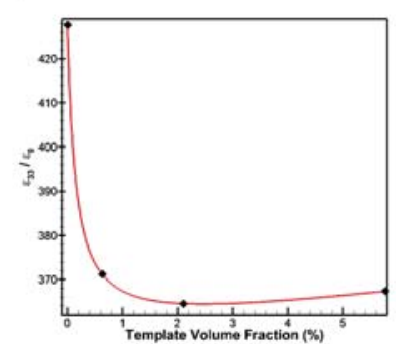

(d)

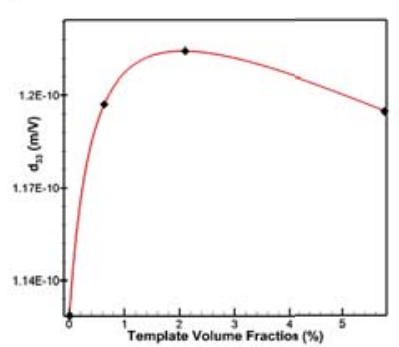

(e)
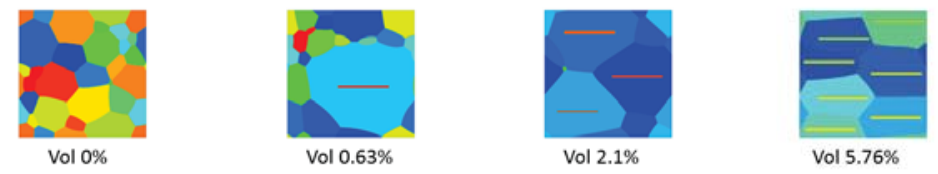

Figure 6.8 (a) Lotgering Factor (b) Remnance (c) Coercivity (d) Dielectric Constant ( $\left.\varepsilon_{33}\right)$ (e) Piezoelectric Coefficient $\left(\mathrm{d}_{33}\right)$ via seed volume fraction. The corresponding grain structures selected at $t^{*}=300$ with various seed volume fractions are shown at the bottom row. 


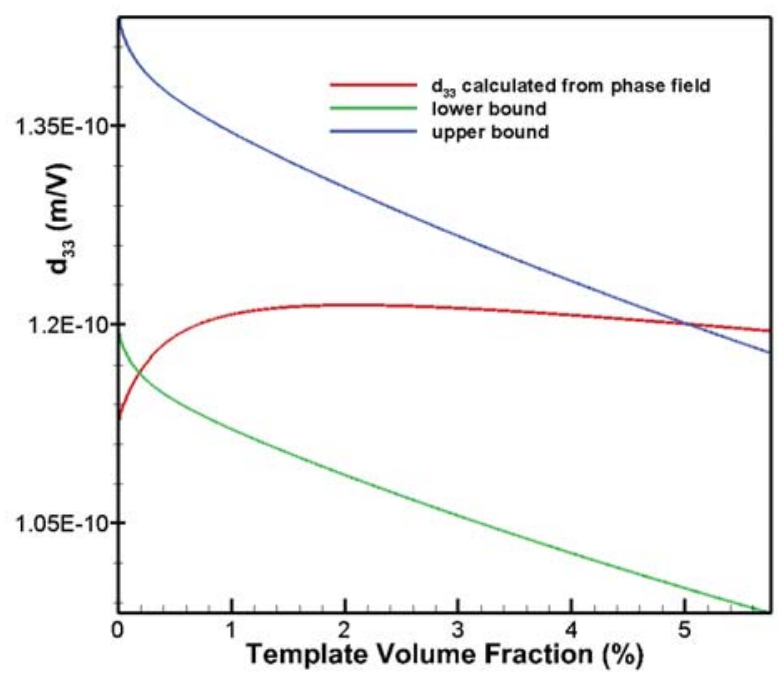

Figure 6.9 Piezoelectric Coefficient $\left(\mathrm{d}_{33}\right)$ via seed volume fraction (red curve). The upper bound (blue curve) obtained from Eq. (5.1) with $\bar{Q}_{11}=Q_{11}$. The lower bound (green curve) obtained from Eq. (5.1) with $\bar{Q}_{11}=<Q_{11}>$.

\subsection{Conclusion}

Pre-designed phase field simulation is performed to investigate the relations between ferroelectric properties and grain texture in the coherent ferroelectric two phase composites. Simulation shows that during templated grain growth process, ferroelectric properties monotonically obtain the enhancement accompanied with the development of texture. Also, by fixing the template volume fraction, the template seed number (average distance between template seeds) against ferroelectric properties are calculated showing the trend that enhanced properties are obtained at higher template seed number due to the ability to form the higher texture. As a particular feature of two phase composites, domain kinetics at the phase boundaries are studied in detail during the domain switching. Polarization across interphase boundary inclines to significantly eliminate electrostatic 
energy resulting in the specific bridging domain structure at the interphase boundary. Moreover, such bridge domain mechanisms link the surface polarizations of both phases in a correlated way that the involvement of lower coercivity materials causes polarization at the phase boundary "easier to switch down" thus resulting in lower coercivity compared with primary single phase materials. As expected, texture effect and volume fraction of second inferior phase compete on the performance with each other. Such tug of war mechanism results in the critical point around 1\% BTO template volume fraction with the best properties which could be explained by the saturation of Lotgering factor when further BTO templates are embedded in. This competing detrimental effect is found to arise from the composite effect, where the template phase possesses material properties inferior to the matrix phase, causing mechanical clamping and charge accumulation at inter-phase interfaces between matrix and template inclusions. Finally, the phase field simulation offers the prediction of the best cost-performance ratio for two phase ferroelectric composites facilitating the industrial fabrication. Again, we confirmed that simplified intrinsic relation equation could not well reproduce $\mathrm{d}_{33}$ value by ignoring domain wall motion and two-phase arrangements. 


\section{Chapter 7. Diffraction Analysis of Lithium Ion Battery Cathode Materials ${ }^{\dagger \dagger}$}

Novel fabrication especially with the focus on tailoring ion compositions [148] to further improve lithium ion battery cathode paves a way for new discoveries of high performance materials such as pristine Toda HE5050 (TODA America, Inc. and Argonne National Lab) with the nominal composition form $\mathrm{Li}_{1.2} \mathrm{Ni}_{0.15} \mathrm{Mn}_{0.55} \mathrm{Co}_{0.1} \mathrm{O}_{2}$. Recent experimental work [215] based on its structural changes during cycling is presented with selected electron diffraction and TEM. In particular, Toda HE5050 could be reformulated as $0.5 \mathrm{Li}_{2} \mathrm{MnO}_{3}-0.5 \mathrm{Li}\left(\mathrm{Ni}_{0.375} \mathrm{Co}_{0.25} \mathrm{Mn}_{0.375}\right) \mathrm{O}_{2}$ in purpose to distinguish with solid solution notation. Based on the Rietveld refinement of high-resolution synchrotron X-ray powder diffraction data of pristine Toda HE5050, DIFFaX software [198] is exploited to perform computational analysis to gain quantitative insight into the structural characteristics in regard to its nature of solid solution versus two-phase intergrowth. Furthermore, as a good prototypical both for the investigations of structural characteristics and in-layer ordering, innovative Toda HE5050 deserves theoretical studies in depth serving as a basis for subsequent study of the structural changes during charge-discharge cycles as related to lithium extraction/insertion and capacity fade phenomenon of the cathode materials. In order to prepare a useful tool to better investigate structural information for Toda HE5050, firstly, a hierarchical structural refinement algorithm combined with $\mathrm{DIFFaX}$ is developed

${ }^{*}$ Part of the material contained in Chapter 7 will be submitted in Analysis of $x \mathrm{LiCoO}_{2}-(1$ x) $\mathrm{Li}_{2} \mathrm{MnO}_{3}$ Lithium Ion Battery Cathode Materials: A Hierarchical Structural Refinement Approach, by Jie E. Zhou, Yu U. Wang. 
to quantitatively study the stacking faults in the structural similar but less complex system as $x \mathrm{LiCoO}_{2} \cdot(1-x) \mathrm{Li}_{2} \mathrm{MnO}_{3}$ with the availability of a range of experimental data corresponding to different composition $\boldsymbol{x}$. Such analysis aims at linking the composition of high performance with certain stacking faults distribution. Then, such proposed strategy/algorithm is applied to Toda HE5050 to obtain some useful structural features. At last, to further extend the ability of our present models, Oxygen layer faulting structural model and the way to involve voids in DIFFaX are proposed to explain the peculiar behaviors caused by potential Oxygen layer faulting mechanism and to offer a possible way to study the dynamic process of lithium extraction/insertion respectively.

\subsection{Analysis of $x \mathrm{LiCoO}_{2}-(1-x) \mathrm{Li}_{2} \mathrm{MnO}_{3}$ Lithium Ion Battery Cathode Materials: A Hierarchical Structural Refinement Approach}

A hierarchical structural refinement approach is developed to analyze the powder diffraction data of layered $x \mathrm{LiCoO}_{2}-(1-x) \mathrm{Li}_{2} \mathrm{MnO}_{3}$ lithium ion battery cathode materials in this section. The method employs three-stage sequential fittings via seamless integration of Rietveld refinement, DIFFaX simulation and structural heterogeneity (stacking fault) refinement. The third-stage fitting is carried out based upon the database constructed using $\mathrm{DIFFaX}$ from the Rietveld refinement results of experimental diffraction data. The approach is applied to investigate the stacking faults in $x \mathrm{LiCoO}_{2}-(1-x) \mathrm{Li}_{2} \mathrm{MnO}_{3}$ and the dependence on composition $x$. The two-phase intergrowth model with stacking faults are found to be more realistic with better fitting to the experimental data than the solid solution model for all compositions except for pure $\mathrm{LiCoO}_{2}(x=1)$ where the two models become 
identical. It is found that introduction of $\mathrm{LiCoO}_{2}$ phase reduces the stacking faults in the $\mathrm{Li}_{2} \mathrm{MnO}_{3}$ phase. For $0.5 \mathrm{LiCoO}_{2}-0.5 \mathrm{Li}_{2} \mathrm{MnO}_{3}(x=0.5)$, the analysis reveals special $\mathrm{Li}_{2} \mathrm{MnO}_{3}$ structures of ordered stacking superlattice and alternative perfect stacking that coexist with the conventional $\mathrm{Li}_{2} \mathrm{MnO}_{3}$ phase with stacking faults, which suggests a structural origin of the improved electrochemical performance at this composition. The method has the potential to incorporate more structural phases involved in electrochemical processes to study structural changes during charge-discharge cycles as related to lithium ion extraction/insertion of the battery materials.

\subsubsection{Introduction}

Lithium ion battery cathode materials have layered structures, which exhibit chemical disorders associated with ion exchanges and stacking faults associated with the ordered (or partially ordered) metal layers. Determination of the crystal structures and the structural defects is important for understanding the electrochemical processes and improving the battery performance of the cathode materials.

This work is motivated by the limitation in the current diffraction analysis method to extract information of stacking faults. Rietveld refinement is a powerful method to determine crystal structures from experimental powder diffraction data. In this method, a unit cell is used to define the crystal structure, including lattice parameters, atomic coordinates, site occupancies, and thermal displacements. Chemical disorders associated with atomic exchanges are characterized by site occupancies within the unit cell. These structural variables are refined against powder diffraction intensity profiles to get the best fitting, which determines the crystal structure. However, limited by the use of the unit cell, 128 
Rietveld refinement cannot determine the crystal structure with stacking faults; instead, the effects of stacking faults are averaged into the unit cell and described as chemical disorders. Since stacking faults are prominent features of lithium ion battery cathode materials, it is desirable to extract quantitative information of stacking faults. In this work, we demonstrate the capability of the developed hierarchical refinement approach to structural analysis of $x \mathrm{LiCoO}_{2}-(1-x) \mathrm{Li}_{2} \mathrm{MnO}_{3}$ with stacking faults. The methodology can be further developed to incorporate more layered phases thus has the potential to study the structural changes during charge-discharge cycles as related to lithium ion extraction/insertion and capacity fade phenomenon of the battery cathode materials. Fig. 7.1 illustrates the hierarchical structural refinement strategy of multi-level sequential fittings, which integrates three computational methods, namely, Rietveld refinement, DIFFaX simulation, and structural heterogeneity (stacking fault) refinement, as discussed in the following.

\subsubsection{Methodology}

The hierarchical structural refinement approach is implemented in the following three stages of sequential fittings.

(I) Solid solution model with ion exchanges (Rietveld refinement): As the first stage to perform the hierarchical structural refinement, high-resolution synchrotron X-ray powder diffraction data of $y \mathrm{LiCoO}_{2}-(1-y) \mathrm{Li}\left(\mathrm{Li}_{1 / 3} \mathrm{Mn}_{2 / 3}\right) \mathrm{O}_{2}$ (where $y=2 x /(3-x)$ ) are analyzed by Rietveld refinement using GSAS to obtain the structural information with the following solid solution model with $\mathrm{Li}$ and $\mathrm{Mn}$ exchanges between $2 \mathrm{~b}$ and $4 \mathrm{~g}$ sites of the metal layers:

$\mathrm{Li}^{2 c} \mathrm{Li}^{4 \mathrm{~h}}\left[\left(\mathrm{Li}_{1-y-2 z} \mathrm{Co}_{y} \mathrm{Mn}_{2 z}\right)^{2 \mathrm{~b}}\left(\mathrm{Li}_{z} \mathrm{Co}_{y} \mathrm{Mn}_{1-y-z}\right)^{4 \mathrm{~g}}\right] \mathrm{O}^{4 \mathrm{i}} \mathrm{O}^{8 \mathrm{j}}$, 
Diffraction data of 7 compositions, namely $x=0,0.1,0.3,0.5,0.7,0.9,1.0$ (corresponding to $y=0,0.069,0.222,0.4,0.609,0.857,1.0)$, are analyzed as solid solutions. The structural parameters for each composition $y$ are obtained, including monoclinic lattice parameters $(a, b, c, \beta)$, fractional coordinates $\left(x_{\text {rel }}, y_{\text {rel }}, z_{\text {rel }}\right)$ and isotropic Debye-Waller factor $\left(B_{\text {iso }}\right)$ of each atomic site, and site occupancies (in terms of $y$ and $z$ ). To integrate seamlessly with DIFFaX in the second stage, Gaussian instrumental broadening $(u, v, w)$ is used, while strain broadening and other parameters that are not supported in DIFFaX are turned off. The treatment for the purpose of calculating high resolution diffraction in DIFFaX and the convention between broadening parameters of DIFFaX and GSAS could be found in Appendix B.

\section{Hierarchical Structural Refinement}

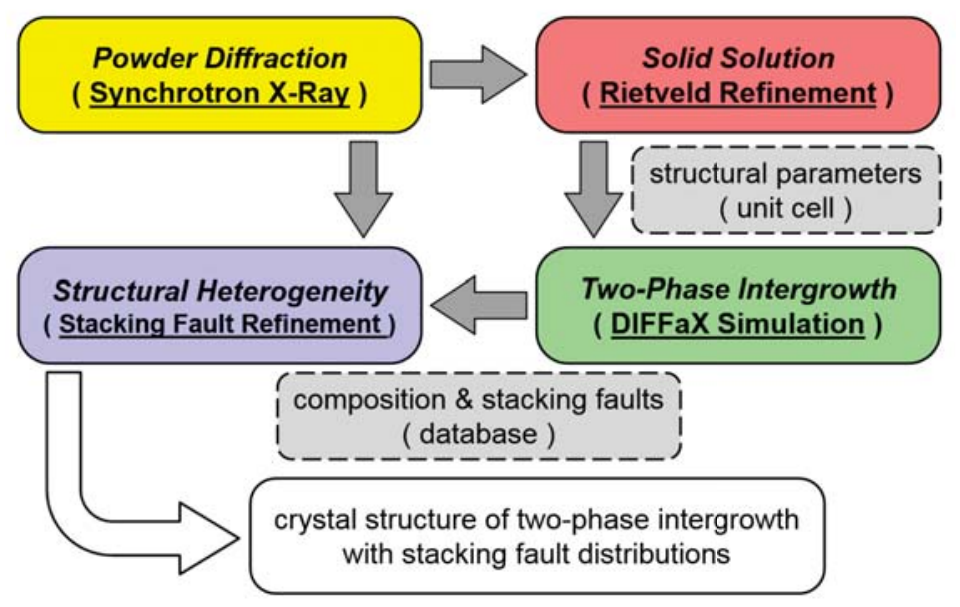

Figure 7.1 Schematic illustration of a hierarchical refinement strategy for structural analysis of layered two-phase intergrowth materials with stacking faults by integrating synchrotron X-ray powder diffraction, Rietveld refinement, DIFFaX simulation, and structural heterogeneity (stacking fault) refinement. 
(II) Two-phase intergrowth model with stacking faults (DIFFaX simulation): In this second stage to perform the hierarchical structural refinement, DIFFaX simulations are carried out based on Rietveld refinement results obtained in the first stage. A database of powder diffraction intensity profiles for different compositions $x$ (or equivalently $y$ ) with varying stacking fault probabilities (SFPs) are constructed using the following two-phase intergrowth model as illustrated in Fig. 7.2:

$y \mathrm{Li}^{2 \mathrm{c}} \mathrm{Li}^{4 \mathrm{~h}}\left(\mathrm{Co}^{2 \mathrm{~b}} \mathrm{Co}^{4 \mathrm{~g}}\right) \mathrm{O}^{4 \mathrm{i}} \mathrm{O}^{8 \mathrm{j}}-(1-y) \mathrm{Li}^{2 \mathrm{c}} \mathrm{Li}^{4 \mathrm{~h}}\left(\mathrm{Li}^{2 \mathrm{~b}} \mathrm{Mn}^{4 \mathrm{~g}}\right) \mathrm{O}^{4 \mathrm{i}} \mathrm{O}^{8 \mathrm{j}}$,

It is worth noting that the two-phase intergrowth model is built upon the two perfectly ordered end members $\mathrm{LiCoO}_{2}$ and $\mathrm{Li}_{2} \mathrm{MnO}_{3}$ without chemical disorder (there is no ion exchange between $\mathrm{Li}$ and $\mathrm{Mn}$, i.e., $z=0$ ), as illustrated in Fig. 7.2, where there is no stacking fault for $\mathrm{LiCoO}_{2}$ phase while $\mathrm{Li}_{2} \mathrm{MnO}_{3}$ phase may exhibit stacking fault probability ranging from $0 \%$ to $100 \%$. To simulate the powder diffraction intensity profiles for each of the 7 compositions $(y=0,0.069,0.222,0.4,0.609,0.857,1.0)$, the lattice parameters $a, b, c, \beta$ and Gaussian instrumental broadening parameters $u, v, w$ as obtained by Rietveld refinement for the same composition are used, while the fractional coordinates $x_{\text {rel }}, y_{\text {rel }}, z_{\text {rel }}$ and isotropic Debye-Waller factor $B_{\text {iso }}$ of each atomic site as determined by Rietveld refinement for the two perfectly ordered end members are adopted, and no $\mathrm{Li}$ and $\mathrm{Mn}$ exchange between $2 \mathrm{~b}$ and $4 \mathrm{~g}$ sites of the metal layers $(z=0)$ is assumed. Stacking fault probabilities ( $\eta$ for convenience) of $\mathrm{Li}_{2} \mathrm{MnO}_{3}$ phase between $0 \%$ and $100 \%$ with $10 \%$ increment are simulated (i.e., $\eta=0,0.1,0.2,0.3,0.4,0.5,0.6,0.7,0.8,0.9,1.0$ ). Thus 11 powder diffraction intensity profiles $I_{\eta}(2 \theta)$ are constructed for each composition except 
for pure $\mathrm{LiCoO}_{2}$ phase (which does not exhibit stacking faults thus has only one intensity profile in the database), yielding a total of 56 intensity profiles in the whole database.

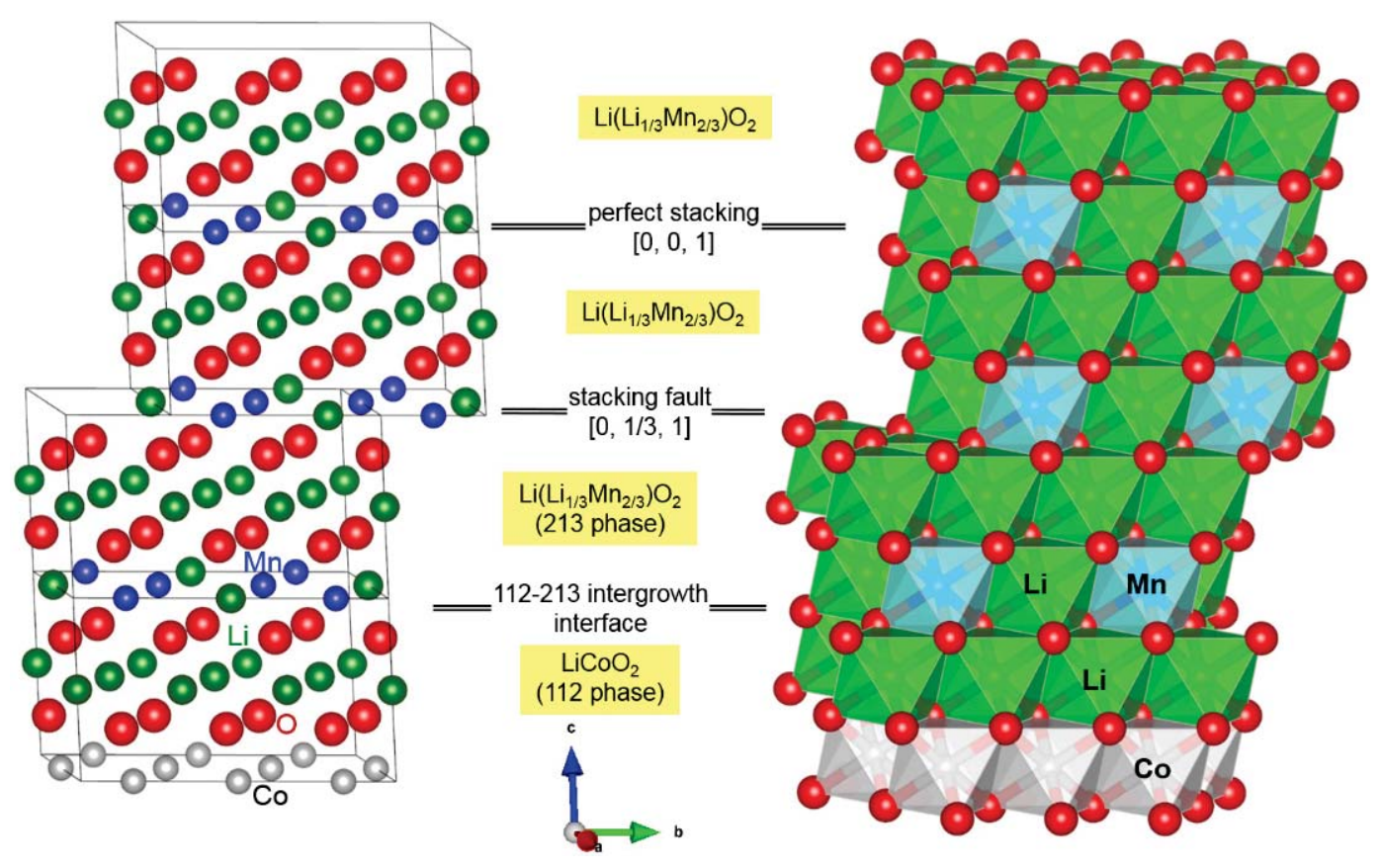

Figure 7.2 Illustration of two-phase intergrowth model of $\mathrm{LiCoO}_{2}-\mathrm{Li}_{2} \mathrm{MnO}_{3}$ with stacking faults, as visualized using VESTA. The unit cells of $\mathrm{LiCoO}_{2}$ and $\mathrm{Li}\left(\mathrm{Li}_{1 / 3} \mathrm{Mn}_{2 / 3}\right) \mathrm{O}_{2}$ phase layers, stacking sequences, stacking faults in $\mathrm{Li}\left(\mathrm{Li}_{1 / 3} \mathrm{Mn}_{2 / 3}\right) \mathrm{O}_{2}$ phase, and two-phase intergrowth are implemented in DIFFaX to simulate powder diffraction intensity profiles.

(III) Stacking fault distribution refinement: Based on the database constructed by DIFFaX in the second stage, stacking fault distributions are determined for each composition by fitting to the experimental powder diffraction data, which provide quantitative information about the spatially heterogeneous distributions of the stacking faults. To carry out this final stage refinement for each composition, the following fitting procedure is employed: 
$R_{P}=\frac{\int\left|\sum_{\eta} \omega_{\eta}^{2} I_{\eta}(2 \theta)+I_{\mathrm{b}}(2 \theta)-\kappa I_{\text {exp }}(2 \theta)\right| d(2 \theta)}{\int\left|I_{\text {exp }}(2 \theta)\right| d(2 \theta)}$,

where $I_{\exp }(2 \theta)$ is the experimental powder diffraction intensity profile for the composition, $I_{\mathrm{b}}(2 \theta)$ is a fitting background intensity distribution, $\kappa$ is an intensity scaling factor, and $\omega_{\eta}$ is a parameter to characterize the contribution of intensity profile $I_{\eta}(2 \theta)$ to the total intensity profile (the use of $\omega_{\eta}^{2}$ instead of $\omega_{\eta}$ in Eq. 7.3 automatically excludes the unphysical negative contribution). The stacking fault distribution in $\mathrm{Li}_{2} \mathrm{MnO}_{3}$ phase is described by the SFP weight (volume fraction) parameters $w_{\eta}=\omega_{\eta}^{2} / \sum_{\eta} \omega_{\eta}^{2}$. It is worth noting that the refinement is carried out by minimizing $R_{P}$ residual error parameter rather than $R_{W}$ parameter, because $R_{P}$ is more suitable for fitting the relatively weak superlattice reflection intensities associated with stacking faults while $R_{W}$ emphasizes the strong Bragg peaks but suppresses the sensitivity to the weak superlattice reflections. In this work, a linear background intensity function $I_{\mathrm{b}}(2 \theta)=C_{0}+C_{1}(2 \theta)$ is used (nevertheless, the method is applicable to more general background functional form). To search for the parameters $\omega_{\eta}, C_{0}, C_{1}$ and $\kappa$ that provide the best fitting to the experimental data, a simulated annealing algorithm is employed to minimize $R_{P}$ defined in Eq. 7.3, and the normalization condition $\sum_{\eta} \omega_{\eta}^{2}=1$ maintained during the refinement. Simulated annealing algorithm has the advantages to deal with non-linear non-analytical function (such as $R_{P}$ ) in multi-dimensional search space (such as 14 fitting parameters here). More detail 
information on the treatment to obtain the minimized $R_{P}$ values could be found in Appendix C.

In the following, the above described hierarchical structural refinement method of multi-level sequential fittings is applied to analyze the high-resolution synchrotron X-ray powder diffraction data of $x \mathrm{LiCoO}_{2}-(1-x) \mathrm{Li}_{2} \mathrm{MnO}_{3}$ lithium ion battery cathode materials, and the results are presented and discussed.

\subsubsection{Distributions of Stacking Faults as Function of Composition}

Fig. 7.3 compares the fitting residual error $R_{P}$ after each stage of the hierarchical structural refinement for different compositions. It is clearly seen that the two-phase intergrowth model with stacking faults has significantly smaller $R_{P}$ than the solid solution model. The difference between the two models is the largest for pure $\mathrm{Li}_{2} \mathrm{MnO}_{3}$ phase $(x=0)$ and decreases with increasing composition $x$, which is associated with the decrease in $\mathrm{Li}_{2} \mathrm{MnO}_{3}$ content and increase in $\mathrm{LiCoO}_{2}$ content. The two models become identical for pure $\mathrm{LiCoO}_{2}(x=1.0)$ which does not exhibit any stacking faults. As shown in Figs. 7.47.12, the noises in the experimental data contribute to the residual error $R_{P}$ even when good fitting is achieved. Nevertheless, comparison of the fitting results unambiguously shows that two-phase intergrowth is a more realistic model for $x \mathrm{LiCoO}_{2}-(1-x) \mathrm{Li}_{2} \mathrm{MnO}_{3}$ lithium ion battery cathode materials. The stacking faults are a general structural feature of this layered material system, which shuffle the metal layers of $\mathrm{Li}_{2} \mathrm{MnO}_{3}$ phase (while each layer is assumed to be perfectly ordered in the two-phase intergrowth model). When analyzed using Rietveld refinement, the shuffling of metal layers due to stacking faults are 
manifested as chemical disorders (ion exchanges between $2 \mathrm{~b}$ and $4 \mathrm{~g}$ sites of the metal layer) within the unit cell.

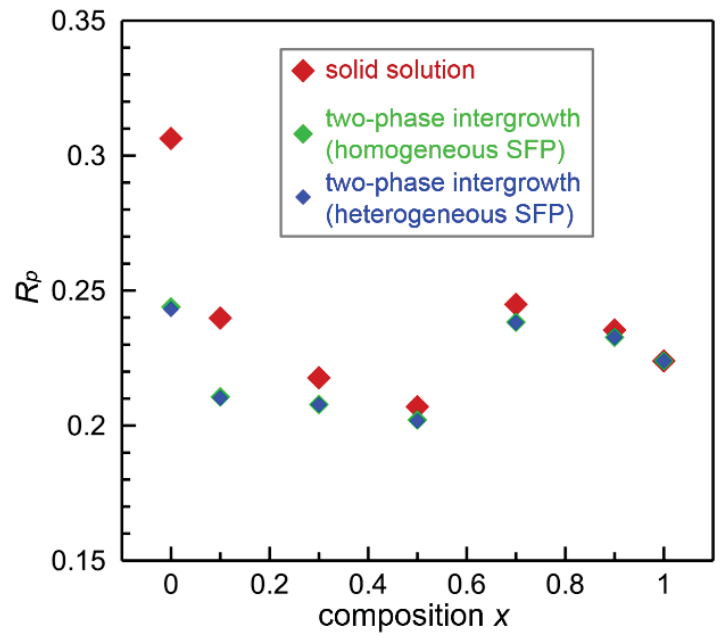

Figure 7.3 Comparison of fitting residual error $R_{P}$ among solid solution model, two-phase intergrowth model with homogeneous and spatially heterogeneous distributions of stacking faults.

Fig. 7.3 also shows that the fitting can be further improved by considering spatially heterogeneous distributions of stacking faults rather than assuming homogeneous distribution of stacking faults, as discussed in detail in the following. Again, the improvement in fitting is more prominent for lower composition $x$ where $\mathrm{Li}_{2} \mathrm{MnO}_{3}$ content is higher. The stacking fault distributions in $\mathrm{Li}_{2} \mathrm{MnO}_{3}$ phase of $x \mathrm{LiCoO}_{2}-(1-x) \mathrm{Li}_{2} \mathrm{MnO}_{3}$ for different compositions $x$ are shown in Figs. 7.4-7.12, where it is observed that the stacking fault probability in $\mathrm{Li}_{2} \mathrm{MnO}_{3}$ phase is the highest in pure $\mathrm{Li}_{2} \mathrm{MnO}_{3}(x=0)$ and decreases with increasing $\mathrm{LiCoO}_{2}$ content. 

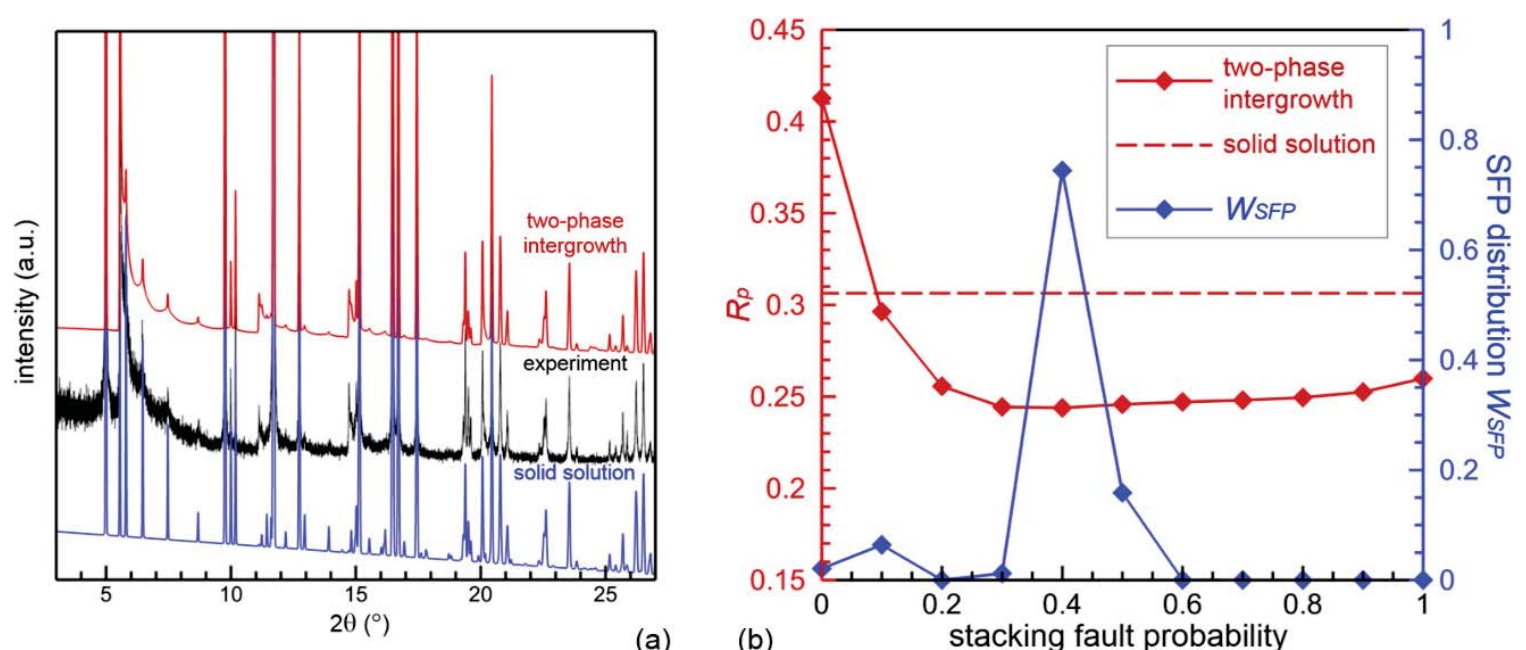

(a)

(b)

Figure 7.4 Comparison of two-phase intergrowth model with stacking faults and solid solution model for pure $\mathrm{Li}_{2} \mathrm{MnO}_{3}(x=0)$. (a) Powder diffraction intensity profiles of experiment, solid solution model, and two-phase intergrowth model with heterogeneous distributions of stacking faults. (b) Fitting residual error $R_{P}$ of solid solution model and two-phase intergrowth model with different homogeneous stacking fault probabilities. Best fitted spatially heterogeneous distributions of stacking faults are also shown in (b).

Fig. 7.4 compares the refinement results of two-phase intergrowth model with stacking faults and solid solution model for pure $\mathrm{Li}_{2} \mathrm{MnO}_{3}(x=0)$. Fig. 7.4 (a) shows the powder diffraction intensity profiles of experiment, solid solution model, and two-phase intergrowth model with the heterogeneous distributions of stacking faults as shown in Fig. 7.4(b). It is clearly seen that stacking faults well reproduce the diffuse intensities distributed between the superlattice reflections. In fact, as shown in Fig. 7.4(b), except when perfect stacking (no stacking fault) is assumed, the two-phase intergrowth model always yields smaller $R_{P}$ than the solid solution model $\left(R_{P}=0.306\right)$. The smallest value of $R_{P}=0.244$ with homogeneous stacking fault distribution is achieved at probability $40 \%$. With heterogeneous distributions of stacking faults shown in Fig. 7.4(b), the further reduced 
value of $R_{P}=0.243$ is achieved with the two-phase intergrowth model. It is observed that $\sim 75 \%$ volume fraction of $\mathrm{Li}_{2} \mathrm{MnO}_{3}$ phase exhibits $40 \%$ stacking faults, while $\sim 16 \%$ volume fraction exhibits $50 \%$ stacking faults, and $\sim 6 \%$ volume fraction exhibits $10 \%$ stacking faults. It is interesting to investigate the changes of stacking fault distributions as $\mathrm{LiCoO}_{2}$ phase is introduced with increasing content, as discussed in the following.

Fig. 7.5 compares the refinement results of two-phase intergrowth model with stacking faults and solid solution model for $10 \% \mathrm{LiCoO}_{2}-90 \% \mathrm{Li}_{2} \mathrm{MnO}_{3}(x=0.1)$. Again, stacking faults reproduce well the diffuse intensities distributed between the superlattice reflections, and the two-phase intergrowth model yields smaller $R_{P}$ than the solid solution model except at very low stacking fault probabilities. It is worth noting that, even the smallest value of $R_{P}$ with homogeneous stacking fault distribution is achieved at probability $30 \%$, the value of $R_{P}$ is further reduced with heterogeneous distributions of stacking faults, where $\sim 51 \%$ volume fraction of $\mathrm{Li}_{2} \mathrm{MnO}_{3}$ phase exhibits $20 \%$ stacking faults and $\sim 38 \%$ volume fraction exhibits $40 \%$ stacking faults. It shows that introduction of $10 \% \mathrm{LiCoO}_{2}$ phase reduces the overall stacking faults in $\mathrm{Li}_{2} \mathrm{MnO}_{3}$ phase; nevertheless, the stacking faults are not evenly distributed. It is likely that the two phases of $\mathrm{LiCoO}_{2}$ and $\mathrm{Li}_{2} \mathrm{MnO}_{3}$ are not homogeneously distributed (i.e., composition $x$ is spatially heterogeneous), where the regions of pure $\mathrm{Li}_{2} \mathrm{MnO}_{3}$ content exhibit $40 \%$ stacking faults, while the regions of lower $\mathrm{Li}_{2} \mathrm{MnO}_{3}$ content exhibit 20\% stacking faults (as shown in Fig. 7.8 for $\left.70 \% \mathrm{LiCoO}_{2}-30 \% \mathrm{Li}_{2} \mathrm{MnO}_{3}\right)$. 

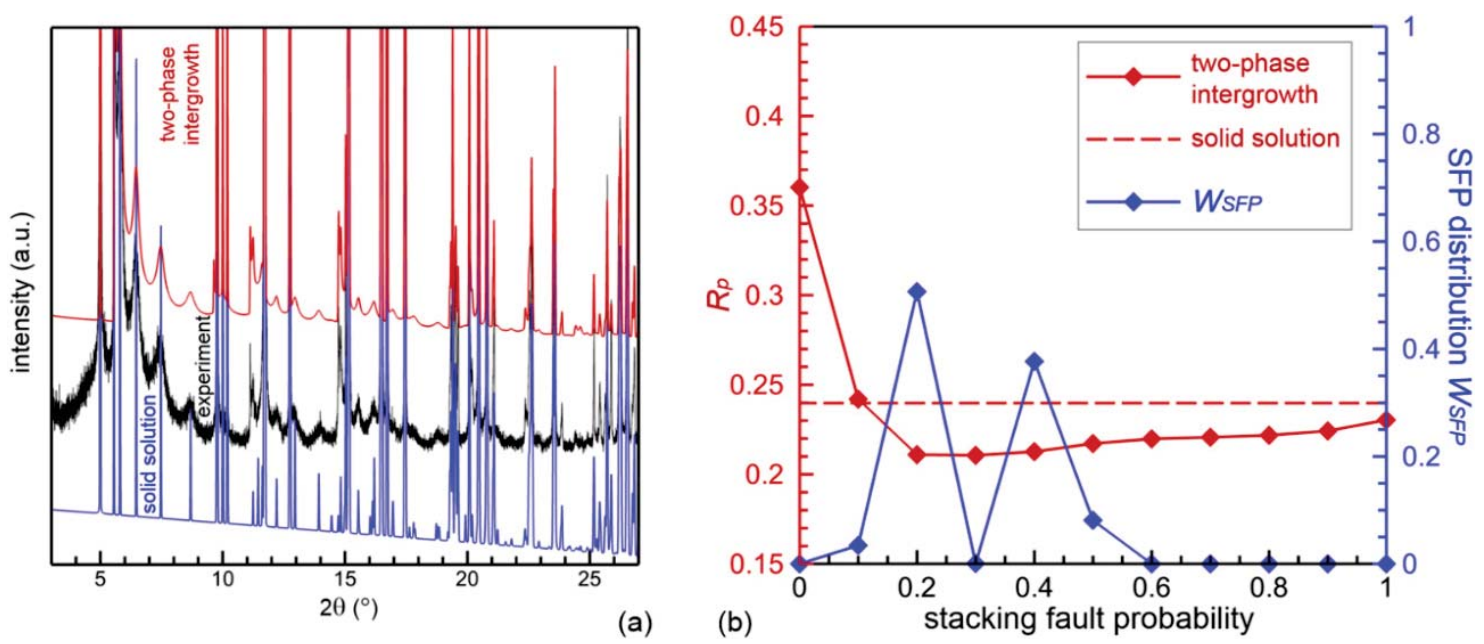

Figure 7.5 Comparison of two-phase intergrowth model with stacking faults and solid solution model for $10 \% \mathrm{LiCoO}_{2}-90 \% \mathrm{Li}_{2} \mathrm{MnO}_{3}(x=0.1)$. (a) Powder diffraction intensity profiles of experiment, solid solution model, and two-phase intergrowth model with heterogeneous distributions of stacking faults. (b) Fitting residual error $R_{P}$ of solid solution model and two-phase intergrowth model with different homogeneous stacking fault probabilities. Best fitted spatially heterogeneous distributions of stacking faults are also shown in (b).

Figure 7.6 compares the refinement results of two-phase intergrowth model with stacking faults and solid solution model for $30 \% \mathrm{LiCoO}_{2}-70 \% \mathrm{Li}_{2} \mathrm{MnO}_{3}(x=0.3)$. Again, stacking faults reproduce well the diffuse intensities distributed between the superlattice reflections, and the two-phase intergrowth model yields smaller $R_{P}$ than the solid solution model except at very low stacking fault probabilities. It is observed that $\sim 90 \%$ volume fraction of $\mathrm{Li}_{2} \mathrm{MnO}_{3}$ phase exhibits $30 \%$ stacking faults, and $\sim 9 \%$ volume fraction exhibits $20 \%$ stacking faults. The introduction of $30 \% \mathrm{LiCoO}_{2}$ phase further reduces the stacking faults in $\mathrm{Li}_{2} \mathrm{MnO}_{3}$ phase. 

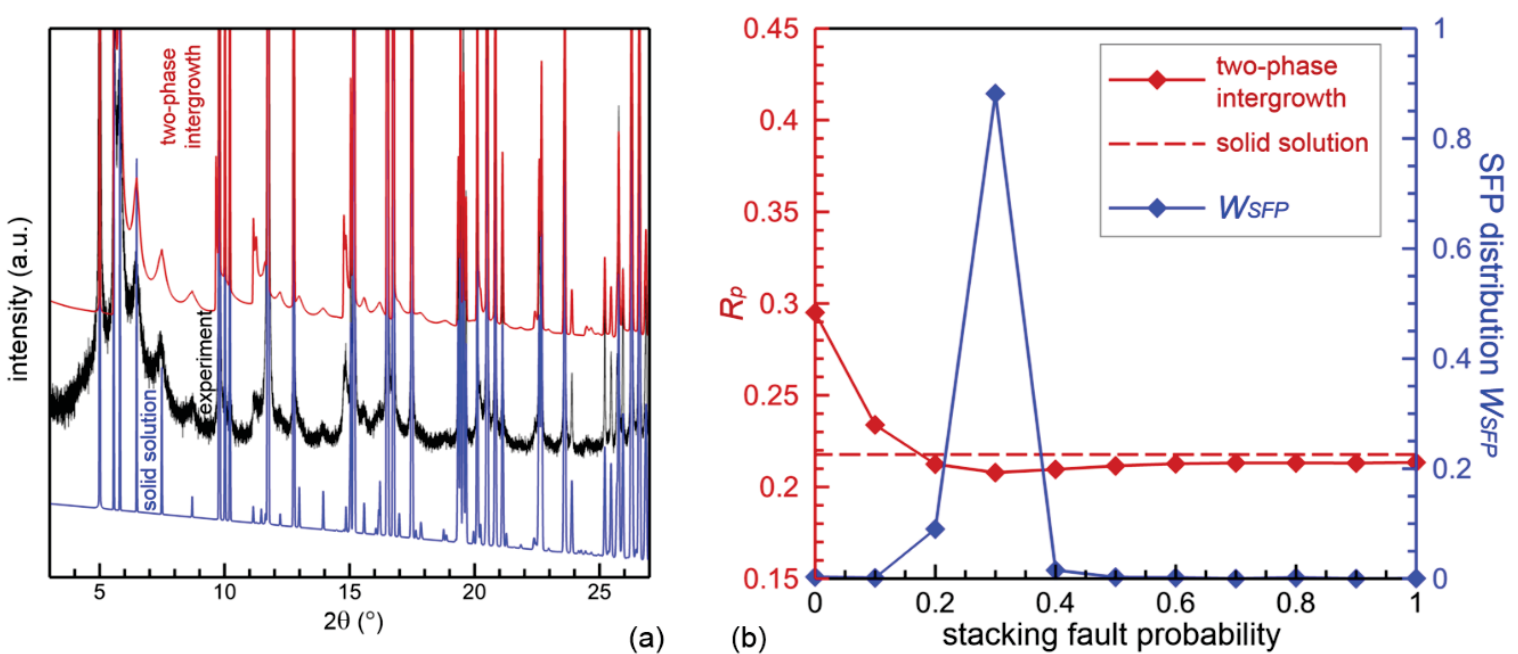

Figure 7.6 Comparison of two-phase intergrowth model with stacking faults and solid solution model for $30 \% \mathrm{LiCoO}_{2}-70 \% \mathrm{Li}_{2} \mathrm{MnO}_{3}(x=0.3)$. (a) Powder diffraction intensity profiles of experiment, solid solution model, and two-phase intergrowth model with heterogeneous distributions of stacking faults. (b) Fitting residual error $R_{P}$ of solid solution model and two-phase intergrowth model with different homogeneous stacking fault probabilities. Best fitted spatially heterogeneous distributions of stacking faults are also shown in (b).

Fig. 7.7 compares the refinement results of two-phase intergrowth model with stacking faults and solid solution model for $50 \% \mathrm{LiCoO}_{2}-50 \% \mathrm{Li}_{2} \mathrm{MnO}_{3}(x=0.5)$. Again, stacking faults reproduce well the diffuse intensities distributed between the superlattice reflections, and the two-phase intergrowth model yields smaller $R_{P}$ than the solid solution model except at low stacking fault probabilities. It is observed that $\sim 65 \%$ volume fraction of $\mathrm{Li}_{2} \mathrm{MnO}_{3}$ phase exhibits $30 \%$ stacking faults; however, it is also interesting to observe that $\sim 35 \%$ volume fraction exhibits $100 \%$ stacking faults, which will be further analyzed later with results shown in Figs. 7.11 and 7.12. 

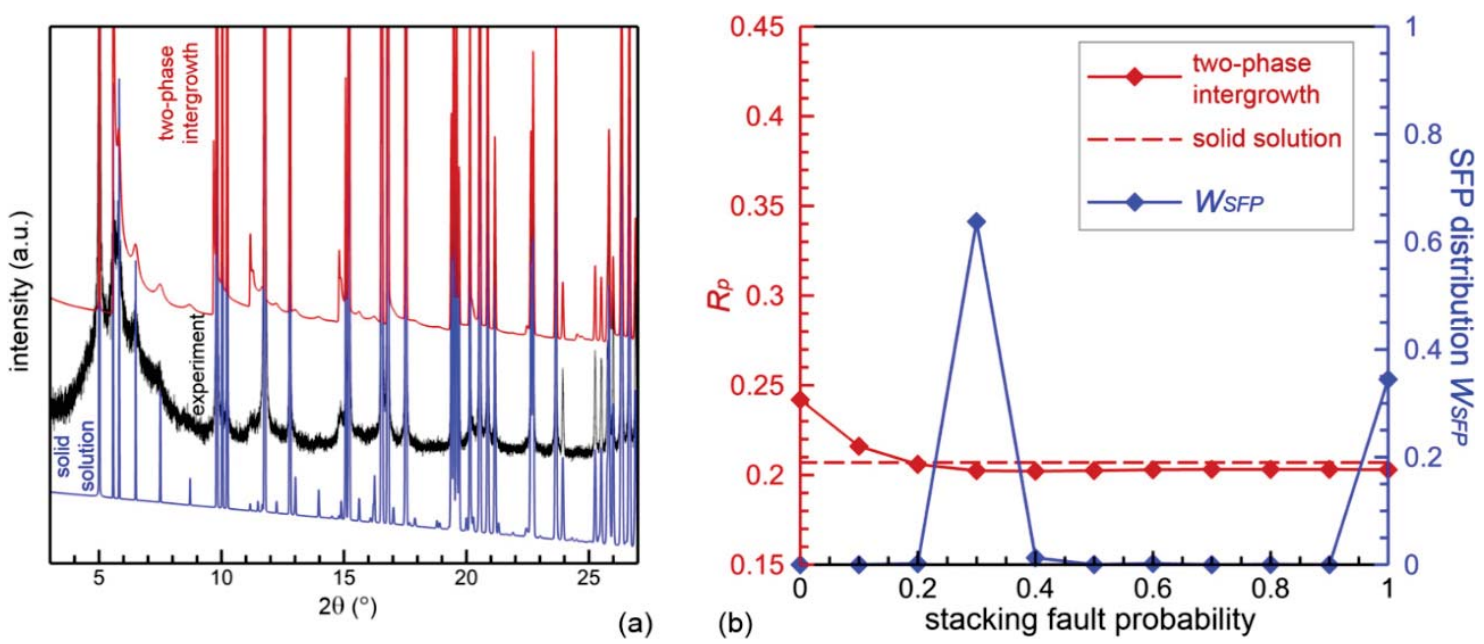

Figure 7.7 Comparison of two-phase intergrowth model with stacking faults and solid solution model for $50 \% \mathrm{LiCoO}_{2}-50 \% \mathrm{Li}_{2} \mathrm{MnO}_{3}(x=0.5)$. (a) Powder diffraction intensity profiles of experiment, solid solution model, and two-phase intergrowth model with heterogeneous distributions of stacking faults. (b) Fitting residual error $R_{P}$ of solid solution model and two-phase intergrowth model with different homogeneous stacking fault probabilities. Best fitted spatially heterogeneous distributions of stacking faults are also shown in (b).

Fig. 7.8 compares the refinement results of two-phase intergrowth model with stacking faults and solid solution model for $70 \% \mathrm{LiCoO}_{2}-30 \% \mathrm{Li}_{2} \mathrm{MnO}_{3}(x=0.7)$. Again, the two-phase intergrowth model with stacking faults gives better fitting to the experimental data than the solid solution model. The stacking faults in $\mathrm{Li}_{2} \mathrm{MnO}_{3}$ phase are further reduced, where $\sim 97 \%$ volume fraction of $\mathrm{Li}_{2} \mathrm{MnO}_{3}$ phase exhibits $20 \%$ stacking faults. 

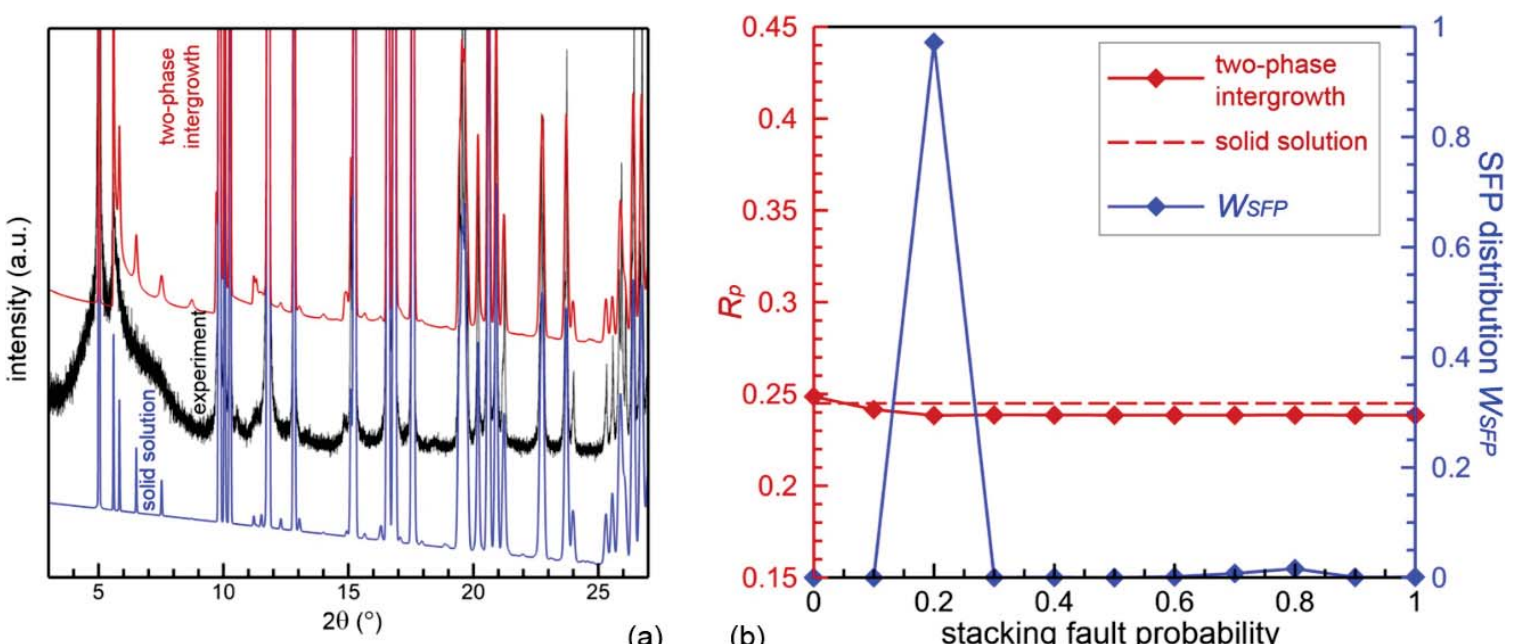

(a)

(b)

Figure 7.8 Comparison of two-phase intergrowth model with stacking faults and solid solution model for $70 \% \mathrm{LiCoO}_{2}-30 \% \mathrm{Li}_{2} \mathrm{MnO}_{3}(x=0.7)$. (a) Powder diffraction intensity profiles of experiment, solid solution model, and two-phase intergrowth model with heterogeneous distributions of stacking faults. (b) Fitting residual error $R_{P}$ of solid solution model and two-phase intergrowth model with different homogeneous stacking fault probabilities. Best fitted spatially heterogeneous distributions of stacking faults are also shown in (b).

Fig. 7.9 compares the refinement results of two-phase intergrowth model with stacking faults and solid solution model for $90 \% \mathrm{LiCoO}_{2}-10 \% \mathrm{Li}_{2} \mathrm{MnO}_{3}(x=0.9)$. With only $10 \% \mathrm{Li}_{2} \mathrm{MnO}_{3}$ phase content, while the two-phase intergrowth model with stacking faults gives better fitting to the experimental data than the solid solution model, the model is no longer sensitive to stacking fault probabilities as shown in Fig. 7.9(b), and the third-stage refinement cannot reliably find the heterogeneous stacking fault distributions any more. 

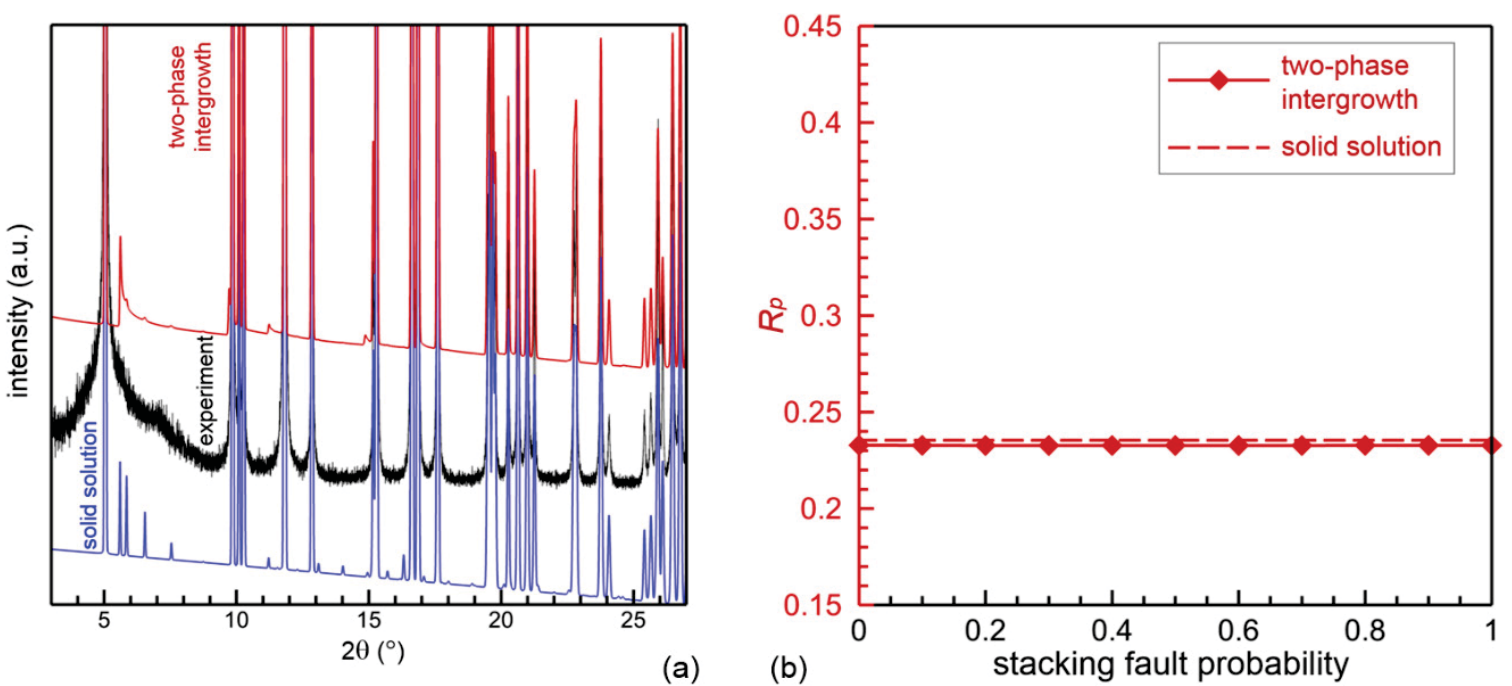

Figure 7.9 Comparison of two-phase intergrowth model with stacking faults and solid solution model for $90 \% \mathrm{LiCoO}_{2}-10 \% \mathrm{Li}_{2} \mathrm{MnO}_{3}(x=0.9)$. (a) Powder diffraction intensity profiles of experiment, solid solution model, and two-phase intergrowth model with heterogeneous distributions of stacking faults. (b) Fitting residual error $R_{P}$ of solid solution model and two-phase intergrowth model with different homogeneous stacking fault probabilities.

Fig. 7.10 shows the powder diffraction intensity profiles of experiment, solid solution model, and two-phase intergrowth model for pure $\mathrm{LiCoO}_{2}(x=1)$. The two models of $\mathrm{LiCoO}_{2}$ become identical, and there is no stacking faults involved because the pure Co layers do not suffer stacking faults. It is worth noting that DIFFaX in its current version does not support strain broadening. Therefore, in order to integrate DIFFaX and GSAS, strain broadening and other parameters that are currently not supported in DIFFaX are not considered; consideration of these additional fitting parameters would improve the fitted intensity profiles, in particular, the background intensity. 


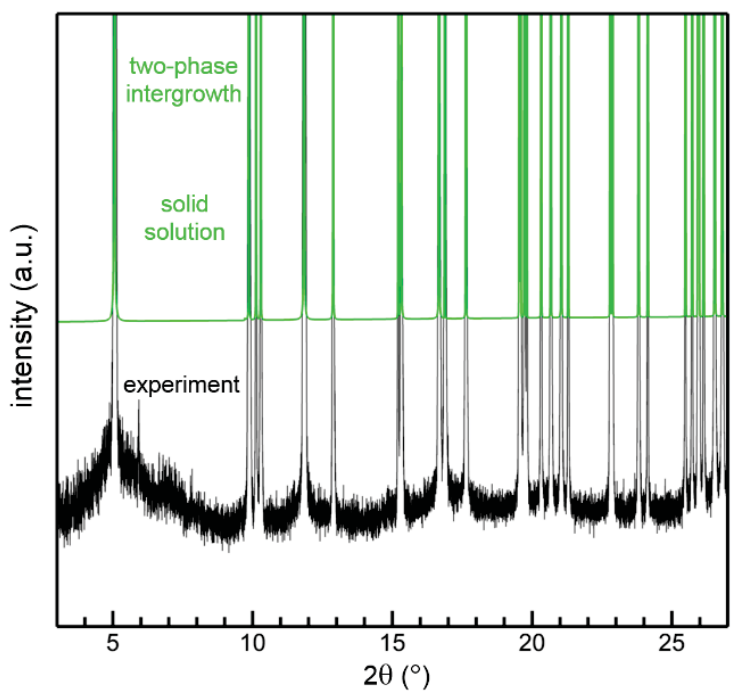

Figure 7.10 Powder diffraction intensity profiles of experiment, solid solution model, and two-phase intergrowth model for pure $\mathrm{LiCoO}_{2}(x=1)$.

As mentioned above for $50 \% \mathrm{LiCoO}_{2}-50 \% \mathrm{Li}_{2} \mathrm{MnO}_{3}(x=0.5)$ and shown in Fig. 7.7(b), in addition to $\sim 65 \%$ volume fraction of $\mathrm{Li}_{2} \mathrm{MnO}_{3}$ phase that exhibits $30 \%$ stacking faults, it is also observed that $\sim 35 \%$ volume fraction exhibits $100 \%$ stacking faults, which deserves further analysis. As described in 7.1.2(II) and illustrated in Fig. 7.2, in DIFFaX simulations stacking faults are introduced into $\mathrm{Li}_{2} \mathrm{MnO}_{3}$ phase through the probabilities of the stacking vectors, namely, V1 [0,0,1], V2 [0,1/3,1], and V3 [0,-1/3,1], where V1 yields perfect stacking while V2 and V3 produces stacking faults. In this notation, 100\% stacking faults means $0 \%$ probability of stacking vector V1, i.e., V1 is not involved in the stacking sequence. In above analysis using two-phase intergrowth model with heterogeneous distributions of stacking faults shown in Fig. 7.7, 100\% stacking faults is implemented in terms of $50 \% \mathrm{~V} 2$ and $50 \% \mathrm{~V} 3$ with random sequence among the two stacking vectors. 


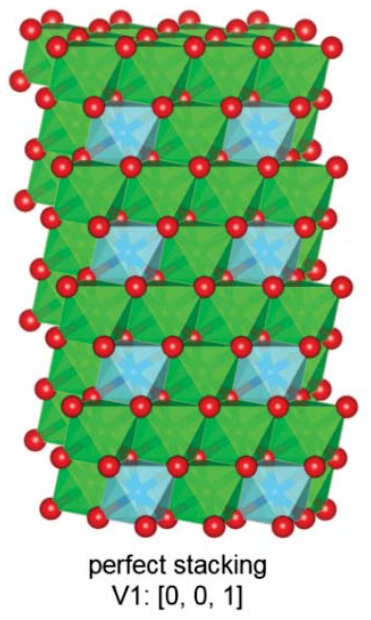

(a)

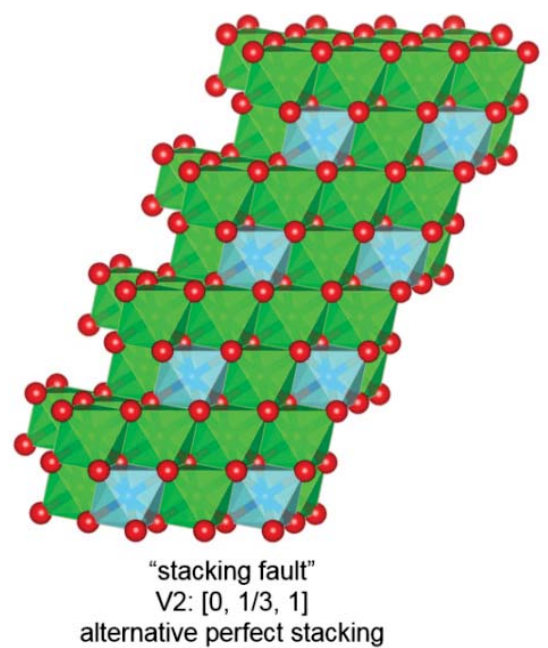

(b)

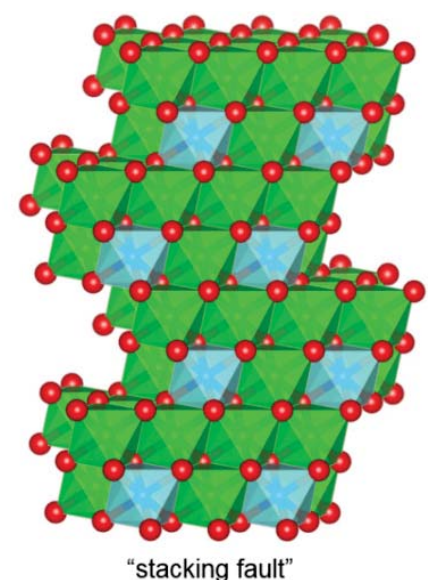

V2V3: $[0,1 / 3,1]+[0,-1 / 3,1]$ ordered stacking superlattice

(c)

Figure 7.11 Illustration of different structures of $\mathrm{Li}_{2} \mathrm{MnO}_{3}$ produced by (a) V1 stacking, (b) V2 stacking, and (c) V2V3 stacking, as visualized using VESTA.
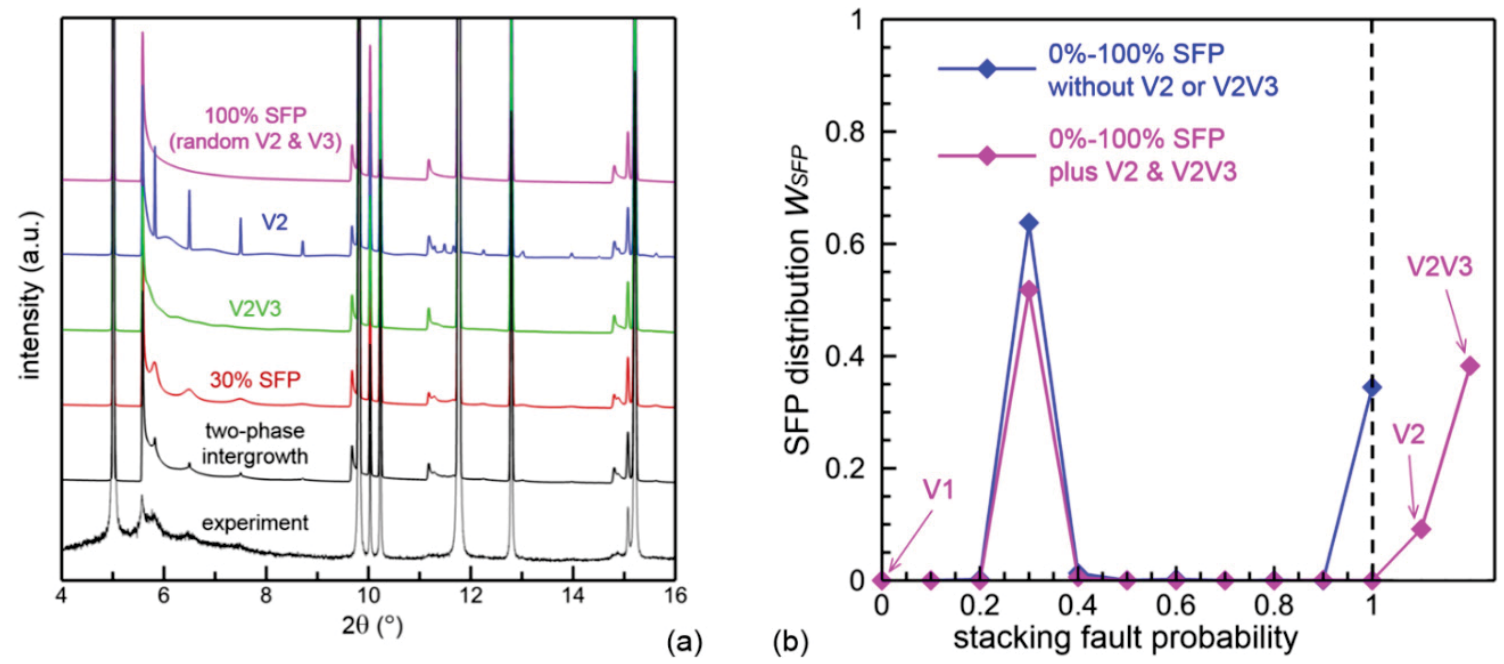

Figure 7.12 Refinement of two-phase intergrowth model with stacking faults and different structures of $\mathrm{Li}_{2} \mathrm{MnO}_{3}$ phase produced respectively by $\mathrm{V} 2$ stacking and $\mathrm{V} 2 \mathrm{~V} 3$ stacking for $50 \% \mathrm{LiCoO}_{2}-50 \% \mathrm{Li}_{2} \mathrm{MnO}_{3}(x=0.5)$. (a) Powder diffraction intensity profiles of experiment and two-phase intergrowth model with heterogeneous distributions of stacking faults. (b) Best fitted spatially heterogeneous distributions of stacking faults and stacking structures of V2 and V2V3. Blue curve is reproduced from Figure 7(b) for comparison. 
It is worth noting that there are two simple special stacking sequences that do not involve V1. In the first case, stacking involves V2 only (which is equivalent to V3 only); this case (we call it V2 stacking for convenience) indeed corresponds to an alternative perfect stacking which is different from the perfect stacking of V1 only (i.e., $0 \%$ stacking fault, we call it V1 stacking). In the second case, stacking involves both V2 and V3, and $\mathrm{V} 2$ is always followed by V3 (which is equivalent to V3 always followed by V2); this case (we call it V2V3 stacking) indeed corresponds to an ordered superlattice along the stacking direction (c-axis of the monoclinic cell). Fig. 7.11 illustrates the different structures of $\mathrm{Li}_{2} \mathrm{MnO}_{3}$ phase that are produced by $\mathrm{V} 1$ stacking, V2 stacking, and V2V3 stacking, respectively. Although there could be unlimited number of ordered stacking superlattices involving more complex V2 and V3 sequences, the two (i.e., V2 and V2V3) are the simplest and most likely ones, while more complex ones possess longer periods and tend to resemble random sequence of $50 \% \mathrm{~V} 2$ and $50 \% \mathrm{~V} 3$ (i.e., $100 \%$ stacking faults considered in Fig. 7.7). To clarify the structural nature of $100 \%$ stacking faults, we employ DIFFaX to construct the two powder diffraction intensity profiles $I_{\eta}(2 \theta)$ for $50 \% \mathrm{LiCoO}_{2}$ $50 \% \mathrm{Li}_{2} \mathrm{MnO}_{3}(x=0.5)$ where $\mathrm{Li}_{2} \mathrm{MnO}_{3}$ structure is produced respectively by $\mathrm{V} 2$ stacking and V2V3 stacking, add the two profiles into the original database of 11 profiles corresponding to $0 \%$ to $100 \%$ stacking faults, and performed the third-stage refinement on the extended database. Fig. 7.12 shows the new analysis results. The new analysis reveals that the original $100 \%$ stacking faults indeed corresponds to mostly V2V3 with some V2. As seen in Fig. 7.12(b), 53\% volume fraction of $\mathrm{Li}_{2} \mathrm{MnO}_{3}$ phase exhibits $30 \%$ stacking faults, $38 \%$ volume fraction corresponds to V2V3 ordered stacking superlattice, and $9 \%$ 
volume fraction corresponds to V2 alternative perfect stacking. Incorporation of V2 and V2V3 into the database further reduces the fitting residual error $R_{P}$ from 0.20203 to 0.20178 .

It is has been found that, as lithium ion battery cathode materials, $x \mathrm{LiCoO}_{2}-(1-$ $x) \mathrm{Li}_{2} \mathrm{MnO}_{3}$ exhibits the best electrochemical performance at composition $x=0.5$. Our systematic analysis shows that only $50 \% \mathrm{LiCoO}_{2}-50 \% \mathrm{Li}_{2} \mathrm{MnO}_{3}$ possesses the special V2V3 ordered stacking superlattice (38\% volume fraction) and V2 alternative perfect stacking (9\% volume fraction). This finding suggests that V2V3 and/or V2 stacking sequences of $\mathrm{Li}_{2} \mathrm{MnO}_{3}$ phase in the two-phase intergrowth structure could be the origin of the improved electrochemical performance. If further research confirms this structure-property relation, better battery cathode materials can be synthesized with the special structures of V2V3 ordered stacking superlattice and/or V2 alternative perfect stacking.

\subsubsection{Conclusion}

In this section, a hierarchical structural refinement approach is developed by seamlessly integrating Rietveld refinement, DIFFaX simulation and structural heterogeneity (stacking fault) refinement into a three-stage sequential fitting procedure.

The method has a capability to determine the spatially heterogeneous distributions of stacking faults in layered materials. It is employed to analyze the powder diffraction data of layered $x \mathrm{LiCoO}_{2}-(1-x) \mathrm{Li}_{2} \mathrm{MnO}_{3}$ lithium ion battery cathode materials. A two-phase intergrowth model is found to better describe the structures of $x \mathrm{LiCoO}_{2}-(1-x) \mathrm{Li}_{2} \mathrm{MnO}_{3}$ than a solid solution model, and stacking faults are prominent features of the layered materials. 
It is also found that introduction of $\mathrm{LiCoO}_{2}$ phase reduces the stacking faults in the $\mathrm{Li}_{2} \mathrm{MnO}_{3}$ phase. For $0.5 \mathrm{LiCoO}_{2}-0.5 \mathrm{Li}_{2} \mathrm{MnO}_{3}(x=0.5)$, the analysis reveals special $\mathrm{Li}_{2} \mathrm{MnO}_{3}$ structures of ordered stacking superlattice and alternative perfect stacking that coexist with the conventional $\mathrm{Li}_{2} \mathrm{MnO}_{3}$ phase with stacking faults, which suggests a structural origin of the improved electrochemical performance at this composition. Finally, the developed hierarchical structural refinement strategy provides a general approach that can further incorporate more structural phases involved in electrochemical processes (such as spinellike phases) to study structural changes during charge-discharge cycles as related to lithium extraction/insertion and capacity fade phenomena of the battery materials, where changes in phases and their volume fractions can be determined by this method.

\subsection{Analysis of Toda HE5050 Lithium Ion Battery Cathode Materials: Solid Solution, Two-Phase Intergrowth and Ordering}

Pristine Toda HE5050 is developed based on a composite concept of $0.5 \mathrm{Li}_{2} \mathrm{MnO}_{3}-$ $0.5 \mathrm{Li}\left(\mathrm{Ni}_{3 / 8} \mathrm{Co}_{1 / 4} \mathrm{Mn}_{3 / 8}\right) \mathrm{O}_{2}$, with a nominal composition $\mathrm{Li}_{1.2} \mathrm{Ni}_{0.15} \mathrm{Co}_{0.1} \mathrm{Mn}_{0.55} \mathrm{O}_{2}$. In the conventional Rietveld analysis of the high-resolution synchrotron X-ray powder diffraction data measured at the beamline 11-BM of Advanced Photon Source at Argonne National Laboratory, the following unit cell of monoclinic phase with $\mathrm{C} 2 / \mathrm{m}$ symmetry is defined. Considering the two-phase composite $0.5 \mathrm{Li}_{2} \mathrm{MnO}_{3}-0.5 \mathrm{Li}\left(\mathrm{Ni}_{3 / 8} \mathrm{Co}_{1 / 4} \mathrm{Mn}_{3 / 8}\right) \mathrm{O}_{2}$, which can be rewritten in a layered structure notation as $0.6 \mathrm{Li}\left(\mathrm{Li}_{1 / 3} \mathrm{Mn}_{2 / 3}\right) \mathrm{O}_{2}-0.4 \mathrm{Li}\left(\mathrm{Ni}_{3 / 8} \mathrm{Co}_{1 / 4} \mathrm{Mn}_{3 / 8}\right) \mathrm{O}_{2}$. For $\mathrm{Li}_{2} \mathrm{MnO}_{3}$, the ions occupy the following Wyckoff positions: $\mathrm{Li}^{2 \mathrm{c}} \mathrm{Li}^{4 \mathrm{~h}}\left(\mathrm{Li}^{2 \mathrm{~b}} \mathrm{Mn}^{4 \mathrm{~g}}\right) \mathrm{O}^{4 \mathrm{i}} \mathrm{O}^{8 \mathrm{j}}$. For $\mathrm{Li}\left(\mathrm{Ni}_{3 / 8} \mathrm{Co}_{1 / 4} \mathrm{Mn}_{3 / 8}\right) \mathrm{O}_{2}, 2 \mathrm{~b}$ sites are assumed to be occupied by Ni due to its similar size 
to $\mathrm{Li}$, and the ions occupy the following Wyckoff positions: $\mathrm{Li}^{2 \mathrm{c}} \mathrm{Li}^{4 \mathrm{~h}}\left[\mathrm{Ni}^{2 \mathrm{~b}}\left(\mathrm{Ni}_{1 / 16} \mathrm{Co}_{3 / 8} \mathrm{Mn}_{9 / 16}\right)^{4 \mathrm{~g}}\right] \mathrm{O}^{4 \mathrm{i}} \mathrm{O}^{8 \mathrm{j}}$. To construct the unit cell for Rietveld refinement, a partially ordered solid solution (will be called disordered solid solution hereafter for convenience, in contrast to ordered solid solution without the following ion exchange) is assumed, where the following ion exchanges $x, y$ and $z$ are allowed: exchange $x$ between inter-layer $\mathrm{Li}^{2 \mathrm{c}}$ and $\mathrm{Ni}^{2 \mathrm{~b}}$, exchange $y$ between inter-layer $\mathrm{Li}^{4 \mathrm{~h}}$ and $\mathrm{Ni}^{4 \mathrm{~g}}$, and exchange $z$ between intra-layer $\mathrm{Li}^{2 \mathrm{~b}}$ and $\mathrm{Mn}^{4 \mathrm{~g}}$. The monoclinic unit cell can be written as

$$
\left(\mathrm{Li}_{1-x} \mathrm{Ni}_{x}\right)^{2 \mathrm{c}}\left(\mathrm{Li}_{1-y} \mathrm{Ni}_{y}\right)^{4 \mathrm{~h}}\left[\left(\mathrm{Li}_{3 / 5+x-2 z} \mathrm{Ni}_{2 / 5-x} \mathrm{Mn}_{2 z}\right)^{2 \mathrm{~b}}\left(\mathrm{Li}_{y+z} \mathrm{Ni}_{1 / 40-y} \mathrm{Co}_{3 / 20} \mathrm{Mn}_{33 / 40-z}\right)^{4 \mathrm{~g}}\right] \mathrm{O}^{4 \mathrm{i}} \mathrm{O}^{8 \mathrm{j}},
$$

Rietveld refinement is performed using GSAS [216], which gives the following best fitted results: monoclinic lattice parameters, $a_{\mathrm{m}}=4.94610(8) \AA, b_{\mathrm{m}}=8.56459(9) \AA, c_{\mathrm{m}}=5.02830(6)$ $\AA, \beta=109.178(3)^{\circ}$; fractional coordinates and mean square atomic vibration amplitude for Wyckoff positions, $(0,0,1 / 2)$ and $U_{\text {iso }}=0.00100$ for 2 c sites, $(0,0.320318,1 / 2)$ and $U_{\text {iso }}=0.01641$ for $4 \mathrm{~h}$ sites, $(0,1 / 2,0)$ and $U_{\text {iso }}=0.00315$ for $2 \mathrm{~b}$ sites, $(0,0.167555,0)$ and $U_{\text {iso }}=0.00185$ for $4 \mathrm{~g}$ sites, $(0.223412,0,0.223093)$ and $U_{\text {iso }}=0.00148$ for $4 \mathrm{i}$ sites, $(0.255421$, $0.321697,0.226578)$ and $U_{\text {iso }}=0.00389$ for $8 \mathrm{j}$ sites; ion exchanges, $x=0.0001, y=0.01$, $z=0.1176 ; \quad$ pseudo-Voigt broadening parameters, $u=0.0116300, \quad v=-0.00126000$, $w=0.00681449, \eta=0.487082$.

When modeled as a disordered solid solution described by Eq. 7.4, the conventional Rietveld analysis describes pristine Toda HE5050 as

$$
\begin{aligned}
& \left(\mathrm{Li}_{0.9999} \mathrm{Ni}_{0.0001}\right)^{2 \mathrm{c}}\left(\mathrm{Li}_{0.99} \mathrm{Ni}_{0.01}\right)^{4 \mathrm{~h}}\left[\left(\mathrm{Li}_{0.3649} \mathrm{Ni}_{0.3999} \mathrm{Mn}_{0.2352}\right)^{2 \mathrm{~b}},\right. \\
& \left.\left(\mathrm{Li}_{0.1276} \mathrm{Ni}_{0.015} \mathrm{Co}_{0.15} \mathrm{Mn}_{0.7074}\right)^{4 \mathrm{~g}}\right] \mathrm{O}^{4 \mathrm{i}} \mathrm{O}^{8 \mathrm{j}}
\end{aligned}
$$


It is worth noting the following observations. (i) The exchange between inter-layer $\mathrm{Li}^{2 \mathrm{c}}$ and $\mathrm{Ni}^{2 \mathrm{~b}}$ is practically absent $(x=0.0001)$, and the exchange between inter-layer $\mathrm{Li}^{4 \mathrm{~h}}$ and $\mathrm{Ni}^{4 \mathrm{~g}}$ is very small $(y=0.01)$, thus the Li layer contains essentially Li ions only. (ii) The exchange between intra-layer $\mathrm{Li}^{2 \mathrm{~b}}$ and $\mathrm{Mn}^{4 \mathrm{~g}}$ is non-negligible $(z=0.1176)$, leading to a disordered (partially ordered) structure. (iii) The lattice parameters $a_{\mathrm{m}}$ and $b_{\mathrm{m}}$ exhibit a ratio $b_{\mathrm{m}} / a_{\mathrm{m}}=1.7316$ which is very close to $\sqrt{3}$. (iv) The conventional Rietveld method does not fit the experimental pattern very well, due to the presence of stacking faults associated with the ordered transition metal layers in which Li and Ni preferably occupy $2 \mathrm{~b}$ sites.

It is worth noting that the effects of stacking faults on diffraction are not explicitly considered in the conventional Rietveld analysis; instead, their average effects are manifested in the fitted structural parameters of the unit cell. In particular, higher ion exchanges (thus higher degree of disorder) are obtained as reflected in the fitted site occupancies, i.e., $\mathrm{Li}^{2 \mathrm{~b}}$ and $\mathrm{Mn}^{4 \mathrm{~g}}$ exchange. To explicitly take into account the stacking faults and study their effects on diffraction, DIFFaX software is employed to perform computational analysis to gain quantitative insights into the structural characteristics. For comparison, an ordered solid solution (without ion exchange, i.e., $x=y=z=0$ ) is also considered, whose unit cell is described by

$$
\mathrm{Li}^{2 \mathrm{c}} \mathrm{Li}^{4 \mathrm{~h}}\left[\left(\mathrm{Li}_{3 / 5} \mathrm{Ni}_{2 / 5}\right)^{2 \mathrm{~b}}\left(\mathrm{Ni}_{1 / 40} \mathrm{Co}_{3 / 20} \mathrm{Mn}_{33 / 40}\right)^{4 \mathrm{~g}}\right] \mathrm{O}^{4 \mathrm{i}} \mathrm{O}^{8 \mathrm{j}}
$$

In order to shed light on the structural nature of solid solution versus two-phase intergrowth, a two-phase composite model is constructed as follows

$$
0.6 \mathrm{Li}^{2 \mathrm{c}} \mathrm{Li}^{4 \mathrm{~h}}\left(\mathrm{Li}^{2 \mathrm{~b}} \mathrm{Mn}^{4 \mathrm{~g}}\right) \mathrm{O}^{4 \mathrm{i}} \mathrm{O}^{8 \mathrm{j}}-0.4 \mathrm{Li}^{2 \mathrm{c}} \mathrm{Li}^{4 \mathrm{~h}}\left[\mathrm{Ni}^{2 \mathrm{~b}}\left(\mathrm{Ni}_{1 / 16} \mathrm{Co}_{3 / 8} \mathrm{Mn}_{9 / 16}\right)^{4 \mathrm{~g}}\right] \mathrm{O}^{4 \mathrm{i}} \mathrm{O}^{8 \mathrm{j}}
$$


where $\mathrm{Li}_{2} \mathrm{MnO}_{3}$ and $\mathrm{Li}\left(\mathrm{Ni}_{3 / 8} \mathrm{Co}_{1 / 4} \mathrm{Mn}_{3 / 8}\right) \mathrm{O}_{2}$ are assumed to be perfectly ordered with their 2b sites occupied exclusively by $\mathrm{Li}$ and $\mathrm{Ni}$ ions, respectively.

\subsubsection{Structural Nature of Solid Solution versus Two-Phase Intergrowth}

DIFFaX analysis is performed based on above Rietveld analysis results. Specifically, the same monoclinic lattice parameters and Wyckoff position fractional coordinates as fitted by Rietveld refinement are used in all three models described in Eqs. (7.5)-(7.7), namely, disorder solid solution, ordered solid solution, and two-phase intergrowth. The mean square atomic vibration amplitudes for individual positions are assumed to be the same as fitted for disordered solid solution and are used for ordered solid solution and two-phase intergrowth, irrespective of the atom occupancies in different structural models. The atom occupancy on each site is defined by Eqs. (7.5)-(7.7). The same fitted pseudo-Voigt broadening parameters are used in all cases. It is worth noting that such occupancy-independent structure assumptions are necessary simplifications in order to perform DIFFaX analysis, since the occupancy-dependent structural parameters cannot be obtained from the conventional Rietveld refinement for the intergrowth phases of $\mathrm{Li}_{2} \mathrm{MnO}_{3}$ and $\mathrm{Li}\left(\mathrm{Ni}_{3 / 8} \mathrm{Co}_{1 / 4} \mathrm{Mn}_{3 / 8}\right) \mathrm{O}_{2}$, where coherent scattering and interference effects must be taken into account (unlike the coexistence of macro-domained phases that can be analyzed by Rietveld refinement in terms of phase volume fractions). The stacking sequence is described by three stacking translation vectors, where $[0,0,1]$ corresponds to perfect stacking without fault, and $[0,1 / 3,1]$ and $[0,-1 / 3,1]$ correspond to two types of stacking faults associated with the ordered transition metal layers (while oxygen layers do not suffer stacking faults). Fig. 7.13 illustrates the stacking sequence, stacking fault, and 
two-phase intergrowth as visualized by VESTA [199] and implemented in DIFFaX simulations, where both phase molar fractions and stacking fault probabilities can be specified by control parameters. The DIFFaX simulation results are presented in the following, and are discussed to identify the nature of solid solution versus two-phase intergrowth and to estimate composition and stacking fault percentage as related to pristine Toda HE5050.

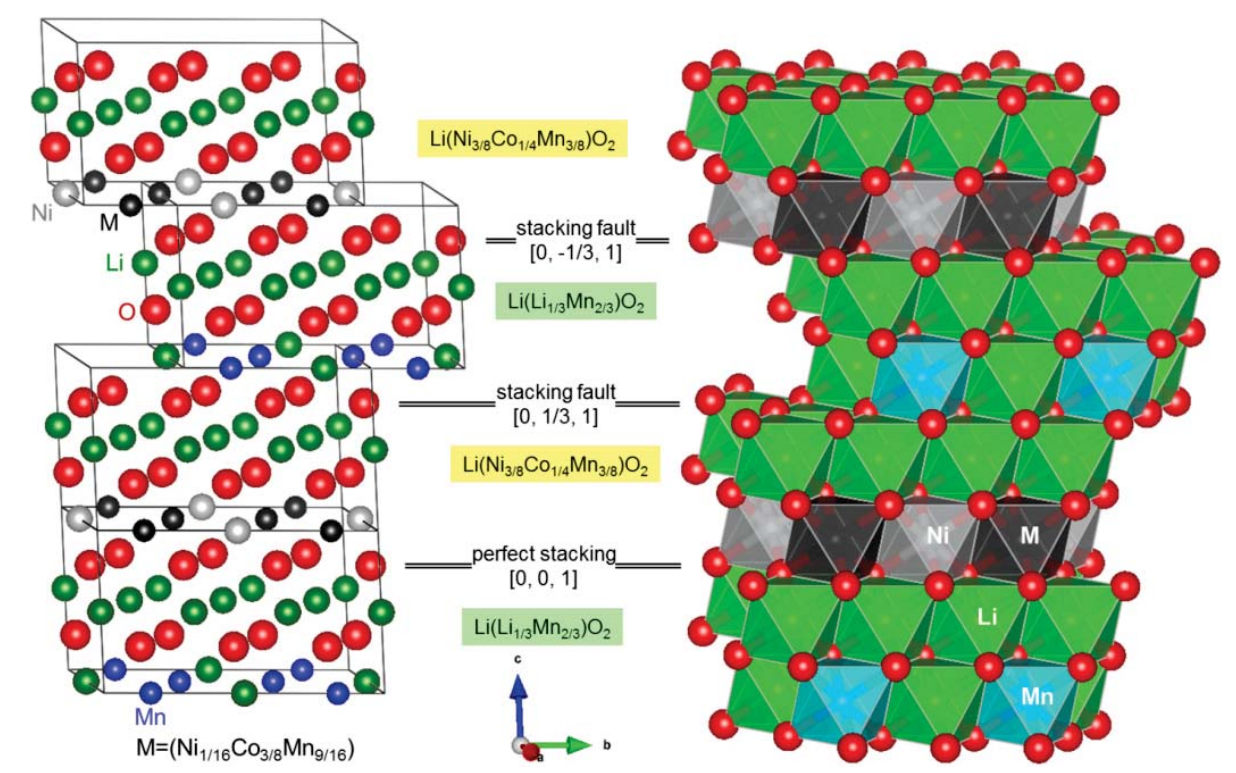

Figure 7.13 DIFFaX implementation of unit cells, stacking sequence, stacking fault, and two-phase intergrowth, as visualized using VESTA. 

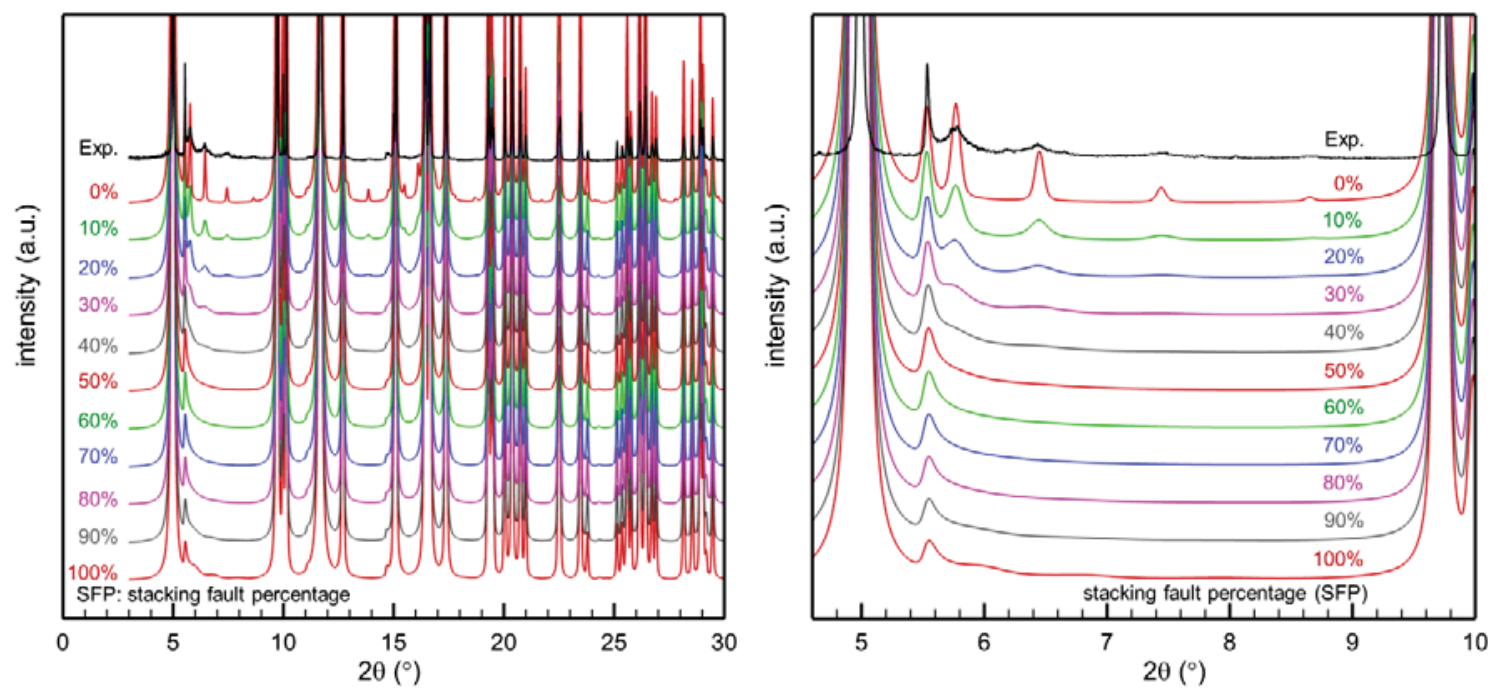

Figure 7.14 DIFFaX simulation of X-ray powder diffraction of disordered solid solution $\mathrm{Li}_{1.2} \mathrm{Ni}_{0.15} \mathrm{Co}_{0.1} \mathrm{Mn}_{0.55} \mathrm{O}_{2}$ with different percentages of stacking faults. Right panel is a detailed view of the left panel in $2 \theta$ range between $4.6^{\circ}-10^{\circ}$. Experimental profile is plotted for comparison.
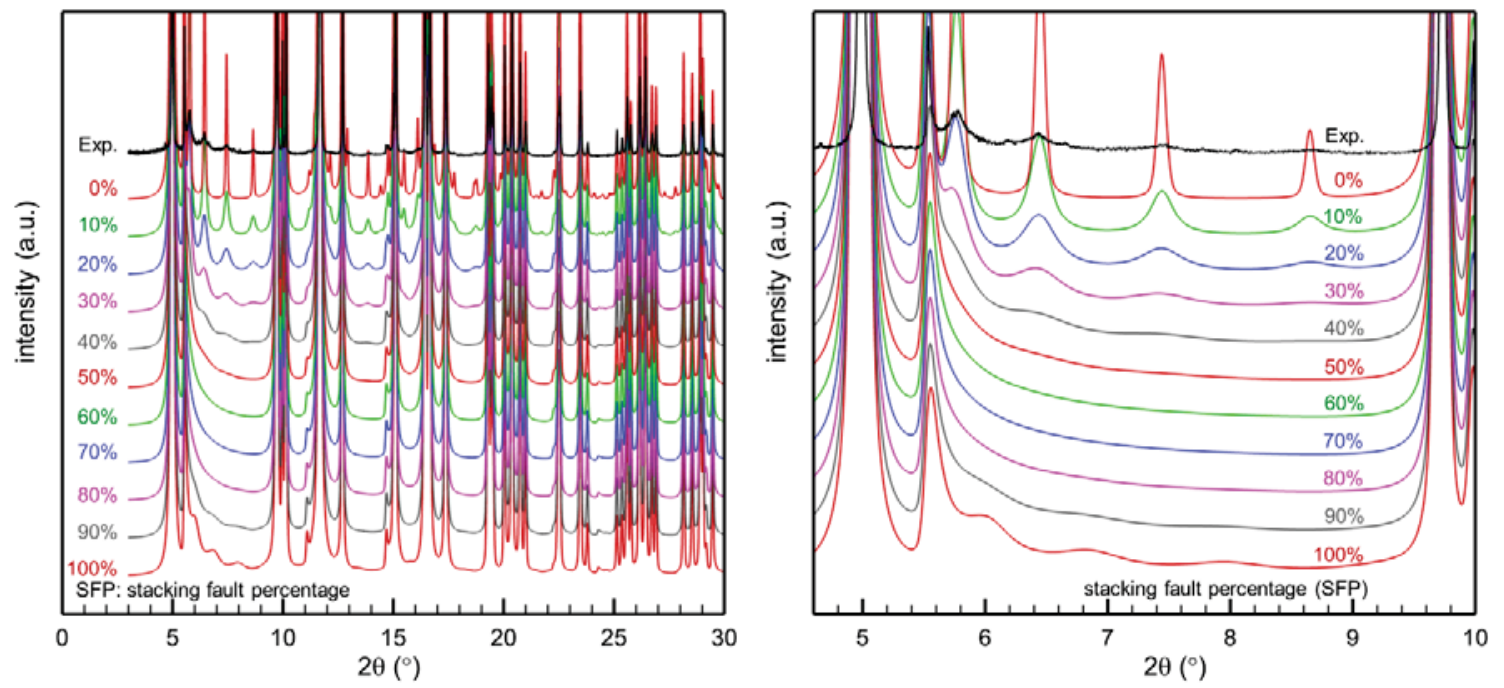

Figure 7.15 DIFFaX simulation of X-ray powder diffraction of ordered solid solution $\mathrm{Li}_{1.2} \mathrm{Ni}_{0.15} \mathrm{Co}_{0.1} \mathrm{Mn}_{0.55} \mathrm{O}_{2}$ with different percentages of stacking faults. Right panel is a detailed view of the left panel in $2 \theta$ range between $4.6^{\circ}-10^{\circ}$. Experimental profile is plotted for comparison. 
The DIFFaX simulated X-ray power diffraction profiles are shown in Figs. 7.14, 7.15 and 7.16 respectively for disordered solid solution, ordered solid solution, and twophase intergrowth with different percentages of stacking faults. As a general observation in all three models, the ordered (partially ordered) transition metal layers produce superlattice reflections, which are gradually smoothed out by increasing percentages of stacking faults. While their diffraction profiles are all qualitatively similar to the experimental profile, these three structure models can be distinguished by quantitative analysis of their diffraction profiles.
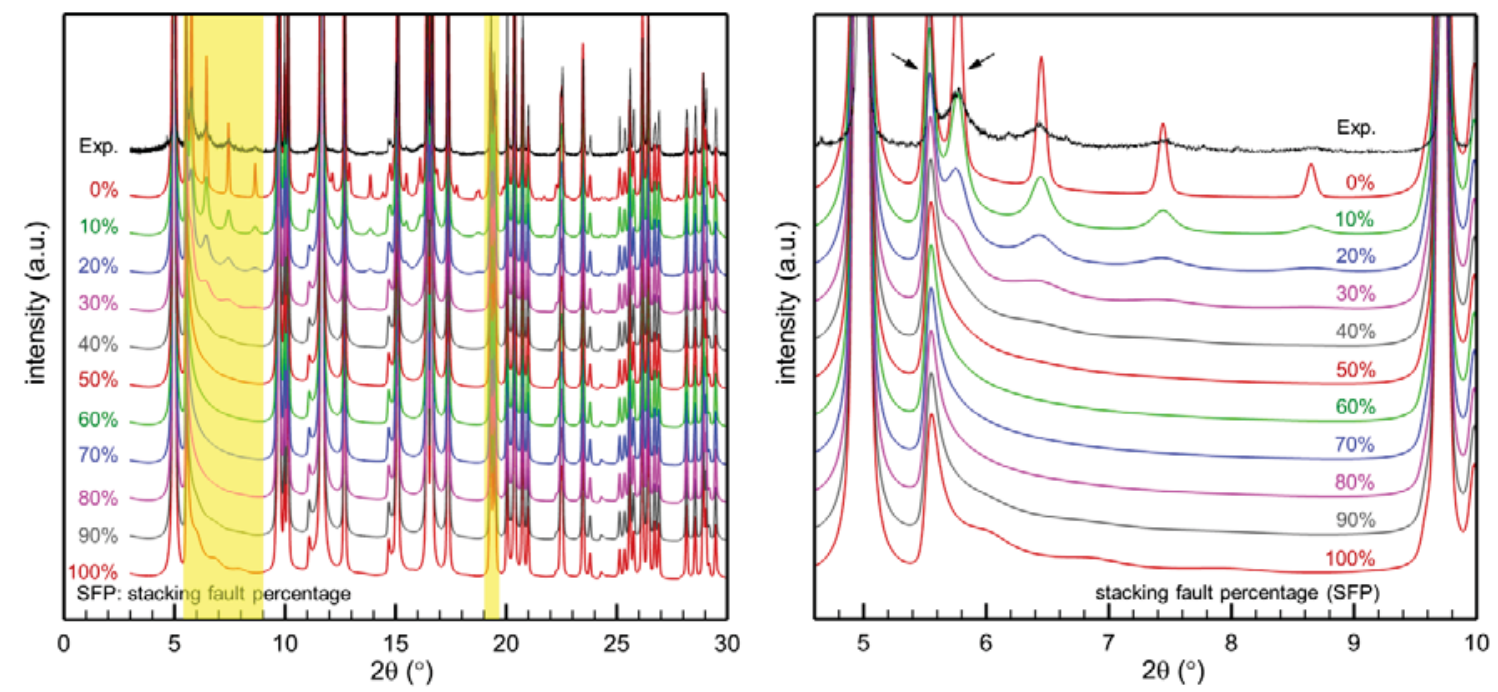

Figure 7.16 DIFFaX simulation of X-ray powder diffraction of two-phase intergrowth of $0.5 \mathrm{Li}_{2} \mathrm{MnO}_{3}-0.5 \mathrm{Li}\left(\mathrm{Ni}_{3 / 8} \mathrm{Co}_{1 / 4} \mathrm{Mn}_{3 / 8}\right) \mathrm{O}_{2}$ with different percentages of stacking faults. Right panel is a detailed view of the left panel in $2 \theta$ range between $4.6^{\circ}-10^{\circ}$. Experimental profile is plotted for comparison. The $2 \theta$ ranges highlighted in yellow and superlattice reflection peaks highlighted by arrows are used for quantitative analysis of composition and stacking fault percentage. 
To quantitatively distinguish the three structure models described in Eqs. (7.5)(7.7), we explore the stacking fault-sensitive features of the diffraction profiles. As highlighted in Fig. 7.16 (yellow shaded in left panel), the superlattice reflections in the $2 \theta$ range of $5.3^{\circ}-9^{\circ}$ sensitively depend on the percentage of stacking faults, while the peak in the $2 \theta$ range of $19^{\circ}-19.7^{\circ}$ is relatively insensitive to stacking faults. Thus, the ratio of the integrated intensities over these two $2 \theta$ ranges can serve as a criterion to distinguish the three structure models. Such integrated intensity ratios are plotted in Fig. 7.17 for the three structure models with $0-100 \%$ stacking faults and compared with experimental data. It shows that two-phase intergrowth model agrees with the experiment, while solid solution models (either ordered or disordered) do not agree with experiment. Therefore, the DIFFaX simulations support the two-phase intergrowth model of pristine Toda HE5050. It is worth noting that the ordered solid solution model is closer to the experimental data than the disordered solid solution model, which shows that the conventional Rietveld analysis overestimates the ion exchanges (degree of disorder) due to the average effect of stacking faults. 


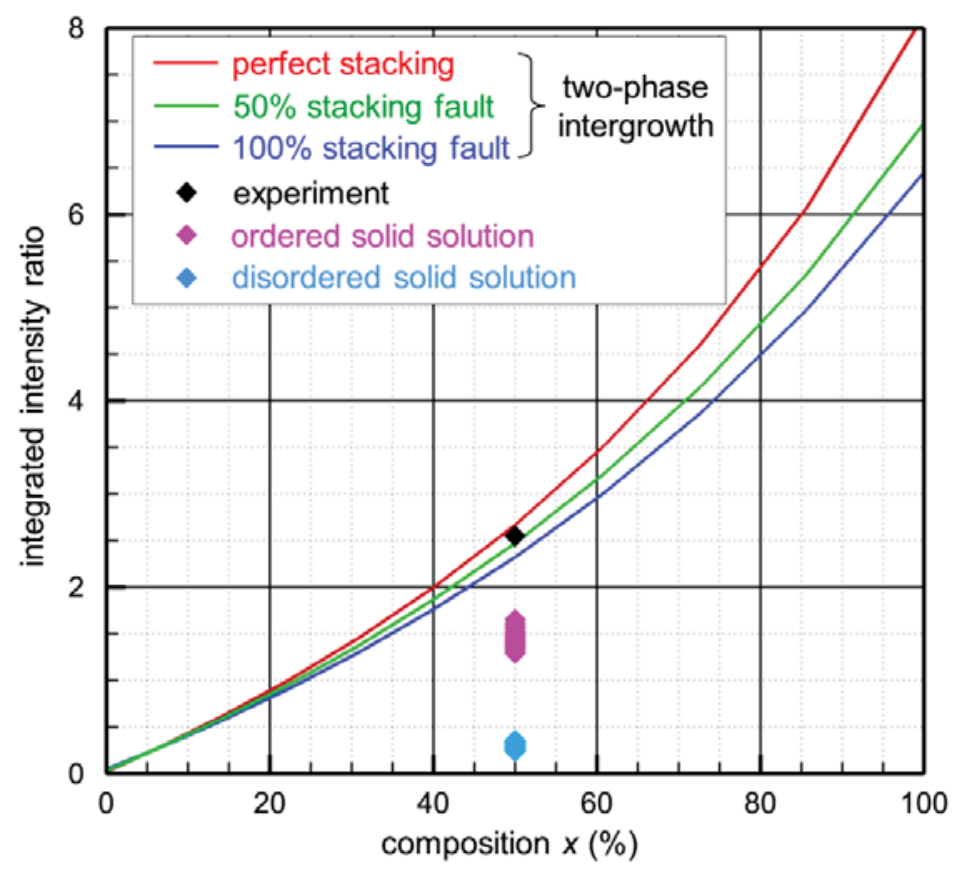

Figure 7.17 Distinction of different structure models (disordered solid solution, ordered solid solution, two-phase intergrowth) and determination of composition $x$ of $x \mathrm{Li}_{2} \mathrm{MnO}_{3}$ $(1-x) \mathrm{Li}\left(\mathrm{Ni}_{3 / 8} \mathrm{Co}_{1 / 4} \mathrm{Mn}_{3 / 8}\right) \mathrm{O}_{2}$ via quantitative comparison of $\mathrm{DIFFaX}$ simulations with experimental data. Two-phase intergrowth agrees with Toda HE5050 experiment, while solid solution (either ordered or disordered) does not agree with experiment.

Fig. 7.18 plots the simulated diffraction profiles of $x \mathrm{Li}\left(\mathrm{Li}_{1 / 3} \mathrm{Mn}_{2 / 3}\right) \mathrm{O}_{2}-(1-$ $x) \mathrm{Li}\left(\mathrm{Ni}_{3 / 8} \mathrm{Co}_{1 / 4} \mathrm{Mn}_{3 / 8}\right) \mathrm{O}_{2}$ two-phase intergrowth with $20 \%$ stacking faults and different composition $x$ to illustrate the effects of composition (two phase fractions) on diffraction. Figs. 7.17 and 7.18 shows that it is possible to estimate the composition $x$ of $x \mathrm{Li}_{2} \mathrm{MnO}_{3}-(1-$ $x) \mathrm{Li}\left(\mathrm{Ni}_{3 / 8} \mathrm{Co}_{1 / 4} \mathrm{Mn}_{3 / 8}\right) \mathrm{O}_{2}$ by quantitatively comparing the integrated intensity ratio from experimental profile with the composition-dependent ratio curve as constructed by DIFFaX simulations. In addition, it is also possible to further estimate the stacking fault percentage in similar manner. 

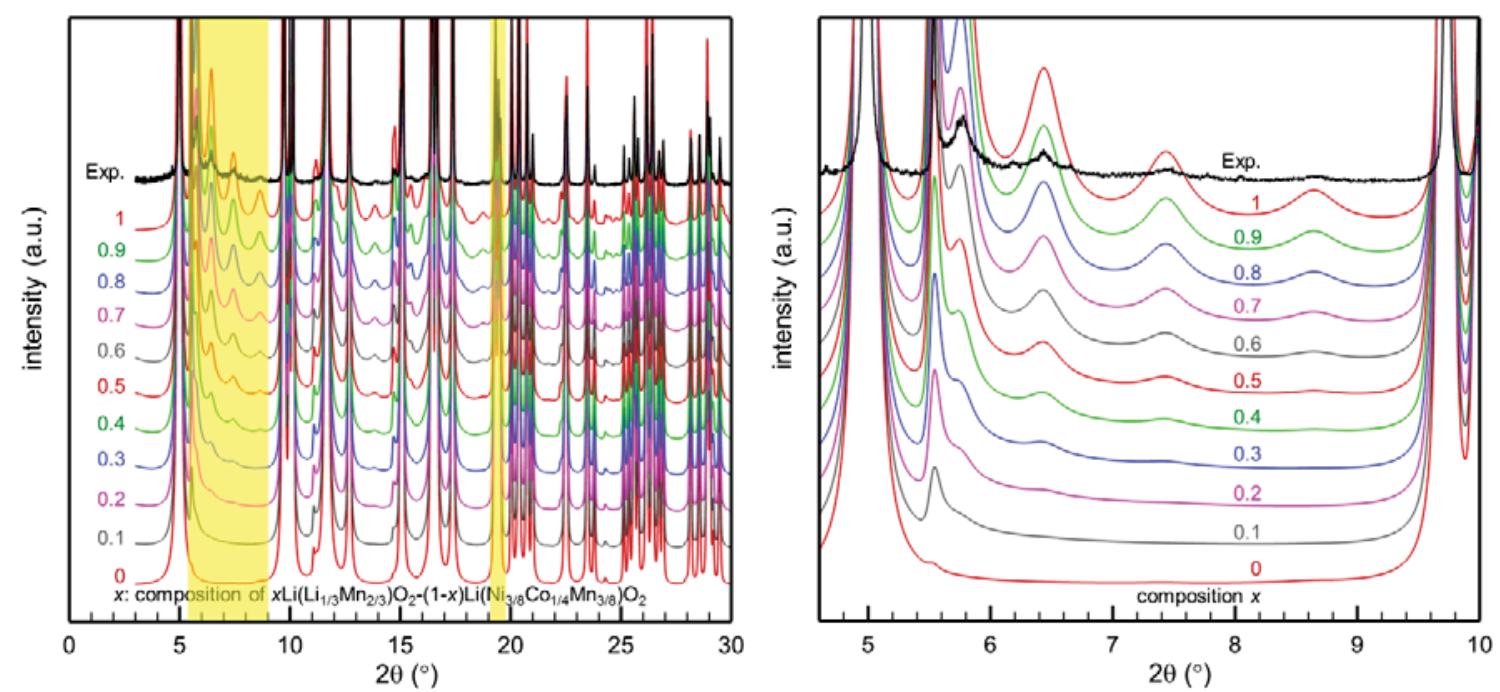

Figure 7.18 DIFFaX simulation of X-ray powder diffraction of $x \mathrm{Li}\left(\mathrm{Li}_{1 / 3} \mathrm{Mn}_{2 / 3}\right) \mathrm{O}_{2}-(1-$ $x) \mathrm{Li}\left(\mathrm{Ni}_{3 / 8} \mathrm{Co}_{1 / 4} \mathrm{Mn}_{3 / 8}\right) \mathrm{O}_{2}$ two-phase intergrowth with $20 \%$ stacking faults and different composition $x$. Right panel is a detailed view of the left panel in $2 \theta$ range between $4.6^{\circ}$ $10^{\circ}$. Experimental profile is plotted for comparison.
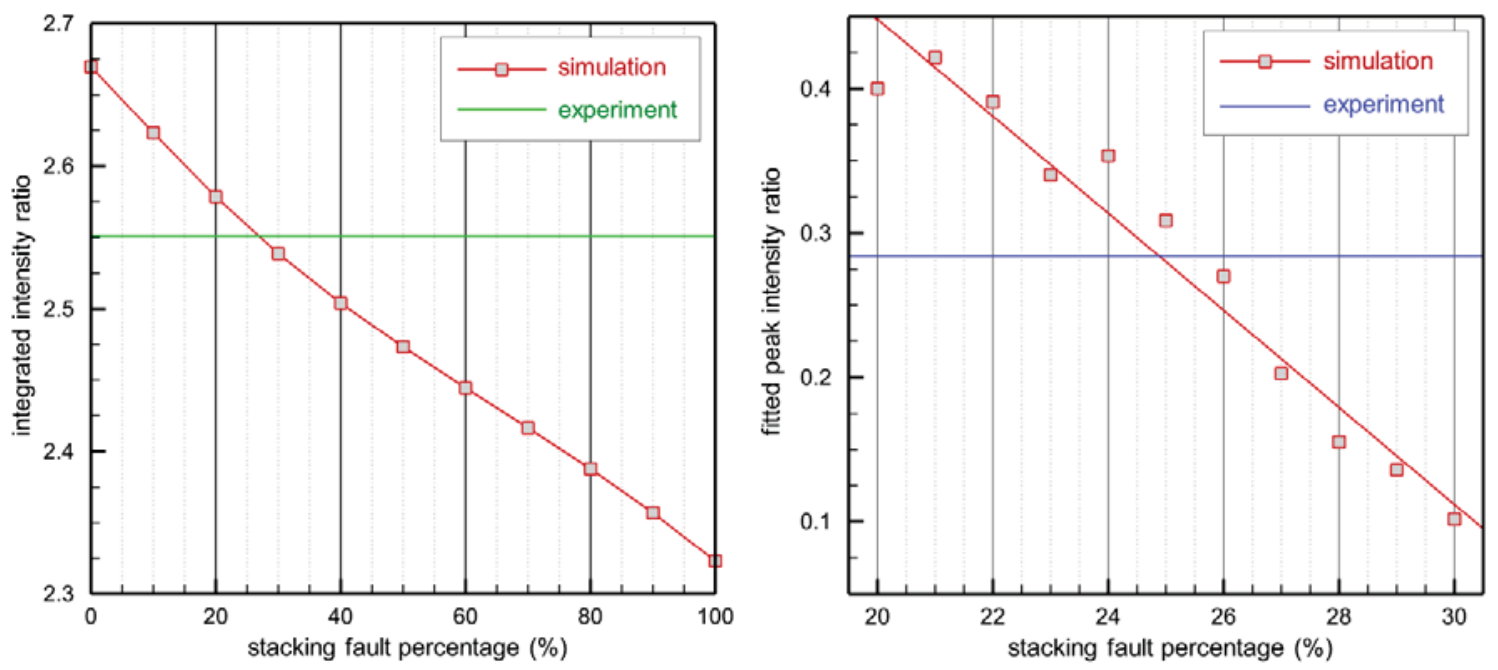

Figure 7.19 Determination of stacking fault percentage of $0.5 \mathrm{Li}_{2} \mathrm{MnO}_{3}-$ $0.5 \mathrm{Li}\left(\mathrm{Ni}_{3 / 8} \mathrm{Co}_{1 / 4} \mathrm{Mn}_{3 / 8}\right) \mathrm{O}_{2}$ (Toda $\mathrm{HE} 5050$ ) via quantitative comparison of DIFFaX simulations with experimental data based on (left panel) integrated intensity ratio over the two $2 \theta$ ranges highlighted in left panel of Fig. 7.16 or (right panel) fitted peak intensity ratio of the two superlattice reflections highlighted in right panel of Fig. 7.16. Stacking fault percentage is estimated to be $\sim 25 \%$ in both methods. 
Once the composition is determined, the diffraction profiles are simulated by DIFFaX for different percentages of stacking faults. The left panel of Fig. 7.19 plots the integrated intensity ratio of $0.5 \mathrm{Li}_{2} \mathrm{MnO}_{3}-0.5 \mathrm{Li}\left(\mathrm{Ni}_{3 / 8} \mathrm{Co}_{1 / 4} \mathrm{Mn}_{3 / 8}\right) \mathrm{O}_{2}$ (Toda HE5050 twophase intergrowth) as a function of stacking fault percentage. Comparison with experimental data estimates $\sim 25 \%$ stacking faults in pristine Toda HE5050. It is also feasible to exploit other stacking fault-sensitive features of the diffraction data, such as the superlattice reflection peaks highlighted by arrows in Fig. 7.16 (right panel). Fitting these two peaks using pseudo-Voigt functions gives their respective intensities. The right panel of Fig. 7.19 plots the fitted peak intensity ratio of $0.5 \mathrm{Li}_{2} \mathrm{MnO}_{3}-0.5 \mathrm{Li}\left(\mathrm{Ni}_{3 / 8} \mathrm{Co}_{1 / 4} \mathrm{Mn}_{3 / 8}\right) \mathrm{O}_{2}$ as a function of stacking fault percentage. Comparison with experimental data also estimates $\sim 25 \%$ stacking faults, in agreement with the previous method.

Fig. 7.20 plots the DIFFaX simulated diffraction profiles of $0.5 \mathrm{Li}_{2} \mathrm{MnO}_{3}$ $0.5 \mathrm{Li}\left(\mathrm{Ni}_{3 / 8} \mathrm{Co}_{1 / 4} \mathrm{Mn}_{3 / 8}\right) \mathrm{O}_{2}$ (Toda HE5050 two-phase intergrowth) with $20 \%$ and $25 \%$ stacking faults (as estimated from above study) and comparison with experimental profile. While the two-phase intergrowth model with stacking faults reproduces well the major features of the experimental profile, there are still some minor features, such as the weak peaks highlighted by arrows in Fig. 7.20 (right panel) that cannot be explained by the twophase intergrowth model. It implies that there could exist minor phases other than $\mathrm{Li}_{2} \mathrm{MnO}_{3}$ and $\mathrm{Li}\left(\mathrm{Ni}_{3 / 8} \mathrm{Co}_{1 / 4} \mathrm{Mn}_{3 / 8}\right) \mathrm{O}_{2}$. Such possibilities will be investigated in subsequent study together with the structural changes during charge-discharge cycles. 

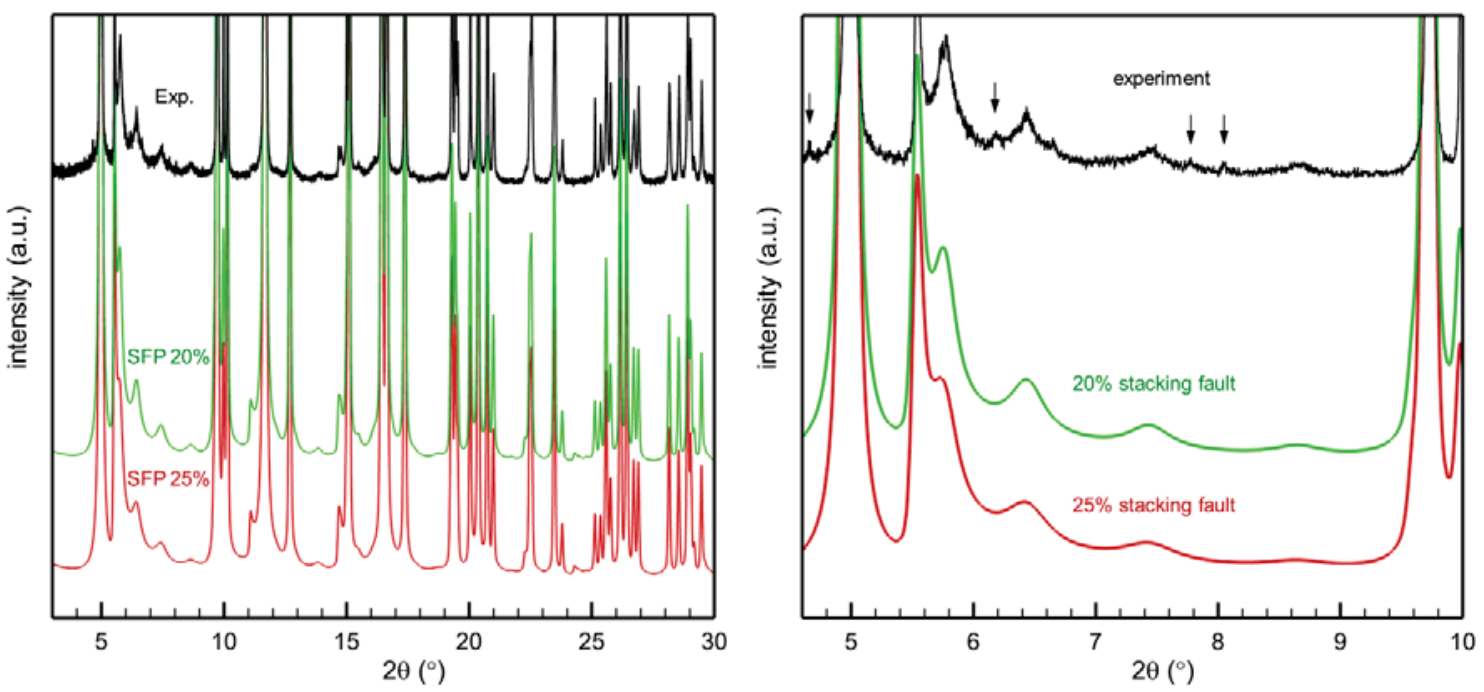

Figure 7.20 DIFFaX simulation of X-ray powder diffraction of two-phase intergrowth of $0.5 \mathrm{Li}_{2} \mathrm{MnO}_{3}-0.5 \mathrm{Li}\left(\mathrm{Ni}_{3 / 8} \mathrm{Co}_{1 / 4} \mathrm{Mn}_{3 / 8}\right) \mathrm{O}_{2}$ (Toda HE5050) with $20 \%$ and $25 \%$ stacking faults. Right panel is a detailed view of the left panel in $2 \theta$ range between $4.6^{\circ}-10^{\circ}$. Experimental profile is plotted for comparison.

Finally, Fig. 7.21 plots the DIFFaX simulated diffraction profiles of $\mathrm{Li}_{1.2} \mathrm{Ni}_{0.15} \mathrm{Co}_{0.1} \mathrm{Mn}_{0.55} \mathrm{O}_{2}$ with above determined $20 \%$ stacking faults, respectively using three different structure models (two-phase intergrowth, ordered solid solution, disordered solid solution). As discussed above, all three models produce qualitatively similar diffraction profiles as compared to the experimental profile; nevertheless, they are quantitatively different and can be distinguished by quantitative analysis. The two-phase intergrowth model consisting of ordered $\mathrm{Li}^{2 \mathrm{c}} \mathrm{Li}^{4 \mathrm{~h}}\left(\mathrm{Li}^{2 \mathrm{~b}} \mathrm{Mn}^{4 \mathrm{~g}}\right) \mathrm{O}^{4 \mathrm{i}} \mathrm{O}^{8 \mathrm{j}}$ and $\mathrm{Li}^{2 \mathrm{c}} \mathrm{Li}^{4 \mathrm{~h}}\left[\mathrm{Ni}^{2 \mathrm{~b}}\left(\mathrm{Ni}_{1 / 16} \mathrm{Co}_{3 / 8} \mathrm{Mn}_{9 / 16}\right)^{4 \mathrm{~g}}\right] \mathrm{O}^{4 \mathrm{i}} \mathrm{O}^{8 \mathrm{j}}$ and $\sim 25 \%$ stacking faults best interpret the experimental observations. This insight into the pristine Toda HE5050 will serve as a basis for subsequent study of the structural changes during charge-discharge cycles as related to lithium extraction/insertion and capacity fade phenomenon of the cathode materials. 

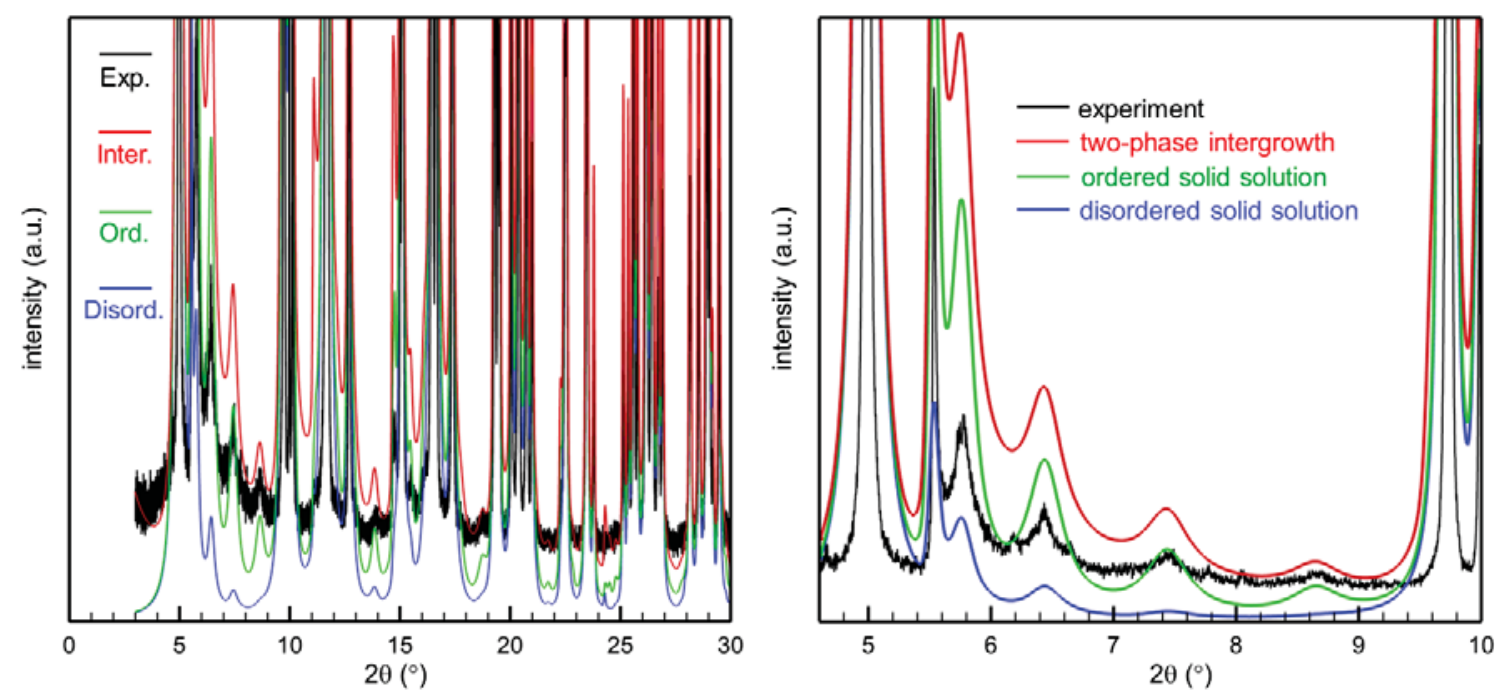

Figure 7.21 DIFFaX simulation of X-ray powder diffraction based on three different structure models (two-phase intergrowth, ordered solid solution, disordered solid solution) of $\mathrm{Li}_{1.2} \mathrm{Ni}_{0.15} \mathrm{Co}_{0.1} \mathrm{Mn}_{0.55} \mathrm{O}_{2}$ with $20 \%$ stacking faults. Right panel is a detailed view of the left panel in $2 \theta$ range between $4.6^{\circ}-10^{\circ}$. Experimental profile is plotted for comparison.

\subsubsection{Hierarchical Structural Refinement Approach Applied to HE5050}

Based on the previous section, hierarchical structural refinement approach is attempted to apply for HE5050 with layered structure notation: $0.6 \mathrm{Li}\left(\mathrm{Li}_{1 / 3} \mathrm{Mn}_{2 / 3}\right) \mathrm{O}_{2-}$ 0.4 $\mathrm{Li}\left(\mathrm{Ni}_{3 / 8} \mathrm{Co}_{1 / 4} \mathrm{Mn}_{3 / 8}\right) \mathrm{O}_{2}$. Also, Wyckoff positions are adopted for the two end members to clarify the atomic occupations $0.6\left(\mathrm{Li}^{2 \mathrm{c}} \mathrm{Li}^{4 \mathrm{~h}}\left(\mathrm{Li}^{2 \mathrm{~b}} \mathrm{Mn}^{4 \mathrm{~g}}\right) \mathrm{O}^{4 \mathrm{i}} \mathrm{O}^{8 \mathrm{j}}\right)$ 0.4 $\left(\mathrm{Li}^{2 \mathrm{c}} \mathrm{Li}^{4 \mathrm{~h}}\left[\mathrm{Ni}^{2 \mathrm{~b}}\left(\mathrm{Ni}_{1 / 16} \mathrm{Co}_{3 / 8} \mathrm{Mn}_{9 / 16}\right)^{4 \mathrm{~g}}\right] \mathrm{O}^{4 \mathrm{i}} \mathrm{O}^{8 \mathrm{j}}\right)$. As stated in section 7.2.1, Ni possesses the similar size to $\mathrm{Li}$, therefore, it is reasonable to assume that part of $\mathrm{Ni}$ occupies $2 \mathrm{~b}$ site and other part of Ni mixes with $\mathrm{Co}$ and $\mathrm{Mn}$ to form disordered constituents with specific occupancies $\left(\mathrm{Ni}_{1 / 16} \mathrm{Co}_{3 / 8} \mathrm{Mn}_{9 / 16}\right)^{4 \mathrm{~g}}$ on $4 \mathrm{~g}$ site. Fig. 7.22 compares the refinement results of two-phase intergrowth model with stacking faults and solid solution model for HE5050. It is worth noting that, even the smallest value of $R_{P}$ with homogeneous stacking fault 
distribution is achieved at probability $20 \%$, the value of $R_{P}$ is further reduced with heterogeneous distributions of stacking faults, where $~ 32 \%$ volume fraction of HE5050 exhibits $10 \%$ stacking faults, 32\% volume fraction exhibits $20 \%$ stacking faults and $36 \%$ volume fraction exhibits $90 \%$ stacking faults. Previous section concluded that introduction of $\mathrm{LiCoO}_{2}$ phase could reduce the overall stacking faults in $\mathrm{Li}_{2} \mathrm{MnO}_{3}$ phase $(\sim 40 \%)$, however, HE5050 presents more complex feature as shown in Fig. 7.22(b). Instead of further reducing overall stacking faults to below $\sim 40 \%, 90 \%$ stacking faults shows up with relatively large weight percentage $(\sim 36 \%)$. The reason for the emergence of special feature in HE5050 is that end member $\mathrm{Li}\left(\mathrm{Ni}_{3 / 8} \mathrm{Co}_{1 / 4} \mathrm{Mn}_{3 / 8}\right) \mathrm{O}_{2}$ also contributes multiple stacking vectors due to its metal layer ordering contrary to pure $\mathrm{LiCoO}_{2}$. It is also worth to mention that two-phase intergrowth model for HE5050 with homogeneous distributions of stacking faults have less ascendancy over solid solution model. As one of possible arguments, that the fitting data on pure $\mathrm{Li}\left(\mathrm{Ni}_{3 / 8} \mathrm{Co}_{1 / 4} \mathrm{Mn}_{3 / 8}\right) \mathrm{O}_{2}$ is not available as $\mathrm{LiCoO}_{2}$, needs to be compromised for our construction of two-phase model, resulting in potential deviation from true values. Furthermore, transition layer ordering patterns for $\mathrm{Li}\left(\mathrm{Ni}_{3 / 8} \mathrm{Co}_{1 / 4} \mathrm{Mn}_{3 / 8}\right) \mathrm{O}_{2}$ [148] are still under investigation, leading to another variant for possible deviation. The settle for these issues could further improve the accuracy for our hierarchical structural refinement approach to stacking fault analysis. 


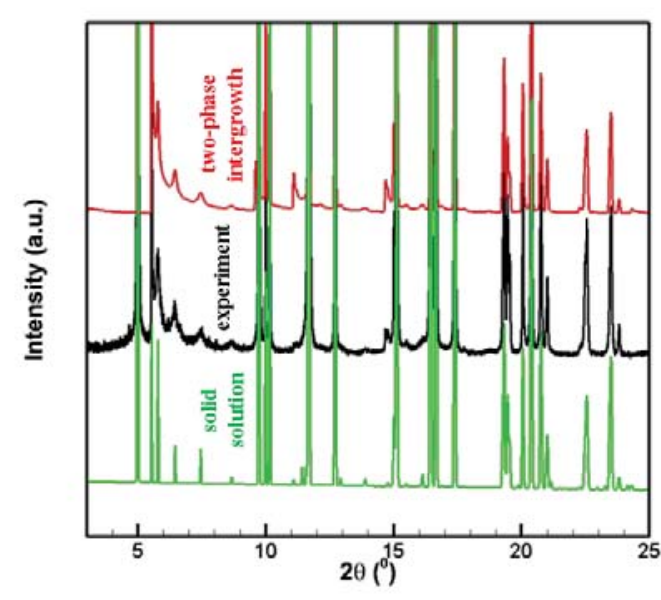

(a)

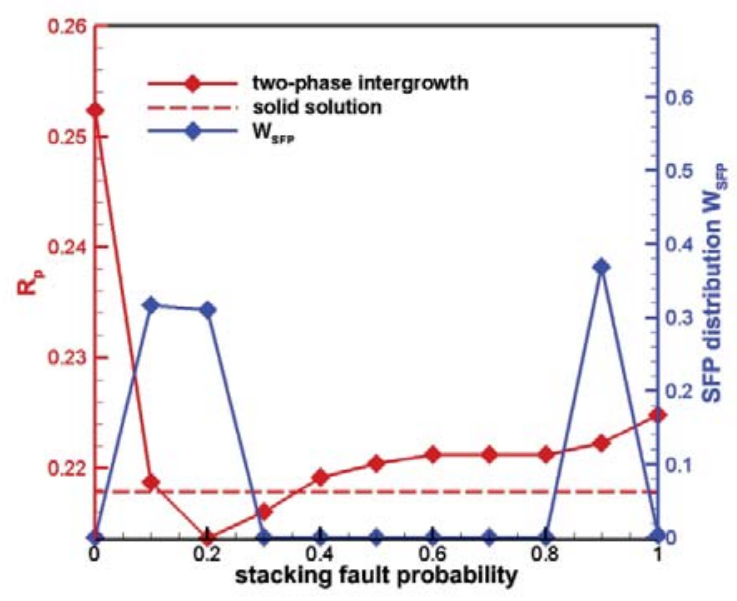

(b)

Figure 7.22 Comparison of two-phase intergrowth model with stacking faults and solid solution model for $50 \% \mathrm{Li}_{2} \mathrm{MnO}_{3}-50 \% \mathrm{Li}\left(\mathrm{Ni}_{3 / 8} \mathrm{Co}_{1 / 4} \mathrm{Mn}_{3 / 8}\right) \mathrm{O}_{2}$. (a) Powder diffraction intensity profiles of experiment, solid solution model, and two-phase intergrowth model with heterogeneous distributions of stacking faults. (b) Fitting residual error $R_{P}$ of solid solution model and two-phase intergrowth model with different homogeneous stacking fault probabilities.

\subsection{Faulting in Oxygen Framework and Vacancies}

\subsubsection{Oxygen Faulting Model in DIFFaX}

As previously mentioned layered structure, faulting effects usually present in the form of atom ordering in the transition metal layer when applying specific translational stacking vectors, while oxygen layers keep ABC stacking unchanged as Fig. 7.23 shown to us. Ideal monoclinic angle $\beta$ equals to $\arccos (-1 / 3) \approx 109.4712^{0}$ based on close packed geometry. Fig. 7.24 shows the allowed stacking vectors to recover oxygen layer $\mathrm{ABC}$ sequences. All the dotted arrows originate from the common reference joint point located 
at bottom layer (sequence A) and point to the geometrical allowed positions located at top layer (sequence C) after translation. There are total seven stacking vectors as shown in Fig. 7.24 , however, only part of them is independent relying on the ordering of metal layers. For example, if metal layer is totally disordered, then, only one stacking vector is independent $\left([0,0,1]_{\text {mon }}\right)$, additionally, if metal layer is ordered in the way as transition metal layer in $\mathrm{Li}_{2} \mathrm{MnO}_{3}$, three out of seven stacking vectors are independent $\left([0,0,1]_{\text {mon }}\right.$ $\left.[0,1 / 3,1]_{\operatorname{mon}}[0,-1 / 3,1]_{\operatorname{mon}}\right)$.

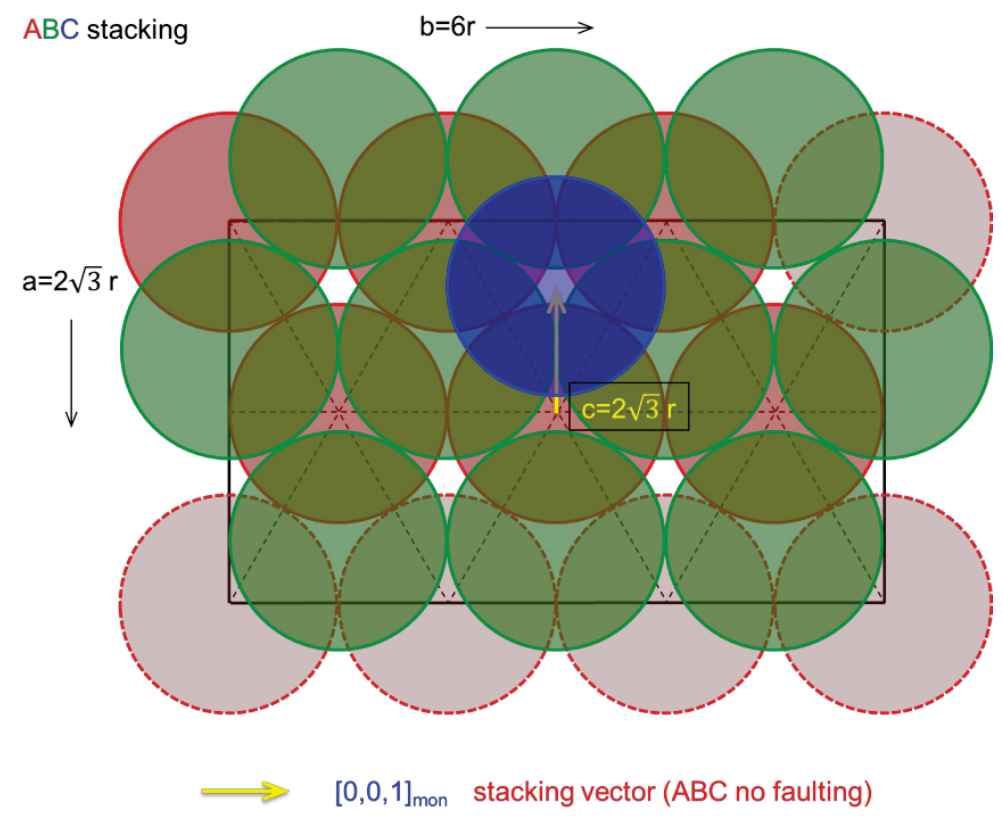

Figure 7.23 $\mathrm{ABC}$ non-faulting stacking for oxygen layers. Yellow arrow represents monoclinic c axis, while black arrows represent monoclinic a, b axes. Letter $r$ designates radius of oxygen. Layer with red spheres locates at the bottom (A), layer with green spheres locates at the middle (B) and layer with blue spheres locates at the top (C). 


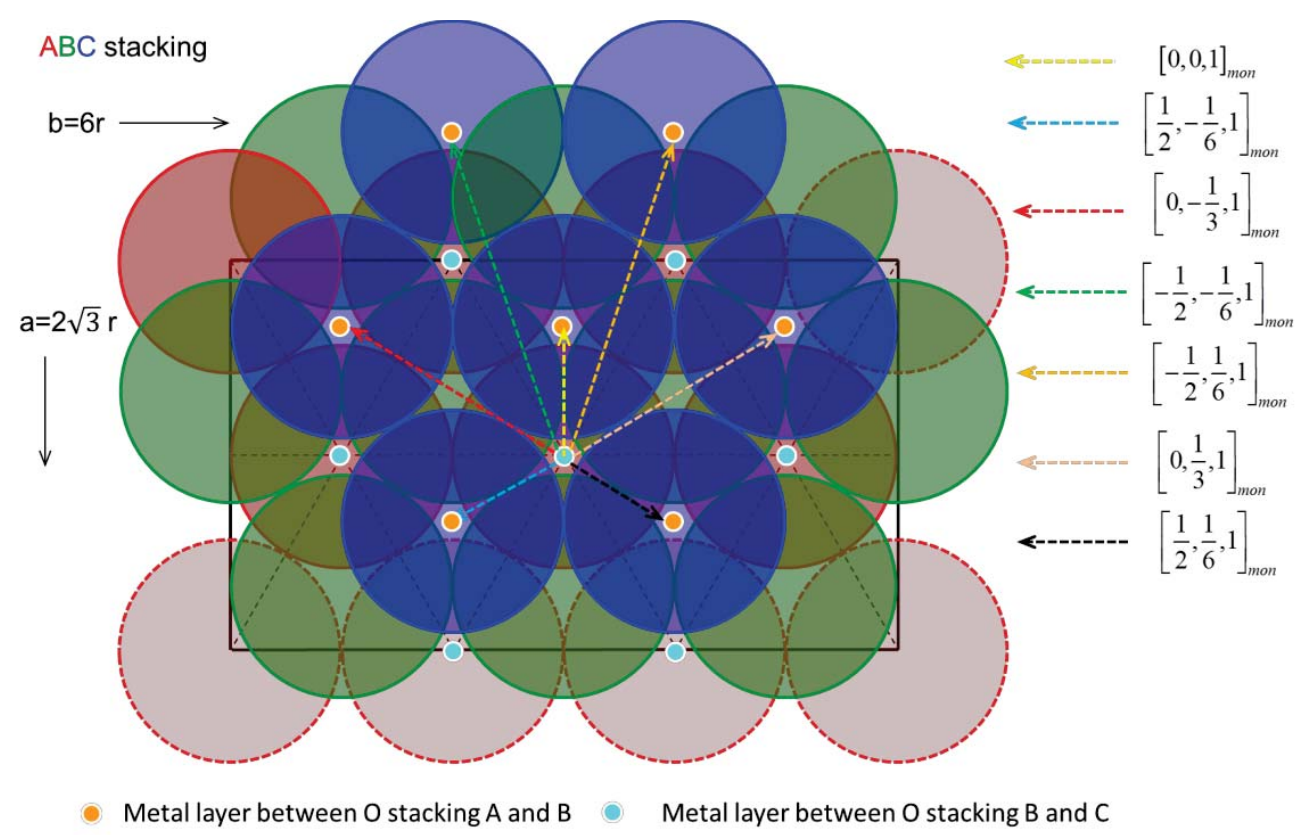

Figure 7.24 Possible stacking vectors are designated with colorful dotted arrows corresponding to different stacking translations in monoclinic notation, which keep oxygen framework ( $\mathrm{ABC}$ stacking) unchanged. Orange spheres represent metal layer located between oxygen layer sequences $\mathrm{A}$ and $\mathrm{B}$, light blue spheres represent metal layer between oxygen layer sequences B and C.

All the discussions above are about the stacking geometries without oxygen framework change. It is essential to ask what if oxygen layers engender faulting. As shown in Fig. 7.25, instead of keeping strict $\mathrm{ABC}$ stacking sequence, the third oxygen layer is translated to one of such three places labelled with red " $\mathrm{X}$ " which violate the traditional $\mathrm{C}$ type stacking. Therefore, three possible stacking vectors leading to oxygen layers faulting are $[1 / 3,0,1]_{\text {mon }},[-1 / 6,-1 / 6,1]_{\text {mon }},[-1 / 6,1 / 6,1]_{\text {mon. In order to capture the oxygen layers }}$ faulting feature in DIFFaX model, special unit cell is constructed as shown in Fig. 7.26(b). When constructing the cell to describe effect of oxygen layers faulting, metal layers should be compatibly addressed to well fit in the inter oxygen layer. It is worth to note that $\mathrm{Li}$ 
atoms deviate accordingly with oxygen layer faulting as compared with non-faulting cell shown in Fig. 7.26(a). Such the cell setting up facilitates the investigation of faulting sequence of $\underline{\mathrm{AB}} \underline{\mathrm{CB}} \underline{\mathrm{CB}} \underline{\mathrm{CB}} \underline{\mathrm{CA}} \cdots$ compared with perfect stacking $\underline{\mathrm{AB}} \underline{\mathrm{CA}} \underline{\mathrm{BC}} \underline{\mathrm{AB}} \underline{\mathrm{CA}} \cdots$. If M layer is totally disordered, only one oxygen layers faulting stacking vector is independent (e.g. $[1 / 3,0,1]_{\mathrm{mon}}$ ) as demonstrated in Fig. 7.26(c). While, if M layer is ordered as transition layer in $\mathrm{Li}_{2} \mathrm{MnO}_{3}$, additional degrees of freedom concerning ordering (three stacking vectors as stated before) combined with three oxygen layers faulting stacking vectors together give 9 possible stacking sequences, however, only 3 are independent listed here as $[1 / 3,0,1]_{\mathrm{mon}},[1 / 3,1 / 3,1]_{\mathrm{mon}},[1 / 3,-1 / 3,1]_{\mathrm{mon}}$. More importantly, interchange Li layer and M layer in the cell will give different stacking structure, thus, different results, because the alternative order of Li and M stacking is changed. Such argument could be further addressed by adding more cells in the model to account for specific purpose.

Furthermore, previous constructed cell does not completely account for all the oxygen layer faulting features. Fig. 7.27 shows another way of cell construction to additionally consider oxygen layer faulting features. As shown in Fig. 7.27(b), newly constructed cell is of different stacking vector c' instead of previously perfect stacking monoclinic c axis inclining to form "mirror plane" (001) mon. Compared to previous stacking sequence, the new feature of oxygen layer stacking fault could be involved in as $\underline{\mathrm{AB}} \underline{\mathrm{CB}} \underline{\mathrm{AC}} \underline{\mathrm{BC}} \underline{\mathrm{AB}} \cdots$ which could not be reproduced with former cell construction only, in the other words, in the current frame of DIFFaX, multiple cells concerning different feature of oxygen layer faulting and the corresponding stacking vectors are required to combine 


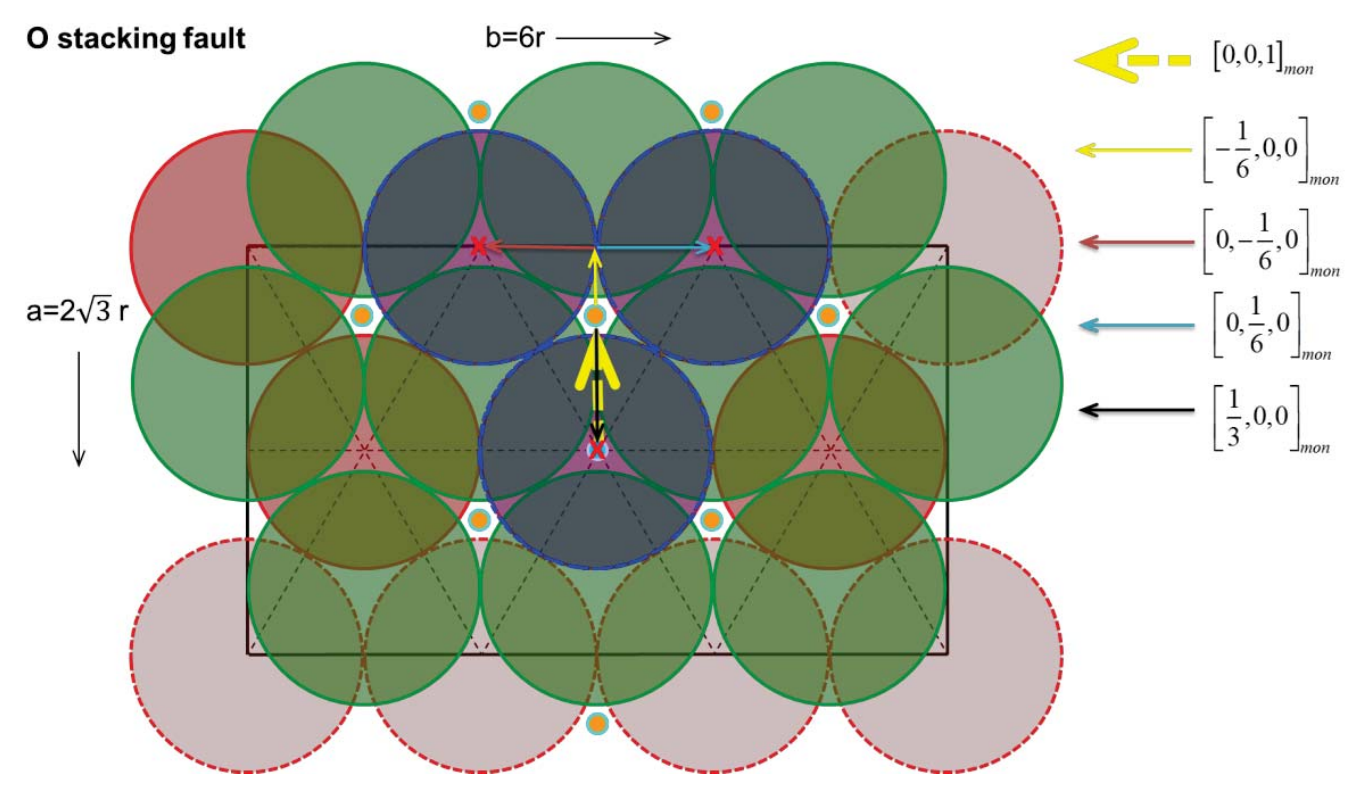

Figure 7.25 Oxygen layer faulting. Red cross symbols " $\mathrm{X}$ " represent possible positions for the third oxygen layer violating $\mathrm{ABC}$ stacking sequence. Arrows with variant colors are adopted to designate translations, the combinations of which are used to obtain the positions labelled with " $\mathrm{X}$ " after translation originated from oxygen bottom layer (A).

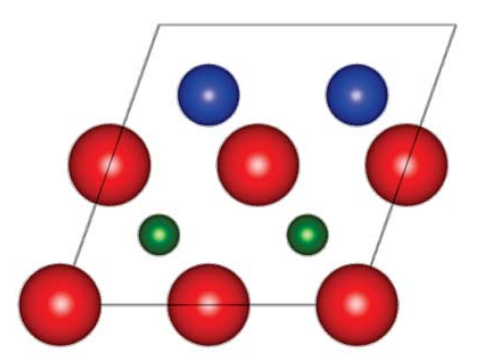

(a)

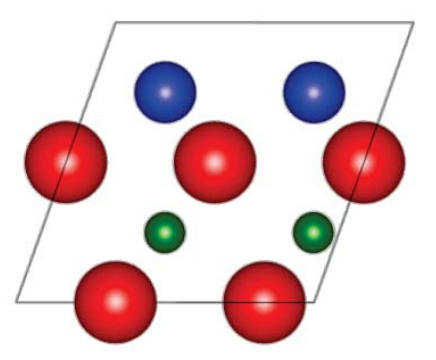

(b)

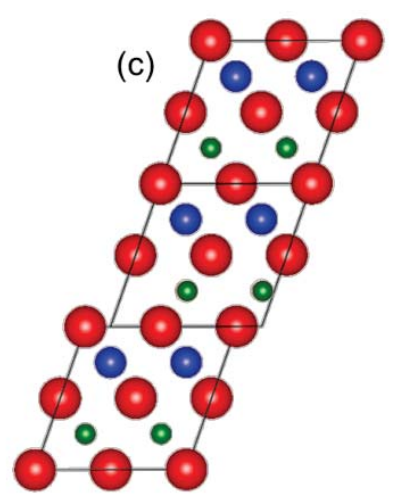

Figure 7.26 Projective views along monoclinic b axis (a) unit cell for perfect stacking with [001] mon stacking vector. (b) unit cell constructed for revealing oxygen layer faulting structure with possible stacking vectors $[1 / 3,0,1]_{\mathrm{mon}},[-1 / 6,-1 / 6,1]_{\mathrm{mon}}$, and $[-1 / 6,1 / 6$,

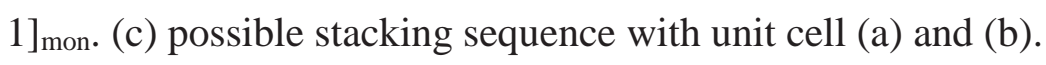

together to fully consider more complex oxygen layer faulting geometry. 


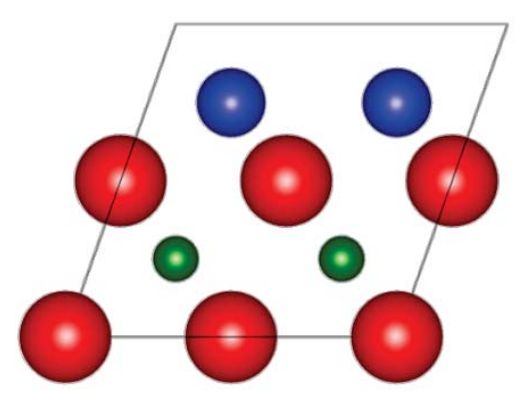

(a)

* Li

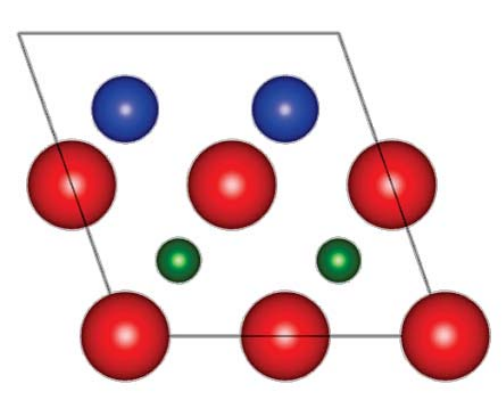

(b)

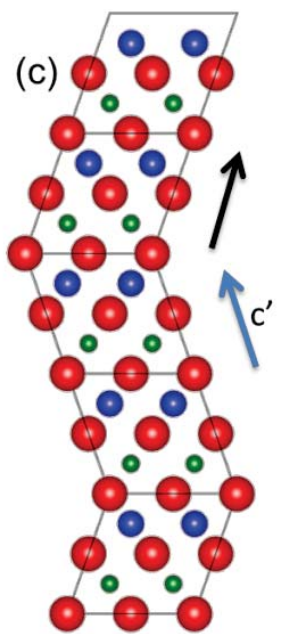

Figure 7.27 Projective views along monoclinic b axis (a) unit cell for perfect stacking with [001] mon stacking vector. (b) unit cell constructed for revealing another oxygen layer faulting structure with c' stacking vector. (c) possible stacking sequence with unit cell (a) and (b).

\subsubsection{Extraordinary X-ray Diffraction Phenomenon due to Oxygen Faulting}

To perform DIFFaX calculation, input parameters are given as following: $\lambda=1.5418 \AA$; Pseudo-Voigt parameters $\mathrm{GU}=0.015, \mathrm{GV}=0.015, \mathrm{GW}=0.0034$ and $\eta=0.63$; Lattice parameters $\mathrm{a}_{\mathrm{mon}}=4.9292 \AA, \mathrm{b}_{\text {mon }}=8.537625 \AA$ and $\mathrm{c}_{\mathrm{mon}}=4.9292 \AA$. All atoms occupy ideal positions. As one type of oxygen layer stacking faults shown in Fig. 7.26, prototypical system $\mathrm{LiCoO}_{2}$ is first adopted for investigation. It is worth to mention that only two stacking vectors $[0,0,1]_{\mathrm{mon}}$ and $[1 / 3,0,1]_{\mathrm{mon}}$ are required to fully consider such oxygen layer faulting type due to the total disorder in both $\mathrm{Li}$ and Co layers. Different percentages of oxygen layer faulting are calculated and summarized in Fig. 7.28. As guided by black dotted line on Fig. 7.28 , it is interesting to note that peak position around $2 \theta \sim 45^{\circ}$ at $0 \%$ oxygen layer faulting gradually drifts away towards lower $2 \theta$ angle when increasing 
oxygen layer faulting probability, while peak profile first shows broadening feature and then become sharp again at high enough oxygen layer faulting probability. Dotted red rectangles shown in Fig. 7.28 are used to highlight the emergence of new peaks when oxygen layer faulting reaches to a relatively high value.

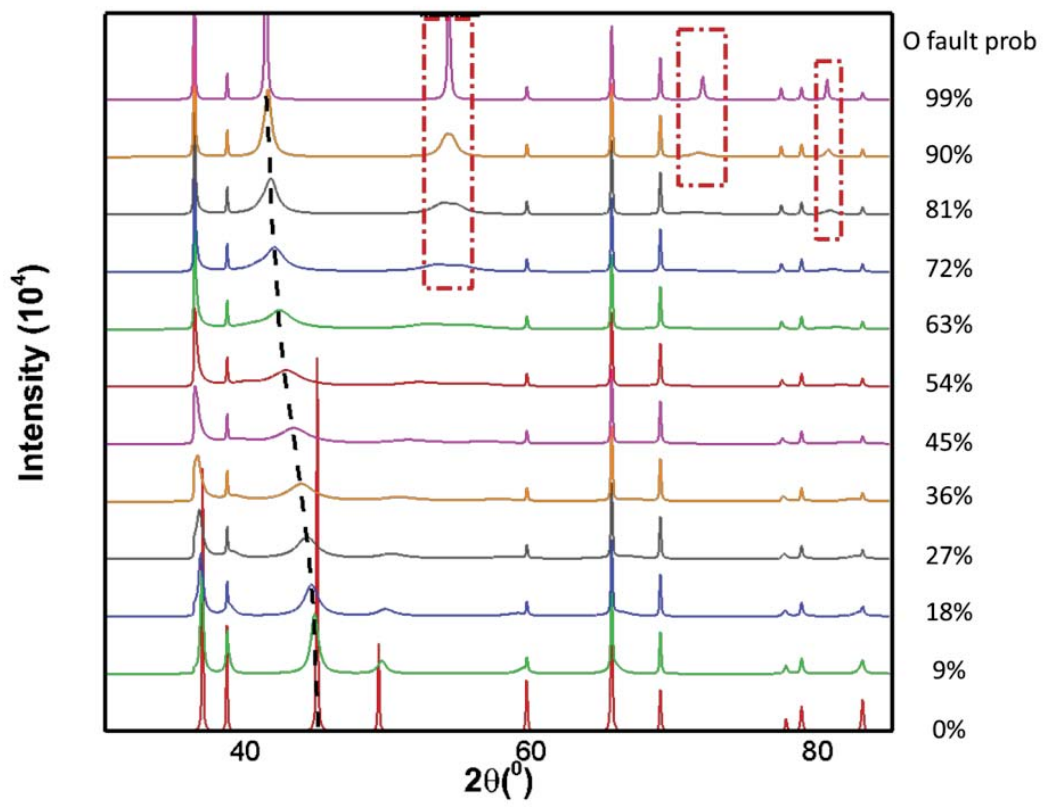

Figure 7.28 Total 12 calculated X-ray intensity curves with different oxygen layer faulting probabilities (ranging from 0\% 100\%) are presented (labelled on the right side). Black dotted line is used to guide to eyes about peak position drifting around $2 \theta \sim 42^{0}$ when increasing oxygen layer faulting probability. Three red dotted rectangles are adopted to highlight the emergence of new peaks at high percentage of oxygen layer faulting.

To further exploit oxygen layer faulting feature with ordering in transition layer, system $\mathrm{Li}_{2} \mathrm{MnO}_{3}$ is qualified candidate to involve in more stacking vectors to account for additional variables due to in-plane ordering. Therefore, total 4 stacking vectors $[0,0,1]_{\text {mon, }}$, $[1 / 3,0,1]_{\text {mon }},[1 / 3,1 / 3,1]_{\text {mon }}$ and $[1 / 3,-1 / 3,1]_{\text {mon }}$ are required to fully consider such oxygen layer faulting case with ordering. In order to emphasis the oxygen layer faulting effect on 
diffraction patterns, a contrast group with merely metal layer faulting (no oxygen layer faulting) is constructed as comparison purpose. To be consistent, contrast group uses the same sequence of stacking faulting probability as shown in Fig. 7.29. As expected, oxygen layer faulting still causes the "drifting" effect around the $2 \theta$ angle $42^{0}$ in $\mathrm{Li}_{2} \mathrm{MnO}_{3}$ ordered system. Black dotted line and red rectangles in Fig. 7.29 play the same function as in Fig. 7.28 to highlight the features for oxygen layer faulting. Compared with traditional metal layer faulting only, oxygen layer faulting presents extraordinary phenomenon such as peak shift around $42^{0}$ and emergence of new peaks in particular positions. Such evidence is critical to characterize new materials with initial structural guess whether it contains oxygen layer faulting.

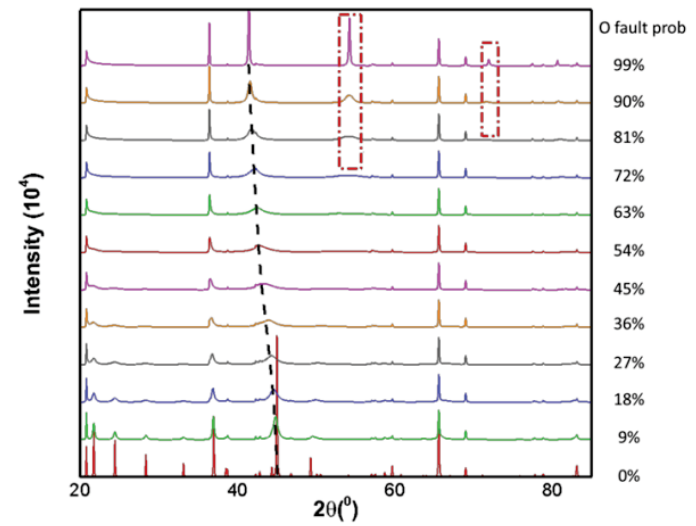

(a)

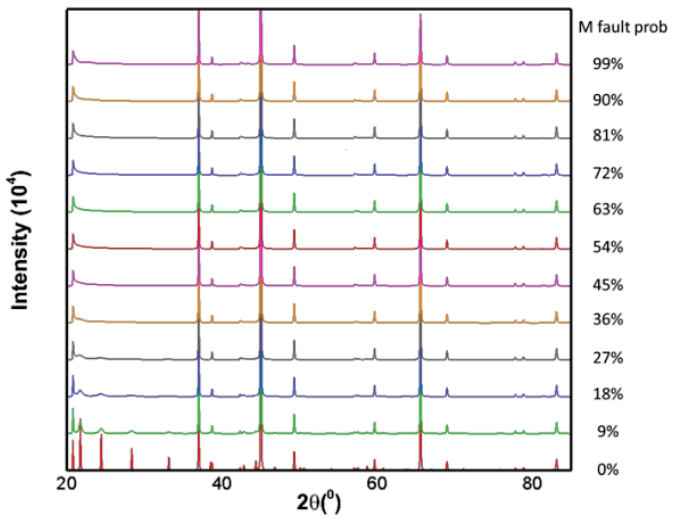

(b)

Figure 7.29 Total 12 calculated X-ray intensity curves for (a) Oxygen layer fault (b) Metal layer faulting with different stacking probabilities (ranging from 0\% 100\%). Black dotted line in (a) is used to guide to eyes about peak position drifting around $2 \theta \sim 42^{0}$ when increasing oxygen layer faulting probability. Two red dotted rectangles in (a) are adopted to highlight the emergence of new peaks at high percentage of oxygen layer faulting. 
As shown in Fig. 7.27 indicating another way for generating oxygen layer faulting, "anti-phase" like faulting structure motivates us to tentatively call it anti-phase oxygen layer faulting. For simplicity, system $\mathrm{LiCoO}_{2}$ is used to avoid the potential complexity for ordering. Fig. 7.30 shows the calculated X-ray diffraction curves for such anti-phase oxygen layer faulting with variant faulting probabilities. For illustration purpose, comparison is made between Fig. 7.30 and Fig. 7.28 of different type of oxygen layer faulting. Around $2 \theta \sim 37.5^{0}$, both types of oxygen layer faulting show split doublet peaks. Such doublet peaks present more diffused feature for anti-phase oxygen layer faulting case in Fig. 7.30, while in Fig. 7.28, doublet peaks show relatively sharp profiles which could be distinguished clearly. In addition, anti-phase oxygen layer faulting case presents more peculiar peak shapes in this $2 \theta$ range. In the $2 \theta$ range from $45^{\circ}$ to $50^{\circ}$, original X-ray pattern without oxygen layer faulting shows two separated peaks. For anti-phase oxygen layer faulting, increasing percentage of faults will at first lead to the gradual disappear of the peak intensities and later the gradual appearance of such peak intensities as circled with red dotted rectangle in Fig. 7.30 when fault reaches to certain high value $\sim 72 \%$. However, another type of oxygen layer faulting does not present such feature as shown in Fig. 7.28 with merely rapid disappear of peak intensities after $18 \%$ oxygen layer faulting probability. $2 \theta \sim 54.2^{0}$ processes no peak for anti-phase oxygen layer faulting while for another oxygen layer faulting type, new peaks are generated when the fault percentage exceeds $72 \%$ as circled out in Fig. 7.28. In addition, anti-phase oxygen layer faulting does not have the peak drifting feature around $2 \theta \sim 45^{0}$. 


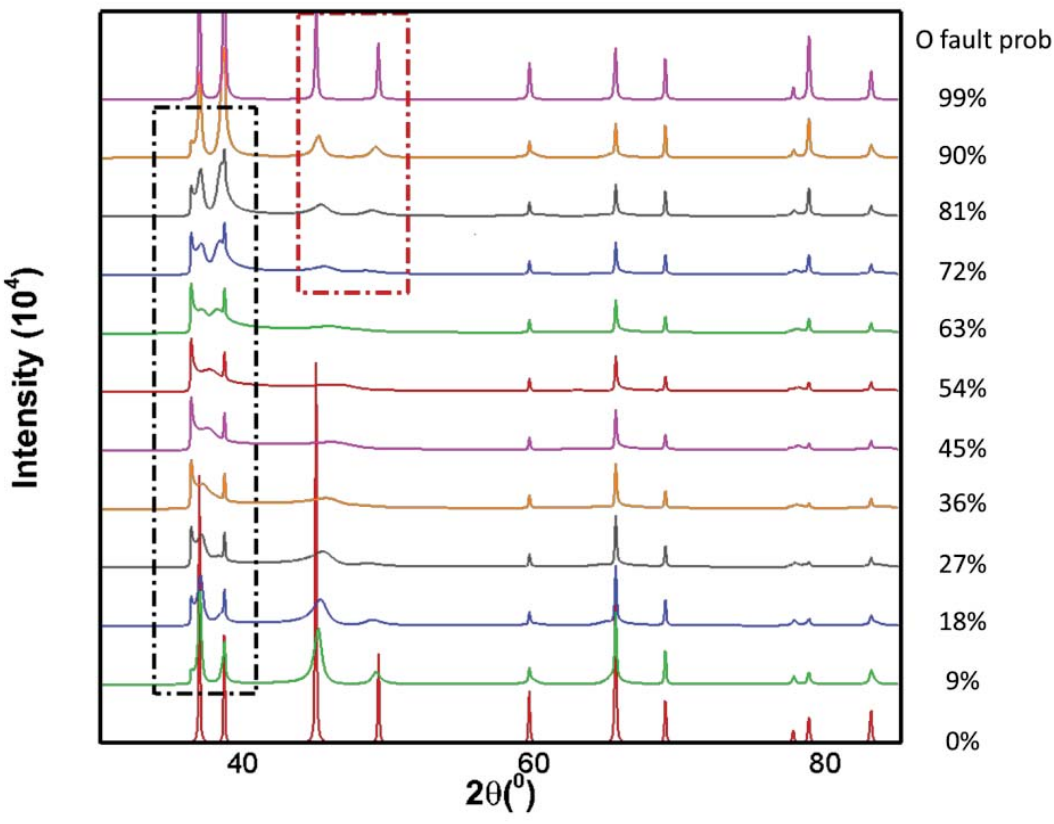

Figure 7.30 Total 12 calculated X-ray intensity curves with different "anti-phase" oxygen layer faulting probabilities (ranging from 0\% 100\%) are presented (labelled on the right side). Black dotted rectangle is used to circle out feature peaks when varying oxygen layer faulting probability. Red dotted rectangle is adopted to highlight the emergence of new peaks at high percentage of "anti-phase" oxygen layer faulting.

\subsubsection{Treatment for Voids}

For the investigation purpose of dynamic process during extraction/insertion of lithium in battery cathode, generation of voids in the layered structure is important and needs to be purposely addressed. DIFFaX supplies us with already good enough platform that only small "tricky" modifications based on it could well realize the way to do voids dynamic process study. Void as its literally meaning is interpreted to be "nothing there". The treatment for the void in DIFFaX software assembly is to modify the database: "data.sfc" (may vary in later versions) containing scattering parameters for all the elements. New element called "VOID" is added into the database as a row with setting all the 
scattering parameters to be zero. The only thing you need to pay attention to is taking care

of filling the blank space between parameters with required whitespace only. Then, voids could be treated as normal element with designed positions based on specific requirement.

\subsubsection{Conclusion}

In this section, layer by layer structural oxygen layer faults are studied by seamlessly constructing oxygen layer faulting unit cell models and performing DIFFaX simulations. The method has a capability to capture the structural information caused by oxygen layer faulting which is quite different from traditional M (transition metal) faulting. Based on the geometry consideration, two types of oxygen layer faulting are proposed. As prototypical systems, $\mathrm{LiCoO}_{2}$ and $\mathrm{Li}_{2} \mathrm{MnO}_{3}$ are adopted to show the oxygen layer faulting effects on X-ray diffraction pattern. Without the complexity of in-plane ordering, X-ray diffraction calculation for $\mathrm{LiCoO}_{2}$ is performed to study the pure effects coming from different types of oxygen layer faulting only. It is found that anti-phase oxygen layer faulting type will lead to peculiar shape of certain peaks around $2 \theta \sim 37.5^{0}$ and special peak intensity changes with fault percentage around $2 \theta \sim 54.2^{0}$. Another oxygen layer faulting type results in the peak "drifting" around $2 \theta \sim 45^{0}$ and emergence of new peaks at specific $2 \theta$ angles. In addition, $\mathrm{Li}_{2} \mathrm{MnO}_{3}$ with one type of oxygen layer faulting is calculated by extra consideration of ordering. It is found that incoherent effects separately from M layer faulting and oxygen layer faulting and coherent effects from their coupling contribute to the diffraction pattern together showing superimposed features. The proposed oxygen layer faulting models provide a feasible approach to characterize the structure related with oxygen layer faulting by identifying the extraordinary peaks in layer by layer battery 
materials. Such structural information with oxygen layer faulting could be potentially linked with materials performances. In addition, the treatment for voids in DIFFaX supplies us a feasible way to investigate dynamic structural changes during battery cycling accompanied by extraction/insertion of lithium ions. 


\section{Chapter 8. Summary and Prospective Research}

This thesis aims at studying the microstructure-property relations in ferroelectric materials and lithium ion cathode materials. Mesoscale phase field model is exploited for ferroelectric materials with special focuses on extrinsic domain properties which could be tailored by external loadings and microstructure features such as grain shape, textures and two phase composites. Atomic scale calculations concerning atomic occupancy and crystal layered structure for lithium ion cathode materials are conducted with DIFFaX. With proposed algorithms, the resultant X-ray spectrums are appropriately analyzed in order to quantitatively extract the structural information by comparing with experimental data. All the results and insights are summarized and prospective research is discussed as well in this chapter.

\subsection{Summary of Results from Computational Studies}

\section{Ferroelectric Materials:}

As prototypical ferroelectric materials, PZT single crystals are first investigated by performing both phase field simulations and theoretical analysis. The results show that external electric field could tailor domain patterns by adjusting its magnitude and applied direction so that polarizations accommodate themselves to align to the energy favorable position. By further taking into consideration of easy axes for polarizations in different phases, electric field is applied along [111] direction to obtain slant stripe domains forming 
(110) domain walls in tetragonal phase, while in rhombohedral phase, electric field is applied both along [100] and [110] directions which could result in stripe domain with (100) twins and slant stripe domains with (110) twins respectively. It is worth to mention that more general and interesting domain pattern such as herringbone could emerge from application of [100] electric field in rhombohedral phase. Apart from domain pattern design with external electric field, simulations also show that domain size could be controlled by temperature and electric field magnitude along non-polar direction. In PZT tetragonal phase, electric field is applied along [111] direction when quenching system to room temperature below Curie point. It is found that minimal domain size and highest domain wall density are obtained at intermediate electric field magnitude, while lower and higher fields produce coarser domains thus lower domain wall densities. As another important factor in domain size control, temperature impacts on domain size through the way that relatively higher quenching temperature could flatten the free energy landscape, making easier selection of polar nuclei by external electric field thus shifting moderate electric field magnitude at which the smallest domain size is obtained to the lower value. It indicates that appropriate combination of electric field magnitude, direction and temperature can provide optimal processing conditions for crystallographic domain engineering. In addition, nucleation stage also serves as an important part to reveal domain formation mechanisms. Therefore, theoretical study on ferroelectric single crystals is conducted to show that, differing from classical short range nucleation, the special way for ferroelectrics to nucleate is to form long range correlated patterns in order to assembly circumvent high energy barrier. As a complimentary research for ferroelectric single crystal, better 
understanding of nucleation mechanisms from the theoretical way potentially supplies more feasible paths to well control and improve domain engineering technique.

In majority of time, single crystals such as PZT are intractable due to fabrication difficulties. Traditional polycrystals with their grains randomly orientated only present isotropic properties; therefore, in order to develop similar anisotropic properties as single crystals, techniques to generate texture for polycrystals are required. As one of the mostly applied techniques, templated grain growth (TGG) is used to emerge uniaxial texture for ferroelectric polycrystals. Phase field model is constructed for TGG to take care of its special features differing from common grain growth. X-ray diffraction calculation is then performed during grain growth to instantaneously capture texture development characterized by Lotgering factor. Complementary experiment is conducted by our collaborators to validate our model. The simulation shows that it is average distance between template seeds that determine the final texture rather than template volume fraction itself. Relatively smaller distance between template seeds could result in more area of well orientated grains with higher texture because adjacent grains are easier to grow to contact with each other. Such findings supply the possible way to optimize the material cost-performance ratio with TGG technique. Consequently, as one of the accompanied issue from TGG, templated grain shape usually exhibits anisotropy. Computational study reveals that ferroelectric response along direction with higher grain boundary density (direction along minor axis of oblate grain) is lower than the direction with smaller grain boundary density. The reason is due to the existence of more domain variants tolerated by relatively looser grain boundaries, which facilitate higher ferroelectric response. As 
compared with texture effect, grain shape only plays the minor role in ferroelectric response. Texture-performance curves are constructed with variant distribution angle (deviation angle from [001] axis for uniaxial alignment) to quantitatively study the texture effect. Simulations show that remnance, coercivity and piezoelectricity present non-linear monotonic decrease with distribution angle, while dielectric constant increases with distribution angle. An interesting phenomenon that the texture-performance curves corresponding to remnance, coercivity and dielectric constant show saturation behavior after distribution angle $60^{\circ}$ demonstrates the insensitive zone of the texture-performance relations for PZT ferroelectric materials. As another accompanied issue that template seeds in TGG are usually single crystals of second inferior phase which cause "composites" effect due to multi-phase coexistence, such effect necessitates the further investigations to illustrate the competition mechanisms between "composites" effect and texture development. The computational results demonstrate the electric and elastic interactions on the two phase interface which could highly affect domain morphology during polarization switching resulting in a softening remnance and coercivity compared with pure primary phase. Calculated curves of second phase volume fraction against ferroelectric properties prove the existence of critical volume fraction $\sim 1 \%$ which quantitatively describes the competition mechanisms between the capacity of texture development for the chosen volume fraction and its role as second phase inclusion. To summarize, computational exploration on textured ferroelectric polycrystals facilitates the realization of tailoring materials properties through better control of microstructures based on the understanding of underlying mechanisms. 


\section{Lithium Ion Cathode Materials:}

As another functional material which is also characterized by microstructure, lithium ion cathode material is studied with open source package DIFFaX to explore its structural information such as ordering and stacking fault in the atomic scale. One of the main features in structure of layer by layer intergrowth is stacking fault distribution to which the phenomenon that certain constituent composition presents high cycling performance could be possibly attributed. In order to circumvent the disadvantage that DIFFaX could only qualitatively capture stacking fault information, a hierarchical algorithm is first developed to search for stacking fault information through fitting the calculated databases to experimental data. Concretely, the databases are obtained from DIFFaX calculations by varying stacking fault probability under the framework of twophase intergrowth. Such searching algorithm is applied to serial objective materials $x \mathrm{LiCoO}_{2}-(1-x) \mathrm{Li}_{2} \mathrm{MnO}_{3}$. To construct databases for these systems, the input parameters for the two pure end members $\mathrm{LiCoO}_{2}$ and $\mathrm{Li}_{2} \mathrm{MnO}_{3}$ are required for DIFFaX. Rietveld refinement (GSAS) is adopted to obtain such required parameters for the two end members by fitting to the experimental data of $\mathrm{LiCoO}_{2}$ and $\mathrm{Li}_{2} \mathrm{MnO}_{3}$ accordingly. A weight factor is assigned for each curve with specific stacking fault probability in databases. Then, searching algorithm will return the convergent normalized weight distributions which give the best fit to objective experimental data to reflect the relative percentage of specific type of stacking fault existing in objective materials. Serial objective materials $x \mathrm{LiCoO}_{2}-(1-$ $x) \mathrm{Li}_{2} \mathrm{MnO}_{3}$ with $x=0,0.1,0.3,0.5,0.7,0.9,1.0$ are analyzed to obtain stacking fault distributions for each composition. Based on the analysis, it is confirmed that two-phase 
intergrowth model still gives better fit than solid solution model. Interestingly, for composition $x=0.5$, a special possible stacking sequence is proposed which is potentially an explanation for such composition being of the best performance. Based on the DIFFaX modeling and the proposed hierarchical algorithm, new high performance material pristine Toda HE5050 (TODA America, Inc. and Argonne National Lab) with the nominal composition form $\mathrm{Li}_{1.2} \mathrm{Ni}_{0.15} \mathrm{Mn}_{0.55} \mathrm{Co}_{0.1} \mathrm{O}_{2}$ is studied. In order to first clarify the argument that whether such layered material is solid solution or two-phase intergrowth, DIFFaX models corresponding to each of the notations are constructed for materials $0.5 \mathrm{Li}_{2} \mathrm{MnO}_{3}$ 0.5 $\mathrm{Li}\left(\mathrm{Ni}_{3 / 8} \mathrm{Co}_{1 / 4} \mathrm{Mn}_{3 / 8}\right) \mathrm{O}_{2}$ (Toda HE5050). In particular, feature peak intensities acting as "finger print" are selected based on specific $2 \vartheta$ ranges with the intended consideration on the peak sensitiveness to structural changes. In order to create "microstructure identities", feature peak intensity ratio is calculated to further distinguish between different DIFFaX models and experiment result. Computational outcomes show that two-phase intergrowth notation possesses the closest identity value to experiment, indicating that the structure in Toda HE5050 is rather two-phase intergrowth than solid solution. Sequentially, the similar numerical searching process with our hierarchical algorithm is applied to Toda HE5050 material as well to obtain its stacking fault distributions. Additionally, Oxygen layer stacking fault model and the method to treat voids in DIFFaX are proposed to further deal with more extraordinary phenomenon related with special microstructure. 


\subsection{Prospective Research}

\section{Improvement on phase field model}

For phase field model adopted in this thesis, elastic homogeneity but structural inhomogeneity is assumed to study the complex microstructures of domains, template grains, and composites. Elastic homogeneity assumption facilitates the code implementation and calculation efficiency without losing important physics insights. In our common simulated system, for example, two-phase composites, the elastic modulus mismatch between the two phases is not large, therefore, elastic homogeneity assumption is enough to give the correct behaviors with predetermined accuracy. If the system with quite large modulus contrast among multi-constituents becomes our study objective, more sophisticated model [110] by integrating elastic inhomogeneity is required to correctly study elastic couplings. Such modified model will not only extend our suitable investigation systems but also potentially improve simulation accuracy. In addition, isotropic domain wall properties are assumed, which is reflected by using isotropic gradient coefficients $\left(\beta_{\mathrm{ijkl}}=\beta\right)$ in our current phase field model. With such isotropic assumption, kinetic properties for domain wall are efficiently captured by ignoring detailed domain wall morphology. The macroscopic ferroelectric properties related with extrinsic domain wall motion are well obtained by our simulations. If domain wall morphology itself becomes study topic, anisotropy gradient coefficients [217] which will change domain wall morphology, needs to be considered. Phase field simulations on domain wall with anisotropy property could further be compared with first-principle calculations [218] in

order to study the detailed static domain wall structures, which also facilitates the study of 
nano-scale flexoelectric effect [219] in ferroelectrics. In addition, compared with current model with isotropic domain wall properties, the future modified model with improved static domain wall morphology will potentially increase the accuracy for kinetic properties of domain walls. Such improvements further facilitate the investigation on the most applicable two-phase composites in which polymer phase plays a crucial role to overcome the brittleness of another phase.

\section{Linkage between domain structure and x-ray diffraction pattern: further pavement to study innovative phenomenon}

In ferroelectric materials, due to electrostriction effect, domains are usually accompanied with lattice distortion (e.g. after cubic to tetragonal phase transformation). If domain size shrinks to nano-size, adaptive diffraction phenomenon [206] will occur. Such

phenomenon will contribute to new diffraction features in addition to the simple consideration of grain orientations. This facilitates the investigation for the domain structures through X-ray diffraction analysis. Furthermore, non-linear optical properties in ferroelectric materials are usually related with local polarization. It is motivated to obtain the ferroelectric optical properties numerically from domain structure, which broadens the research area of our simulation.

\section{Dynamic lithium extraction/insertion process and more stacking modes: expand the capability of DIFFaX}

During battery cycling process, lithium ions are extracted from interlayer spaces. Naturally, voids are generated with structural changes. Dynamic process during cycling is very important to further enhance battery performance. Detail information about the 180 
instantaneous generation of voids and accompanied structural change during cycling process helps us to identify the ion transportation pathways which are highly related with diffusivity. If we treat voids as special element, DIFFaX could supply us a way to investigate voids kinetics during battery cycling. The calculated hypothetical void configurations at different stages could be compared with in-situ X-ray diffraction data, facilitating us to resolve the structural change information during lithium ion transportation process. In addition, as we have discussed in Chapter 7, except for metal layer faulting, oxygen layers could happen faulting as well. Some peculiar X-ray diffraction phenomenon in novel materials could be attributed to oxygen layer faulting, thus, investigation for their structural features well lies in the framework of DIFFaX calculation with our proposed oxygen layer faulting models. 


\section{References}

[1] M.E. Lines, A.M. Glass, Principles and Applications of Ferroelectrics and Related Materials, Clarendon Press, 2001.

[2] R. Comes, M. Lambert, A. Guinier, Solid State Communications, 6 (1968) 715-719.

[3] J.F. Scott, C.A. Paz de Araujo, Science, 246 (1989) 1400-1405.

[4] A.K. Tagantsev, V.O. Sherman, K.F. Astafiev, J. Venkatesh, N. Setter, Journal of Electroceramics, 11 (2003) 5-66.

[5] S.B. Lang, Ferroelectrics, 9 (1975) 141-149.

[6] C.B. Sawyer, Radio Engineers, Proceedings of the IEEE, 19 (1931) 2020-2029.

[7] P. Muralt, Journal of Micromechanics and Microengineering 10 (2000) 136.

[8] S.-E. Park, T.R. Shrout, Ultrasonics, Ferroelectrics, and Frequency Control, IEEE Transactions on, 44 (1997) 1140-1147.

[9] P.M. Moubarak, P. Ben-Tzvi, M.E. Zaghloul, Sensors Journal, IEEE, 12 (2012) 10331042.

[10] T. Fujii, S. Watanabe, M. Suzuki, T. Fujiu, Journal of Vacuum Science \& Technology B, 13 (1995) 1119-1122.

[11] K.K. Shung, J.M. Cannata, Q.F. Zhou, Journal of Electroceramics, 19 (2007) 141-147.

[12] M. Dubois, P. Muralt, Ultrasonics, Ferroelectrics, and Frequency Control, IEEE Transactions on, 45 (1998) 1169-1177.

[13] B. Koc, S. Cagatay, K. Uchino, Ultrasonics, Ferroelectrics, and Frequency Control, IEEE Transactions on, 49 (2002) 495-500.

[14] B. Jaffe, W.R. Cook, H.L. Jaffe, Piezoelectric Ceramics, Academic Press, 1971.

[15] F. Jona, G. Shirane, Ferroelectric Crystals, Pergamon Press, 1962.

[16] J. Curie, P. Curie, Bull. Soc. Min de France, 3 (1880) 12.

[17] G. Busch, P. Scherrer, Ferroelectrics, 71 (1987) 15-16.

[18] B. WUL, Nature, 156 (1945) 1.

[19] L. Cross, R. Newnham, J Am Ceram, 11 (1987) 289-305.

[20] J. Kuwata, K. Uchino, S. Nomura, Ferroelectrics, 37 (1981) 579-582. 
[21] K. Jun, U. Kenji, N. Shoichiro, Japanese Journal of Applied Physics, 21 (1982) 1298.

[22] T.R. Shrout, Z.P. Chang, N. Kim, S. Markgraf, Ferroelectrics Letters Section, 12 (1990) 63-69.

[23] B. Noheda, D.E. Cox, Phase Transitions, 79 (2006) 5-20.

[24] Y.U. Wang, Physical Review B, 74 (2006) 104109.

[25] V.A. Isupov, Ferroelectrics, 266 (2002) 91-102.

[26] T. Takenaka, K. Sakata, Ferroelectrics, 38 (1981) 769-772.

[27] D.G. Schlom, J.H. Haeni, J. Lettieri, C.D. Theis, W. Tian, J.C. Jiang, X.Q. Pan, Materials Science and Engineering: B, 87 (2001) 282-291.

[28] W. Eerenstein, N.D. Mathur, J.F. Scott, Nature, 442 (2006) 759-765.

[29] S.-W. Cheong, M. Mostovoy, Nat Mater, 6 (2007) 13-20.

[30] C.H. Ahn, K.M. Rabe, J.-M. Triscone, Science, 303 (2004) 488-491.

[31] C.D.E. Lakeman, D.A. Payne, Journal of the American Ceramic Society, 75 (1992) 3091-3096.

[32] L.L. Hench, J.K. West, Chemical Reviews, 90 (1990) 33-72.

[33] Y.H. Han, J.B. Appleby, D.M. Smyth, Journal of the American Ceramic Society, 70 (1987) 96-100.

[34] A.J. Bell, Journal of the European Ceramic Society, 28 (2008) 1307-1317.

[35] Y. Saito, H. Takao, T. Tani, T. Nonoyama, K. Takatori, T. Homma, T. Nagaya, M. Nakamura, Nature, 432 (2004) 84-87.

[36] Y. Chang, S.F. Poterala, Z. Yang, S. Trolier-McKinstry, G.L. Messing, Applied Physics Letters, 95 (2009) -.

[37] J.A. Horn, S.C. Zhang, U. Selvaraj, G.L. Messing, S. Trolier-McKinstry, Journal of the American Ceramic Society, 82 (1999) 921-926.

[38] G.L. Messing, S. Trolier-McKinstry, E.M. Sabolsky, C. Duran, S. Kwon, B. Brahmaroutu, P. Park, H. Yilmaz, P.W. Rehrig, K.B. Eitel, E. Suvaci, M. Seabaugh, K.S. Oh, Critical Reviews in Solid State and Materials Sciences, 29 (2004) 45-96.

[39] M. DiDomenico, S.H. Wemple, Physical Review, 155 (1967) 539-545.

[40] E. Soergel, Applied Physics B, 81 (2005) 729-751.

[41] G. Dolino, Applied Physics Letters, 22 (1973) 123-124. 
[42] H. Blank, S. Amelinckx, Applied Physics Letters, 2 (1963) 140-142.

[43] E. Fatuzzo, W.J. Merz, Ferroelectricity, North-Holland Pub. Co., 1967.

[44] G.L. Pearson, W.L. Feldmann, Journal of Physics and Chemistry of Solids, 9 (1959) 28-30.

[45] J. Fousek, M. S̆afránková, J. Kaczér, Applied Physics Letters, 8 (1966) 192-194.

[46] Y. Furuhata, K. Toriyama, Applied Physics Letters, 23 (1973) 361-362.

[47] N. Niizeki, M. Hasegawa, Journal of the Physical Society of Japan, 19 (1964) 550554.

[48] Y.C. Shu, K. Bhattacharya, Philosophical Magazine Part B, 81 (2001) 2021-2054.

[49] W. Heywang, K. Lubitz, W. Wersing, Piezoelectricity: Evolution and Future of a Technology, Springer Berlin Heidelberg, 2008.

[50] G. Arlt, P. Sasko, Journal of Applied Physics, 51 (1980) 4956-4960.

[51] J.J. Brophy, S.L. Webb, Physical Review, 128 (1962) 584-588.

[52] W.P. Mason, H. Jaffe, Proceedings of the IRE, 42 (1954) 921-930.

[53] W. Wersing, G. Zorn, K. Lubitz, J. Mohaupt, Japanese Journal of Applied Physics, 24 (1985) 724.

[54] G. Zorn, W. Wersing, H. Göbel, Japanese Journal of Applied Physics, 24 (1985) 721.

[55] B. Lewis, Proceedings of the Physical Society, 73 (1959) 17.

[56] K.W. Plessner, Proceedings of the Physical Society. Section B, 69 (1956) 1261.

[57] L.A. Shuvalov, Journal of the Physical Society of Japan, 28 (1970) 38-51.

[58] P. Marton, I. Rychetsky, J. Hlinka, Physical Review B, 81 (2010) 144125.

[59] M. Molotskii, Philosophical Magazine Letters, 83 (2003) 763-767.

[60] Y. Su, C.M. Landis, Journal of the Mechanics and Physics of Solids, 55 (2007) 280305.

[61] N. Tsou, J. Huber, in: ASME 2010 Conference on Smart Materials, Adaptive Structures and Intelligent Systems, American Society of Mechanical Engineers, 2010, pp. 285-293.

[62] S.-E. Park, T.R. Shrout, Journal of Applied Physics, 82 (1997) 1804-1811.

[63] M. Yamada, N. Nada, M. Saitoh, K. Watanabe, Applied Physics Letters, 62 (1993) $435-436$. 
[64] J. Webjorn, V. Pruneri, P.S.J. Russell, J.R.M. Barr, D.C. Hanna, Electronics Letters, 30 (1994) 894-895.

[65] W. Satoshi, S. Shingo, N. Tatsuo, S. Takeyuki, O. Minoru, K. Masato, P. Seung-Eek, L.E. Cross, R.S. Thomas, Japanese Journal of Applied Physics, 38 (1999) 5505.

[66] J. Li, D. Liu, Journal of the Mechanics and Physics of Solids, 52 (2004) 1719-1742.

[67] J. Yin, W. Cao, Ferroelectrics, 251 (2001) 93-100.

[68] S.-E. Park, S. Wada, L.E. Cross, T.R. Shrout, Journal of Applied Physics, 86 (1999) 2746-2750.

[69] W. Satoshi, M. Kazuma, K. Hirofumi, T. Takaaki, K. Hirohiko, Japanese Journal of Applied Physics, 43 (2004) 6692.

[70] D. Damjanovic, Journal of the American Ceramic Society, 88 (2005) 2663-2676.

[71] S. Wada, K. Yako, H. Kakemoto, T. Tsurumi, T. Kiguchi, Journal of Applied Physics, 98 (2005) 014109.

[72] W.-F. Rao, Y.U. Wang, Applied Physics Letters, 90 (2007) 041915.

[73] G. Arlt, in: Ultrasonics Symposium, 1990. Proceedings., IEEE 1990, 1990, pp. 733742 vol.732.

[74] G. Arlt, Ferroelectrics, 104 (1990) 217-227.

[75] M.J. Zipparo, K.K. Shung, T.R. Shrout, in: Ultrasonics Symposium, 1995. Proceedings., 1995 IEEE, 1995, pp. 601-604 vol.601.

[76] G. Arlt, Journal of Materials Science, 25 (1990) 2655-2666.

[77] A.G. Luchaninov, A.V. Shil'nikov, L.A. Shuvalov, I.J. Shipkova, Ferroelectrics, 98 (1989) 123-126.

[78] E.K.W. Goo, R.K. Mishra, G. Thomas, Journal of Applied Physics, 52 (1981) 29402943.

[79] P.G. Lucuţa, V. Teodorescu, F. Vasiliu, Applied Physics A, 37 (1985) 237-242.

[80] C. Leslie Eric, Japanese Journal of Applied Physics, 34 (1995) 2525.

[81] D.A. Berlincourt, C. Cmolik, H. Jaffe, Proceedings of the IRE, 48 (1960) 220-229.

[82] W.-F. Rao, Y.U. Wang, Applied Physics Letters, 92 (2008) 102905-102901-102903.

[83] Y. Gang, Y. Zhenxing, Z. Jianqiang, W. Hai, W. Xiaohui, L. Longtu, Journal of Physics D: Applied Physics, 39 (2006) 3702. 
[84] M. Marutake, Journal of the Physical Society of Japan, 11 (1956) 807-814.

[85] A.V. Turik, E.I. Bondarenko, Ferroelectrics, 7 (1974) 303-305.

[86] G. Simpson, Ferroelectrics, 6 (1973) 283-288.

[87] J. Fousek, B. Blurezina, Journal of the Physical Society of Japan, 19 (1964) 830-838.

[88] S.C. Hwang, J.E. Huber, R.M. McMeeking, N.A. Fleck, Journal of Applied Physics, 84 (1998) 1530-1540.

[89] W. Lu, D.N. Fang, C.Q. Li, K.C. Hwang, Acta Materialia, 47 (1999) 2913-2926.

[90] H. Kessler, H. Balke, Journal of the Mechanics and Physics of Solids, 49 (2001) 953978.

[91] J.e. Huber, N.a. Fleck, C.M. Landis, R.M. McMeeking, Journal of the Mechanics and Physics of Solids, 47 (1999) 1663-1697.

[92] J.E. Huber, Current Opinion in Solid State and Materials Science, 9 (2005) 100-106.

[93] J. Rödel, W.S. Kreher, Computational Materials Science, 19 (2000) 123-132.

[94] A. DeSimone, R.D. James, Journal of the Mechanics and Physics of Solids, 50 (2002) 283-320.

[95] J.M. Ball, R.D. James, Archive for Rational Mechanics and Analysis, 100 (1987) 1352.

[96] N.T. Tsou, J.E. Huber, Mechanics of Materials, 42 (2010) 740-753.

[97] J. Rödel, Mechanics of Materials, 39 (2007) 302-325.

[98] F. Li, D. Fang, Mechanics of Materials, 36 (2004) 959-973.

[99] A. Pathak, R.M. McMeeking, Journal of the Mechanics and Physics of Solids, 56 (2008) 663-683.

[100] A. Haug, J.E. Huber, P.R. Onck, E. Van der Giessen, Journal of the Mechanics and Physics of Solids, 55 (2007) 648-665.

[101] P. Potnis, N.-T. Tsou, J. Huber, Materials, 4 (2011) 417-447.

[102] V.Y. Shur, Correlated Nucleation and Self-Organized Kinetics of Ferroelectric Domains, in: Nucleation Theory and Applications, Wiley-VCH Verlag GmbH \& Co. KGaA, 2005, pp. 178-214.

[103] J.E. Zhou, T.-L. Cheng, Y.U. Wang, Journal of Applied Physics, 111 (2012) 024105. 
[104] S. Semenovskaya, A.G. Khachaturyan, Journal of Applied Physics, 83 (1998) 51255136.

[105] V. Ginzburg, L.D. Landau, Sov. Phys. JETP, 20 (1950) 1064.

[106] J.W. Cahn, J.E. Hilliard, The Journal of Chemical Physics, 28 (1958) 258-267.

[107] L.-Q. Chen, Annual Review of Materials Research, 32 (2002) 113-140.

[108] Y. Wang, Journal of Materials Science, 44 (2009) 5225-5234.

[109] A.G. Khachaturyan, Theory of Structural Transformations in Solids, Dover Publications, 2013.

[110] Y.U. Wang, Y.M. Jin, A.G. Khachaturyan, Journal of Applied Physics, 92 (2002) 1351-1360.

[111] E. Fried, M.E. Gurtin, Physica D: Nonlinear Phenomena, 68 (1993) 326-343.

[112] E. Fried, M.E. Gurtin, Physica D: Nonlinear Phenomena, 72 (1994) 287-308.

[113] L.Q. Chen, J. Shen, Computer Physics Communications, 108 (1998) 147-158.

[114] L.D. Landau, E.M. Lifshitz, Statistical Physics, Elsevier Science, 2013.

[115] J.D. Gunton, M. San Miguel, P.S. Sahni, Phase Transitiona and Critical Phemomena vol. 8, Eds. C. Domb and J.L. Lebowitz, Academic Press, (1983) 269-446.

[116] M. Ode, S.G. Kim, T. Suzuki, ISIJ International, 41 (2001) 1076-1082.

[117] D. Raabe, F. Roters, F. Barlat, L.Q. Chen, Continuum Scale Simulation of Engineering Materials: Fundamentals - Microstructures - Process Applications, Wiley, 2006.

[118] L.-Q. Chen, W. Yang, Physical Review B, 50 (1994) 15752-15756.

[119] I.S. Aranson, V.A. Kalatsky, V.M. Vinokur, Physical Review Letters, 85 (2000) 118121.

[120] Y.M. Jin, Y.U. Wang, A.G. Khachaturyan, Applied Physics Letters, 79 (2001) 30713073.

[121] L.-Q. Chen, Journal of the American Ceramic Society, 91 (2008) 1835-1844.

[122] Y.U. Wang, Y.M. Jin, A.M. Cuitiño, A.G. Khachaturyan, Applied Physics Letters, 78 (2001) 2324-2326.

[123] P.K. Panda, Journal of Materials Science, 44 (2009) 5049-5062.

[124] R.E. Cohen, Journal of Physics and Chemistry of Solids, 61 (2000) 139-146. 
[125] Y. Yan, Y.U. Wang, S. Priya, Applied Physics Letters, 100 (2012) 192905.

[126] P. Nørby, S. Johnsen, B.B. Iversen, ACS Nano, 8 (2014) 4295-4303.

[127] S. Grigull, M. Ishimaru, M. Nastasi, C.A. Zorman, M. Mehregany, JCPDSInternational Certre for Diffraction Data 2001, Advanced in X-ray Analysis, 44 (2001) 308-313.

[128] W.-F. Rao, Y.U. Wang, Philosophical Magazine, 90 (2010) 197-217.

[129] C. Daniel, JOM, 60 (2008) 43-48.

[130] M. Armand, J.M. Tarascon, Nature, 451 (2008) 652-657.

[131] J.W. Fergus, Journal of Power Sources, 195 (2010) 939-954.

[132] M.S. Whittingham, Chemical Reviews, 104 (2004) 4271-4302.

[133] E. Antolini, Solid State Ionics, 170 (2004) 159-171.

[134] A. Yamada, S.C. Chung, K. Hinokuma Journal of the Electrochemical Society, 148 (2001) A224-A229.

[135] M.M. Thackeray, A. de Kock, W.I.F. David, Materials Research Bulletin, 28 (1993) 1041-1049.

[136] H. Liu, Y. Wang, K. Wang, Y. Wang, H. Zhou, Journal of Power Sources, 192 (2009) 668-673.

[137] A.K. Padhi, K.S. Nanjundaswamy, J.B. Goodenough, Journal of the Electrochemical Society, 144 (1997) 1188-1194.

[138] A.K. Padhi, K.S. Nanjundaswamy, C. Masquelier, S. Okada, J.B. Goodenough, Journal of the Electrochemical Society, 144 (1997) 1609-1613.

[139] T. Shiratsuchi, S. Okada, T. Doi, J.-i. Yamaki, Electrochimica Acta, 54 (2009) 31453151.

[140] A. Vadivel Murugan, T. Muraliganth, A. Manthiram, Journal of the Electrochemical Society, 156 (2009) A79-A83.

[141] S.G. Stewart, V. Srinivasan, J. Newman, Journal of the Electrochemical Society, 155 (2008) A664-A671.

[142] S. Patoux, L. Daniel, C. Bourbon, H. Lignier, C. Pagano, F. Le Cras, S. Jouanneau, S. Martinet, Journal of Power Sources, 189 (2009) 344-352. 
[143] X.J. Wang, X.Q. Yu, H. Li, X.Q. Yang, J. McBreen, X.J. Huang, Electrochemistry Communications, 10 (2008) 1347-1350.

[144] G. Chen, J.D. Wilcox, T.J. Richardson, Electrochemical and Solid-State Letters, 11 (2008) A190-A194.

[145] T. Ohzuku, A. Ueda, Journal of the Electrochemical Society, 141 (1994) 2972-2977.

[146] J. Zhou, P.H.L. Notten, Journal of Power Sources, 177 (2008) 553-560.

[147] L. Xiao, Y. Yang, Y. Zhao, X. Ai, H. Yang, Y. Cao, J Solid State Electrochem, 12 (2008) 149-153.

[148] M.M. Thackeray, S.-H. Kang, C.S. Johnson, J.T. Vaughey, R. Benedek, S.A. Hackney, Journal of Materials Chemistry, 17 (2007) 3112-3125.

[149] T.A. Arunkumar, E. Alvarez, A. Manthiram, Journal of Materials Chemistry, 18 (2008) 190-198.

[150] N.V. Kosova, E.T. Devyatkina, V.V. Kaichev, Russ J Electrochem, 45 (2009) 277285.

[151] K.-W. Nam, W.-S. Yoon, H. Shin, K.Y. Chung, S. Choi, X.-Q. Yang, Journal of Power Sources, 192 (2009) 652-659.

[152] J. Alper, Science, 296 (2002) 1224-1226.

[153] B.A. Johnson, R.E. White, Journal of Power Sources, 70 (1998) 48-54.

[154] M. Doyle, J. Newman, A.S. Gozdz, C.N. Schmutz, J.M. Tarascon, Journal of the Electrochemical Society, 143 (1996) 1890-1903.

[155] J.R. Dahn, E.W. Fuller, M. Obrovac, U. von Sacken, Solid State Ionics, 69 (1994) 265-270.

[156] D. Aurbach, Y. Ein-Eli, O. Chusid, Y. Carmeli, M. Babai, H. Yamin, Journal of the Electrochemical Society, 141 (1994) 603-611.

[157] E. Peled, D. Golodnttsky, G. Ardel, C. Menachem, D. Bar Tow, V. Eshkenazy, MRS Online Proceedings Library, 393 (1995) 209.

[158] K. Kanamura, S. Toriyama, S. Shiraishi, Z.i. Takehara, Journal of the Electrochemical Society, 142 (1995) 1383-1389.

[159] R. Premanand, A. Durairajan, B. Haran, R. White, B. Popov, Journal of the Electrochemical Society, 149 (2002) A54-A60. 
[160] T. Ohzuku, M. Kitagawa, T. Hirai, Journal of the Electrochemical Society, 137 (1990) 769-775.

[161] N. Imanishi, H. Kashiwagi, T. Ichikawa, Y. Takeda, O. Yamamoto, M. Inagaki, Journal of the Electrochemical Society, 140 (1993) 315-320.

[162] D. Fauteux, R. Koksbang, J Appl Electrochem, 23 (1993) 1-10.

[163] P. Arora, R.E. White, M. Doyle, Journal of the Electrochemical Society, 145 (1998) 3647-3667.

[164] Y.S. Meng, G. Ceder, C.P. Grey, W.-S. Yoon, Y. Shao-Horn, Electrochemical and Solid-State Letters, 7 (2004) A155-A158.

[165] B. Xu, C.R. Fell, M. Chi, Y.S. Meng, Energy \& Environmental Science, 4 (2011) 2223-2233.

[166] P. Barpanda, G. Rousse, T. Ye, C.D. Ling, Z. Mohamed, Y. Klein, A. Yamada, Inorganic Chemistry, 52 (2013) 3334-3341.

[167] B. Ammundsen, J. Paulsen, Advanced Materials, 13 (2001) 943-956.

[168] P. Kalyani, S. Chitra, T. Mohan, S. Gopukumar, Journal of Power Sources, 80 (1999) 103-106.

[169] A.D. Robertson, P.G. Bruce, Chemistry of Materials, 15 (2003) 1984-1992.

[170] G. Yang, H. Ji, P. Gao, A. Hong, H. Ding, S. Roy, J. Pinto, X. Jiang, Journal of the Electrochemical Society, 158 (2011) A1071-A1078.

[171] S. Pierre, L.-A. Bernadette, Journal of Solid State Chemistry, 75 (1988) 90-98.

[172] A. Boulineau, L. Croguennec, C. Delmas, F. Weill, Chemistry of Materials, 21 (2009) 4216-4222.

[173] A. Boulineau, L. Croguennec, C. Delmas, F. Weill, Dalton Transactions, 41 (2012) 1574-1581.

[174] Z.-m. Yu, L.-c. Zhao, Transactions of Nonferrous Metals Society of China, 17 (2007) 659-664.

[175] T. Sebastian, P. Krishna, Random, Non-random, and Periodic Faulting in Crystals, Gordon and Breach Science Publishers, 1994.

[176] B.E. Warren, X-ray Diffraction, Dover Publications, 1969. 
[177] A. Boulineau, L. Croguennec, C. Delmas, F. Weill, Solid State Ionics, 180 (2010) 1652-1659.

[178] M. Tabuchi, A. Nakashima, H. Shigemura, K. Ado, H. Kobayashi, H. Sakaebe, H. Kageyama, T. Nakamura, M. Kohzaki, A. Hirano, R. Kanno, Journal of the Electrochemical Society, 149 (2002) A509-A524.

[179] X.C. Zhang Lianqi, Yang Ruijuan, Progress in Chemistry, 23 (2011) 410-417.

[180] K. Numata, C. Sakaki, S. Yamanaka, Chemistry letters, (1997) 725-726.

[181] K. Numata, C. Sakaki, S. Yamanaka, Solid State Ionics, 117 (1999) 257-263.

[182] H. Shigemura, M. Tabuchi, H. Sakaebe, H. Kobayashi, H. Kageyama, Journal of the Electrochemical Society, 150 (2003) A638-A644.

[183] Y. Koyama, H. Arai, I. Tanaka, Y. Uchimoto, Z. Ogumi, Chemistry of Materials, 24 (2012) 3886-3894.

[184] Y. Mo, S.P. Ong, G. Ceder, Physical Review B, 84 (2011) 205446.

[185] A.J.C. Wilson, Imperfections in the Structure of Cobalt. II. Mathematical Treatment of Proposed Structure, 1942.

[186] M.S. Paterson, Journal of Applied Physics, 23 (1952) 805-811.

[187] T.R. Welberry, Reports on Progress in Physics, 48 (1985) 1543.

[188] M.M.J. Treacy, J.M. Newsam, M.W. Deem, Proceedings of the Royal Society of London. Series A: Mathematical and Physical Sciences, 433 (1991) 499-520.

[189] M. Casas-Cabanas, J. Rodríguez-Carvajal, M. Palacín, Zeitschrift für Kristallographie, Suppl, 23 (2006) 243-248.

[190] A.F. Devonshire, The London, Edinburgh, and Dublin Philosophical Magazine and Journal of Science, 40 (1949) 1040-1063.

[191] W. Yang, L.-Q. Chen, Journal of the American Ceramic Society, 78 (1995) 25542556.

[192] H.-L. Hu, L.-Q. Chen, Materials Science and Engineering: A, 238 (1997) 182-191.

[193] H.-L. Hu, L.-Q. Chen, Journal of the American Ceramic Society, 81 (1998) 492-500.

[194] A.J. Bell, Journal of Applied Physics, 89 (2001) 3907-3914.

[195] A. Amin, M.J. Haun, B. Badger, H. McKinstry, L.E. Cross, Ferroelectrics, 65 (1985) 107-130. 
[196] F.K. Lotgering, Journal of Inorganic and Nuclear Chemistry, 9 (1959) 113-123.

[197] B.D. Cullity, S.R. Stock, Elements of X-ray Diffraction, Prentice Hall, 2001.

[198] M.M.J. Treacy, J.M. Newsam, M.W. Deem, DIFFaX Manual, (2005) Software (v1.813) downloaded through: http://www.public.asu.edu/ mtreacy/DIFFaX.html

[199] K. Momma, F. Izumi, Journal of Applied Crystallography, 44 (2011) 1272-1276.

[200] T. Ikeda, Fundamentals of Piezoelectricity, Oxford University Press, 1996.

[201] K. Uchino, Ferroelectric Devices, Taylor \& Francis, 2000.

[202] W.-F. Rao, Y.U. Wang, Applied Physics Letters, 90 (2007) 182906.

[203] W.-F. Rao, T.-L. Cheng, Y.U. Wang, Applied Physics Letters, 96 (2010) 122903.

[204] Y.M. Jin, Y.U. Wang, A.G. Khachaturyan, J.F. Li, D. Viehland, Physical Review Letters, 91 (2003) 197601.

[205] Y.U. Wang, Physical Review B, 73 (2006) 014113.

[206] Y.U. Wang, Physical Review B, 76 (2007) 024108.

[207] J.W. Gibbs, The collected works of J. Willard Gibbs, Yale University Press, 1948.

[208] J.C. Maxwell, A Treatise on Electricity and Magnetism, Cambridge University Press, 2010.

[209] J.D. Eshelby, The Determination of the Elastic Field of an Ellipsoidal Inclusion, and Related Problems, 1957.

[210] U.F. Kocks, C.N. Tomé, H.R. Wenk, Texture and Anisotropy: Preferred Orientations in Polycrystals and Their Effect on Materials Properties, Cambridge University Press, 2000.

[211] J.E. Zhou, Y.U. Wang, Computational Study of Texture Development During Templated Grain Growth, in: EPD Congress 2014, John Wiley \& Sons, Inc., 2014, pp. 299-307.

[212] Y. Yan, K.-H. Cho, S. Priya, Applied Physics Letters, 100 (2012) 132908.

[213] Y.U. Wang, Applied Physics Letters, 96 (2010) 232901.

[214] M. Wang, N. Pan, Materials Science and Engineering: R: Reports, 63 (2008) 1-30.

[215] D. Mohanty, A.S. Sefat, J. Li, R.A. Meisner, A.J. Rondinone, E.A. Payzant, D.P. Abraham, D.L. Wood Iii, C. Daniel, Physical Chemistry Chemical Physics, 15 (2013) 19496-19509. 
[216] A.C. Larson, R.B.V. Dreele, Los Alamos National Laboratory Report LAUR, (1994) 86-748.

[217] J. Hlinka, P. Márton, Physical Review B, 74 (2006) 104104.

[218] M. Taherinejad, D. Vanderbilt, P. Marton, V. Stepkova, J. Hlinka, Physical Review B, 86 (2012) 155138.

[219] Y. Gu, Z. Hong, J. Britson, L.-Q. Chen, Applied Physics Letters, 106 (2015) 022904.

[220] C. Kittel, Introduction to Solid State Physics, Wiley, 2004.

[221] B.H. Toby, J. Appl. Cryst. , 34 (2001) 210-213.

[222] J.A. Kaduk, J. Reid, Powder Diffraction, 26 (2011) 88-93.

[223] S. Kirkpatrick, C.D. Gelatt, M.P. Vecchi, Science, 220 (1983) 671-680. 


\section{Appendix A: Energy Barrier to Ferroelectric Nucleation Due to Electrostatic and Elastostatic Interactions}

To illustrate the close relevance between the analytical solution of special case of spherical nucleus and computer simulation of general case of domain evolution, it is worthy to derive the electrostatic and elastostatic interaction energies of spherical nucleus from the same general formula given in Eq. (3.4) as used in the phase field modeling. The results thus obtained are identical to the classical solutions of electrostatic and elastostatic inclusion problems $[\underline{109}, \underline{208}, \underline{209]}$.

Consider a spherical particle (nucleus) of radius $R$ as described by the following shape function:

$\rho(\mathbf{r})=\rho(r)=\left\{\begin{array}{lll}1 & \text { if } & r \leq R \\ 0 & \text { if } & r>R\end{array}\right.$,

whose Fourier transform is:

$\tilde{\rho}(\mathbf{k})=\tilde{\rho}(k)=\int \rho(r) e^{-i \mathbf{k} \cdot \mathbf{r}} d^{3} r=3 V \frac{\sin k R-k R \cos k R}{(k R)^{3}}$,

where $V=4 \pi R^{3} / 3$ is the particle volume. Using Parseval's theorem and the unit value of $\rho(\mathbf{r})$ within the particle, Eqs. (A1) and (A2) give:

$V=\int|\rho(\mathbf{r})|^{2} d^{3} r=\int \frac{d^{3} k}{(2 \pi)^{3}}|\tilde{\rho}(\mathbf{k})|^{2}=\int \frac{k^{2} \sin \theta d \theta d \varphi d k}{(2 \pi)^{3}}\left|3 V \frac{\sin k R-k R \cos k R}{(k R)^{3}}\right|^{2}$

\# The contents in Appendix A were previously published in J. Appl. Phys., 111, 024105-1-13, 2012. Refer Appendix D for granted permission to be republished. 
where $\theta$ and $\varphi$ are the zenith (polar) and azimuth angles, respectively, of spherical coordinate system. The double spherical angle integral gives solid angle $4 \pi$, i.e., $\int_{0}^{\pi} \sin \theta d \theta \int_{0}^{2 \pi} d \varphi=4 \pi$, thus it is obtained from Eq. (A3):

$\int_{0}^{\infty} \frac{k^{2} d k}{(2 \pi)^{3}}\left|3 \frac{\sin k R-k R \cos k R}{(k R)^{3}}\right|^{2}=\frac{1}{4 \pi V}$

Eq. (A4) will be used in the following to evaluate the k-space integral in Eq. (3.4) to obtain electrostatic and elastostatic energies.

The electrostatic energy of an arbitrary distribution of polarization $\mathbf{P}(\mathbf{r})$, as from Eq. (3.4), is:

$$
E^{\text {electr }}=\frac{1}{2 \varepsilon_{0}} \int \frac{d^{3} k}{(2 \pi)^{3}}|\mathbf{n} \cdot \tilde{\mathbf{P}}(\mathbf{k})|^{2} .
$$

For a spherical particle of homogeneous polarization, $\mathbf{P}(\mathbf{r})$ can be written as:

$\mathbf{P}(\mathbf{r})=\mathbf{P} \rho(\mathbf{r})$.

Substituting Eqs. (A2) and (A6) into Eq. (A5) and changing to spherical coordinate system give:

$$
E^{\text {electr }}=\frac{V^{2}}{2 \varepsilon_{0}} \int_{0}^{\pi} \int_{0}^{2 \pi} \sin \theta d \theta d \varphi|\mathbf{n} \cdot \mathbf{P}|^{2} \int_{0}^{\infty} \frac{k^{2} d k}{(2 \pi)^{3}}\left|3 \frac{\sin k R-k R \cos k R}{(k R)^{3}}\right|^{2} .
$$

Using Eq. (A4) into Eq. (A7) gives:

$$
E^{\text {electr }}=\frac{P^{2}}{2 \varepsilon_{0}} V\left\langle|\mathbf{n} \cdot \mathbf{p}|^{2}\right\rangle,
$$

where $\mathbf{p}=\mathbf{P} / P$ is a unit vector along polarization direction, and 
$\left\langle|\mathbf{n} \cdot \mathbf{p}|^{2}\right\rangle=\frac{1}{4 \pi} \int_{0}^{\pi} \int_{0}^{2 \pi} \sin \theta d \theta d \varphi|\mathbf{n} \cdot \mathbf{p}|^{2}$

which is the directional average of $|\mathbf{n} \cdot \mathbf{p}|^{2}$ over the entire $4 \pi$ solid angle. For convenience and without loss of generality, consider $\mathbf{P}$ along zenith direction (polar axis), thus $\mathbf{n} \cdot \mathbf{p}=\cos \theta$, and Eq. (A9) gives:

$\left\langle|\mathbf{n} \cdot \mathbf{p}|^{2}\right\rangle=\frac{1}{3}$

It is worth noting that Eq. (A10) gives the depolarization factor of a sphere [220]. Therefore, the electrostatic energy of a spherical homogeneous ferroelectric nucleus is:

$E^{\text {electr }}=\frac{P^{2}}{6 \varepsilon_{0}} V$,

or, equivalently, the electrostatic energy density of the nucleus is:

$e^{\text {electr }}=\frac{P^{2}}{6 \varepsilon_{0}}$.

The elastostatic energy of an arbitrary distribution of electrostrictive strain $\varepsilon_{i j}(\mathbf{r})$, as from Eq. (3.4), is [109]:

$E^{\text {elast }}=\frac{1}{2} \int \frac{d^{3} k}{(2 \pi)^{3}} K_{i j k l} \tilde{\varepsilon}_{i j} \tilde{\varepsilon}_{k l}^{*}$.

As shown by Khachaturyan [109], the classical Eshelby inclusion problem [209] is a special case of Eq. (A13). For a spherical particle of homogeneous polarization as described by Eq. (A6), the electrostrictive strain is also homogeneous:

$\varepsilon_{i j}(\mathbf{r})=\varepsilon_{i j} \rho(\mathbf{r})$, 
where $\varepsilon_{i j}$ is given by Eq. (5). Substituting Eqs. (A2) and (A14) into Eq. (A13) and changing to spherical coordinate system give:

$$
E^{\text {elast }}=\frac{V^{2}}{2} \int_{0}^{\pi} \int_{0}^{2 \pi} \sin \theta d \theta d \varphi K_{i j k l} \varepsilon_{i j} \varepsilon_{k l} \int_{0}^{\infty} \frac{k^{2} d k}{(2 \pi)^{3}}\left|3 \frac{\sin k R-k R \cos k R}{(k R)^{3}}\right|^{2} .
$$

Using Eq. (A4) into Eq. (A15) gives:

$$
E^{\mathrm{elast}}=\frac{V}{2}\left\langle K_{i j k l} \varepsilon_{i j} \varepsilon_{k l}\right\rangle
$$

where

$$
\left\langle K_{i j k l} \varepsilon_{i j} \varepsilon_{k l}\right\rangle=\frac{1}{4 \pi} \int_{0}^{\pi} \int_{0}^{2 \pi} \sin \theta d \theta d \varphi K_{i j k l} \varepsilon_{i j} \varepsilon_{k l}
$$

In order to analytically evaluate above directional average, isotropic elasticity is considered, where the elastic modulus tensor and Green function tensor are expressed explicitly in terms of Young's modulus $E$ and Poisson's ratio $v$ as:

$$
\begin{aligned}
& C_{i j k l}=\frac{E v}{(1+v)(1-2 v)} \delta_{i j} \delta_{k l}+\frac{E}{2(1+v)}\left(\delta_{i k} \delta_{j l}+\delta_{i l} \delta_{j k}\right), \\
& \Omega_{i k}=\frac{2(1+v)}{E} \delta_{i k}-\frac{1+v}{E(1-v)} n_{i} n_{k},
\end{aligned}
$$

where $\delta_{i j}$ is Kronecker delta. Substituting Eqs. (A18) and (A19) into $K_{i j k l}=C_{i j k l}-n_{p} C_{i j p q} \Omega_{q r} C_{k l r s} n_{s}$ gives:

$$
\begin{aligned}
K_{i j k l}= & \frac{E v}{1-v^{2}}\left(\delta_{i j} \delta_{k l}-\delta_{i j} n_{k} n_{l}-\delta_{k l} n_{i} n_{j}\right) \\
& +\frac{E}{2(1+v)}\left(\delta_{i k} \delta_{j l}+\delta_{i l} \delta_{j k}-\delta_{i k} n_{j} n_{l}-\delta_{i l} n_{j} n_{k}-\delta_{j k} n_{i} n_{l}-\delta_{j l} n_{i} n_{k}\right)+\frac{E}{1-v^{2}} n_{i} n_{j} n_{k} n_{l}
\end{aligned}
$$

Using Eq. (A20) into Eq. (A17) gives: 


$$
\begin{aligned}
\left\langle K_{i j k l} \varepsilon_{i j} \varepsilon_{k l}\right\rangle= & \frac{E}{1-v^{2}}\left[v\left(\varepsilon_{k k}\right)^{2}+(1-v) \varepsilon_{i j} \varepsilon_{i j}\right. \\
& \left.-2 v \varepsilon_{k k}\left\langle\varepsilon_{i j} n_{i} n_{j}\right\rangle-2(1-v)\left\langle\varepsilon_{k i} \varepsilon_{k j} n_{i} n_{j}\right\rangle+\left\langle\left(\varepsilon_{i j} n_{i} n_{j}\right)^{2}\right\rangle\right]
\end{aligned}
$$

To be specific, the following electrostrictive strain of tetragonal symmetry is considered:

$$
\boldsymbol{\varepsilon}=\left[\begin{array}{ccc}
\varepsilon_{1} & 0 & 0 \\
0 & \varepsilon_{2} & 0 \\
0 & 0 & \varepsilon_{2}
\end{array}\right] .
$$

Substitution of Eq. (A22) into Eq. (A21) yields:

$$
\left\langle K_{i j k l} \varepsilon_{i j} \varepsilon_{k l}\right\rangle=\frac{E}{1-v^{2}}\left[\varepsilon_{1}^{2}+\varepsilon_{2}^{2}+2 v \varepsilon_{1} \varepsilon_{2}-2\left(\varepsilon_{1}-\varepsilon_{2}\right)\left(\varepsilon_{1}+v \varepsilon_{2}\right)\left\langle n_{1}^{2}\right\rangle+\left(\varepsilon_{1}-\varepsilon_{2}\right)^{2}\left\langle n_{1}^{4}\right\rangle\right] .
$$

For convenience, choose the tetragonal axis as the zenith direction (polar axis), thus $n_{1}=\cos \theta$, and $\left\langle n_{1}^{2}\right\rangle=\frac{1}{3},\left\langle n_{1}^{4}\right\rangle=\frac{1}{5}$

Using Eqs. (A23) and (A24) into Eq. (A16) yields the elastostatic energy of a spherical homogeneous ferroelectric nucleus:

$$
E^{\text {elast }}=\frac{E}{15\left(1-v^{2}\right)}\left[4 \varepsilon_{1}^{2}+(9+5 v) \varepsilon_{2}^{2}+2(1+5 v) \varepsilon_{1} \varepsilon_{2}\right] V,
$$

or, equivalently, the elastostatic energy density of the nucleus is:

$$
e^{\text {elast }}=\frac{E}{15\left(1-v^{2}\right)}\left[4 \varepsilon_{1}^{2}+(9+5 v) \varepsilon_{2}^{2}+2(1+5 v) \varepsilon_{1} \varepsilon_{2}\right] .
$$

Eqs. (A25) and (A26) are identical to the results obtained from Eshelby's method [209]. 
For polarization along [100] axis, such as in a nucleus of tetragonal ferroelectric phase, the electrostrictive strain components are:

$\varepsilon_{1}=Q_{11} P^{2}, \varepsilon_{2}=Q_{12} P^{2}$,

where $Q_{11}=Q_{1111}$ and $Q_{12}=Q_{1122}$ are the electrostrictive constants (i.e., the tensor components in Voigt matrix notation). Substitution of Eq. (A27) into Eq. (A26) gives:

$e^{\text {elast }}=\frac{E P^{4}}{15\left(1-v^{2}\right)}\left[4 Q_{11}^{2}+(9+5 v) Q_{12}^{2}+2(1+5 v) Q_{11} Q_{12}\right]$

It is worth noting that, due to elastic isotropy, Eq. (A26) also applies to nucleus of rhombohedral ferroelectric phase with polarization along [111] axis, where the electrostrictive strain is:

$\boldsymbol{\varepsilon}=\left[\begin{array}{lll}\alpha & \beta & \beta \\ \beta & \alpha & \beta \\ \beta & \beta & \alpha\end{array}\right]$

where

$\alpha=\frac{1}{3}\left(Q_{11}+2 Q_{12}\right) P^{2}, \beta=\frac{1}{6} Q_{44} P^{2}$,

where $Q_{44}=4 Q_{1212}$. Changing to a coordinate system with $x$-axis along [111] direction, the strain tensor in Eq. (A29) is transformed into the same form as in Eq. (A22), with the following principal strain values:

$\varepsilon_{1}=\alpha+2 \beta=\frac{1}{3}\left(Q_{11}+2 Q_{12}+Q_{44}\right) P^{2}, \varepsilon_{2}=\alpha-\beta=\frac{1}{6}\left(2 Q_{11}+4 Q_{12}-Q_{44}\right) P^{2}$.

Substitution of Eq. (A31) into Eq. (A26) gives:

$e^{\text {elast }}=\frac{E P^{4}}{180\left(1-v^{2}\right)}\left[20(1+v) Q_{11}^{2}+80(1+v) Q_{12}^{2}+(7-5 v) Q_{44}^{2}+80(1+v) Q_{11} Q_{12}\right]$ 


\section{Appendix B: DIFFaX Resolution and Broadening Parameters}

\section{B1. Increasing Resolution for Spectrum Calculation by Using DIFFaX}

Source codes and compiled executable file are supplied online for non-commercial software DIFFaX (v1.813) [198] under windows operation system. One issue related to the compiled executable file is that the resolution for spectrum calculation is constrained by default parameter, which is to say that the maximum reliable resolution is 0.002 (two theta step). One way to increase resolution is to modify source code and re-compile. A parameter named "MAX_SP" located in the file "DIFFaX.par" could be changed to a larger number according to your required resolution. In order to accomplish higher resolution calculation, for us, we change MAX_SP value from 20001 to 80001 and recompile the source codes again.

\section{B2. Instrumental Broadening Parameter Conventions in DIFFaX and GSAS}

The three instrumental Gaussian broadening parameters denoted by u, v, w and GU, GV, GW in DIFFaX manual [198] and in GSAS software interface [216, 221] respectively are interchangeable through multiplying a constant value.

In GSAS, variance of the Gaussian profile function is written as the form [222]

$\sigma^{2}=U \cdot \tan ^{2} \theta+V \cdot \tan \theta+W$, while in DIFFaX manual [198], the form to represent full- 
width half-maximum is $\Gamma^{2}=u \tan ^{2} \theta+v \tan \theta+w$. Due to the difference in the form definition of Gaussian broadening function and angle unit between GSAS and DIFFaX (for GSAS, the angle unit is centidegree), the two quantities have the relation $\sigma^{2}=\frac{(100)^{2}}{8 \ln (2)} \Gamma^{2}=1803.4 \Gamma^{2}$. To obtain the same Gaussian profile function, the parameters under different software environments should follow the interchange relations $U=1803.4 u, V=1803.4 v, W=1803.4 w$ in order to recover each other. 


\section{Appendix C: Simulated Annealing Algorithm}

\section{C1. Difference between Two Conventional Residual Error Analysis Forms}

$$
\begin{aligned}
& R_{p}=\frac{\sum\left|I_{c a l}-I_{\text {exp }}\right|}{\sum\left|I_{\text {exp }}\right|} \\
& R_{w}=\frac{\sum\left(I_{c a l}-I_{\text {exp }}\right)^{2}}{\sum\left(I_{\text {exp }}\right)^{2}}
\end{aligned}
$$

Conventionally, there are two ways to define residual error for x-ray diffraction pattern as shown in (C1). First, algorithm named least square is adopted to minimize the objective function $F=\sum\left(I_{c a l}-I_{\text {exp }}\right)^{2}$. Experiment X-ray diffraction data $\left(30 \% \mathrm{LiCoO}_{2}\right)$ are used as objective curve to be fitted. Databases constructed from DIFFaX [188] contain eleven $\mathrm{x}$-ray diffraction patterns with stacking fault from $0 \%$ to $100 \%$ under intergrowth model framework and one curve with GSAS fitting parameters under solid solution model (solid solution is irrelevant to stacking fault, which is thus represented as a dotted horizontal line in figures). It is worth to mention that stacking fault here represents the percentage of existence of perfect stacking vector, which is to say that $100 \%$ stacking fault shown on the $\mathrm{x}$-axis means $100 \%$ perfect stacking. Fig. $\mathrm{C} 1.1$ presents $\mathrm{R}_{\mathrm{w}}$ and $\mathrm{R}_{\mathrm{p}}$ values by fitting experimental data with each individual curve from DIFFaX calculated databases with different fitting range. This fitting procedure is mainly to adjust peak height scale and linear background for each intergrowth case with specific stacking fault probability and solid solution case in order to get the best fit with experiment data individually. Based on 
the fitting results shown on Fig. C1.1, intergrowth notation is superior to solid solution upon fitting with experimental data except for $100 \%$ perfect stacking which contributes quite sharp intensities if $R_{p}$ value is adopted in all fitting range. When the selected fitting range is gradually decreased as shown in Figs. C1.1(a)-(c), with $R_{w}$ form, intergrowth case tends to perform better than solid solution case in a much narrow fitting range among which stacking fault feature dominates. Such behavior shows us that $R_{w}$ form could conceal the localized feature (usually centralized around certain angle ranges) of stacking faults on the X-ray diffraction pattern by involving extra range of large Bragg peak intensities. It is worth to note that $R_{p}$ form is non-sensitive to range selection which reinforces its reliability.

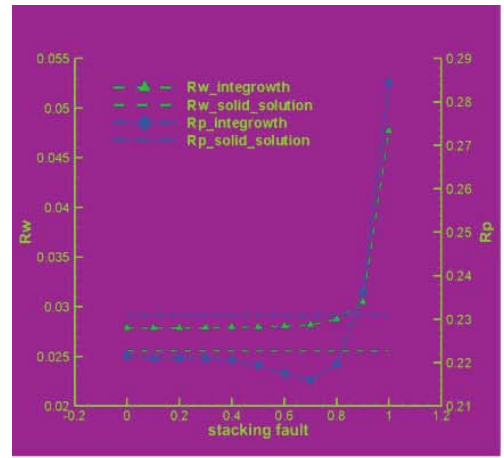

(a)

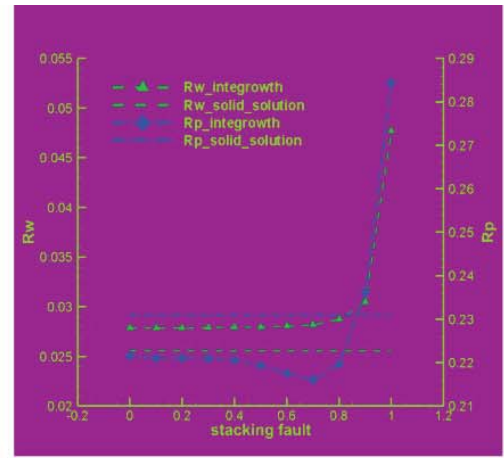

(b)

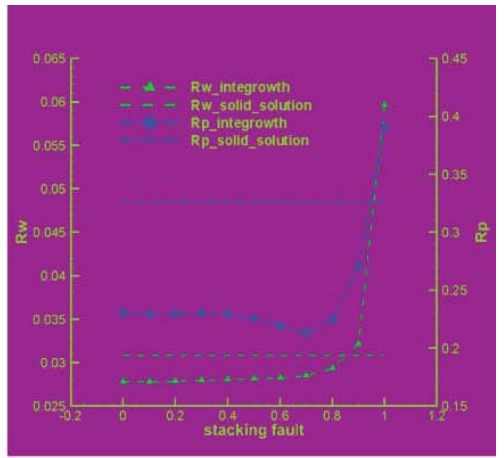

(c)

Figure C1.1 $R_{w}$ and $R_{p}$ are different residual error forms defined in (C1). Green dashed lines with symbol and without symbol represent $\mathrm{R}_{\mathrm{w}}$ values for intergrowth model and solid solution model respectively. Blue dashed lines with symbol and without symbol represent $R_{p}$ values under intergrowth model and solid solution model respectively. The fitting range (20) is (a) 3.002 to 29.0 ; (b) 3.002 to 20.0 ; (c) 3.002 to 9.5 .

A qualitative math analysis is attempted to give some hints on the different behaviors by using either $R_{w}$ or $R_{p}$. We could rewrite (C1) as new forms: 


$$
\begin{aligned}
& R_{p}=\frac{\left|\Delta I_{1}\right|+\left|\Delta I_{2}\right|+\left|\Delta I_{3}\right|+\ldots}{I_{\exp 1}+I_{\exp 2}+I_{\exp 3}+\ldots} \\
& R_{w}=\frac{\Delta I_{1}^{2}+\Delta I_{2}^{2}+\Delta I_{3}^{2}+\ldots}{I_{\exp 1}^{2}+I_{\exp 2}^{2}+I_{\exp 3}^{2}+\ldots}
\end{aligned}
$$

where $\Delta I_{1}$ is abbreviated for $\Delta I\left(\theta=\theta_{1}\right), I_{\text {exp } 1}$ is abbreviated for $I_{\exp }\left(\theta=\theta_{1}\right)$. We want to discuss on the different behaviors the two forms will present when we apply them to the same fitting problem. Eqs. (C3) define the discretized form of $R_{p}$ value $R_{p}(n-1)$ for $n$-1 data points and $R_{p}(n)$ for $n$ data points with one more data involved in. $\frac{\Delta R_{p}}{R_{p}(n-1)}$ describes the relative $R_{p}$ value change if one more data point is involved. Eqs. (C4) define the discretized form of $R_{w}$ value $R_{w}(n-1)$ for $n$ - 1 data points and $R_{w}(n)$ for $n$ data points with one more data involved in. $\frac{\Delta R_{w}}{R_{w}(n-1)}$ describes the relative $R_{w}$ value change if one more data point is involved. Eq. (C5) calculates the ratio between relative $R_{p}$ value change and relative $R_{w}$ value change if one more data point is involved. (C6) and (C7) show such ratio is reasonably smaller than 1 , which means $R_{p}$ value increases more slowly as compared with $R_{w}$ value when the fitting range broadens. Thus, we could denote $\frac{\Delta R_{p}}{R_{p}(n-1)}: \frac{\Delta R_{w}}{R_{w}(n-1)}$ as characteristic factor to describe the relative developing speed for $R_{p}$ and $R_{w}$. Although each of the ways to describe the residual error has its own advantages (Online Contents: http://reference.iucr.org/dictionary/R_factor), $R_{p}$ value is therefore the better object function to perform minimization algorithm since our purpose is to extract stacking fault information from data. 


$$
\begin{aligned}
& R_{p}(n-1)=\frac{\sum_{i=1}^{n-1}\left|\Delta I_{i}\right|}{\sum_{i=1}^{n-1} I_{\exp i}} \quad R_{p}(n)=\frac{\sum_{i=1}^{n-1}\left|\Delta I_{i}\right|+\left|\Delta I_{n}\right|}{\sum_{i=1}^{n-1} I_{\exp i}+I_{\exp n}} \\
& \frac{\Delta R_{p}}{R_{p}(n-1)}=\frac{\frac{\sum_{i=1}^{n-1}\left|\Delta I_{i}\right|+\left|\Delta I_{n}\right|}{\sum_{i=1}^{n-1} I_{\exp i}+I_{\exp n}}-\frac{\sum_{i=1}^{n-1}\left|\Delta I_{i}\right|}{\sum_{i=1}^{n-1} I_{\exp i}\left|\Delta I_{i}\right|}}{\frac{\sum_{i=1}^{n-1} I_{\exp i}}{\sum_{i=1}^{n-1}}}=\frac{\left|\Delta I_{n}\right| \sum_{i=1}^{n-1} I_{\exp i}-I_{\exp n} \sum_{i=1}^{n-1}\left|\Delta I_{i}\right|}{\left(\sum_{i=1}^{n-1} I_{\exp i}+I_{\exp n}\right) \sum_{i=1}^{n-1}\left|\Delta I_{i}\right|}
\end{aligned}
$$

$$
\begin{aligned}
& R_{w}(n-1)=\frac{\sum_{i=1}^{n-1}\left(\Delta I_{i}\right)^{2}}{\sum_{i=1}^{n-1} I_{\exp i}^{2}} \quad R_{w}(n)=\frac{\sum_{i=1}^{n-1}\left(\Delta I_{i}\right)^{2}+\left(\Delta I_{n}\right)^{2}}{\sum_{i=1}^{n-1} I_{\exp i}{ }^{2}+I_{\exp n}^{2}} \\
& \frac{\Delta R_{w}}{R_{w}(n-1)}=\frac{\frac{\sum_{i=1}^{n-1}\left(\Delta I_{i}\right)^{2}+\left(\Delta I_{n}\right)^{2}}{\sum_{i=1}^{n-1} I_{\exp i}{ }^{2}+I_{\exp n}{ }^{2}}-\frac{\sum_{i=1}^{n-1}\left(\Delta I_{i}\right)^{2}}{\sum_{i=1}^{n-1} I_{\exp i}{ }^{2}}}{\sum_{i=1}^{n-1}\left(\Delta I_{i}\right)^{2}}=\frac{\left(\Delta I_{n}\right)^{2} \sum_{i=1}^{n-1} I_{\exp i}{ }^{2}-I_{\exp n}{ }^{2} \sum_{i=1}^{n-1}\left(\Delta I_{i}\right)^{2}}{\left(\sum_{i=1}^{n-1} I_{\exp i}{ }^{2}+I_{\exp n}{ }^{2}\right) \sum_{i=1}^{n-1}\left(\Delta I_{i}\right)^{2}} \\
& \frac{\Delta R_{p}}{R_{p}(n-1)}: \frac{\Delta R_{w}}{R_{w}(n-1)}=\frac{\left|\Delta I_{n}\right| \sum_{i=1}^{n-1} I_{\exp i}-I_{\exp n} \sum_{i=1}^{n-1}\left|\Delta I_{i}\right|}{\left(\sum_{i=1}^{n-1} I_{\exp i}+I_{\exp n}\right) \sum_{i=1}^{n-1}\left|\Delta I_{i}\right|}: \frac{\left(\Delta I_{n}\right)^{2} \sum_{i=1}^{n-1} I_{\exp i}{ }^{2}-I_{\exp n}{ }^{2} \sum_{i=1}^{n-1}\left(\Delta I_{i}\right)^{2}}{\left(\sum_{i=1}^{n-1} I_{\exp i}{ }^{2}+I_{\exp n}{ }^{2}\right) \sum_{i=1}^{n-1}\left(\Delta I_{i}\right)^{2}} \\
& =\frac{\left|\Delta I_{n}\right| \sum_{i=1}^{n-1} I_{\exp i}-I_{\exp n} \sum_{i=1}^{n-1}\left|\Delta I_{i}\right|}{\left(\sum_{i=1}^{n-1} I_{\exp i}+I_{\exp n}\right) \sum_{i=1}^{n-1}\left|\Delta I_{i}\right|} \frac{\left(\sum_{i=1}^{n-1} I_{\exp i}{ }^{2}+I_{\exp n}{ }^{2}\right) \sum_{i=1}^{n-1}\left(\Delta I_{i}\right)^{2}}{\left(\Delta I_{n}\right)^{2} \sum_{i=1}^{n-1} I_{\exp i}{ }^{2}-I_{\exp n}{ }^{2} \sum_{i=1}^{n-1}\left(\Delta I_{i}\right)^{2}} \\
& =\frac{\left|\Delta I_{n}\right| \sum_{i=1}^{n} I_{\exp i}-I_{\exp n} \sum_{i=1}^{n}\left|\Delta I_{i}\right|}{\left(\sum_{i=1}^{n} I_{\exp i}\right) \sum_{i=1}^{n-1}\left|\Delta I_{i}\right|} \frac{\left(\sum_{i=1}^{n} I_{\exp i}{ }^{2}\right) \sum_{i=1}^{n-1}\left(\Delta I_{i}\right)^{2}}{\left(\Delta I_{n}\right)^{2} \sum_{i=1}^{n} I_{\exp i}{ }^{2}-I_{\exp n}{ }^{2} \sum_{i=1}^{n}\left(\Delta I_{i}\right)^{2}}
\end{aligned}
$$




$$
\begin{aligned}
& \frac{\Delta R_{p}}{R_{p}(n-1)}: \frac{\Delta R_{w}}{R_{w}(n-1)} \sim \frac{1}{\left|\Delta I_{n}\right|} \frac{\sum_{i=1}^{n-1}\left(\Delta I_{i}\right)^{2}}{\sum_{i=1}^{n-1}\left|\Delta I_{i}\right|} \text { if } n \uparrow \uparrow \uparrow \\
& \text { if }\left(\sum_{i=1}^{n-1}\left(\Delta I_{i}\right)^{2}-\left|\Delta I_{n}\right| \sum_{i=1}^{n-1}\left|\Delta I_{i}\right|\right)=\sum_{i=1}^{n-1}\left|\Delta I_{i}\right|\left(\left|\Delta I_{i}\right|-\left|\Delta I_{n}\right|\right)<0 \\
& \frac{\Delta R_{p}}{R_{p}(n-1)}: \frac{\Delta R_{w}}{R_{w}(n-1)} \sim \frac{1}{\left|\Delta I_{n}\right|} \frac{\sum_{i=1}^{n-1}\left(\Delta I_{i}\right)^{2}}{\sum_{i=1}^{n-1}\left|\Delta I_{i}\right|}<1
\end{aligned}
$$

\section{C2. Searching Database with Simulated Annealing Algorithm [223]}

Stacking fault related broaden peaks are our main concern and usually contribute much lower intensities compared with neighborhood Bragg peaks. Using $R_{w}$ to construct minimization function to do least square refinement has the potential risk to omit the contribution coming from stacking fault as stated previously. Therefore, we choose $R_{p}$ notation to construct our optimization function. In order to perform minimization search, least square method first comes to our mind due to its simplicity and wide use. However, least square method has the high requirement for smoothness of optimization functions, thus, applying $R_{p}$ to be optimization function involves in intrinsic difficulty to perform least square searching algorithm. Simulated annealing algorithm for non-linear optimization problem is competent for such issue because it does not have any constrain on the form of object functions.

Formula (1-x) $\mathrm{Li}_{2} \mathrm{MnO}_{3} \cdot x \mathrm{LiCoO}_{2}$ is adopted to label different sample compositions. Table C2.1 summarizes sample numbers and their corresponding composition $x$. For each 
sample, we use solid solution model to fit X-ray diffraction data with profile parameters GU, GV, GW, lattice parameters, background (number 7 linear, 2 terms), thermal factor $\left(\mathrm{U}_{0}\right)$, coordination $\left(\mathrm{X}_{0}\right)$, and occupation fraction $\left(\mathrm{F}_{0}\right)$ using GSAS software. Then, databases with each composition are constructed with DIFFaX software based on the fitting parameters from GSAS. Solid solution model is constructed exactly by using the GSAS fitted parameters for each sample. As assumed in intergrowth model, the layered structure is piled up alternatively by pure $\mathrm{Li}_{2} \mathrm{MnO}_{3}$ phase and $\mathrm{LiCoO}_{2}$ phase. So, parameters adopted for the two end members (\#6586 and \#2778) in the intergrowth model use GSAS fitting results implemented in their pure phase cases respectively. For each composition except for \#6586, total 11 database curves (stacking fault probability (SFP) from 0\% to 100\%) are generated with $10 \%$ interval.

Table C2.1 Numbered sample and corresponding $\mathrm{LiCoO}_{2}$ composition.

\begin{tabular}{|c|c|c|c|c|c|c|c|}
\hline Sample \# & $\mathbf{6 5 8 6}$ & $\mathbf{2 4 1 7}$ & $\mathbf{2 4 1 8}$ & $\mathbf{2 4 1 3}$ & $\mathbf{2 4 1 9}$ & $\mathbf{2 4 2 0}$ & $\mathbf{2 7 7 8}$ \\
\hline Composition $\boldsymbol{x}$ & 1.0 & 0.9 & 0.7 & 0.5 & 0.3 & 0.1 & 0.0 \\
\hline
\end{tabular}

As an example for obtaining our requisite curves as shown in Fig. C1.1 in previous section (Appendix C1), only similar $R_{p}$ value curves (blue dotted lines) need to be constructed for each sample in Table C2.1 by repeatedly applying simulated annealing algorithm. The actual annealing parameters we used during such fitting procedure are summarized in table C2.2. Due to the high complexity nature of such non-linear fitting problem, total 100 repeated simulated annealing procedures are performed for each SFP database to increase their statistic. The smallest $R_{p}$ value is chosen among the 100 repeated cases as the final measurement of deviation between current SFP database and experiment. 
Such procedure is applied to all other composition databases to similarly obtain the statistically minimal $R_{p}$ values for each SFP curve and solid solutioncounterparts.

Table C2.2 Simulated annealing parameters for all single databases.

\begin{tabular}{|c|c|c|c|c|}
\hline $\begin{array}{l}\text { Temperature } \\
\text { start }\end{array}$ & $\begin{array}{c}\text { Temperature } \\
\text { end }\end{array}$ & $\begin{array}{c}\text { Temp. } \\
\text { descending } \\
\text { rate }\end{array}$ & $\begin{array}{c}\text { Step scale } \\
\text { factor }\end{array}$ & $\begin{array}{c}\text { Markov } \\
\text { chain }\end{array}$ \\
\hline 100 & $1 \mathrm{e}-5$ & 0.95 & 1.0 & 1000 \\
\hline
\end{tabular}

To further improve fitting and quantitatively obtain distribution of SFP for each composition, all the SFP databases for each composition are used to do the weighted second stage fitting with simulated annealing. The normalized weight is assigned to each SPF curve. Second stage fitting is for the purpose to search weight percentage of SFP distribution resulting even better $R_{p}$ value. It is worth to mention that \#2419, \#2420 and \#2778 use the annealing parameters the same as Table C2.2 for second stage search; however, \#2417 and \#2418 adopt annealing parameters from Table C2.3. Such difference with separate choice for annealing parameters has physical meaning underneath. For \#2417 and \#2418, $R_{p}$ value for each SFP databases are very close, thus, second stage search should start with relatively low "temperature" to freeze in comparable comfortable position instead of with high "temperature" to get "lost" in amorphous state resulting in an unexpected larger value of $R_{p}$.

Table C2.3 Simulated annealing parameters for 2417 and 2418 for second stage search.

\begin{tabular}{|c|c|c|c|c|}
\hline $\begin{array}{l}\text { Temperature } \\
\text { start }\end{array}$ & $\begin{array}{c}\text { Temperature } \\
\text { end }\end{array}$ & $\begin{array}{c}\text { Temp. } \\
\text { descending } \\
\text { rate }\end{array}$ & $\begin{array}{c}\text { Step scale } \\
\text { factor }\end{array}$ & $\begin{array}{c}\text { Markov } \\
\text { chain }\end{array}$ \\
\hline $\mathbf{0 . 0 0 1}$ & $1 \mathrm{e}-6$ & 0.95 & 0.01 & 1000 \\
\hline
\end{tabular}




\section{Appendix D: Copyright Permission of Texts and Figures in Chapter 3 and Copyright Permission of Texts in Appendix A}

Chapter 3, Appendix A and part of section 2.1 were originally published in "Control of domain configurations and sizes in crystallographically engineered ferroelectric single crystals: Phase field modeling”, Appl. Phys. Lett., AIP Publishing LLC, 2010, 97, 1629011-3 and "Correlated nucleation and self-accommodating kinetic pathway of ferroelectric phase transformation”, J. Appl. Phys., AIP Publishing LLC, 2012, 111, 024105-1-13. Copies of the granted permissions for reproduction are shown below.

\section{AIP PUBLISHING LLC LICENSE TERMS AND CONDITIONS}

\begin{tabular}{|c|c|}
\hline \multicolumn{2}{|c|}{$\begin{array}{l}\text { All payments must be made in full to CCC. For payment instructions, please see } \\
\text { information listed at the bottom of this form. }\end{array}$} \\
\hline License Number & 3607250413762 \\
\hline Order Date & Apr 13, 2015 \\
\hline Publisher & AIP Publishing LLC \\
\hline Publication & Applied Physics Letters \\
\hline Article Title & $\begin{array}{l}\text { Control of domain configurations and sizes in crystallographically } \\
\text { engineered ferroelectric single crystals: Phase field modeling }\end{array}$ \\
\hline Author & Wei-Feng Rao,Ke-Wei Xiao,Tian-Le Cheng, et al. \\
\hline Online Publication Date & Oct 18,2010 \\
\hline Volume number & 97 \\
\hline Issue number & 16 \\
\hline Type of Use & Thesis/Dissertation \\
\hline Requestor type & Author (original article) \\
\hline Format & Print and electronic \\
\hline Portion & Excerpt (> 800 words) \\
\hline Will you be translating? & No \\
\hline $\begin{array}{l}\text { Title of your thesis / } \\
\text { dissertation }\end{array}$ & $\begin{array}{l}\text { Modeling and Simulation of Microstructures, Mechanisms, and } \\
\text { Diffraction Effects in Energy Materials: Ferroelectrics and Lithium } \\
\text { Ion Battery Cathode Materials }\end{array}$ \\
\hline Expected completion date & May 2015 \\
\hline $\begin{array}{l}\text { Estimated size (number of } \\
\text { pages) }\end{array}$ & 200 \\
\hline Total & 0.00 USD \\
\hline
\end{tabular}




\section{AIP PUBLISHING LLC LICENSE TERMS AND CONDITIONS}

Apr 13, 2015

\begin{tabular}{|c|c|}
\hline \multicolumn{2}{|c|}{$\begin{array}{l}\text { All payments must be made in full to CCC. For payment instructions, please see } \\
\text { information listed at the bottom of this form. }\end{array}$} \\
\hline \multicolumn{2}{|r|}{3607250534039} \\
\hline \multicolumn{2}{|r|}{ Apr 13, 2015} \\
\hline \multicolumn{2}{|r|}{ AIP Publishing LLC } \\
\hline \multicolumn{2}{|r|}{ Applied Physics Letters } \\
\hline Article Title & $\begin{array}{l}\text { Control of domain configurations and sizes in crystallographically } \\
\text { engineered ferroelectric single crystals: Phase field modeling }\end{array}$ \\
\hline Author & Wei-Feng Rao,Ke-Wei Xiao,Tian-Le Cheng, et al. \\
\hline Online Publication Date & Oct 18,2010 \\
\hline Volume number & 97 \\
\hline Issue number & 16 \\
\hline Type of Use & Thesis/Dissertation \\
\hline Requestor type & Author (original article) \\
\hline Format & Print and electronic \\
\hline Portion & Figure/Table \\
\hline Number of figures/tables & 3 \\
\hline $\begin{array}{l}\text { Title of your thesis / } \\
\text { dissertation }\end{array}$ & $\begin{array}{l}\text { Modeling and Simulation of Microstructures, Mechanisms, and } \\
\text { Diffraction Effects in Energy Materials: Ferroelectrics and Lithium } \\
\text { Ion Battery Cathode Materials }\end{array}$ \\
\hline Expected completion date & May 2015 \\
\hline $\begin{array}{l}\text { Estimated size (number of } \\
\text { pages) }\end{array}$ & 200 \\
\hline Total & 0.00 USD \\
\hline
\end{tabular}




\section{AIP PUBLISHING LLC LICENSE TERMS AND CONDITIONS}

Apr 13, 2015

\begin{tabular}{|c|c|}
\hline License Number & 3607230272405 \\
\hline Order Date & Apr 13, 2015 \\
\hline Publisher & AIP Publishing LLC \\
\hline Publication & Journal of Applied Physics \\
\hline Article Title & $\begin{array}{l}\text { Correlated nucleation and self-accommodating kinetic pathway of } \\
\text { ferroelectric phase transformation }\end{array}$ \\
\hline Author & Jie E. Zhou,Tian-Le Cheng,Yu U. Wang \\
\hline Online Publication Date & Jan 25, 2012 \\
\hline Volume number & 111 \\
\hline Issue number & 2 \\
\hline Type of Use & Thesis/Dissertation \\
\hline Requestor type & Author (original article) \\
\hline Format & Print and electronic \\
\hline Portion & Excerpt (> 800 words) \\
\hline Will you be translating? & No \\
\hline $\begin{array}{l}\text { Title of your thesis / } \\
\text { dissertation }\end{array}$ & $\begin{array}{l}\text { Modeling and Simulation of Microstructures, Mechanisms, and } \\
\text { Diffraction Effects in Energy Materials: Ferroelectrics and Lithium } \\
\text { Ion Battery Cathode Materials }\end{array}$ \\
\hline Expected completion date & May 2015 \\
\hline $\begin{array}{l}\text { Estimated size (number of } \\
\text { pages) }\end{array}$ & 200 \\
\hline Total & 0.00 USD \\
\hline
\end{tabular}




\section{AIP PUBLISHING LLC LICENSE TERMS AND CONDITIONS}

Apr 13, 2015

\begin{tabular}{|c|c|}
\hline \multicolumn{2}{|c|}{$\begin{array}{l}\text { All payments must be made in full to CCC. For payment instructions, please see } \\
\text { information listed at the bottom of this form. }\end{array}$} \\
\hline License Number & 3607250726110 \\
\hline Order Date & Apr 13, 2015 \\
\hline Publisher & AIP Publishing LLC \\
\hline Publication & Journal of Applied Physics \\
\hline Article Title & $\begin{array}{l}\text { Correlated nucleation and self-accommodating kinetic pathway of } \\
\text { ferroelectric phase transformation }\end{array}$ \\
\hline Author & Jie E. Zhou,Tian-Le Cheng,Yu U. Wang \\
\hline Online Publication Date & Jan 25, 2012 \\
\hline Volume number & 111 \\
\hline Issue number & 2 \\
\hline Type of Use & Thesis/Dissertation \\
\hline Requestor type & Author (original article) \\
\hline Format & Print and electronic \\
\hline Portion & Figure/Table \\
\hline Number of figures/tables & 8 \\
\hline $\begin{array}{l}\text { Title of your thesis / } \\
\text { dissertation }\end{array}$ & $\begin{array}{l}\text { Modeling and Simulation of Microstructures, Mechanisms, and } \\
\text { Diffraction Effects in Energy Materials: Ferroelectrics and Lithium } \\
\text { Ion Battery Cathode Materials }\end{array}$ \\
\hline Expected completion date & May 2015 \\
\hline $\begin{array}{l}\text { Estimated size (number of } \\
\text { pages) }\end{array}$ & 200 \\
\hline Total & 0.00 USD \\
\hline
\end{tabular}




\section{Appendix E: Copyright Permission of Texts and Figures in Chapter 4}

Part of texts in Chapter 4 and part of sections 2.2 and 2.4 of this dissertation are

extracted from "Computational Study of Texture Development during Templated Grain Growth", originally published in EPD Congress 2014, John Wiley \& Sons, Inc., pages 299307. A copy of the granted permission for reproduction is shown below.

JOHN WILEY AND SONS LICENSE

TERMS AND CONDITIONS

Apr 13, 2015

\begin{tabular}{|c|c|}
\hline License Number & 3607250165203 \\
\hline License date & Apr 13, 2015 \\
\hline Licensed Content Publisher & John Wiley and Sons \\
\hline Licensed Content Publication & Wiley oBooks \\
\hline Licensed Content Title & $\begin{array}{l}\text { Computational Study of Texture Development During Templated } \\
\text { Grain Growth }\end{array}$ \\
\hline Licensed Content Author & Jie E. Zhou,Yu U. Wang \\
\hline Licensed Content Date & Jan 17, 2014 \\
\hline Pages & 9 \\
\hline Type of use & Dissertation/Thesis \\
\hline Requestor type & Author of this Wiley chapter \\
\hline Format & Print and electronic \\
\hline Portion & Text extract \\
\hline Number of Pages & 1 \\
\hline Will you be translating? & No \\
\hline $\begin{array}{l}\text { Title of your thesis / } \\
\text { dissertation }\end{array}$ & $\begin{array}{l}\text { Modeling and Simulation of Microstructures, Mechanisms, and } \\
\text { Diffraction Effects in Energy Materials: Ferroelectrics and Lithium } \\
\text { Ion Battery Cathode Materials }\end{array}$ \\
\hline Expected completion date & May 2015 \\
\hline $\begin{array}{l}\text { Expected size (number of } \\
\text { pages) }\end{array}$ & 200 \\
\hline Total & 0.00 USD \\
\hline
\end{tabular}


Portions of Figs. 4.1, 4.2, 4.4, 4.12 in Chapter 4 of this dissertation are extracted from “Computational Study of Texture Development during Templated Grain Growth", originally published in EPD Congress 2014, John Wiley \& Sons, Inc., pages 299-307. A copy of the granted permission for reproduction is shown below.

\section{JOHN WILEY AND SONS LICENSE TERMS AND CONDITIONS}

Jun 25,2015

This Agreement between Jie Zhou ("You") and John Wiley and Sons ("John Wiley and Sons") consists of your license details and the terms and conditions provided by John Wiley and Sons and Copyright Clearance Center.

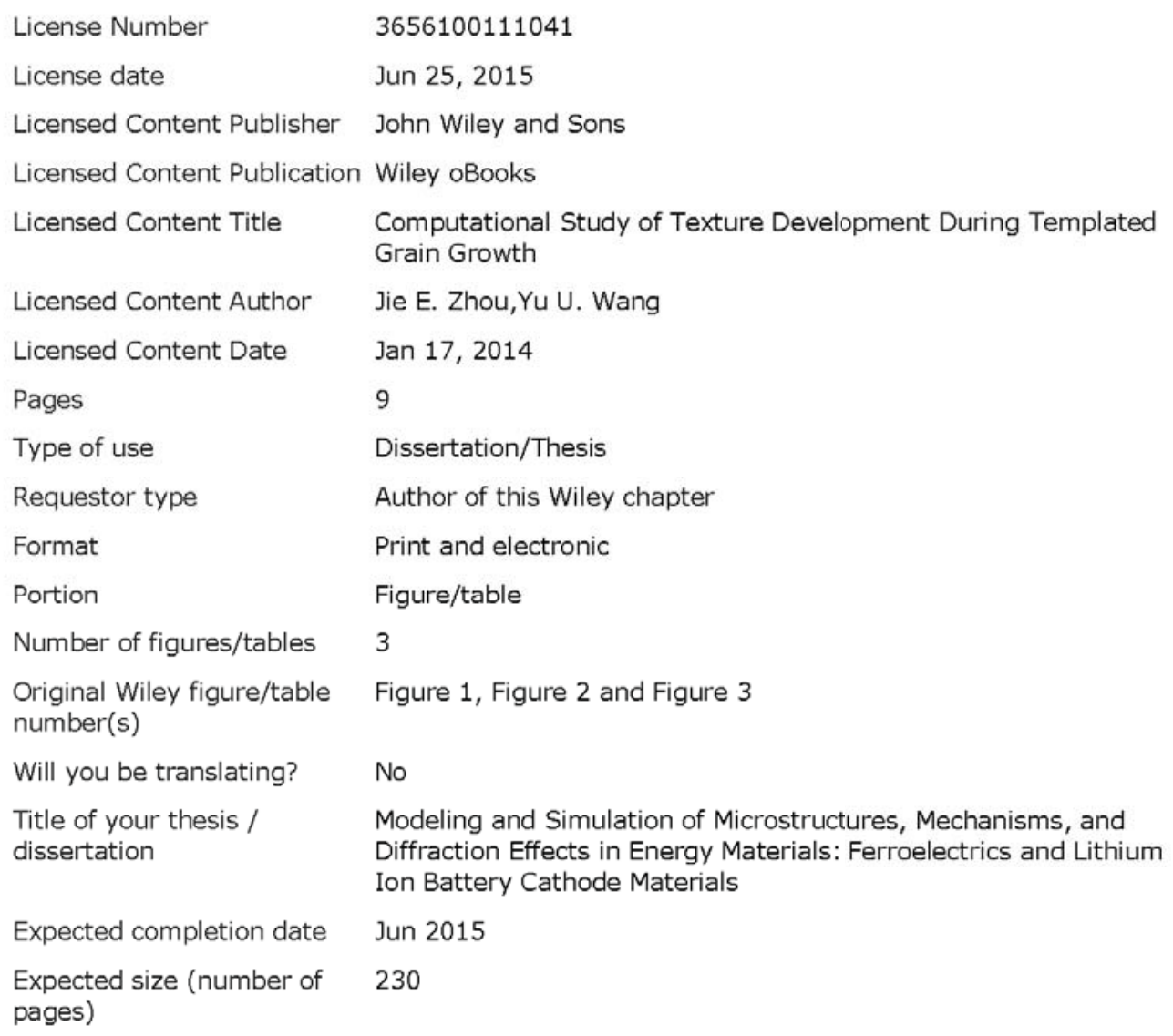




\section{Appendix F: Copyright Permission of Figures in Chapter 1}

Figure 1.1(b) in Chapter 1 reuses Fig. 1 originally published in "Dielectric behavior

of single crystals near the $(1-x) \quad \mathrm{Pb}\left(\mathrm{Mg}_{1 / 3} \mathrm{Nb}_{2 / 3}\right) \mathrm{O}_{3}-(x) \mathrm{PbTiO}_{3}$ morphotropic phase boundary", Ferroelectrics Letters Section, 12 (1990) 63-69.. Following is the request copyright permission.

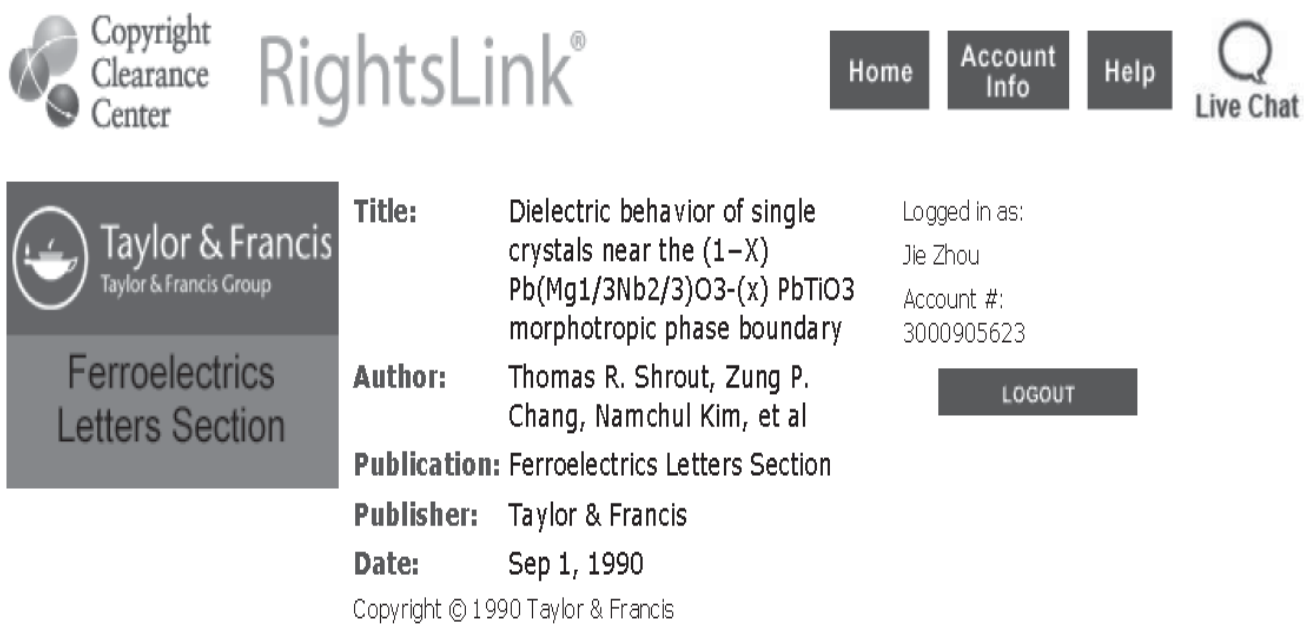

Thesis/Dissertation Reuse Request

Taylor \& Francis is pleased to offer reuses of its content for a thesis or dissertation free of charge contingent on resubmission of permission request if work is published. 
Figure 1.1(c) in Chapter 1 reuses Fig. 5 originally published in "Phase transitions in the $\mathrm{Pb}\left(\mathrm{Zn}_{1 / 3} \mathrm{Nb}_{2 / 3}\right) \mathrm{O}_{3}-\mathrm{PbTiO}_{3}$ system", Ferroelectrics, 37 (1981) 579-582. Following is the request copyright permission.

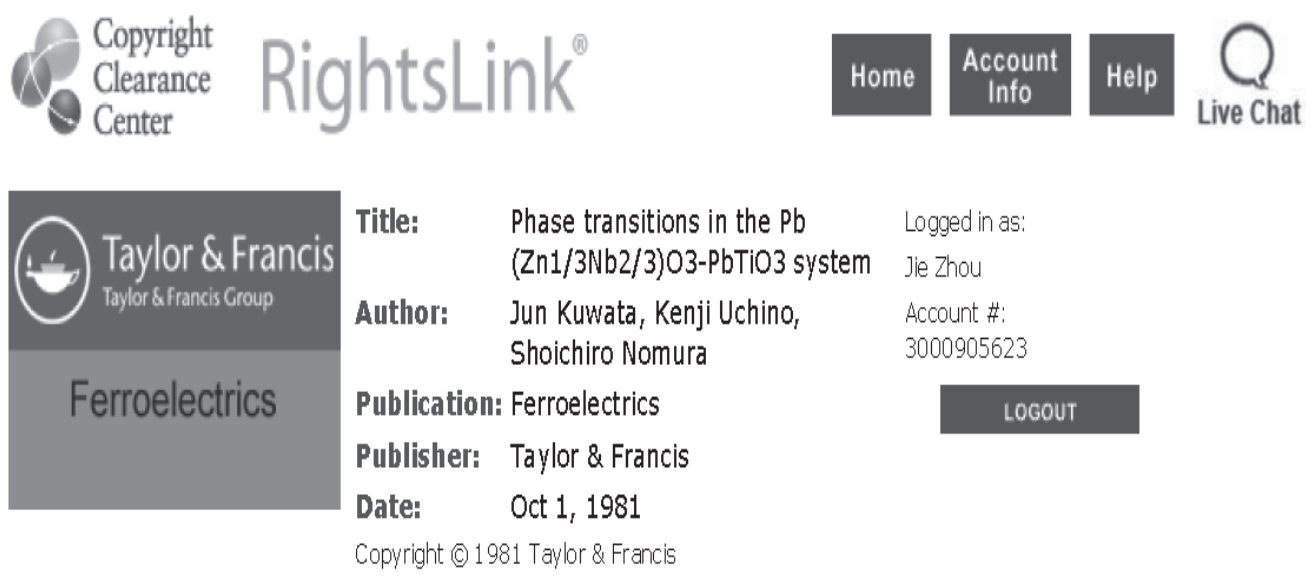

Thesis/Dissertation Reuse Request

Taylor \& Francis is pleased to offer reuses of its content for a thesis or dissertation free of charge contingent on resubmission of permission request if work is published. 


\section{Appendix G: Permission for Use of Figure 4.10 in Chapter 4}

Permission for the use of Figure 4.10 in Chapter 4 is presented as following email communications:
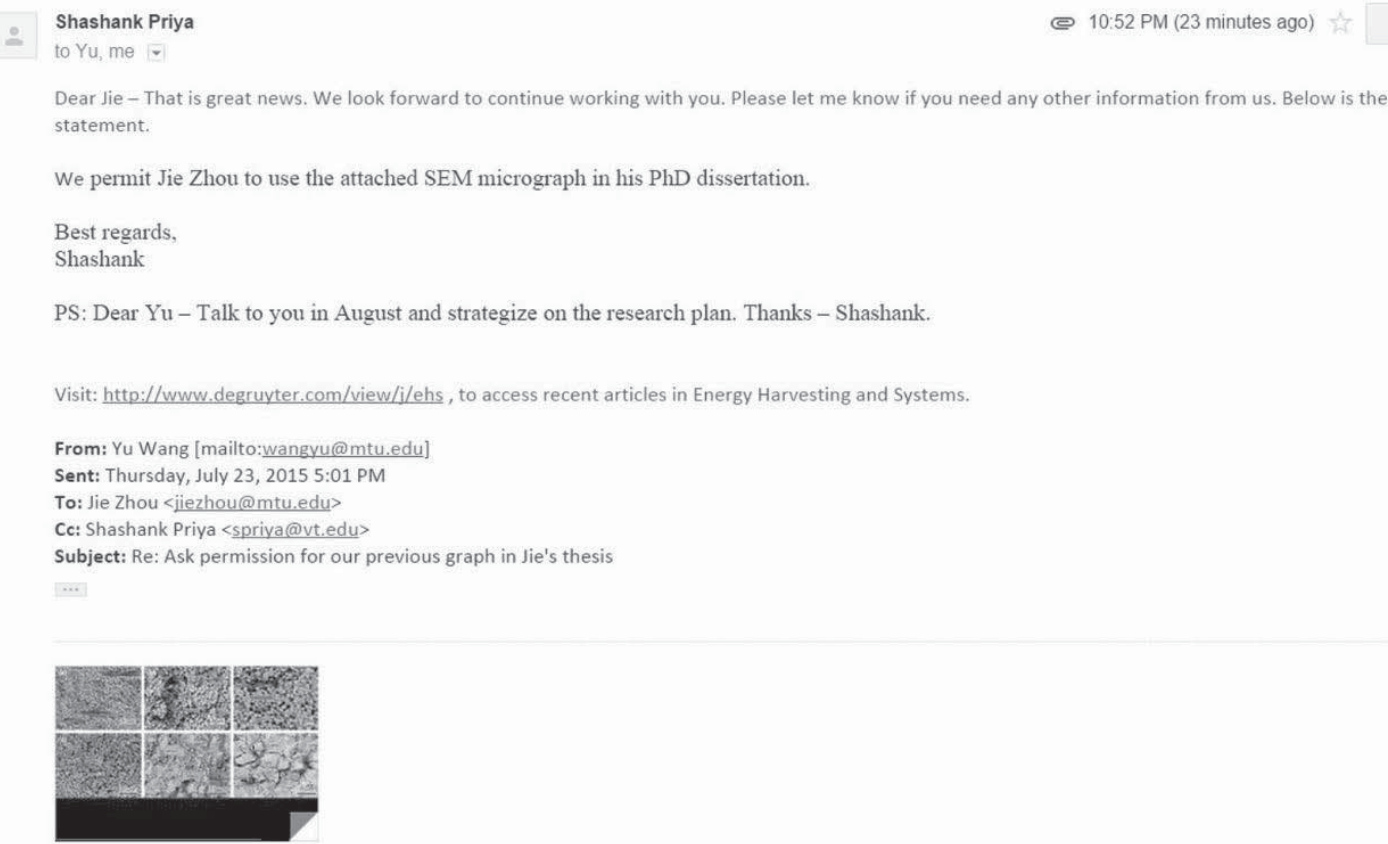